\title{
Analytische und numerische Untersuchungen bei inversen Transmissionsproblemen zur zeitharmonischen Wellengleichung
}

\author{
Dissertation \\ zur Erlangung des Doktorgrades \\ der Mathematisch-Naturwissenschaftlichen Fachbereiche \\ der Georg-August-Universität zu Göttingen
}

vorgelegt von

Christoph Schormann

aus Stuttgart

Göttingen 2000 
D 7

Referent: Prof. Dr. R. Kreß

Korreferent: Prof. Dr. G. Lube

Tag der mündlichen Prüfung: 20.6.2000 


\section{Inhaltsverzeichnis}

1 Einleitung 5

1.1 Transmissionsproblem zur Helmholtz-Gleichung . . . . . . . . . . . 5

1.2 Inhalt der Arbeit . . . . . . . . . . . . . . . . . . 8

2 Notation und etwas Differentialgeometrie 13

3 Potentialoperatoren 23

3.1 Einfach- und Doppelschichtpotentialoperator . . . . . . . . . . . . 23

3.2 Volumenpotentialoperator . . . . . . . . . . . . . . . . 33

4 Randwertprobleme $\quad 39$

4.1 Robinproblem . . . . . . . . . . . . . . . . . . . 40

4.2 Dirichletproblem . . . . . . . . . . . . . . . . . . . . . 42

4.3 Neumannproblem . . . . . . . . . . . . . . . . . . . 42

5 Transmissionsproblem $\quad \mathbf{4 5}$

5.1 Eindeutigkeit . . . . . . . . . . . . . . . . . . . . . . . 47

5.2 Potentialansatz . . . . . . . . . . . . . . . . 50

5.3 Greenscher Ansatz . . . . . . . . . . . . . . . . . . . 53

5.4 Ansatz von Kleinman und Martin . . . . . . . . . . . . . . . . . 56

5.5 Inhomogenes Transmissionsproblem . . . . . . . . . . . . . 59

6 Analytische Abhängigkeit von den Problemdaten $\quad \mathbf{6 1}$

6.1 Analytische Abbildungen . . . . . . . . . . . . . . . . 61

6.2 Ableitung nach dem Rand . . . . . . . . . . . . . . . . . . 62

6.3 Ableitung nach der Wellenzahl . . . . . . . . . . . . . . . . . . . . . 72

6.4 Ableitung nach der Transmissionskonstanten $\rho \ldots \ldots$. . . . . . . . . 74

6.5 Vergleich mit anderen Resultaten . . . . . . . . . . . . . . . . 75

7 Numerische Ergebnisse $\quad 77$

7.1 Numerische Lösung des direkten Problems . . . . . . . . . . . . . . . . 77

7.2 Berechnung der Fréchet-Ableitungen . . . . . . . . . . . . . . . 96

7.3 Inverse Transmissionsprobleme . . . . . . . . . . . . . . . . . . . . . . . 99

7.4 Vergleich mit anderen Resultaten und Fazit . . . . . . . . . . . . . . . 108

$\begin{array}{ll}\text { Literatur } & 113\end{array}$ 



\section{Einleitung}

\subsection{Transmissionsproblem zur Helmholtz-Gleichung}

Transmissionsprobleme zur skalaren Helmholtz-Gleichung treten als mathematische Modelle in verschiedenen Bereichen der Physik auf. Als Beispiel betrachten wir die Streuung von elektromagnetischen Wellen.

Gegeben sei hierzu ein homogenes, isotropes Medium im $\mathbb{R}^{3}$ mit Dielektrizitätskonstante $\epsilon$, Permeabilitätskonstante $\mu$ und elektrischer Leitfähigkeit $\sigma$, wobei stets $\epsilon, \mu, \sigma>0$ gelte. Eine elektromagnetische Welle läßt sich nun beschreiben durch ein elektrisches Feld $\mathfrak{E}$ und ein magnetisches Feld $\mathfrak{H}$, die die Maxwell-Gleichungen

$$
\operatorname{rot} \mathfrak{E}+\mu \frac{\partial \mathfrak{H}}{\partial t}=0, \quad \operatorname{rot} \mathfrak{H}-\epsilon \frac{\partial \mathfrak{E}}{\partial t}=\sigma \mathfrak{E},
$$

erfüllen. Bei zeitharmonische Wellen der Form

$$
\mathfrak{E}(x, t)=\operatorname{Re}\left\{\left(\epsilon+\frac{i \sigma}{\omega}\right)^{-1 / 2} E(x) e^{-i \omega t}\right\}, \quad \mathfrak{H}(x, t)=\operatorname{Re}\left\{\mu^{-1 / 2} H(x) e^{-i \omega t}\right\}, \quad \omega>0
$$

erfüllen die ortsabhängigen, komplexwertigen Funktionen $E$ und $H$ folgende (reduzierte) Maxwell-Gleichungen:

$$
\operatorname{rot} E-i \kappa H=0, \quad \operatorname{rot} H+i \kappa E=0 .
$$

Hierbei genügt die Wellenzahl $\kappa$ der Gleichung $\kappa^{2}=\lambda \mu \omega^{2}, \lambda:=\epsilon+i(\sigma / \omega)$, wobei das Vorzeichen von $\lambda^{1 / 2}$ so gewählt ist, daß die Ungleichungen $\operatorname{Im} \lambda^{1 / 2} \geq 0$ und $\operatorname{Re} \lambda^{1 / 2} \geq 0$ gelten.

Nun betrachten wir eine Unterteilung des $\mathbb{R}^{3}$ in bzgl. der dritten Koordinatenachse zylinderförmige Gebiete $\left\{\Omega_{l}\right\}_{l=0}^{L}$. Der Normalenvektor $\nu$ bzgl. eines Randes $\partial \Omega_{l}$ hat also die Form $\nu=\left(\nu_{1}, \nu_{2}, 0\right)$. Das Gebiet $\Omega_{0}$ sei bzgl. der ersten beiden Koordinaten unbeschränkt, die anderen beschränkt. Nun betrachten wir in $\Omega_{0}$ ein einfallendes elektromagnetisches Feld $\left(E_{i}, H_{i}\right)$, das in $\Omega_{0}$ ein gestreutes Feld $\left(E_{s}, H_{s}\right)$ und in $\Omega_{l}$ transmittierte Felder $\left(E_{l}, H_{l}\right), l=1, \ldots, L$ erzeugt. Sei $\left(E_{0}, H_{0}\right):=\left(E_{i}, H_{i}\right)+\left(E_{s}, H_{s}\right)$. Wir setzen voraus, daß alle Felder $\left(E_{l}, H_{l}\right), l=0, \ldots, L$ die Maxwell-Gleichungen mit Konstanten $\epsilon_{l}, \lambda_{l}, \kappa_{l}$ und $\mu_{l}$ erfüllen. Für zwei Gebiete $\Omega_{j}, \Omega_{l}$ mit $\partial \Omega_{j} \cap \partial \Omega_{l} \neq \emptyset$ gilt dann

$$
\nu \times \lambda_{j}^{-1 / 2} E_{j}=\nu \times \lambda_{l}^{-1 / 2} E_{l} \text { und } \nu \times \mu_{j}^{-1 / 2} H_{j}=\nu \times \mu_{l}^{-1 / 2} H_{l},
$$

da die Tangentialkomponenten der Felder $\left(\mathfrak{E}_{j}, \mathfrak{H}_{j}\right)$ und $\left(\mathfrak{E}_{l}, \mathfrak{H}_{l}\right)$ stetig sind (hierbei bezeichnet $a \times b$ das Kreuzprodukt im $\left.\mathbb{R}^{3}\right)$. 
Bei geeigneter Polarisierung des elektrischen Feldes ergibt sich nun ein Transmissionsproblem zur skalaren Helmholtz-Gleichung. Es gelte hierzu $E_{l}\left(x_{1}, x_{2}, x_{3}\right)=$ $\left(0,0, v_{l}\left(x_{1}, x_{2}\right)\right), l=0, \ldots, L$ und ebenso $E_{i}\left(x_{1}, x_{2}, x_{3}\right)=\left(0,0, v_{i}\left(x_{1}, x_{2}\right)\right)$. Die magnetischen Felder $H_{l}$ erfüllen dann die Gleichung

$$
H_{l}=\frac{1}{i \kappa_{l}} \operatorname{rot} E_{l}=\frac{1}{i \kappa_{l}}\left(\begin{array}{c}
\frac{\partial v_{l}}{\partial x_{2}} \\
-\frac{\partial v_{l}}{\partial x_{1}} \\
0
\end{array}\right) .
$$

Die Funktion $v_{l}$ ist hierbei eine Lösung der skalaren Helmholtz-Gleichung $\left(\Delta+\kappa_{l}^{2}\right) v_{l}=$ 0 . Da andererseits ein solchermaßen gewähltes Vektorfeld $E_{l}$ automatisch divergenzfrei ist, erfüllen $E_{l}$ und $H_{l}:=\frac{1}{i k} \operatorname{rot} E$ die Maxwell-Gleichungen, falls $v_{l}$ Lösung der skalaren Helmholtzgleichung ist. Wegen der Geometrie der Gebiete $\Omega_{l}$ ist es sinnvoll, die zweidimensionale Sommerfeldsche Ausstrahlungsbedingung zu verwenden, d.h. wir fordern von $v_{s}:=v_{0}-v_{i}$

$$
\frac{\partial v_{s}}{\partial r}-i \kappa_{0} v_{s}=\mathrm{o}\left(r^{-1 / 2}\right), \quad r:=|x| \rightarrow \infty
$$

gleichmäßig für alle Richtungen $\hat{x}:=x /|x|$.

Für Felder dieser speziellen Gestalt erhalten wir folgende Randbedingungen:

$$
\lambda_{j}^{-1 / 2} v_{j}=\lambda_{l}^{-1 / 2} v_{l}, \quad \frac{1}{\kappa_{j} \mu_{j}^{1 / 2}} \frac{\partial v_{j}}{\partial n}=\frac{1}{\kappa_{l} \mu_{l}^{1 / 2}} \frac{\partial v_{l}}{\partial n} .
$$

Diese Randbedingungen können durch die Normierung $u_{j}:=\lambda_{j}^{-1 / 2} v_{j}, j=0, \ldots, L$, noch etwas vereinfacht werden und ergeben sich dann zu

$$
u_{j}=u_{l}, \quad \frac{1}{\mu_{j}} \frac{\partial u_{j}}{\partial \nu}=\frac{1}{\mu_{l}} \frac{\partial u_{l}}{\partial \nu} .
$$

Für eine ausführlichere Darstellung verweisen wir auf [42]. Auch andere physikalische Vorgänge führen auf ein Transmissionsproblem zur Helmholtz-Gleichung. Exemplarisch verweisen wir nur noch auf die Streuung zeitharmonischer akustischer Wellen an durchstrahlbaren, homogenen Objekten.

Grundlage unserer Untersuchungen ist also folgendes

Problem 1.1. Gegeben sei eine Zerlegung des $\mathbb{R}^{d}, d=2,3$, in Gebiete $\Omega_{l}, l=0, \ldots, L$. Das Gebiet $\Omega_{0}$ sei unbeschränkt, die anderen Gebiete beschränkt. Weiterhin existieren Wellenzahlen $\kappa_{l}, l=0, \ldots, L$ und Übergangskonstanten $\rho_{l}, l=1, \ldots, L$.

Gesucht sind Funktionen $u_{s}$ in $\Omega_{0}$ und $u_{l}$ in $\Omega_{l}, l=1, \ldots, L$ mit

$$
\left(\Delta+\kappa_{l}^{2}\right) u_{l}=0, \quad l=0, \ldots, L .
$$

Hierbei sei $u_{0}:=u_{s}+u_{i}$ und $u_{i}(x):=\exp \left(i \kappa_{0} x \cdot v\right),|v|=1$. Für Gebiete $\Omega_{j}$ und $\Omega_{l}$ mit $\partial \Omega_{j} \cap \partial \Omega_{l} \neq \emptyset$ gelten die Randbedingungen

$$
u_{j}=u_{l}, \quad \rho_{l} \frac{\partial u_{j}}{\partial \nu}=\frac{\partial u_{l}}{\partial \nu} .
$$


Zudem erfülle $u_{s}$ die Sommerfeldsche Ausstrahlungsbedingung:

$$
\frac{\partial u_{s}}{\partial r}-i \kappa_{0} u_{s}=\mathrm{o}\left(r^{-(d-1) / 2}\right), \quad r:=|x| \rightarrow \infty,
$$

gleichmäßig für alle Richtungen $\hat{x}:=x /|x|$.

Genügt $u_{s}$ der Sommerfeldschen Ausstrahlungsbedingung, dann hat die Funktion folgendes asymptotische Verhalten:

$$
u_{s}(x)=\frac{e^{i \kappa_{0}|x|}}{|x|^{(d-1) / 2}}\left(u_{\infty}(\hat{x})+\mathrm{O}\left(\frac{1}{|x|}\right)\right), \quad|x| \rightarrow \infty .
$$

Die auf der Einheitskugel (bzw. dem Einheitskreis) definierte Funktion $u_{\infty}$ bezeichnen wir als das Fernfeld von $u_{s}$.

Neben dem eben skizzierten Problem ist auch die folgende Fragestellung von Interesse: Gegeben sei die Funktion $u_{i}$ und das (gemessene) Fernfeld der Funktion $u_{s}$. Zu bestimmen sind die Ränder $\partial \Omega_{l}$, die inneren Wellenzahlen $\kappa_{l}$ und die Übergangskonstanten $\rho_{l}, l=1, \ldots, L$. In Operatornotation enspricht dies dem Lösen einer Gleichung

$$
F\left(\left(\partial \Omega_{l}, \kappa_{l}, \rho_{l}\right)_{l=1}^{L}\right)=u_{\infty}
$$

Diese Fragestellung wird auch als inverses Problem bezeichnet, während die davor formulierte Aufgabe direktes Problem genannt wird. Wie im Vorwort von [32] beschrieben, werden dabei zwei Probleme zueinander invers genannt, falls die Formulierung des einen zumindest teilweise die Lösung des anderen Problems benötigt. Somit ist es zunächst willkürlich, welches Problem als direktes und welches als inverses bezeichnet wird. Häufig ist jedoch eine der beiden Aufgabenstellungen leichter zu behandeln, oder wurde bereits gründlicher untersucht. Diese wird dann normalerweise das direkte Problem genannt.

Dies trifft auch auf das hier betrachtete Transmissionsproblem zu. Während das direkte Problem zumindest für den Fall von nur zwei Gebieten $\Omega_{0}$ und $\Omega_{1}$ schon gründlich untersucht wurde (vgl. z.B. [40], [34] und [6]) und für die bestehenden numerischen Verfahren zur Approximation der Felder $u_{l}$ befriedigende Konvergenzaussagen existieren, sind beim inversen Transmissionsproblem noch eine Reihe von Fragen nicht, oder nur teilweise geklärt.

Der Grund hierfür ist ein wichtiger Unterschied zwischen den beiden Problemen. Nach Hadamard (vgl. [19]) wird ein Problem als korrekt gestellt bezeichnet, falls es eine eindeutige Lösung besitzt und diese Lösung stetig von den Daten abhängt. Falls zumindest eine der Bedingungen verletzt ist, nennt man das Problem inkorrekt gestellt.

Es zeigt sich, daß viele inverse Probleme in diesem Sinn inkorrekt gestellt sind. Dies trifft auch auf das hier betrachtete Transmissionsproblem zu. Der Grund hierfür ist die lokale Kompaktheit des Operators F (vgl. [57], Satz 5.9). Daher ist es von entscheidender Bedeutung, geeignete Regularisierungsverfahren zur numerischen Lösung des inversen Problems zu verwenden. 
Eine weitere Interpretation inverser Probleme wird in [23], Seite 11ff., gegeben. Häufig kann ein physikalisches Modell als ein Kausalzusammenhang aus Ursache und Wirkung betrachtet werden. Dies ist auch beim Transmissionsproblem der Fall. Die auftretenden Parameter des Problems (d.h. die Ränder der Gebiete, die Wellenzahlen und die Übergangskonstanten) können als Ursache, das Fernfeld als Wirkung des Transmissionsproblems verstanden werden. In diesem Sinn ist die Abbildung $F$ gerade die Ursache-Wirkung-Abbildung und Ziel des inversen Problems ist es, aus der Wirkung die Ursache zu rekonstruieren.

Zur Lösung von inversen Streuproblemen wurden in der Vergangenheit verschiedene Verfahren untersucht. Bei einer Klasse von Methoden (vgl. z.B. [7], Kapitel 5.4 und 5.5) wird das Problem in zwei Teilaufgaben zerlegt. Zunächst wird das gestreute Feld $u_{s}$ aus dem Fernfeld $u_{\infty}$ bestimmt. Dieses Problem ist linear und inkorrekt gestellt. Danach versucht man, mit Hilfe der Randbedingung von $u_{s}$ den Rand zu rekonstruieren. Hier ist das Problem nichtlinear, aber korrekt gestellt.

In neueren Untersuchungen von Colton und Kirsch (vgl. [5] und [33]) wurde der Fernfeldoperator $\mathcal{F}: L^{2}\left(S^{d-1}\right) \rightarrow L^{2}\left(S^{d-1}\right)$,

$$
\mathcal{F} g(\hat{x}):=\int_{S^{d-1}} u_{\infty}(\hat{x}, v) g(v) \mathrm{ds}(v)
$$

untersucht $\left(S^{d-1}:=\left\{v \in \mathbb{R}^{d}:|v|=1\right\}\right)$. Kirsch konnte in der Arbeit [33] einen exakten Zusammenhang zwischen dem singulären System von $\mathcal{F}$ und dem Gebiet $\Omega$ herstellen. Hieraus wurde ein numerisches Rekonstruktionsverfahren abgeleitet, das im Gegensatz zu anderen Verfahren keine a-priori Information über die betrachteten Gebiete benötigt. Allerdings benötigt man das Fernfeld $u_{\infty}(\cdot, v)$ für viele Einfallsrichtungen $v \in S^{d-1}$.

Als dritte Möglichkeit zur Rekonstruktion der Parameter wurden in den vergangenen Jahren intensiv iterative Verfahren studiert. Insbesondere für Newtonverfahren gibt es eine ganze Reihe von Untersuchungen, auf die wir im Kapitel 6 noch näher eingehen werden.

\subsection{Inhalt der Arbeit}

In dieser Arbeit untersuchen wir theoretische und numerische Aspekte bei einem regularisierten Newtonverfahren für das oben skizzierte inverse Transmissionsproblem. Ein wichtiger theoretischer Teil dieser Arbeit beschäftigt sich mit Existenz- und Eindeutigkeitsaussagen für Transmisssionsprobleme bei einer beliebig großen, endlichen Anzahl von Randkurven. Im Vergleich zu der Arbeit [47], die sich ebenfalls mit diesem Thema beschäftigt, geben wir explizite Algorithmen für verschiedene Lösungsansätze an.

In einem weiteren zentralen Teil der Arbeit weisen wir nach, daß die Funktion $u_{\infty}$ analytisch von den Rändern, den inneren Wellenzahlen und den Übergangsparametern abhängt. Den Beweis für die Differenzierbarkeit der Lösung eines Randwertproblems nach dem Rand, der auf einer Arbeit von Simon (vgl. [58]) beruht, wollen wir für das Dirichletproblem kurz skizzieren. 
Gegeben sei ein beschränktes $C^{m, \alpha}$-Gebiet $\Omega\left(m \in \mathbb{N}_{\geq 2}, 0<\alpha<1\right)$, das durch ein $C^{m, \alpha}$-Vektorfeld $\theta$ gestört wird. Sei $I_{\theta}:=I+\theta \cdot u[\theta] \in C^{m, \alpha}\left(\overline{\Omega_{\theta}}\right)$ sei die Lösung des Dirichletproblems zur Helmholtz-Gleichung in $\Omega_{\theta}:=I_{\theta}(\Omega)$ mit Randdaten $u[\theta]=-u_{i}$. Nun ist die Differenzierbarkeit der Abbildung $\theta \rightarrow u[\theta]$ nachzuweisen. Das Problem besteht hierbei darin, daß die Funktionen $u[\theta]$ unterschiedliche Definitionsbereiche haben. Ziel ist es daher eine geeignete Fortsetzung von $u[\theta]$ auf ganz $\mathbb{R}^{d}$ zu finden. Zunächst wird die Abbildung

$$
\tilde{u}[\theta]:=u[\theta] \circ I_{\theta} \in C^{m, \alpha}(\bar{\Omega})
$$

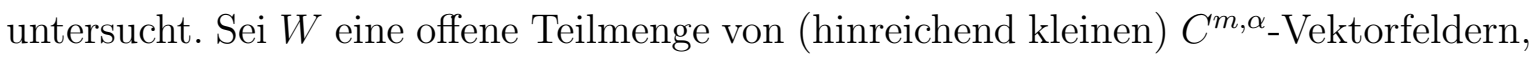
dann betrachtet man die Abbildung

$$
G: \begin{cases}W \times C^{m, \alpha}(\bar{\Omega}) & \rightarrow C^{m-2, \alpha}(\bar{\Omega}) \times C^{m, \alpha}(\partial \Omega) \\ (\theta, w) & \mapsto\left(\Delta_{\theta} w+\kappa^{2} w, w+u_{i} \circ I_{\theta}\right) .\end{cases}
$$

Hierbei ist $\Delta_{\theta}$ der transformierte Laplace-Operator, der durch $\Delta_{\theta}\left(v \circ I_{\theta}\right)=\Delta v \circ I_{\theta}$ für $v \in C^{2}\left(\overline{\Omega_{\theta}}\right)$ definiert ist. Weiterhin setzen wir voraus, daß $\kappa$ in $\Omega_{\theta}$ kein innerer Dirichlet-Eigenwert für $\theta \in W$ ist. Man kann leicht nachweisen, daß $G$ bzgl. beider Variablen Fréchet-differenzierbar ist. Zudem gilt

$$
G[\theta, \tilde{u}[\theta]]=\left(\Delta_{\theta} \tilde{u}[\theta]+\kappa^{2} \tilde{u}[\theta],\left(u[\theta]+u_{i}\right) \circ I_{\theta}\right)=\left(\left(\Delta u[\theta]+\kappa^{2} u[\theta]\right) \circ I_{\theta}, 0\right)=(0,0) .
$$

Die partielle Ableitung $G_{w}[0, u[0]] \in L\left(C^{m, \alpha}(\bar{\Omega}), C^{m-2, \alpha}(\bar{\Omega}) \times C^{m, \alpha}(\partial \Omega)\right)$ mit

$$
G_{w}[0, u[0]] p=\left(\Delta p+\kappa^{2} p, p\right)
$$

ist infolge der Lösbarkeit des Dirichlet-Problems für die inhomogene Laplace-Gleichung beschränkt invertierbar und somit folgt aus dem Satz über implizite Funktionen die Differenzierbarkeit der Abbildung $\theta \mapsto \tilde{u}[\theta]\left(W \rightarrow C^{m, \alpha}(\bar{\Omega})\right)$. Sei

$$
\left.E_{\Omega} \in L\left(C^{m, \alpha}(\bar{\Omega}), C^{m, \alpha}\left(\mathbb{R}^{d}\right)\right) \operatorname{mit}\left(E_{\Omega} v\right)\right|_{\bar{\Omega}}=v
$$

ein Fortsetzungsoperator und

$$
\tilde{U}[\theta]:=E_{\Omega} \tilde{u}[\theta]
$$

dann ist wegen der Linearität von $E_{\Omega}$ auch die Abbildung $\theta \mapsto \tilde{U}[\theta]\left(W \rightarrow C^{m, \alpha}\left(\mathbb{R}^{d}\right)\right)$ differenzierbar. Mit Hilfe der Taylorschen Formel und der Produktregel zeigt man die Differenzierbarkeit von

$$
U[\theta]:=\tilde{U}[\theta] \circ I_{\theta}^{-1}
$$

$\left(W \rightarrow C^{m-1, \alpha^{\prime}}\left(\mathbb{R}^{d}\right), 0<\alpha^{\prime}<\alpha\right)$ und die Darstellung

$$
U^{\prime}[0, h]=\tilde{U}^{\prime}[0, h]-\nabla U[0] \cdot h .
$$

Hier sei $a \cdot b\left(a, b \in \mathbb{R}^{d}\right)$ das Skalarprodukt im $\mathbb{R}^{d} . U[\theta]$ ist nun gerade die gesuchte Fortsetzung von $u[\theta]$, insbesondere gilt $\left.U[\theta]\right|_{\Omega_{\theta}}=\left.u[\theta]\right|_{\Omega_{\theta}}$. Sei $B \Subset \Omega$ eine Kugel und $h$ hinreichend klein, dann gilt $\left.U[h]\right|_{B}=\left.u[h]\right|_{B}$ und aus dem Greenschen Darstellungssatz, 
der für $u[h]$ in $B$ gilt, ergibt sich daher, daß $U^{\prime}[0, h]$ die Helmholtz-Gleichung in $B$ und damit in $\Omega$ löst. Offensichtlich ist die Restriktionsabbildung $\left.U\right|_{\partial \Omega}: W \rightarrow C^{m-1, \alpha^{\prime}}(\partial \Omega)$ ebenfalls differenzierbar und aus $\left.\nabla U[0]\right|_{\partial \Omega}=\left.\nabla u[0]\right|_{\partial \Omega}$ und $\left.\tilde{u}[\theta]\right|_{\partial \Omega}=-\left.u_{i} \circ I_{\theta}\right|_{\partial \Omega}$ (d.h. $\left.\left.\tilde{u}^{\prime}[0, h]\right|_{\partial \Omega}=-\nabla u_{i} \cdot h\right)$ folgt zusammen mit der Darstellungsformel (1.4) eine Gleichung für die Randwerte von $U^{\prime}[0, h]$ :

$$
\left.U^{\prime}[0, h]\right|_{\partial \Omega}=-\left.\nabla\left(u[0]+u_{i}\right) \cdot h\right|_{\partial \Omega}
$$

Diese Vorgehensweise hat gegenüber anderen Beweisen eine Reihe von Vorteilen. Zunächst werden keine Sobolev-Räume gebraucht, die Beweise sind dadurch erheblich elementarer. Weiterhin ist die Charakterisierung der Randwerte einfach möglich und der Beweis kann direkt auf die Untersuchung von höheren Ableitungen ausgedehnt werden.

Im Einzelnen ist die Arbeit folgendermaßen aufgebaut:

In Kapitel 2 haben wir die notwendigen Grundlagen bzgl. Notation und Art der betrachteten Gebiete zusammengestellt. Zentraler Teil dieses Kapitels sind Aussagen über Hölder-Räume. Wie sich bei dem eben skizzierten Beweis gezeigt hat, ermöglicht uns die Verwendung eines Fortsetzungsoperators (vgl. Definition 1.3), das Problem der unterschiedlichen Definitionsbereiche der Funktionen $u[\theta]$ zu lösen. Zudem erhalten wir dadurch auf einfache Weise die Randwerte von $u^{\prime}[0 ; h]$. In Kapitel 2 weisen wir deshalb die Existenz von Fortsetzungsoperatoren nach. Bei der Darstellung orientieren wir uns an Kapitel 6.9 aus [16].

Potentialoperatoren sind das Thema von Kapitel 3. Wir übertragen Untersuchungen von Kirsch (vgl. [29], [30]) zum Einfach- und Doppelschichtpotentialoperator im $\mathbb{R}^{3}$ auf den zweidimensionalen Fall. Betrachtet werden Abbildungseigenschaften in beliebigen Hölder-Räumen. Insbesondere beschäftigen wir uns mit Differenzen von Potentialen, die stärkere Glättungseigenschaften aufweisen. Diese Tatsache werden wir für den Existenznachweis beim Transmissionsproblem benutzen.

In der obigen Beweisskizze hatten wir gesehen, daß die Invertierbarkeit des Operators $G_{w}$ (vgl. (1.2)) gerade der eindeutigen Lösbarkeit eines Randwertproblems zur inhomogenen Helmholtzgleichung entspricht. Der zweite Teil dieses Kapitels beschäftigt sich daher mit dem Volumenpotentialoperator, den wir für solche Randwertprobleme zur inhomogenen Helmholtzgleichung verwenden werden. Auch hier beweisen wir Abbildungseigenschaften in beliebigen Hölder-Räumen. Dies ermöglicht uns die Behandlung von höheren Ableitungen der Funktion $u[\theta]$ bzw. des Operators $F$.

Die potentialtheoretischen Grundlagen versetzen uns nun in die Lage, Aussagen über Randwertprobleme zur inhomogenen Helmholtzgleichung in beliebigen HölderRäumen zu treffen. In Kapitel 4 untersuchen wir das Dirichlet-, das Neumann- und das Robinproblem.

Kapitel 5 behandelt das direkte Transmissionsproblem. Im Vergleich zu anderen Untersuchungen (z.B. [34], [40]), in denen die Situation bei einem zusammenhängenden Rand untersucht wurde, betrachten wir das Problem für sehr allgemeine Gebietskonstellationen, d.h. wir lassen eine beliebige große (endliche) Anzahl von Randkurven 
(bzw. Randflächen) zu. Zunächst weisen wir die Eindeutigkeit des Problems bei geeigneten Voraussetzungen an die auftretenden Konstanten nach. Der Existenzbeweis erfolgt konstruktiv durch Potentialoperatoren. Für $L$ Randkurven $(L \in \mathbb{N})$ erhält man ein eindeutig lösbares Gleichungssystem, mit einer $2 L \times 2 L$-Operatormatrix. Für das Aufstellen dieser Matrix geben wir explizite und einfach zu implementierende Algorithmen an, die wir numerisch auch erfolgreich getestet haben.

Nun sind wir im Besitz aller Hilfsmittel, um Aussagen bzgl. der Differenzierbarkeit von Lösungen der betrachteten Randwertprobleme nach den auftretenden Parametern (d.h. den Rändern der Gebiete, den inneren Wellenzahlen und den Übergangskonstanten) zu untersuchen. Dies ist das Thema von Kapitel 6. Die zugrunde liegende Beweisidee haben wir oben kurz dargestellt. Wie bereits erwähnt, führen wir den Beweis im Gegensatz zur Arbeit [58] in Hölder-Räumen durch. Es zeigt sich, daß die verschiedenen Randbedingungen einheitlich behandelt werden können. Weiterhin geben wir eine Charakterisierung der Randwerte beliebig hoher Ableitungen der gestreuten (bzw. transmittierten) Felder an. Die analytische Abhängigkeit dieser Funktionen bzgl. der inneren Wellenzahlen und der Übergangskonstanten kann mit der gleichen Beweisidee wie bei der Ableitung nach dem Rand nachgewiesen werden.

In Kapitel 7 wenden wir uns numerischen Fragen beim zweidimensionalen Transmissionsproblem zu. Zunächst geben wir eine Konvergenzanalyse für das direkte Problem an. Hierbei verwenden wir Projektionsverfahren mit trigonometrischen Polynomen. Die Aussagen werden uns als Grundlage für ein Verfahren zur Berechnung der Ableitung nach dem Rand dienen. Schließlich geben wir eine Reihe von numerischen Beispielen zum direkten Transmissionsproblem, zur Berechnung der Ableitungen und zur Rekonstruktion der auftretenden Parameter an. Meines Wissens nach werden in dieser Arbeit zum ersten Mal Transmissionsprobleme für mehrere Randkurven numerisch untersucht. Auch die Berechnung der Ableitungen nach den Wellenzahlen und Übergangskonstanten und ihre Verwendung zur Rekonstruktion dieser Parameter ist neu. Bei der Klasse der approximierbaren Objekte können wir vorhandene Ergebnisse erweitern, da wir auch nichtsternfömige Gebiete rekonstruieren und keine genauen a-priori Information über die Lage der Objekte benötigen. Insgesamt erweist sich das regularisierte Newton-Verfahren als ein vielversprechendes Rekonstruktionsverfahren beim inversen Transmissionsproblem zur zeitharmonischen Wellengleichung.

Für die fachliche Unterstützung und die Betreuung dieser Arbeit danke ich Herrn Prof. Dr. Rainer Kreß. Weiterhin möchte ich mich bei Herrn Prof. Dr. Gert Lube für die Übernahme des Korreferats und bei Herrn Priv. Doz. Dr. Peter Hähner für die zahlreichen Gespräche im Verlauf dieser Arbeit bedanken. Herrn Dipl.-Math. Andreas Vogt danke ich für das Korrekturlesen von Teilen der Arbeit. Bei der Deutschen Forschungsgemeinschaft möchte ich mich für die finanzielle Förderung bedanken. Für die Unterstützung gerade in der Endphase dieser Arbeit danke ich meinen Eltern und meinen Geschwistern. 
Göttingen, Mai 2000

Christoph Schormann 


\section{Notation und etwas Differentialgeometrie}

Sei $\Omega \subset \mathbb{R}^{d}, d=2,3$, ein Gebiet, das sowohl beschränkt, als auch unbeschränkt sein kann. Der Rand $\partial \Omega$ von $\Omega$ bestehe aus endlich vielen, beschränkten und regulären Zusammenhangskomponenten $\Gamma_{1}, \ldots, \Gamma_{L}, L \in \mathbb{N}$, die den $\mathbb{R}^{d}$ jeweils eindeutig in ein beschränktes Innengebiet $\Omega_{l, i}$ und ein unbeschränktes Außengebiet $\Omega_{l, a}$ zerlegen. Weiterhin sei $\Omega_{e}:=\mathbb{R}^{d} \backslash \bar{\Omega}, \nu$ der Normalenvektor und $\vartheta$ ein normierter Tangentialvektor in $\partial \Omega$. Für die Richtung des Normalenvektors verwenden wir folgende Konvention: Wird er bzgl. $\partial \Omega$ für ein beschränktes Gebiet $\Omega$ betrachtet, so zeige er in das Äußere dieses Gebietes, für ein unbeschränktes Gebiet zeige er in das Innere des Gebietes. Durch diese Vereinbarung behalten die Greenschen Sätze

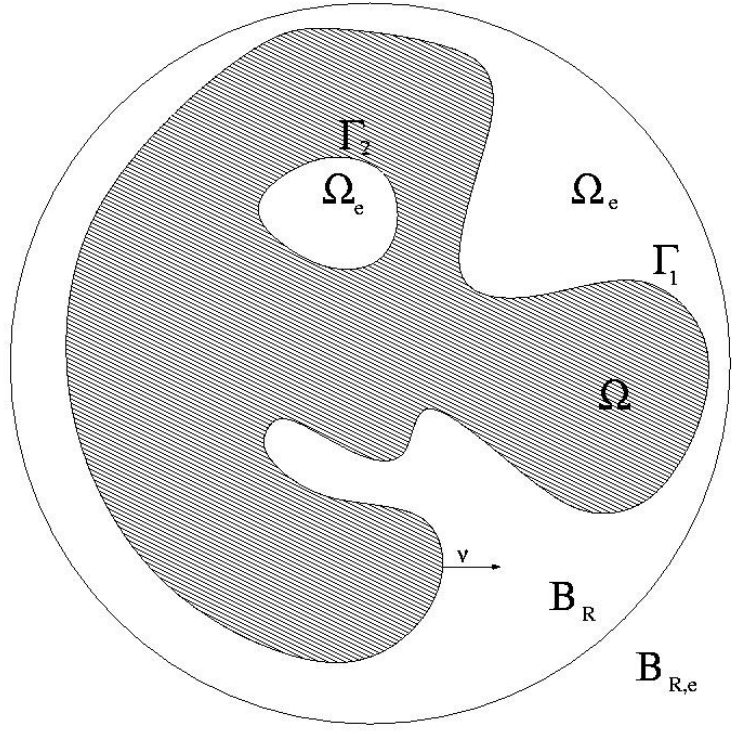

Abbildung 2.1: Beispielgeometrie und Darstellungssätze die aus der Situation bei nur einer Randkurve bekannten Vorzeichen (vgl. z.B. [7], Theorem 2.1 und 2.4) und auch die Sprungbeziehungen der Potentialtheorie haben die gewohnten Vorzeichen (vgl. [7], Theorem 3.1). Sei $R>0$ so groß, daß die Ränder $\Gamma_{1}, \ldots, \Gamma_{L}$ in $B_{R}:=B_{R}(0):=\left\{x \in \mathbb{R}^{d}:\|x\|<R\right\}$ enthalten sind. (vgl. die Abbildung 2.1). Wir definieren weiterhin $B_{R, e}:=\mathbb{R}^{d} \backslash \overline{B_{R}}$.

Für eine in $\Omega_{l, a}$ bzw. $\Omega_{l, i}$ definierte Funktion $u$ betrachtet man deren Grenzwert $u_{ \pm}(z), z \in \partial \Omega$, mit:

$$
u_{-}(z):=\lim _{\substack{x \rightarrow z \in \partial \Omega \\ x \in \Omega_{l, i}}} u(x), \quad u_{+}(z):=\lim _{\substack{x \rightarrow z \in \partial \Omega \\ x \in \Omega_{l, a}}} u(x)
$$

vorausgesetzt diese Werte existieren. Analog definiert man $\frac{\partial u_{\mp}}{\partial \nu}(z)$ :

$$
\frac{\partial u_{-}}{\partial \nu}(z):=\lim _{\substack{x \rightarrow z \in \partial \Omega \\ x \in \Omega_{l, i}}} \nabla u(x) \cdot \nu(z), \quad \frac{\partial u_{+}}{\partial \nu}(z):=\lim _{\substack{x \rightarrow z \in \partial \Omega \\ x \in \Omega_{l, a}}} \nabla u(x) \cdot \nu(z) .
$$

Für zwei Vektoren $x, y \in \mathbb{C}^{d}$ sei $x \cdot y:=\sum_{j=1}^{d} x_{j} y_{j} \in \mathbb{C}$ und $x \odot y:=\left(x_{i} y_{j}\right)_{i, j=1}^{d} \in$ 
$\mathbb{C}^{d \times d}$. Für das Produkt einer Matrix $M \in \mathbb{C}^{m \times d}$ und eines Vektors $v \in \mathbb{C}^{d}$ verwenden wir die Schreibweise $M \cdot v \in \mathbb{C}^{m}$.

Für zwei normierte Räume $X, Y$ bezeichnen wir mit $L(X, Y)$ den Raum der beschränkten, linearen Operatoren, die von $X$ nach $Y$ abbilden.

Für $x, y \in \mathbb{R}^{d}, x \neq y$, ist die Funktion

$$
\Phi(x, y):= \begin{cases}\frac{i}{4} H_{0}^{(1)}(\kappa|x-y|) & d=2, \\ \frac{\exp (i \kappa|x-y|)}{4 \pi|x-y|} & d=3\end{cases}
$$

die Grundlösung der Helmholtzgleichung im $\mathbb{R}^{d}$ zur Wellenzahl $\kappa \in\{z \in \mathbb{C} \backslash\{0\}$ : $\operatorname{Im}(z) \geq 0\}$ und

$$
\Phi_{0}(x, y):= \begin{cases}\frac{1}{2 \pi} \ln \frac{1}{|x-y|} & d=2, \\ \frac{1}{4 \pi|x-y|} & d=3,\end{cases}
$$

ist die Grundlösung der Laplacegleichung im $\mathbb{R}^{d}$.

Eine Funktion $u$ erfüllt die Sommerfeldsche Ausstrahlungsbedingung (SAB), falls

$$
\frac{\partial u}{\partial r}-i \kappa u=\mathrm{o}\left(r^{-\frac{d-1}{2}}\right), \quad r:=|x| \rightarrow \infty,
$$

gleichmäßig für alle Richtungen $x /|x| \in S^{d-1}$ gilt. Hierbei sei $S^{d-1}:=\left\{x \in \mathbb{R}^{d}:|x|=\right.$ $1\}$.

Für zwei in $\mathbb{R}^{d}$ offene Mengen $\Omega_{1}$ und $\Omega_{2}$ definieren wir folgende Inklusionsbeziehung:

$$
\Omega_{1} \Subset \Omega_{2} \text { falls } \overline{\Omega_{1}} \subset \Omega_{2} .
$$

Für $m \in \mathbb{N}_{0}$ sei $C^{m}(\Omega)$ der Raum der m-fach stetig differenzierbaren, komplexwertigen Funktionen auf $\Omega$. Für solche Funktionen sei

$$
\|u\|_{m}:=\max _{j=0, \ldots, m} \sup _{x \in \Omega}\left|\nabla^{j} u(x)\right| .
$$

Wie üblich ist hierbei

$$
\nabla^{j} u:=\left(\partial^{j} u / \partial x_{1}^{i_{1}} \ldots \partial x_{d}^{i_{d}}\right)_{\substack{i_{1}, \ldots, i_{d} \in \mathbb{N}_{0} \\ i_{1}+\ldots+i_{d}=j}}
$$

und die Norm der Multilinearform $\nabla^{j} u(x):\left(\mathbb{C}^{d}\right)^{j} \rightarrow \mathbb{C}$ ist erklärt durch

$$
\left|\nabla^{j} u(x)\right|:=\sup _{\substack{v_{1}, \ldots, v_{j} \in \mathbb{C}^{d} \\\left|v_{1}\right|=\ldots=\left|v_{j}\right|=1}}\left|\nabla^{j} u(x)\left(v_{1}, \ldots, v_{j}\right)\right| .
$$

Nun betrachten wir den Raum

$$
\begin{aligned}
C^{m}(\bar{\Omega}):= & \left\{u \in C^{m}(\Omega): \text { alle Ableitungen von } u \text { bis zur Ordnung } m \text { sind stetig auf } \bar{\Omega}\right. \\
& \text { fortsetzbar und } \left.\|u\|_{m}<\infty .\right\}
\end{aligned}
$$


Dieser Raum ist bekanntlich ein Banach-Raum.

Sei für den ganzen Abschnitt $\alpha \in] 0,1[$. Wir verwenden den Hölder-Raum

$$
C^{m, \alpha}(\bar{\Omega}):=\left\{u \in C^{m}(\bar{\Omega}): \exists c>0 \text { mit }\left|\nabla^{m} u(x)-\nabla^{m} u(y)\right| \leq c|x-y|^{\alpha} \text { für } x, y \in \bar{\Omega}\right\} .
$$

Auch dieser Raum ist ein Banach-Raum (für einen Beweis vgl. z.B. [6], Theorem 2.4.) mit der Norm

$$
\|u\|_{m, \alpha}:=\|u\|_{m}+\left|\nabla^{m} u\right|_{\alpha}
$$

wobei wir folgende Halbnorm benutzen:

$$
\left|\nabla^{m} u\right|_{\alpha}:=\sup _{\substack{x, y \in \bar{\Omega} \\ x \neq y}} \frac{\left|\nabla^{m} u(x)-\nabla^{m} u(y)\right|}{|x-y|^{\alpha}} .
$$

Ganz analog werden für offene Mengen $\Omega_{1}, \Omega_{2} \subset \mathbb{R}^{d}$ und vektorwertige Funktionen die Räume $C^{m, \alpha}\left(\overline{\Omega_{1}}, \overline{\Omega_{2}}\right)$ eingeführt. Zudem benötigen wir den Raum der lokal Hölderstetigen Funktionen auf $\Omega$ :

$$
C_{\mathrm{loc}}^{0, \alpha}(\Omega):=\left\{\varphi \in C(\Omega): \forall \Omega^{\prime} \Subset \Omega: \varphi \in C^{0, \alpha}\left(\overline{\Omega^{\prime}}\right)\right\} .
$$

Für $\Omega_{1}, \Omega_{2} \subset \mathbb{R}^{d}$ definieren wir

$$
\begin{gathered}
C^{0, \alpha, \beta}\left(\overline{\Omega_{1}} \times \overline{\Omega_{2}}\right):=\left\{w: \Omega_{1} \times \Omega_{2} \rightarrow \mathbb{C}: \exists C>0 \quad \forall x_{1}, x_{2} \in \Omega_{1}, y_{1}, y_{2} \in \Omega_{2}:\right. \\
\left.\left|w\left(x_{1}, y_{1}\right)-w\left(x_{2}, y_{2}\right)\right| \leq C\left(\left|x_{1}-x_{2}\right|^{\alpha}+\left|y_{1}-y_{2}\right|^{\beta}\right)\right\} .
\end{gathered}
$$

Neben diesen Funktionenräumen, die über Gebieten definiert sind, verwenden wir auch Funktionenräume über Rändern von Gebieten. (vgl. [29], Seite 5). Hierbei gehört der Rand $\Gamma$ eines Gebietes $\Omega$ zu der Klasse $C^{m, \alpha}$ (wir schreiben hierfür auch $\Gamma \in C^{m, \alpha}$ ), falls für alle $z \in \Gamma$ Umgebungen $V_{z}$ und bijektive Abbildungen $\psi_{z}: V_{z} \rightarrow B_{1} \subset \mathbb{R}^{d}$ existieren, die folgende Bedingungen erfüllen (vgl. die Abbildung 2.2):

1. $\psi_{z}\left(V_{z} \cap \Omega\right)=\left\{x=\left(x_{1}, \ldots, x_{d}\right) \in B_{1}: x_{d}>0\right\}$,

2. $\psi_{z}\left(V_{z} \cap \Gamma\right)=\left\{x \in B_{1}: x_{d}=0\right\}$,

3. $\psi_{z} \in C^{m, \alpha}\left(V_{z}\right), \psi_{z}^{-1} \in C^{m, \alpha}\left(B_{1}\right)$.

Mit dieser Definition können nun für $\Gamma \in C^{m}$ bzw. $\Gamma \in C^{m, \alpha}$ folgende Funktionenräume eingeführt werden:

$$
\begin{aligned}
C^{m}(\Gamma) & :=\left\{u: \Gamma \rightarrow \mathbb{C}:\left.u \circ \psi_{z}^{-1}\right|_{\mathbb{R}^{d-1}} \in C^{m}\left(\overline{B_{1}}\right) \text { mit } B_{1} \subset \mathbb{R}^{d-1} \text { und für alle } z \in \Gamma\right\}, \\
C^{m, \alpha}(\Gamma) & :=\left\{u \in C^{m}(\Gamma):\left.u \circ \psi_{z}^{-1}\right|_{\mathbb{R}^{d-1}} \in C^{m, \alpha}\left(\overline{B_{1}}\right) \text { für alle } z \in \Gamma\right\} .
\end{aligned}
$$

Auch diese Räume sind bekanntlich Banach-Räume, wobei durch verschiedene Parametrisierungen von $\Gamma$ äquivalente Normen entstehen. 
Neben dieser Definition wollen wir noch eine weitere, äquivalente Norm angeben, für die wir den Begriff des Oberflächengradienten benötigen (vgl. hierzu z.B. auch Kapitel 2.1 in [6]). Für einen $C^{2}$-Rand $\Gamma$ und eine offene Menge $U \subset \mathbb{R}^{d-1}$ betrachten wir eine zweimal stetig differenzierbare, bijektive Abbildung $x: U \rightarrow \Gamma$. Aufgrund der Injektivität von $x$ sind die Vektoren

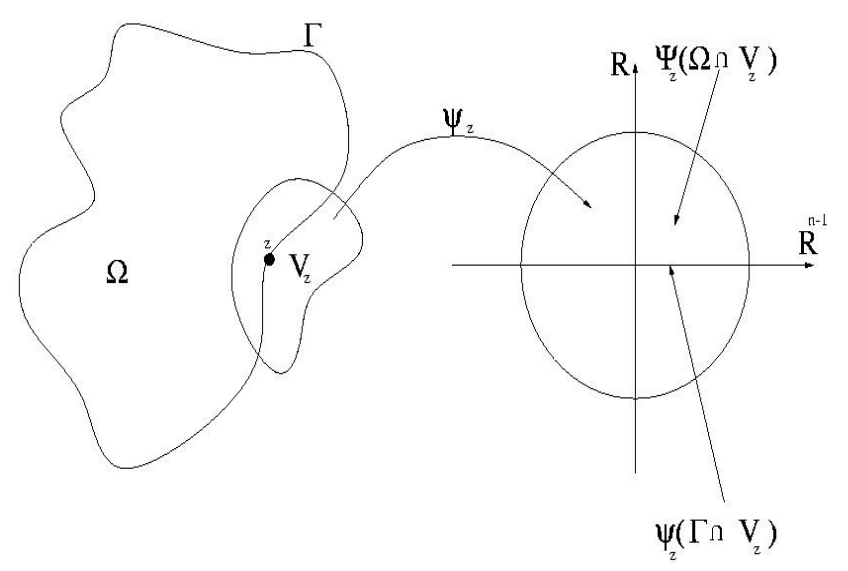

$$
x_{, j}:=\frac{\partial x}{\partial u_{j}}, \quad j \in\{1, \ldots, d-1\}
$$

Abbildung 2.2: Randparametrisierung

linear unabhängig und somit ist die symmetrische Matrix $\left(g_{j k}\right)$ mit

$$
g_{j k}:=x_{, j} \cdot x_{, k}, \quad j, k \in\{1, \ldots, d-1\}
$$

invertierbar. Bezeichnen wir mit $g^{j k}$ die Einträge der inversen Matrix, so ist der Oberflächengradient für eine Funktion $\varphi \in C^{1}(\Gamma)$ definiert als

$$
\operatorname{Grad} \varphi:=\sum_{j, k=1}^{d-1} g^{j k} \frac{\partial(\varphi \circ x)}{\partial u_{j}} x_{, k}
$$

Es läßt sich zeigen, daß Grad $\varphi$ unabhängig von der Parametrisierung $x$ ist. Im zweidimensionalen Fall vereinfacht sich dies zu $\operatorname{Grad} \varphi=\frac{1}{\left|x^{\prime}\right|^{2}}(\varphi \circ x)^{\prime} x^{\prime}$ für eine reguläre Parametrisierung $x$. Im $\mathbb{R}^{2}$ werden wir zusätzlich die Bezeichung

$$
\frac{\mathrm{d}}{\mathrm{ds}} \varphi(z(t)):=\frac{1}{\left|x^{\prime}(t)\right|}(\varphi \circ x)^{\prime}(t)=\operatorname{Grad} \varphi(z(t)) \cdot \vartheta(z(t))
$$

für einen Tangentialvektor $\vartheta(z(t))=\frac{x^{\prime}(t)}{\left|x^{\prime}(t)\right|}$ benutzen. Es gilt $\varphi \in C^{m, \alpha}(\Gamma)$ genau dann, wenn $\frac{\mathrm{d}}{\mathrm{ds}} \varphi \in C^{m-1, \alpha}(\Gamma)$. Falls $\varphi \in C^{1}(\bar{\Omega})$ folgt $\frac{\mathrm{d}}{\mathrm{ds}} \varphi=\nabla \varphi \cdot \vartheta$.

Sei $T_{x}$ der Tangentialraum in $x \in \Gamma$. Dann kann man induktiv die Multilinearform $\operatorname{Grad}^{j} \psi(x): T_{x}^{j} \rightarrow \mathbb{C}$ durch

$$
\operatorname{Grad}^{j} \psi(x)\left(\vartheta_{1}, \ldots, \vartheta_{j}\right):=\operatorname{Grad}^{j-1}\left(\vartheta_{j} \cdot \operatorname{Grad} \psi\right)(x)\left(\vartheta_{1}, \ldots, \vartheta_{j-1}\right)
$$

für $j \in \mathbb{N}$ definieren. Dies ermöglicht die Einführung einer äquivalenten Norm auf $C^{m, \alpha}(\Gamma)$ :

$$
\|\psi\|_{m, \alpha}:=\max _{j=0, \ldots, m} \max _{x \in \Gamma}\left|\operatorname{Grad}^{j} \psi(x)\right|+\left|\operatorname{Grad}^{m} \psi\right|_{\alpha, \Gamma} .
$$

Auch hier bezeichnen wir mit $|\cdot|$ die Norm der Multilinearformen. 
Im folgenden werden wir noch eine Aussage bzgl. der Fortsetzbarkeit von Funktionen, die auf Gebieten bzw. deren Rändern definiert sind, beweisen. Dies findet sich z.B. in Kapitel 6.9 aus [16]. Wir wiederholen der Vollständigkeit wegen den dort geführten Beweis. Zunächst beweisen wir, daß die Komposition von Hölder-stetigen Funktionen in konvexen Gebieten wieder Hölder-stetig ist.

Lemma 2.1. Seien $\Omega_{1}, \Omega_{2}$ konvexe $C^{m, \alpha}$-Gebiete, $f \in C^{m, \alpha}\left(\overline{\Omega_{2}}\right)$ und $g \in C^{m, \alpha}\left(\overline{\Omega_{1}}, \overline{\Omega_{2}}\right)$, dann folgt

$$
f \circ g \in C^{0, \alpha^{2}}\left(\overline{\Omega_{1}}\right) \text { für } m=0 \text { und } f \circ g \in C^{m, \alpha}\left(\overline{\Omega_{1}}\right) \text { für } m>0 \text {. }
$$

Weiterhin gelten die Abschätzungen

$$
\|f \circ g\|_{0, \alpha^{2}} \leq C\|f\|_{0, \alpha} \text { für } m=0 \text { und }\|f \circ g\|_{m, \alpha} \leq C\|f\|_{m, \alpha} \text { für } m>0 \text {, }
$$

wobei die Konstante $C$ von $m, \alpha, \Omega_{1}, \Omega_{2}$ und $g$ abhängt.

Beweis. Sei zunächst $m=0$, dann gilt

$$
\begin{aligned}
\|f \circ g\|_{\alpha^{2}} & =\|f \circ g\|_{\infty}+|f \circ g|_{\alpha^{2}} \\
& \leq\|f\|_{\infty}+\sup _{x \neq y} \frac{|f(g(x))-f(g(y))|}{|x-y|^{\alpha^{2}}} \\
& \leq\|f\|_{\infty}+|f|_{\alpha} \sup _{x \neq y}\left(\frac{|g(x)-g(y)|}{|x-y|^{\alpha}}\right)^{\alpha} \\
& \leq\|f\|_{\infty}+|f|_{\alpha}|g|_{\alpha}^{\alpha} \leq C\|f\|_{\alpha} .
\end{aligned}
$$

Für $m \geq 1$ werden wir den Mittelwertsatz für die Abbildung $g$ benutzen. Hier benutzen wir die Konvexität des Gebietes $\Omega_{1}$. Für $m=1$ ergibt sich:

$$
\begin{aligned}
\|f \circ g\|_{1, \alpha} & =\|f \circ g\|_{1}+|(\nabla f) \circ g \nabla g|_{\alpha} \\
& \leq\|f\|_{1}\|g\|_{1}+|(\nabla f) \circ g|_{\alpha}\|g\|_{\infty}+\|(\nabla f) \circ g\|_{\infty}|\nabla g|_{\alpha} .
\end{aligned}
$$

Für die Halbnorm erhalten wir:

$$
\begin{aligned}
|(\nabla f) \circ g|_{\alpha, \overline{\Omega_{1}}} & =\sup _{\substack{x, y \in \overline{\Omega_{1}} \\
x \neq y}} \frac{|\nabla f(g(x))-\nabla f(g(y))|}{|x-y|^{\alpha}} \\
& \leq|\nabla f|_{\alpha, \overline{\Omega_{2}}} \sup _{\substack{x, y \in \overline{\Omega_{1}} \\
x \neq y}}\left(\frac{|g(x)-g(y)|}{|x-y|}\right)^{\alpha} \\
& \leq|\nabla f|_{\alpha, \overline{\Omega_{2}}}\|\nabla g\|_{\infty, \mathbb{R}^{d}}^{\alpha} .
\end{aligned}
$$


Somit ist die Behauptung für $m=1$ bewiesen. Nun erfolgt der Induktionsschritt, d.h. für $m \geq 1$ sei die Behauptung bewiesen, dann gilt:

$$
\begin{aligned}
\|f \circ g\|_{m+1, \alpha} & =\|f \circ g\|_{m+1}+\left|\nabla^{m+1}(f \circ g)\right|_{\alpha} \\
& \leq C\|f\|_{m+1}+\left|\nabla^{m}[(\nabla f) \circ g \cdot \nabla g]\right|_{\alpha} \\
& \leq C\|f\|_{m+1}+C \sum_{l=0}^{m}\left(\begin{array}{c}
m \\
l
\end{array}\right)\left\|\nabla^{l}[(\nabla f) \circ g]\right\|_{\alpha}
\end{aligned}
$$

Hierbei wurde die Leibniz-Regel verwendet. Nun sind die Terme der Form $\| \nabla^{l}[(\nabla f) \circ$ $g] \|_{\alpha}$ für $l=0, \ldots, m$ zu untersuchen. Für $l=m$ geht man wie bei $m=1$ vor. Für $0 \leq l \leq m-1$ wendet man den Mittelwertsatz auf $\nabla f$ an (hier geht die Konvexität von $\Omega_{2}$ ein). Insgesamt ergibt sich daraus die Behauptung.

Für zwei kompakte Mengen $A, B \subset \mathbb{R}^{d}$ sei $\mathrm{d}_{H}(A, B)$ der Hausdorff-Abstand (vgl. [22]). Nun beweisen wir eine Aussage über Fortsetzbarkeit von Funktionen.

Lemma 2.2. Sei $\Omega$ ein $C^{m, \alpha}$-Gebiet $(m \in \mathbb{N})$. Dann existieren Operatoren $E_{\Omega} \in L\left(C^{m, \alpha}(\bar{\Omega}), C^{m, \alpha}\left(\mathbb{R}^{d}\right)\right)$ und $E_{n, \partial \Omega} \in L\left(C^{m, \alpha}(\partial \Omega), C^{m, \alpha}\left(\mathbb{R}^{d}\right)\right)$, so daß für alle $u \in C^{m, \alpha}(\bar{\Omega})$ und $v \in C^{m, \alpha}(\partial \Omega)$ gilt: $\left.E_{\Omega} u\right|_{\Omega}=u,\left.E_{n, \partial \Omega} v\right|_{\partial \Omega}=v$ und $\mathrm{d}_{H}\left(\operatorname{supp}\left(E_{n, \partial \Omega} v\right), \partial \Omega\right) \leq 1 / n$.

Beweis. Zunächst zum Operator $E_{\Omega}$. Der Nachweis erfolgt durch Induktion über $m$. Sei hierzu $u \in C^{1, \alpha}(\bar{\Omega})$. Nach Voraussetzung existiert für $x_{0} \in \partial \Omega$ eine offene Umgebung $V_{x_{0}} \subset \mathbb{R}^{d}$ von $x_{0}$ und ein $C^{1, \alpha}$-Diffeomorphismus $\psi_{x_{0}}: V_{x_{0}} \rightarrow B:=\left\{x \in \mathbb{R}^{d}:\|x\|<1\right\}$ mit $\psi_{x_{0}}\left(V_{x_{0}} \cap \bar{\Omega}\right)=\left\{x \in B: x_{d} \geq 0\right\}=: B_{+}$und $\psi_{x_{0}}\left(V_{x_{0}} \cap \Omega_{e}\right)=\left\{x \in B: x_{d}<0\right\}=$ : $B_{-}$. Für $y \in B$ definieren wir

$$
\tilde{u}(y):= \begin{cases}u \circ \psi_{x_{0}}^{-1}(y) & \text { für } y \in B_{+}, \\ \sum_{i=1}^{m+1} c_{i} u \circ \psi_{x_{0}}^{-1}\left(y_{1}, \ldots, y_{d-1},-y_{d} / i\right) & \text { für } y \in B_{-} .\end{cases}
$$

Die noch freien Konstanten $c_{i}$ werden als Lösung des linearen Gleichungssystems

$$
\sum_{i=1}^{m+1} c_{i}(-1 / i)^{k}=1 \text { für } k=0, \ldots, m
$$

gewählt. (Die Determinante der linearen Abbildung ist eine Vandermondsche Determinante und hat den Wert $\Pi_{1 \leq i<j<m+1}(1 / i-1 / j)$ - vgl. [35], Seite 126 - das Gleichungssystem ist somit stets eindeutig lösbar.)

$\mathrm{Zu}$ zeigen ist nun $\tilde{u} \in C^{1, \alpha}(B)$. Offensichtlich gilt $\left.\tilde{u}\right|_{B_{+}} \in C^{1}\left(B_{+}\right)$und $\left.\tilde{u}\right|_{B_{-}} \in$ $C^{1}\left(B_{-}\right)$. Sei nun $y \in B_{+}$mit $y_{d}=0$ und $\left(y^{(n)}\right) \subset B_{-}$mit $\lim y^{(n)}=y$, dann folgt aus $\sum_{i=1}^{m+1} c_{i}=1$ der Grenzwert $\lim \tilde{u}\left(y^{(n)}\right)=\tilde{u}(y)$ und aus $\sum_{i=1}^{m+1}\left(-c_{i} / i\right)=1$ ergibt sich $\lim \nabla \tilde{u}\left(y^{(n)}\right)=\nabla \tilde{u}(y)$ und somit $\tilde{u} \in C^{1}(B)$. 
Noch zu zeigen ist also $|\nabla \tilde{u}|_{\alpha}<\infty$. Sei nun $y \in B_{+}, z \in B_{-}$und $z^{(i)}:=$ $\left(z_{1}, \ldots, z_{d-1},-z_{d} / i\right)$ (d.h. $\left.z^{(i)} \in B_{+}\right)$, dann gilt wegen $\sum_{i=1}^{m+1}\left(-c_{i} / i\right)=1$ :

$$
|\nabla \tilde{u}(y)-\nabla \tilde{u}(z)|=\left|\sum_{i=1}^{m+1} \frac{-c_{i}}{i}\left(\nabla u \circ \psi_{x_{0}}^{-1}(y) \cdot \nabla \psi_{x_{0}}^{-1}(y)-\nabla u \circ \psi_{x_{0}}^{-1}\left(z^{(i)}\right) \cdot \nabla \psi_{x_{0}}^{-1}\left(z^{(i)}\right)\right)\right| .
$$

Wegen $\left\|\nabla \psi_{x_{0}}^{-1}\right\|_{\alpha}<\infty$ ist zum Nachweis von $|\nabla \tilde{u}|_{\alpha}<\infty$ die Ungleichung

$$
\left|\nabla u \circ \psi_{x_{0}}^{-1}(y)-\nabla u \circ \psi_{x_{0}}^{-1}\left(z^{(i)}\right)\right| \leq c|y-z|^{\alpha}
$$

hinreichend (hier wird benutzt, daß das Produkt von Hölder-stetigen Funktionen wieder Hölder-stetig ist).

$$
\begin{aligned}
\left|\nabla u \circ \psi_{x_{0}}^{-1}(y)-\nabla u \circ \psi_{x_{0}}^{-1}\left(z^{(i)}\right)\right| & \leq|\nabla u|_{\alpha}\left|\psi_{x_{0}}^{-1}(y)-\psi_{x_{0}}^{-1}\left(z^{(i)}\right)\right|^{\alpha} \\
& \leq|\nabla u|_{\alpha}|| \nabla \psi_{x_{0}}^{-1} \|_{\infty, B}^{\alpha}\left|y-z^{(i)}\right|^{\alpha} .
\end{aligned}
$$

Hierbei wurde bei der letzten Ungleichung der Mittelwertsatz (vgl. z.B. [13], Seite 52f.) unter Verwendung der Konvexität von $B$ benutzt. Beachtet man nun die Ungleichung $\left|y_{d}+z_{d} / i\right|<\left|y_{d}-z_{d} / i\right| \leq\left|y_{d}-z_{d}\right|$, so folgt

$$
|\nabla \tilde{u}(y)-\nabla \tilde{u}(z)| \leq C\|u\|_{1, \alpha}|y-z|^{\alpha}, \quad y \in B_{+}, z \in B_{-}
$$

Die Konstante $C$ hängt hierbei nicht von $u$ ab. In den anderen Fällen (d.h. $y, z \in B_{+}$ oder $y, z \in B_{-}$) ist die Ungleichung $|\nabla \tilde{u}|_{\alpha}<\infty$ offensichtlich, so daß die Behauptung für $m=1$ gezeigt ist.

Sei nun $K:=B_{r}\left(x_{0}\right)$ eine Kugel um $x_{0}$ mit $\psi_{x_{0}}(K) \Subset B_{1}$. Dann gilt $w_{x_{0}}:=\tilde{u} \circ \psi_{x_{0}} \in$ $C^{1, \alpha}(K)$ mit $\left\|w_{x_{0}}\right\|_{1, \alpha} \leq c\|u\|_{1, \alpha}$ und $\left.w_{x_{0}}\right|_{K \cap \Omega}=u$. Die Hölder-Stetigkeit von $w_{x_{0}}$ folgt aus Lemma 2.1. Da $\partial \Omega$ kompakt ist, findet man somit eine endliche Überdeckung von $\partial \Omega$ durch Kugeln $\Omega_{j}:=B_{r_{j}}\left(x_{j}\right)$ mit $x_{j} \in \partial \Omega$ und Funktionen $w_{j} \in C^{1, \alpha}\left(\overline{\Omega_{j}}\right)$ mit $\left.w_{j}\right|_{\Omega_{j} \cap \Omega}=u$ und $\left\|w_{j}\right\|_{1, \alpha, \Omega_{j}} \leq c\|u\|_{1, \alpha, \Omega_{j} \cap \Omega}$. Sei weiterhin $\Omega_{0} \Subset \Omega$ mit $\Omega \Subset \bigcup_{j=0}^{J} \Omega_{j}$ (vgl. die Abbildung 2.3) und $\left\{\eta_{j}\right\}_{j=0}^{J}$ eine unendlich oft differenzierbare Zerlegung der Eins zu diesen Gebieten (d.h. $\eta_{j} \in C_{0}^{\infty}\left(\mathbb{R}^{d}\right)$, supp $\eta_{j} \subset \Omega_{j}, j=0, \ldots, J ; \eta_{j} \geq 0$ und $\sum_{j=0}^{J} \eta_{j}=1$ in $\Omega$. Für einen Beweis der Existenz einer solchen Zerlegung vgl. [62], Seite 61). Hiermit definiere man die Funktion $w: \mathbb{R}^{d} \rightarrow \mathbb{C}$ durch

$$
w:=u \eta_{0}+\sum_{j=1}^{J} w_{j} \eta_{j}
$$

wobei wir die Konvention $w_{j}(x)=0$ für $x \notin \bar{\Omega}$ benutzen. Die Funktion $w$ hat nun genau die geforderten Eigenschaften und aus der Linearität der Abbildung $u \mapsto w$ folgt die Existenz des Operators $E_{\Omega}$.

Nun erfolgt der Induktionsschluß von $m$ nach $m+1$, wir setzen also die Gültigkeit des Satzes für $m$ voraus. Der Nachweis von $\tilde{u} \in C^{m+1}(B)$ (wobei $\tilde{u}$ in (2.1) definiert wurde) 


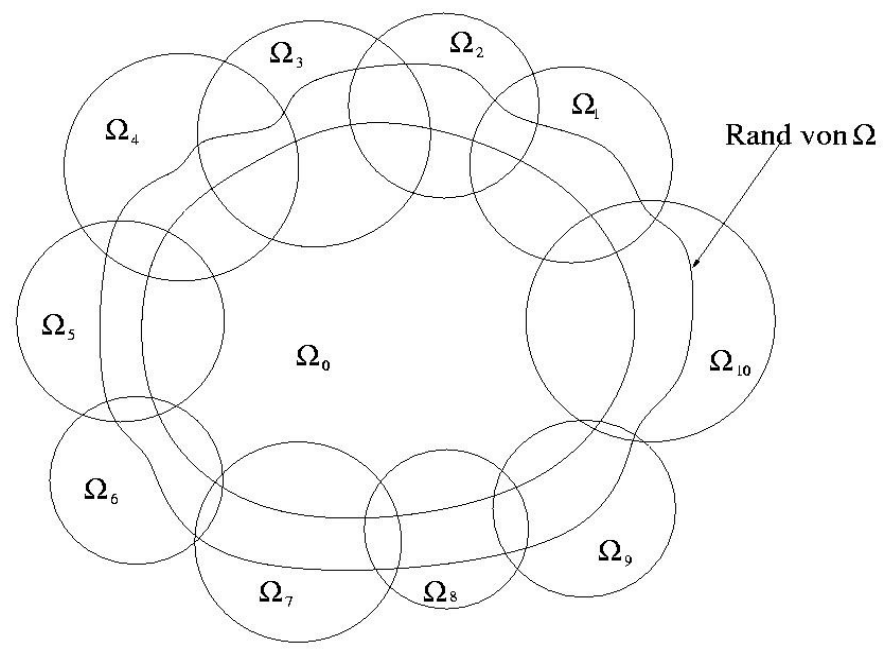

Abbildung 2.3: Fortsetzung

erfolgt wie bei $\tilde{u} \in C^{1}(B)$. Für die Aussage $\left|\nabla^{m+1} \tilde{u}\right|_{\alpha}<\infty$ sind nun Terme von der Form $\left(\nabla^{l} u\right) \circ \psi_{x_{0}}^{-1}(y)$ (für $y \in B_{+}$und entsprechende Terme für $y \in B_{-}$) für $0 \leq l \leq m+1$ zu untersuchen. Für $l=m+1$ geht man wie bei $m=1$ vor, sonst verwendet man den Mittelwertsatz für $\nabla^{l} u$. Hier ist zu beachten, daß der Mittelwertsatz in $V_{x_{0}} \cap \bar{\Omega}$ gebraucht wird, dieses Gebiet aber nicht notwendigerweise konvex ist. Daher setzt man $\nabla^{l} u$ zunächst unter Verwendung der Induktionsvoraussetzung fort und benutzt den Mittelwertsatz für die fortgesetzte Funktion. Damit ist für den Operator $E_{\Omega}$ alles gezeigt.

Nun zum Operator $E_{n, \partial \Omega}$, wobei die gleiche Notation wie beim ersten Teil des Beweises verwendet wird. Sei $u \in C^{m, \alpha}(\partial \Omega)$, d.h. $u \circ \psi_{x_{0}}^{-1} \in C^{m, \alpha}\left(\partial B_{+} \cap \partial B_{-}\right)$. Wir definieren

$$
\tilde{u}\left(y_{1}, \ldots, y_{d}\right):=u \circ \psi_{x_{0}}^{-1}\left(y_{1}, \ldots, y_{d-1}, 0\right)
$$

und erhalten $\tilde{u} \in C^{m, \alpha}(B)$ mit $\|\tilde{u}\|_{m, \alpha} \leq c\|u\|_{m, \alpha}$. Für die Funktion $w_{x_{0}}:=\tilde{u} \circ \psi_{x_{0}}$ gilt dann $\left\|w_{x_{0}}\right\|_{m, \alpha} \leq c\|u\|_{m, \alpha}$ und $\left.w_{x_{0}}\right|_{K \cap \partial \Omega}=u$. Nun kann genau wie im ersten Teil des Beweises argumentiert werden, so daß wir einen Operator $E_{\partial \Omega} \in L\left(C^{m, \alpha}(\partial \Omega), C^{m, \alpha}\left(\mathbb{R}^{d}\right)\right)$ mit $\left.E_{\partial \Omega} u\right|_{\partial \Omega}=u$ erhalten. Wähle nun Gebiete $\Omega_{1} \Subset \Omega_{2}$ mit $\partial \Omega \subset \Omega_{1}$ und $\operatorname{dist}\left(\Omega_{2}, \partial \Omega\right) \leq 1 / n$ und eine Funktion $\eta \in C_{0}^{\infty}\left(\mathbb{R}^{d}\right) \operatorname{mit} \operatorname{supp}(\eta) \subset \Omega_{2}$ und $\left.\eta\right|_{\Omega_{1}}=1$, dann hat $E_{n, \partial \Omega}$ mit

$$
E_{n, \partial \Omega} u:=\eta E_{\partial \Omega} u
$$

die gewünschten Eigenschaften.

Mit Hilfe dieses Lemmas beweisen wir, daß die Komposition von Hölder-stetigen Funktionen auch in nichtkonvexen Gebieten wieder Hölder-stetig ist.

Korollar 2.3. Seien $\Omega_{1}, \Omega_{2} C^{m, \alpha}$-Gebiete, $f \in C^{m, \alpha}\left(\overline{\Omega_{2}}\right)$ und $g \in C^{m, \alpha}\left(\overline{\Omega_{1}}, \overline{\Omega_{2}}\right)$, dann folgt

$$
f \circ g \in C^{0, \alpha^{2}}\left(\overline{\Omega_{1}}\right) \text { für } m=0 \text { und } f \circ g \in C^{m, \alpha}\left(\overline{\Omega_{1}}\right) \text { für } m>0 \text {. }
$$


Weiterhin gelten die Abschätzungen

$$
\|f \circ g\|_{0, \alpha^{2}} \leq C\|f\|_{0, \alpha} \text { für } m=0 \text { und }\|f \circ g\|_{m, \alpha} \leq C\|f\|_{m, \alpha} \text { für } m>0 \text {, }
$$

wobei die Konstante $C$ von $m, \alpha, \Omega_{1}, \Omega_{2}$ und $g$ abhängt.

Beweis. Der Beweis verläuft wie in Lemma 2.1. Da die Gebiete nun nicht notwendigerweise konvex sind, verwendet man für den Mittelwertsatz Fortsetzungen der Funktionen $f$ und $g$. 


\section{Potentialoperatoren}

In diesem Kapitel werden eine Reihe von benötigten Hilfsmitteln bereitgestellt. Insbesondere beweisen wir Abbildungseigenschaften von Einfachschicht-, Doppelschichtund Volumenpotential in Hölder-Räumen. Diese werden dann zur Lösung von verschiedenen Randwertproblemen verwendet. Um die Darstellung möglichst übersichtlich zu halten, beschränken wir uns auf $C^{m, \alpha}$-Gebiete mit $m \in \mathbb{N}_{\geq 2}$ und verzichten auf Aussagen bei geringerer Randregularität.

\subsection{Einfach- und Doppelschichtpotentialoperator}

Zunächst untersuchen wir den Einfachschichtpotentialoperator $\mathcal{S}$, mit

$$
\mathcal{S} \varphi(x):=\int_{\partial \Omega} \Phi(x, y) \varphi(y) \mathrm{ds}(y), \quad x \in \Omega
$$

und den Doppelschichtpotentialoperator $\mathcal{K}$, mit

$$
\mathcal{K} \varphi(x):=\int_{\partial \Omega} \frac{\partial \Phi(x, y)}{\partial \nu(y)} \varphi(y) \operatorname{ds}(y), \quad x \in \Omega
$$

Hierbei sei das Gebiet $\Omega$ von der im letzten Kapitel besprochenen Form, d.h. $\Omega$ kann sowohl beschränkt, als auch unbeschränkt sein. Die Operatoren $\tilde{\mathcal{S}}$ und $\tilde{\mathcal{K}}$ bezeichnen die entsprechenden Operatoren für eine Wellenzahl $\tilde{\kappa} \neq \kappa$ und die Operatoren $\mathcal{S}_{0}$ und $\mathcal{K}_{0}$ die Operatoren mit der Grundlösung $\Phi_{0}$ für die Laplacegleichung. Es gelten folgende Abbildungseigenschaften:

Satz 3.1. Sei $m \in \mathbb{N}_{\geq 2}$ und $\partial \Omega \in C^{m, \alpha}$, dann folgt

1. $\mathcal{S} \in L\left(C^{m-1, \alpha}(\partial \Omega), C^{m, \alpha}(\bar{\Omega})\right), \mathcal{K} \in L\left(C^{m, \alpha}(\partial \Omega), C^{m, \alpha}(\bar{\Omega})\right)$,

2. $(\mathcal{S}-\tilde{\mathcal{S}}) \in L\left(C(\partial \Omega), C^{2, \alpha}\left(\mathbb{R}^{d}\right)\right),(\mathcal{K}-\tilde{\mathcal{K}}) \in L\left(C(\partial \Omega), C^{1, \alpha}\left(\mathbb{R}^{d}\right)\right)$, $(\mathcal{S}-\tilde{\mathcal{S}}) \in L\left(C^{m-1}(\partial \Omega), C^{m+1, \alpha}(\bar{\Omega})\right),(\mathcal{K}-\tilde{\mathcal{K}}) \in L\left(C^{m}(\partial \Omega), C^{m+1, \alpha}(\bar{\Omega})\right)$.

3. Für $\Omega^{\prime} \Subset \Omega$ und $l \in \mathbb{N}$ gilt $\mathcal{S}, \mathcal{K} \in L\left(C(\partial \Omega), C^{l, \alpha}\left(\overline{\Omega^{\prime}}\right)\right)$.

4. Der Operator $\mathcal{K}_{0}$ hat die gleichen Abbildungseigenschaften wie $\mathcal{K}$ und $\mathcal{S}_{0}$ hat die gleichen Abbildungseigenschaften wie $\mathcal{S}$, im $\mathbb{R}^{2}$ allerdings nur für beschränktes $\Omega$.

Beweis. Eine ähnliche Aussage wurde von Kirsch für den dreidimensionalen Fall bewiesen (vgl. die Beweise von Theorem 2.10 in [29] und Theorem 3.2 und 3.3 in [30]). 
Der Beweis besteht aus zwei Teilen. Zunächst ist zu zeigen, daß die Ableitungen von Einfach - und Doppelschichtpotential wieder durch Einfach- und Doppelschichtpotential mit geeigneten Dichten darstellbar sind. Diese Rekursionsformeln nutzt man dann für einen induktiven Nachweis der Aussagen.

1. Zunächst zu den Behauptungen bzgl. $\mathcal{S}$ und $\mathcal{K}$. Die Aussagen $\mathcal{S} \in$ $L\left(C^{0, \alpha}(\partial \Omega), C^{1, \alpha}(\bar{\Omega})\right)$ und $\mathcal{K} \in L\left(C^{j, \alpha}(\partial \Omega), C^{j, \alpha}(\bar{\Omega})\right), j=0,1$, werden im dreidimensionalen Fall in [6] (Theorem 2.16, Theorem 2.17 und Theorem 2.23) und im $\mathbb{R}^{2}$ in [57], Satz 2.26, Satz 2.27 und Satz 2.35, bewiesen. Die Beweise sind dort zwar für Gebiete mit zusammenhängenden Rändern geführt worden, da es sich bei der Hölder-Stetigkeit jedoch um eine lokale Eigenschaft handelt (vgl. Lemma 7.3 aus [37]), können sie aber in dieser Situation genau analog hergeleitet werden.

Nun benutzen wir für den zweidimensionalen Fall die Identitäten

$$
\begin{aligned}
& \nabla \mathcal{S} \varphi=-\mathcal{K}(\nu \varphi)+\mathcal{S} \frac{\mathrm{d}}{\mathrm{ds}}(\varphi \vartheta) \operatorname{mit} \vartheta=\left(-\nu_{2}, \nu_{1}\right), \quad \varphi \in C^{1}(\partial \Omega), \\
& \nabla \mathcal{K} \varphi=\kappa^{2} \mathcal{S}(\nu \varphi)+\left(\begin{array}{c}
\nabla_{2} \\
-\nabla_{1}
\end{array}\right) \mathcal{S} \frac{\mathrm{d} \varphi}{\mathrm{ds}}, \quad \varphi \in C^{1, \alpha}(\partial \Omega),
\end{aligned}
$$

die in Theorem 2.1 in [28] bewiesen wurden (die Gleichung 3.4 wurde im Verlauf des Beweises hergeleitet).

Als Induktionsvoraussetzung gelte $\mathcal{S} \in L\left(C^{m-2, \alpha}(\partial \Omega), C^{m-1, \alpha}(\bar{\Omega})\right)$ und $\mathcal{K} \in$ $L\left(C^{m-1, \alpha}(\partial \Omega), C^{m-1, \alpha}(\bar{\Omega})\right)$. Für $\partial \Omega \in C^{m, \alpha}$ (d.h. insbesondere $\nu, \vartheta \in$ $\left.C^{m-1, \alpha}(\partial \Omega)\right)$ folgt aus (3.3) $\nabla \mathcal{S} \in L\left(C^{m-1, \alpha}(\bar{\Omega}), C^{m-1, \alpha}\left(\partial \Omega, \mathbb{R}^{2}\right)\right)$ und daher $\mathcal{S} \in L\left(C^{m-1, \alpha}(\bar{\Omega}), C^{m, \alpha}(\bar{\Omega})\right)$. Aus der Gleichung (3.4) folgt analog die Regularitätsbeziehung $\mathcal{K} \varphi \in L\left(C^{m, \alpha}\left(\bar{\Omega}, C^{m, \alpha}(\bar{\Omega})\right)\right.$. Der dreidimensionale Fall wird entsprechend bewiesen. Hier verwendet man die Rekursionsformeln aus dem Beweis von Theorem 2.10 aus [29].

2. Nun zu den beiden Behauptungen bzgl. der Differenzen von zwei Potentialen. Exemplarisch soll dies für den zweidimensionalen Fall bewiesen werden. Der Kern $k$ von $\nabla(\mathcal{S}-\tilde{\mathcal{S}})$ ist gegeben durch:

$$
k(x, y)=(x-y)(f(|x-y|)+\ln |x-y| g(|x-y|)),
$$

wobei $f$ und $g$ analytische Funktionen mit $g(0) \neq 0$ sind (vgl. [57], Satz 2.23). Nun betrachten wir nur noch den Term $(x-y) \ln |x-y|$, da die restlichen Terme eine höhere Regularität aufweisen. Es gilt:

$$
\nabla_{x}(x-y) \ln |x-y|=\mathbb{1} \ln |x-y|+\frac{(x-y) \odot(x-y)}{|x-y|^{2}} .
$$

Hierbei ist $\mathbb{1}$ die Identitätsmatrix. Jetzt benutzt man Satz 3.7. Beide Summanden erfüllen die Voraussetzung dieses Satzes und somit folgt $(\mathcal{S}-\tilde{\mathcal{S}}) \in$ $L\left(C(\partial \Omega), C^{2, \alpha}\left(B_{R}\right)\right)$ und damit $(\mathcal{S}-\tilde{\mathcal{S}}) \in L\left(C(\partial \Omega), C^{2, \alpha}\left(\mathbb{R}^{2}\right)\right)$. 
Der Kern von $k$ von $\nabla(\mathcal{K}-\tilde{\mathcal{K}})$ hat folgende Gestalt (vgl. [57], Satz 2.30):

$$
k(x, y)=\left((x-y) \odot \frac{(x-y) \cdot \nu(y)}{|x-y|^{2}}+\nu(y)\right)(f(|x-y|)+\ln |x-y| g(|x-y|)),
$$

wobei $f$ und $g$ analytische Funktionen mit $g(0) \neq 0$ sind. Nun ist die Funktion

$$
w(x, y):=\ln |x-y|(x-y) \odot \frac{(x-y) \cdot \nu(y)}{|x-y|^{2}}
$$

zu untersuchen $(\ln |x-y|$ wurde bereits behandelt und die restlichen Terme des Kerns weisen eine höhere Regularität auf). Mit der gleichen Beweisidee wie in Lemma 3.5 kann man nachweisen, daß $w$ die Voraussetzungen von Satz 3.7 erfüllt und somit ergibt sich $(\mathcal{K}-\tilde{\mathcal{K}}) \in L\left(C(\partial \Omega), C^{1, \alpha}\left(\mathbb{R}^{2}\right)\right)$.

Aus den Gleichungen (3.3) und (3.4) folgt weiterhin:

$$
\begin{aligned}
& \nabla(\mathcal{S}-\tilde{\mathcal{S}}) \varphi=(\tilde{\mathcal{K}}-\mathcal{K})(\nu \varphi)+(\mathcal{S}-\tilde{\mathcal{S}}) \frac{\mathrm{d}}{\mathrm{ds}}(\varphi \vartheta), \\
& \nabla(\mathcal{K}-\tilde{\mathcal{K}}) \varphi=\left(\kappa^{2} \mathcal{S}-\tilde{\kappa}^{2} \tilde{\mathcal{S}}\right)(\nu \varphi)+\left(\begin{array}{c}
\nabla_{2} \\
-\nabla_{1}
\end{array}\right)(\mathcal{S}-\tilde{\mathcal{S}}) \frac{\mathrm{d} \varphi}{\mathrm{ds}}
\end{aligned}
$$

wobei diese Gleichungen für $\varphi \in C^{1}(\partial \Omega)$ gültig sind. Genauso wie beim ersten Punkt des Beweises können aus diesen Gleichungen nun induktiv die Regularitätsaussagen nachgewiesen werden.

3. Die dritte Behauptung folgt wegen $\Omega^{\prime} \Subset \Omega$ direkt aus der Analytizität der Grundlösung.

4. Nun zur letzten Aussage. Da die Singularitäten der Funktionen $\Phi$ und $\Phi_{0}$ gleich sind, können die Aussagen für $\mathcal{S}_{0}$ und $\mathcal{K}_{0}$ völlig analog geführt werde. $\mathrm{Zu}$ beachten ist nur, daß das Einfachschichtpotential der Laplace-Gleichung im $\mathbb{R}^{2}$ nicht beschränkt ist; daher lassen sich die Aussagen nur für beschränkte Gebiete übertragen.

Als nächstes untersuchen wir für $x \in \partial \Omega$ folgende Integraloperatoren:

$$
\begin{aligned}
S \varphi(x) & :=\int_{\partial \Omega} \Phi(x, y) \varphi(y) \mathrm{ds}(y), \\
K \varphi(x) & :=\int_{\partial \Omega} \frac{\partial \Phi(x, y)}{\partial \nu(y)} \varphi(y) \mathrm{ds}(y), \\
K^{*} \varphi(x) & :=\int_{\partial \Omega} \frac{\partial \Phi(x, y)}{\partial \nu(x)} \varphi(y) \mathrm{ds}(y), \\
T \varphi(x) & :=\frac{\partial}{\partial \nu(x)} \int_{\partial \Omega} \frac{\partial \Phi(x, y)}{\partial \nu(y)} \varphi(y) \operatorname{ds}(y) .
\end{aligned}
$$


Auch hier betrachten wir die Operatoren $\tilde{S}, \tilde{K}, \tilde{K}^{*}$ und $\tilde{T}$, d.h. die entsprechenden Operatoren für eine Wellenzahl $\tilde{\kappa} \neq k$ und Operatoren mit dem Index 0 für die LaplaceGleichung. Diese Operatoren haben folgende Eigenschaften:

Satz 3.2. Für $\partial \Omega \in C^{m, \alpha}, m \in \mathbb{N}_{\geq 2}$ gilt

1. Für $\varphi \in C(\partial \Omega), \psi \in C^{1, \alpha}(\partial \Omega)$ ergibt sich $\mathcal{S} \varphi_{ \pm}=S \varphi, \frac{\partial \mathcal{S} \varphi}{\partial \nu}{ }_{ \pm}=K^{*} \varphi \mp \frac{1}{2} \varphi$, $\mathcal{K} \varphi_{ \pm}=K \varphi \pm \frac{1}{2} \varphi$ und $\frac{\partial \mathcal{K} \psi}{\partial \nu}{ }_{ \pm}=T \psi$.

2. $S, K \in L\left(C^{m-1, \alpha}(\partial \Omega), C^{m, \alpha}(\partial \Omega)\right), K^{*} \in L\left(C^{m-2, \alpha}(\partial \Omega), C^{m-1, \alpha}(\partial \Omega)\right)$, $T \in L\left(C^{m, \alpha}(\partial \Omega), C^{m-1, \alpha}(\partial \Omega)\right)$,

3. $S-\tilde{S} \in \begin{cases}L\left(C(\partial \Omega), C^{2, \alpha}(\partial \Omega)\right) & m=2, \\ L\left(C^{m-3, \alpha}(\partial \Omega), C^{m, \alpha}(\partial \Omega)\right) & m \geq 3,\end{cases}$

$K-\tilde{K} \in L\left(C^{m-2, \alpha}(\partial \Omega), C^{m, \alpha}(\partial \Omega)\right)$,

$K^{*}-\tilde{K}^{*} \in \begin{cases}L\left(C(\partial \Omega), C^{1, \alpha}(\partial \Omega)\right) & m=2, \\ L\left(C^{m-3, \alpha}(\partial \Omega), C^{m-1, \alpha}(\partial \Omega)\right) & m \geq 3,\end{cases}$

$T-\tilde{T} \in L\left(C^{m-2, \alpha}(\partial \Omega), C^{m-1, \alpha}(\partial \Omega)\right)$.

4. Die Operatoren $S_{0}, K_{0}, K_{0}^{*}$ und $T_{0}$ haben die gleichen Abbildungseigenschaften wie $S, K, K^{*}$ und $T$.

Beweis. Auch dieser Satz wurde in ähnlicher Form von Kirsch für den dreidimensionalen Fall bewiesen (vgl. die Beweise von Theorem 2.10 in [29] und Theorem 3.2, 3.3 und 4.4 in [30]). Die Beweisidee ist die gleiche wie bei Satz 3.1, d.h. man schließt induktiv mit Hilfe von geeigneten Rekursionsformeln. Nun zu den einzelnen Punkten:

1. Bei diesen Gleichungen, die bereits für $C^{2}$-Ränder gültig sind, handelt es sich um die klassischen Sprungbeziehungen der Potentialtheorie. Beweise hierfür sind in der Literatur reichlich vorhanden. Vgl. z.B. [6] (Theorem 2.12, 2.13, 2.19 und 2.23) für den dreidimensionalen Fall. $\operatorname{Im} \mathbb{R}^{2}$ wurden die Aussagen z.B. in [57] (Sätze 2.22, 2.24, 2.29 und 2.35) bewiesen.

2. Wir beweisen nun die Aussagen für die Operatoren $S, K, K^{*}$ und $T . S$, $K \in L\left(C^{0, \alpha}(\partial \Omega), C^{1, \alpha}(\partial \Omega)\right)$ wurde $\operatorname{im} \mathbb{R}^{3}$ in $[6]$, Theorem 2.17 und 2.22 bewiesen und im $\mathbb{R}^{2}$ in Satz 2.27 und 2.34 in [57]. $K^{*} \in L\left(C^{0, \alpha}(\partial \Omega), C^{1, \alpha}(\partial \Omega)\right)$ wurde im $\mathbb{R}^{3}$ in [29], Theorem 2.8, bewiesen. Den analogen Beweis für die zweidimensionale Situation werden wir in Lemma 3.3 skizzieren. Für die Aussage $T \in L\left(C^{1, \alpha}(\partial \Omega), C^{0, \alpha}(\partial \Omega)\right)$ vgl. [6], Theorem 2.23 und [57], Satz 2.35.

Die Beweise für die allgemeine Situation erfolgen wiederum durch Induktion. Im $\mathbb{R}^{3}$ wurde dies in [29], Theorem 2.11 und 2.12, bewiesen. Grundlage für den Beweis sind Rekursionsformeln, die in Theorem 2.10 bewiesen wurden. Im zweidimensionalen ist die Vorgehensweise ähnlich. Aus der Beziehung $S \varphi=\mathcal{S} \varphi_{ \pm}$ergibt sich, 
daß sich die Regularität von $\mathcal{S}$ auf $S$ überträgt. Somit folgt die Behauptung für $S$ aus Satz 3.1.

Für den Operator $K^{*}$ und $\varphi \in C^{1}(\partial \Omega)$ wurde in Theorem 2.1 in [28] die Gleichung

$$
K^{*} \varphi=-K(\varphi \nu) \cdot \nu+S\left(\varphi \frac{\mathrm{d} \vartheta}{\mathrm{ds}}\right) \cdot \nu+S\left(\frac{\mathrm{d} \varphi}{\mathrm{ds}} \vartheta\right) \cdot \nu
$$

bewiesen. Der Integraloperator

$$
A \varphi(x):=\int_{\partial \Omega} \nabla_{x} \Phi(x, y) \varphi(y) \odot \vartheta(y) \cdot \nu(x) \operatorname{ds}(y) .
$$

erfüllt $A \in L\left(C^{m-2, \alpha}(\partial \Omega), C^{m-1, \alpha}\left(\partial \Omega, \mathbb{R}^{2}\right)\right)$ und

$$
\left(A \frac{\mathrm{d} \varphi}{\mathrm{ds}}\right) \cdot \vartheta=\left(\frac{\mathrm{d}}{\mathrm{ds}} S\left(\frac{\mathrm{d} \varphi}{\mathrm{ds}} \vartheta\right)\right) \cdot \nu
$$

(vgl. Lemma 3.4). Daher folgt aus den Abbildungseigenschaften von $A, S$ und $K$ die Regularitätseigenschaft $\frac{\mathrm{d}}{\mathrm{ds}} K^{*} \in L\left(C^{m-2, \alpha}(\partial \Omega), C^{m-2, \alpha}(\partial \Omega)\right)$ und damit die Behauptung.

Nun zum Operator $K$. Aus $K \varphi=\mathcal{K} \varphi_{ \pm} \mp \frac{1}{2} \varphi$, Gleichung (3.4) und $\frac{\partial \mathcal{S} \varphi}{\partial \nu}{ }_{ \pm}=$ $K^{*} \varphi \mp \frac{1}{2} \varphi$ ergibt sich

$$
\begin{aligned}
\frac{\mathrm{d}}{\mathrm{ds}} K \varphi & =\nabla \mathcal{K} \varphi_{ \pm} \cdot \vartheta \mp \frac{1}{2} \frac{\mathrm{d} \varphi}{\mathrm{ds}} \\
& =\kappa^{2} \mathcal{S}(\varphi \nu)_{ \pm} \cdot \vartheta-K^{*} \frac{\mathrm{d} \varphi}{\mathrm{ds}} \pm \frac{1}{2} \frac{\mathrm{d} \varphi}{\mathrm{ds}} \mp \frac{1}{2} \frac{\mathrm{d} \varphi}{\mathrm{ds}} \\
& =\kappa^{2} S(\varphi \nu) \cdot \vartheta-K^{*} \frac{\mathrm{d} \varphi}{\mathrm{ds}}
\end{aligned}
$$

und daher $K \in L\left(C^{m-1, \alpha}(\partial \Omega), C^{m, \alpha}(\partial \Omega)\right)$.

Aus der Gleichung (3.4) und $\frac{\partial \mathcal{K} \varphi}{\partial \nu}{ }_{ \pm}=T \varphi$ folgt

$$
T \varphi=\kappa^{2} S(\nu \varphi) \cdot \nu+\frac{\mathrm{d}}{\mathrm{ds}} S \frac{\mathrm{d} \varphi}{\mathrm{ds}} .
$$

Diese Gleichung wurde von Maue hergeleitet (vgl. [46]). Die Aussage für den Operator $T$ folgt nun aus den Abbildungseigenschaften von $S$.

3. Nun zu den Behauptungen bzgl. der Differenz von Operatoren. Die Beweise erfolgen nur für den zweidimensionalen Fall. Im $\mathbb{R}^{3}$ kann jeweils entsprechend gefolgert werden (vgl. [30], Theorem 4.4). Die Abbildungseigenschaft $S-\tilde{S} \in L\left(C(\partial \Omega), C^{2, \alpha}(\partial \Omega)\right)$ folgt direkt aus der entsprechenden Regularität 
von $\mathcal{S}-\tilde{\mathcal{S}}$. Für $m=3$ nutzt man die Darstellung (3.5). Differenziert man den Term $(x-y) \cdot \vartheta(x) \ln |x-y|$ nach der Variablen $x$, so ergibt sich:

$$
\begin{aligned}
& \frac{\mathrm{d}}{\mathrm{ds}}(x-y) \cdot \vartheta(x) \ln |x-y| \\
& =\ln |x-y|+(x-y) \cdot \frac{\mathrm{d} \vartheta}{\mathrm{ds}}(x) \ln |x-y|+\left(\frac{(x-y) \cdot \vartheta(x)}{|x-y|}\right)^{2} .
\end{aligned}
$$

Exemplarisch betrachten wir nur noch den letzten Term. Erneutes Differenzieren ergibt:

$$
\begin{aligned}
& \frac{\mathrm{d}}{\mathrm{ds}}\left(\frac{(x-y) \cdot \vartheta(x)}{|x-y|}\right)^{2} \\
& =2 \frac{(x-y) \cdot \vartheta(x)}{|x-y|^{2}}\left(1+(x-y) \cdot \frac{\mathrm{d} \vartheta}{\mathrm{ds}}(x)-\left(\frac{(x-y) \cdot \vartheta(x)}{|x-y|}\right)^{2}\right)=: w(x, y) g(x, y)
\end{aligned}
$$

Die Funktion $w(x, y)$ ist der Kern der Tangentialableitung des Einfachschichtpotentials zur Laplacegleichung und $g(x, y)$ ist eine, in beiden Argumenten Hölderstetige Funktion (vgl. Lemma 3.6). Nun benutzt man die Aufspaltung

$$
w(x, y) g(x, y)=w(x, y)(g(x, y)-g(y, y))-w(x, y) g(y, y) .
$$

Die Hölder-Stetigkeit des ersten Terms zeigt man wie in Satz 2.20 aus [57] und beim zweiten Term nutzt man die Abbildungseigenschaften des Einfachschichtpotentials. Somit folgt die Behauptung. Aus der Gleichung (3.3) folgt für $\varphi \in C^{1}(\partial \Omega)$

$$
\frac{\mathrm{d}}{\mathrm{ds}} S \varphi=\nabla \mathcal{S} \varphi_{ \pm} \cdot \vartheta=S\left(\frac{\mathrm{d}}{\mathrm{ds}}(\varphi \vartheta)\right) \cdot \vartheta-K(\varphi \nu) \cdot \vartheta
$$

Somit ergibt sich:

$$
\frac{\mathrm{d}}{\mathrm{ds}}(S-\tilde{S}) \varphi=(S-\tilde{S})\left(\frac{\mathrm{d}}{\mathrm{ds}}(\varphi \vartheta)\right) \cdot \vartheta-(K-\tilde{K})(\varphi \nu) \cdot \vartheta
$$

und daraus folgt für $m \geq 4$ induktiv die Behauptung.

Nun zum Vorgehen für $K-\tilde{K}$. Hier betrachten wir exemplarisch wieder nur den zweidimensionalen Fall. Zu untersuchen ist der Term $(x-y) \cdot \vartheta(x) \ln |x-y| \frac{(x-y) \cdot \nu(y)}{|x-y|^{2}}$ des Kerns von $\frac{\mathrm{d}}{\mathrm{ds}}(K-\tilde{K})$ für einen Tangentialvektor $\vartheta$ (vgl. die Darstellung (3.6); 
die anderen Terme sind einfacher zu behandeln):

$$
\begin{aligned}
& \frac{\mathrm{d}}{\mathrm{ds}}\left[(x-y) \cdot \vartheta(x) \ln |x-y| \frac{(x-y) \cdot \nu(y)}{|x-y|^{2}}\right] \\
& =\left(\frac{(x-y) \cdot \vartheta(x)}{|x-y|}\right)^{2} \frac{(x-y) \cdot \nu(y)}{|x-y|^{2}} \\
& \quad+\ln |x-y|\left(1+(x-y) \cdot \frac{\mathrm{d} \vartheta}{\mathrm{ds}}(x)\right) \frac{(x-y) \cdot \nu(y)}{|x-y|^{2}} \\
& \quad+\ln |x-y|(x-y) \cdot \vartheta(x)\left(\frac{\vartheta(x) \cdot \nu(y)}{|x-y|^{2}}-2 \frac{(x-y) \cdot \vartheta(x) \odot(x-y) \cdot \nu(y)}{|x-y|^{4}}\right)
\end{aligned}
$$

Der erste Term kann interpretiert werden als Kern des Doppelschichtpotentials, die anderen beiden als Kerne des Einfachschichtpotentials zur Laplacegleichung mit Hölder-stetiger Dichte (vgl. Lemma 3.6 und den Beweis für $S-\tilde{S})$ und somit folgt $K-\tilde{K} \in L\left(C^{0, \alpha}(\partial \Omega), C^{2, \alpha}(\partial \Omega)\right)$. Für $m \geq 3$ benutzt man die Gleichung (3.12):

$$
\frac{\mathrm{d}}{\mathrm{ds}}(K-\tilde{K}) \varphi=\left(\kappa^{2} S-\tilde{\kappa}^{2} \tilde{S}\right)(\varphi \nu) \cdot \vartheta-\left(K^{*}-\tilde{K}^{*}\right) \frac{\mathrm{d} \varphi}{\mathrm{ds}}
$$

und somit folgt auch hier induktiv die Behauptung.

Für $m=2$ benutzt man $K^{*}-\tilde{K}^{*}=\nabla(\mathcal{S}-\tilde{\mathcal{S}})_{ \pm} \cdot \nu$. Für $m=3$ betrachtet man die Kernfunktion und argumentiert wie bei $K-\tilde{K}$. Für $m \geq 4$ verwenden wir die Gleichung (3.11):

$$
\left(K^{*}-\tilde{K}^{*}\right) \varphi=\left[(S-\tilde{S}) \frac{\mathrm{d}}{\mathrm{ds}}(\varphi \vartheta)-(K-\tilde{K})(\varphi \nu)\right] \cdot \nu .
$$

Aus den Abbildungseigenschaften von $S-\tilde{S}$ und $K-\tilde{K}$ folgt dann die Behauptung für $K^{*}-\tilde{K}^{*}$.

Zuletzt nun zu $T-\tilde{T}$. Hier ist für $m=2$ die Gleichung $\nabla(\mathcal{K}-\tilde{\mathcal{K}})_{ \pm} \cdot \nu=T-\tilde{T}$ der Weg zum Ziel, für $m \geq 3$ die Gleichung (3.13), die wir für $\varphi \in C^{m-2, \alpha}(\partial \Omega)$ benutzen:

$$
(T-\tilde{T}) \varphi=\left(\kappa^{2} S-\tilde{\kappa}^{2} \tilde{S}\right)(\varphi \nu) \cdot \nu+\frac{\mathrm{d}}{\mathrm{ds}}(S-\tilde{S}) \frac{\mathrm{d} \varphi}{\mathrm{ds}} .
$$

4. Die Beweise für die Operatoren zur Laplacegleichung können wiederum genau analog geführt werden.

Nun beweisen wir die in beiden vorangegangenen Sätzen benutzten Hilfssätze.

Lemma 3.3. Sei $\partial \Omega \in C^{2, \alpha}$, dann gilt $K^{*} \in L\left(C^{0, \alpha}(\partial \Omega), C^{1, \alpha}(\partial \Omega)\right)$. 
Beweis. Im dreidimensionalen wurde dies in [29], Theorem 2.8, bewiesen. Der Beweis im $\mathbb{R}^{2}$ ist sehr ähnlich, so daß er hier nur kurz skizziert wird.

Zunächst betrachtet man die Aussage für den entsprechenden Operator bei der Laplacegleichung. Da die Behauptung $K_{0} \in L\left(C^{0, \alpha}(\partial \Omega), C^{1, \alpha}(\partial \Omega)\right)$ bereits bewiesen ist (vgl. [6], Theorem $2.22 \mathrm{im} \mathbb{R}^{3},[57]$, Satz 2.34, im $\mathbb{R}^{2}$ ) reicht es aus, die Aussage für

$$
M \psi:=\left(K_{0}^{*}+K_{0}\right) \psi=\int_{\partial \Omega} \nabla_{y} \Phi_{0}(\cdot, y) \cdot(\nu(y)-\nu(\cdot)) \psi(y) \operatorname{ds}(y)
$$

zu zeigen. Zunächst verwenden wir die Produktregel zur Berechnung der Ableitung $\frac{\mathrm{d}}{\mathrm{ds}} M \psi$ für $\psi \in C^{0, \alpha}(\partial \Omega)$ :

$$
\begin{aligned}
\frac{\mathrm{d}}{\mathrm{ds}} M \psi(x) \\
=\frac{\mathrm{d} \nu}{\mathrm{ds}}(x) \cdot \int_{\partial \Omega}(\psi(x)-\psi(y)) \nabla_{y} \Phi_{0}(x, y) \mathrm{ds}(y)-\frac{\mathrm{d} \nu}{\mathrm{ds}}(x) \cdot \psi(x) \int_{\partial \Omega} \nabla_{y} \Phi_{0}(x, y) \mathrm{ds}(y) \\
\quad+\int_{\partial \Omega}(\psi(y)-\psi(x))(\nu(y)-\nu(x)) \cdot \nabla_{x y} \Phi_{0}(x, y) \cdot \vartheta(x) \mathrm{ds}(y) \\
\quad+\psi(x)\left(\frac{\mathrm{d}}{\mathrm{ds}} K_{0} 1(x)-\nu(x) \cdot \frac{\mathrm{d}}{\mathrm{ds}} \int_{\partial \Omega} \nabla_{y} \Phi_{0}(x, y) \mathrm{ds}(y)\right) \\
=\frac{\mathrm{d} \nu}{\mathrm{ds}}(x) \cdot \int_{\partial \Omega}(\psi(x)-\psi(y)) \nabla_{y} \Phi_{0}(x, y) \mathrm{ds}(y) \\
\quad+\int_{\partial \Omega}(\psi(y)-\psi(x))(\nu(y)-\nu(x)) \cdot \nabla_{x y} \Phi_{0}(x, y) \cdot \vartheta(x) \mathrm{ds}(y) \\
\quad-\psi(x) \frac{\mathrm{d}}{\mathrm{ds}}\left[\nu(x) \cdot\left(K_{0} \nu(x)-S_{0} \frac{\mathrm{d} \vartheta}{\mathrm{ds}}(x)\right)\right] .
\end{aligned}
$$

Hierbei wurde die Gleichung $K_{0} 1=-1 / 2$ (vgl. [57], Seite 28) ausgenutzt. Hieraus ergibt sich $\frac{\mathrm{d}}{\mathrm{ds}} K_{0} 1=0$. Weiterhin wurde die Beziehung

$$
\int_{\partial \Omega} \nabla_{y} \Phi_{0}(x, y) \mathrm{ds}(y)=K_{0} \nu(x)-S_{0} \frac{\mathrm{d} \vartheta}{\mathrm{ds}}(x),
$$

d.h. die Aufspaltung des Gradienten in Tangential- und Normalkomponente benutzt. Danach ist $\frac{\mathrm{d}}{\mathrm{ds}} M \in L\left(C^{0, \alpha}(\partial \Omega), C^{0, \alpha}(\partial \Omega)\right)$ nachzuweisen. Bei dem letzten Term folgt dies aus den Abbildungseigenschaften von $S$ und $K$. Bei den beiden Integralen benutzt man Satz 2.20 in [57]. Nun verwenden wir die Abbildungseigenschaften von $K^{*}-K_{0}^{*}$. Hieraus folgt die Behauptung.

Das nächste Lemma befasst sich nur mit dem zweidimensionalen Fall. Hierzu betrachtet man den vektorwertigen Operator $A$ mit

$$
A \varphi(x):=\int_{\partial \Omega} \nabla_{x} \Phi(x, y) \varphi(y) \odot \vartheta(y) \cdot \nu(x) \mathrm{ds}(y) .
$$

Für diesen Operator gelten folgende Abbildungseigenschaften: 
Lemma 3.4. Sei $\Omega \subset \mathbb{R}^{2}$ ein $C^{m, \alpha}$-Gebiet mit $m \in \mathbb{N}_{\geq 2}$, dann gilt $A \in$ $L\left(C^{m-2, \alpha}(\partial \Omega), C^{m-1, \alpha}\left(\partial \Omega, \mathbb{R}^{2}\right)\right)$.

Beweis. Sei zunächst $m=2, \varphi \in C^{0, \alpha}(\partial \Omega)$ und $a:=\varphi \vartheta$, dann gilt für $x \in \partial \Omega$ :

$$
\begin{aligned}
A \varphi(x)= & \int_{\partial \Omega} \nabla_{x} \Phi(x, y) \odot(a(y)-a(x)) \cdot \nu(x) \mathrm{ds}(y) \\
= & \int_{\partial \Omega} \nabla_{x} \Phi(x, y) \odot(a(y)-a(x)) \cdot(\nu(x)-\nu(y)) \mathrm{ds}(y) \\
& +\int_{\partial \Omega} \nabla_{x} \Phi(x, y) \odot(a(y)-a(x)) \cdot \nu(y) \mathrm{ds}(y) \\
= & : I_{1} \varphi(x)+I_{2} \varphi(x) .
\end{aligned}
$$

Zunächst gilt $I_{1} \in L\left(C^{0, \alpha}(\partial \Omega), C^{1, \alpha}(\partial \Omega)\right)$. Dies kann ähnlich wie im letzten Lemma gezeigt werden (vgl. auch [29], Theorem 2.9 für den analogen Fall im $\mathbb{R}^{3}$ ). Weiterhin ergibt sich:

$$
\begin{aligned}
I_{2} \varphi(x) & =\int_{\partial \Omega} \nabla_{y} \Phi(x, y) \odot \nu(y) \cdot a(x) \mathrm{ds}(y) \\
& =K(\nu \odot \nu \cdot a(x))-S\left(\frac{\mathrm{d} \vartheta}{\mathrm{ds}} \odot \nu \cdot a(x)+\vartheta \odot \frac{\mathrm{d} \nu}{\mathrm{ds}} \cdot a(x)\right)
\end{aligned}
$$

und aus den Abbildungseigenschaften von $S$ und $K$ folgt $I_{2} \in L\left(C^{0, \alpha}(\partial \Omega), C^{1, \alpha}(\partial \Omega)\right)$. Somit ist die Aussage für $m=2$ gezeigt.

Sei nun $m \geq 3, \varphi \in C^{m-2, \alpha}(\partial \Omega)$, dann folgt:

$$
\begin{aligned}
A \varphi(x) & =-\int_{\partial \Omega} \nabla_{y} \Phi(x, y) \odot a(y) \cdot \nu(x) \mathrm{ds}(y) \\
& =\left(-K(\nu \odot a)(x)+2 S\left(\frac{\mathrm{d} \vartheta}{\mathrm{ds}} \odot a\right)(x)+S\left(\frac{\mathrm{d} \varphi}{\mathrm{ds}} \vartheta \odot \vartheta\right)(x)\right) \cdot \nu(x)
\end{aligned}
$$

Unter Beachtung von $(A \varphi) \cdot \vartheta=\left(\frac{\mathrm{d}}{\mathrm{ds}} S(\varphi \vartheta)\right) \cdot \nu$ folgt somit

$$
\begin{aligned}
\frac{\mathrm{d}}{\mathrm{ds}} A \varphi(x)= & \left(2 \frac{\mathrm{d}}{\mathrm{ds}} S\left(\frac{\mathrm{d} \vartheta}{\mathrm{ds}} \odot a\right)(x)-\frac{\mathrm{d}}{\mathrm{ds}} K(\nu \odot a)(x)\right) \cdot \nu(x)+A\left(\odot \vartheta \frac{\mathrm{d} \varphi}{\mathrm{ds}}\right)(x) \\
& +\left(S \frac{\mathrm{d}}{\mathrm{ds}}(\vartheta \odot a)(x)-K(\nu \odot a)(x)\right) \cdot \frac{\mathrm{d} \nu}{\mathrm{ds}}(x)
\end{aligned}
$$

und induktiv mit Hilfe der Abbildungseigenschaften von $S$ und $K$ und der Induktionsvoraussetzung für $A$ die Aussage des Satzes. 
Lemma 3.5. Seien $p>0, x, y, z \in \mathbb{R}^{d}$ mit $x \neq y \neq z$ und $2|x-z| \leq|x-y|$, dann gilt (für eine geeignete Konstante $C>0$ ):

$$
\begin{aligned}
& \left|\frac{x-y}{|x-y|^{p}}-\frac{z-y}{|z-y|^{p}}\right| \leq C \frac{|z-x|}{|x-y|^{p}}, \\
& \left|\frac{1}{|x-y|^{p}}-\frac{1}{|z-y|^{p}}\right| \leq C \frac{|z-x|}{|x-y|^{p+1}} .
\end{aligned}
$$

Beweis. Für $t \in[0,1]$ sei $x(t):=x+t(z-x)$, dann folgt:

$$
|x(t)-y| \geq|x-y|-t|z-x| \geq|x-y|-t / 2|x-y| \geq 1 / 2|x-y|
$$

und somit gilt:

$$
\begin{aligned}
\left|\frac{x-y}{|x-y|^{p}}-\frac{z-y}{|z-y|^{p}}\right| & =\left|\int_{0}^{1} \frac{z-x}{|x(t)-y|^{p}}-p(x(t)-y) \odot \frac{(z-x) \cdot(x(t)-y)}{|x(t)-y|^{p+2}} \mathrm{~d} t\right| \\
& \leq C \frac{|z-x|}{|x-y|^{p}}
\end{aligned}
$$

Die zweite Aussage kann völlig analog bewiesen werden.

Lemma 3.6. Sei $f(x, y):=[(x-y) \cdot \nu(y)] /|x-y|^{2}, g(x, y):=[(x-y) \cdot \vartheta(y)]^{2} /|x-y|^{2}$, $x \neq y \in \mathbb{R}^{d}$.

1. Für $x, y \in \partial \Omega \in C^{1, \alpha}, x \neq y$, gilt $|f(x, y)| \leq C|x-y|^{\alpha-1}$.

2. Für $\partial \Omega \in C^{2}$ sind $f$ und $g$ auf $\partial \Omega \times \partial \Omega$ stetig fortsetzbar.

3. Für $\partial \Omega \in C^{2, \alpha}$ sind $f$ und $g$ auf $\partial \Omega \times \partial \Omega C^{0, \alpha}$-Hölder-stetig fortsetzbar.

Beweis. Man beachte, daß es sich bei allen Behauptungen um lokale Aussagen handelt (vgl. auch Theorem 7.3 in [37]). Zunächst zur ersten Behauptung. Wir zeigen nur den zweidimensionalen Fall. Hier sei $z$ eine Parametrisierung des Randes. $\operatorname{Im} \mathbb{R}^{3}$ können die Behauptungen analog durch lokale Parametrisierungen bewiesen werden. Sei $\tilde{\Gamma} \subset \Gamma$ so klein, daß $D \subset \mathbb{R}$ existiert, so daß $z: D \rightarrow \tilde{\Gamma}$ bijektiv ist. Aus der Taylorschen Formel folgt für $x=z(t), y=z(\tau)$ mit $t, \tau \in D$ :

$$
x-y=z(t)-z(\tau)=(t-\tau) \int_{0}^{1} z^{\prime}(\tau+s(t-\tau)) \mathrm{d} s .
$$

Hieraus folgt $|x-y|^{2} \geq c|t-\tau|^{2}$ (für $\tilde{\Gamma}$ hinreichend klein) und somit (mit $\nu(\tau):=$ $\nu(z(\tau)))$

$$
\begin{aligned}
|f(z(t), z(\tau))| & =\left|\frac{(t-\tau) \int_{0}^{1}\left(z^{\prime}(\tau+s(t-\tau))-z^{\prime}(\tau)\right) \cdot \nu(\tau) \mathrm{d} s}{(t-\tau)^{2} \int_{0}^{1} \int_{0}^{1} z^{\prime}\left(\tau+s_{1}(t-\tau)\right) \cdot z^{\prime}\left(\tau+s_{2}(t-\tau)\right) \mathrm{d} s_{1} \mathrm{~d} s_{2}}\right| \\
& \leq c|t-\tau|^{\alpha-1} \leq C|x-y|^{\alpha-1} .
\end{aligned}
$$


Für die nächste Behauptung wird $z$ bis zur zweiten Ableitung entwickelt:

$$
z(t)-z(\tau)=(t-\tau) z^{\prime}(\tau)+(t-\tau)^{2} \int_{0}^{1}(1-s) z^{\prime \prime}(\tau+s(t-\tau)) \mathrm{d} s
$$

Somit gilt für $t \neq \tau$ :

$$
f(z(t), z(\tau))=\frac{\int_{0}^{1}(1-s) z^{\prime \prime}(\tau+s(t-\tau)) \cdot \nu(\tau) \mathrm{d} s}{\int_{0}^{1} \int_{0}^{1} z^{\prime}\left(\tau+s_{1}(t-\tau)\right) \cdot z^{\prime}\left(\tau+s_{2}(t-\tau)\right) \mathrm{d} s_{1} \mathrm{~d} s_{2}}=: \frac{Z(t, \tau)}{N(t, \tau)}
$$

und $f(z(t), z(t))=\left(z^{\prime \prime}(t) \nu(t)\right) /\left(2\left|z^{\prime}(t)\right|^{2}\right)$. Der Nachweis der stetigen Fortsetzbarkeit von $g$ wird analog geführt.

Nun zur letzten Behauptung. Die Funktion $w: \mathbb{R} \backslash\{0\} \rightarrow \mathbb{R}$ mit $w(t)=1 / t$ ist differenzierbar, so daß aus der Hölder-Stetigkeit von $Z$ und $N$, sowie der strikten Positivität von $N$, die Hölder-Stetigkeit von $f(z(t), z(\tau))=Z(t, \tau) w(N(t, \tau))$ folgt. Wiederum wird der Nachweis für $g$ entsprechend geführt.

Zum Ende dieses Abschnitts über Einfach- und Doppelschichtpotential geben wir eine Variante von Satz 2.18 aus [57] an, da er in den vorangegangenen Beweisen öfters benutzt wurde:

Satz 3.7. Sei $G \subset \mathbb{R}^{2}$ ein kompaktes Gebiet. $K: G \times \partial \Omega \rightarrow \mathbb{C}$ sei stetig für alle $x \in G, y \in \partial \Omega$ mit $x \neq y$. Weiterhin gebe es Konstanten $C>0$ und $\alpha \in] 0,1[$, so daß für $x \in G, y \in \partial \Omega$ mit $x \neq y$ gilt:

$$
|K(x, y)| \leq C|x-y|^{\alpha-1} .
$$

Für $x, z \in G, y \in \partial \Omega$ mit $x \neq y \neq z$ und $2|x-z| \leq|x-y|$ gelte weiterhin:

$$
|K(x, y)-K(z, y)| \leq C|x-z||x-y|^{\alpha-2}
$$

Dann folgt $u \in L\left(C(\partial \Omega), C^{0, \alpha}(G)\right)$ für

$$
u(x):=\int_{\partial \Omega} K(x, y) \varphi(y) \mathrm{ds}(y) .
$$

\subsection{Volumenpotentialoperator}

Für das Gebiet $\Omega$ gelte zunächst $\Omega \subset B_{R}$. Wir betrachten das Volumenpotential $V$, mit

$$
V \varphi(x):=\int_{\Omega} \Phi(x, y) \varphi(y) \mathrm{d} y
$$

über das im folgenden einige Tatsachen zusammengestellt werden. Die Beweise sind zumeist bekannt (vgl. [16], Kapitel 4, für die Laplacegleichung, [20] für den dreidimensionalen Fall und [24] für den zweidimensionalen Fall), so daß sie hier nur knapp dargestellt werden. 
Satz 3.8. 1. Sei $\partial \Omega \in C^{2}$, dann gilt

a) $V \in L\left(C(\bar{\Omega}), C^{1, \alpha}(\bar{\Omega})\right)$.

b) $V: C(\bar{\Omega}) \cap C_{\mathrm{loc}}^{0, \alpha}(\Omega) \rightarrow C^{2}(\Omega) \operatorname{mit}\left(\Delta+\kappa^{2}\right) V \varphi=-\varphi$.

c) $V \in L\left(C^{0, \alpha}(\bar{\Omega}), C^{2, \alpha}(\bar{\Omega})\right)$.

2. Für $m \in \mathbb{N}_{\geq 2}$ und $\partial \Omega \in C^{m, \alpha}$ ist $V \in L\left(C^{m-1, \alpha}(\bar{\Omega}), C^{m+1, \alpha}(\bar{\Omega})\right)$.

Beweis. Sei $\eta \in C^{\infty}(\mathbb{R})$ monoton nicht fallend mit $\eta(t)=0$ für $t \leq 1 / 2$ und $\eta(t)=1$ für $t \geq 1$ (vgl. z.B. [62], Seite 61). Für $l \in \mathbb{N}$ definiert man

$$
V_{l} \varphi(x):=\int_{\Omega} \Phi(x, y) \eta(l|x-y|) \varphi(y) \mathrm{d} y, \quad x \in \bar{\Omega} .
$$

Wir betrachten zunächst den zweidimensionalen Fall. Mit Hilfe von Integration in Polarkoordinaten und der Ungleichung $\left|H_{0}(k r)\right| \leq C(|\ln r|+1), 0<r \leq 2 R$, folgt somit:

$$
\left|V \varphi(x)-V_{l} \varphi(x)\right| \leq C\|\varphi\|_{\infty} \int_{0}^{1 / l}(|\ln r|+1) r \mathrm{~d} r \leq C\|\varphi\|_{\infty}\left(\ln l \frac{1}{l^{2}}+\frac{1}{l^{2}}\right) \stackrel{l \rightarrow \infty}{\longrightarrow} 0 .
$$

Hierbei bezeichnet $C$ eine nur vom Gebiet $\Omega$ und der Dimension $d$ abhängige Konstante, die von Ungleichung zu Ungleichung verschieden sei kann. Sei

$$
V^{(1)} \varphi(x):=\int_{\Omega} \nabla_{x} \Phi(x, y) \varphi(y) \mathrm{d} y, \quad x \in \bar{\Omega}
$$

dann ist $V^{(1)}$ wegen der schwachen Singularität des Kernes wohldefiniert. $V_{l} \varphi$ ist weiterhin offensichtlich differenzierbar mit $\nabla V_{l} \varphi(x)=\int_{\Omega} \nabla_{x}[\Phi(x, y) \eta(l|x-y|)] \varphi(y) \mathrm{d} y$. Wegen $\nabla_{x} \eta(l|x-y|)=0$ für $|x-y| \geq 1 / l$ gilt somit für $x \in \bar{\Omega}$ :

$$
\begin{aligned}
\left|\nabla V_{l} \varphi(x)-V^{(1)} \varphi(x)\right| \leq & \left|\int_{\Omega} \nabla_{x} \Phi(x, y)[\eta(l|x-y|)-1] \varphi(y) \mathrm{d} y\right| \\
& +\left|\int_{\Omega} \Phi(x, y) \nabla_{x} \eta(l|x-y|) \varphi(y) \mathrm{d} y\right| \\
\leq & C\|\varphi\|_{\infty}\left(\int_{0}^{1 / l} \frac{1}{r} r \mathrm{~d} r+l \int_{0}^{1 / l}(|\ln (r)|+1) r \mathrm{~d} r\right) \\
\leq & C \frac{1}{l} \ln l \stackrel{l \rightarrow \infty}{\longrightarrow} 0 .
\end{aligned}
$$

Beides kann ganz analog im dreidimensionalen Fall gezeigt werden. Somit ist $V \varphi$ differenzierbar und es gilt $\nabla V \varphi=V^{(1)} \varphi$. Nun ist noch $\nabla V \in L\left(C(\bar{\Omega}), C^{0, \alpha}\left(\bar{\Omega}, \mathbb{R}^{d}\right)\right)$ 
nachzuweisen. Seien hierzu $x, z \in \bar{\Omega}$ mit $x \neq z, \delta:=|z-x|$, dann folgt aus Lemma 3.5:

$$
\begin{aligned}
|\nabla V \varphi(x)-\nabla V \varphi(z)| \leq & \|\varphi\|_{\infty}\left(\int_{\Omega \cap\{|x-y| \leq 2 \delta\}}\left|\nabla_{x} \Phi(x, y)-\nabla_{x} \Phi(z, y)\right| \mathrm{d} y\right. \\
& \left.+\int_{\Omega \cap\{|x-y| \geq 2 \delta\}}\left|\nabla_{x} \Phi(x, y)-\nabla_{x} \Phi(z, y)\right| \mathrm{d} y\right) \\
\leq & C\|\varphi\|_{\infty}\left(\int_{0}^{3 \delta} \frac{1}{r^{d-1}} r^{d-1} \mathrm{~d} r+|z-x| \int_{\delta}^{2 R} \frac{1}{r^{d}} r^{d-1} \mathrm{~d} r\right) \\
\leq & C\|\varphi\|_{\infty}(3 \delta+|z-x|(\ln 2 R-\ln \delta)) \\
\leq & C\|\varphi\|_{\infty}|z-x|^{\alpha} .
\end{aligned}
$$

In der letzten Ungleichung haben wir die Kompaktheit von $\Omega$ ausgenutzt:

$$
|x-z|=|x-z|^{1-\alpha}|x-z|^{\alpha} \leq(2 R)^{1-\alpha}|x-z|^{\alpha} .
$$

Damit ist 1a) gezeigt.

Zum Nachweis von 1b) sei $\varphi \in C_{\text {loc }}^{0, \alpha}(\Omega)$ und $V_{l}^{(1)} \varphi(x):=\int_{\Omega}\left(\nabla_{x} \Phi(x, y)\right) \eta(l \mid x-$ $y \mid) \varphi(y) \mathrm{d} y$. Wie zu Beginn des Beweises zeigt man $\left\|\nabla V \varphi-V_{l}^{(1)} \varphi\right\|_{\infty, \bar{\Omega}} \stackrel{l \rightarrow \infty}{\longrightarrow} 0$. Offensichtlich gilt $V_{l}^{(1)} \varphi \in C^{1}(\bar{\Omega})$. Weiterhin gilt:

$$
\begin{aligned}
\nabla V_{l}^{(1)} \varphi(x)= & \int_{\Omega} \nabla_{x}\left[\nabla_{x} \Phi(x, y) \eta(l|x-y|)\right](\varphi(y)-\varphi(x)) \mathrm{d} y \\
& -\varphi(x) \int_{\Omega} \nabla_{y}\left[\nabla_{x} \Phi(x, y) \eta(l|x-y|)\right] \mathrm{d} y \\
= & \int_{\Omega} \nabla_{x}\left[\nabla_{x} \Phi(x, y) \eta(l|x-y|)\right](\varphi(y)-\varphi(x)) \mathrm{d} y \\
& -\varphi(x) \int_{\partial \Omega} \nu(y) \odot \nabla_{x} \Phi(x, y) \eta(l|x-y|) \mathrm{ds}(y) .
\end{aligned}
$$

Hierbei wurde im zweiten Schritt der Gauß'sche Integralsatz benutzt. Das erste Integral konvergiert wegen der lokalen Hölder-Stetigkeit von $\varphi$ auf jedem $\Omega^{\prime} \Subset \Omega$ gleichmäßig gegen

$$
I_{1}(x):=\int_{\Omega} \nabla_{x}^{2} \Phi(x, y)(\varphi(y)-\varphi(x)) \mathrm{d} y .
$$

Dies kann ähnlich wie zu Beginn des Beweises gezeigt werden. Weiterhin ist $I_{1}$ lokal Hölder-stetig (vgl. Lemma 3.9). Das zweite Integral konvergiert auf jedem $\Omega^{\prime} \Subset \Omega$ gleichmäßig gegen

$$
I_{2}(x):=\int_{\partial \Omega} \nu(y) \odot \nabla_{x} \Phi(x, y) \mathrm{ds}(y) .
$$

Für den Beweis der Gleichung $\left(\Delta+\kappa^{2}\right) V \varphi=-\varphi$ wird auf [20], Theorem 1.11, und [24], Satz 1.9, verwiesen. 
Für $\varphi \in C^{0, \alpha}(\bar{\Omega})$ folgt $I_{1} \in C^{0, \alpha}(\bar{\Omega})$ aus Lemma 3.9. In Satz 3.2 wurde gezeigt, daß $I_{2}$ hölderstetig auf $\bar{\Omega}$ fortgesetzt werden kann. D.h. es gilt, $\nabla V \varphi \in C(\bar{\Omega}) \cap C^{1}(\Omega)$ und $\nabla^{2} V \varphi$ kann hölderstetig nach $\bar{\Omega}$ fortgesetzt werden. Hieraus folgt $V \varphi \in C^{2, \alpha}(\bar{\Omega})$ und damit ist 1c) gezeigt.

Für $\varphi \in C^{1}(\bar{\Omega})$ folgt mit Hilfe des Gauß'schen Integralsatzes und der Gleichung $\nabla_{x} \Phi=-\nabla_{y} \Phi:$

$$
\begin{aligned}
\nabla V \varphi(x) & =-\int_{\Omega} \nabla_{y} \Phi(x, y) \varphi(y) \mathrm{d} y=-\int_{\Omega} \nabla_{y}(\Phi(x, y) \varphi(y)) \mathrm{d} y+V \nabla \varphi(x) \\
& =V \nabla \varphi(x)-\int_{\partial \Omega} \Phi(x, y)(\varphi \nu)(y) \mathrm{ds}(y)=V \nabla \varphi(x)-\mathcal{S}(\varphi \nu)(x) .
\end{aligned}
$$

Sei nun $m \geq 2, \partial \Omega \in C^{m, \alpha}$ und $\varphi \in C^{m-1, \alpha}(\bar{\Omega})$ und als Induktionsvoraussetzung gilt $V \in L\left(C^{m-2, \alpha}(\bar{\Omega}), C^{m, \alpha}(\bar{\Omega})\right)$. Dann folgt aus (3.20) und der Abbildungseigenschaft des Operators $\mathcal{S}$ die Regularität $\nabla V \varphi \in C^{m, \alpha}(\bar{\Omega})$ und somit $V \varphi \in C^{m+1, \alpha}(\bar{\Omega})$.

Lemma 3.9. Sei $\Omega$ ein $C^{2}$-Gebiet.

1. Das Integral $\int_{\Omega} \nabla_{x}^{2} \Phi(x, y) \mathrm{d} y$ existiert als Cauchy-Hauptwert für alle $x \in \bar{\Omega}$ und es existiert ein $M>0$ mit $\left\|\int_{\Omega} \nabla_{x}^{2} \Phi(\cdot, y) \mathrm{d} y\right\|_{\infty, \bar{\Omega}} \leq M$.

2. Es gilt $A \in L\left(C^{0, \alpha}(\bar{\Omega}), C^{0, \alpha}\left(\bar{\Omega}, \mathbb{R}^{2 \times 2}\right)\right)$ für $A \varphi(x):=\int_{\Omega} \nabla_{x}^{2} \Phi(x, y)(\varphi(y)-$ $\varphi(x)) \mathrm{d} y$.

Beweis. Sei $x \in \Omega$ und $r>0$ hinreichend klein, dann folgt aus dem Gauß'schen Integralsatz:

$$
\begin{aligned}
\int_{\Omega \backslash B_{r}(x)} \nabla_{x}^{2} \Phi(x, y) \mathrm{d} y & =-\int_{\Omega \backslash B_{r}(x)} \nabla_{y} \nabla_{x} \Phi(x, y) \mathrm{d} y \\
& =-\int_{\partial \Omega} \nu(y) \odot \nabla_{x} \Phi(x, y) \mathrm{ds}(y)+\int_{\partial B_{r}(x)} \nu(y) \odot \nabla_{x} \Phi(x, y) \mathrm{ds}(y) \\
& =: I_{1}(x)+I_{2}(x) .
\end{aligned}
$$

Das Integral $I_{1}$ entspricht der Ableitung des Einfachschichtpotentials $\mathcal{S}$ mit differenzierbarer Dichte und stellt somit eine in ganz $\bar{\Omega}$ hölderstetige Funktion dar. Das Integral $I_{2}$ kann unter Verwendung von Polarkoordinaten (exemplarisch wird der zweidimensionale Fall gezeigt: $y=x+(r \cos t, r \sin t)$; im $\mathbb{R}^{3}$ führen analoge Berechnungen zum Erfolg) explizit berechnet werden:

$$
\begin{aligned}
I_{2}(x) & =\frac{i \kappa}{4} \int_{0}^{2 \pi}\left(\begin{array}{c}
\cos t \\
\sin t
\end{array}\right) \odot\left(\begin{array}{c}
r \cos t \\
r \sin t
\end{array}\right) \frac{H_{1}(\kappa r)}{r} r \mathrm{~d} t \\
& \stackrel{r \rightarrow 0}{\longrightarrow} \frac{1}{2 \pi} \int_{0}^{2 \pi}\left(\begin{array}{c}
\cos t \\
\sin t
\end{array}\right) \odot\left(\begin{array}{c}
\cos t \\
\sin t
\end{array}\right) \mathrm{d} t=0 .
\end{aligned}
$$


Sei nun $x \in \partial \Omega, \Gamma_{r}:=\{y \in \partial \Omega:|y-x| \geq r\}$ und $\tilde{\Gamma}_{r}:=\partial B_{r}(x) \cap \Omega$, dann gilt:

$$
\begin{aligned}
\int_{\Omega \backslash B_{r}(x)} \nabla_{x}^{2} \Phi(x, y) \mathrm{d} y & =-\int_{\Gamma_{r}} \nu(y) \odot \nabla_{x} \Phi(x, y) \mathrm{ds}(y)+\int_{\tilde{\Gamma}_{r}} \nu(y) \odot \nabla_{x} \Phi(x, y) \mathrm{ds}(y) \\
& =: I_{1}(x)+I_{2}(x) .
\end{aligned}
$$

Zunächst gilt $\lim _{r \rightarrow 0} I_{1}=-\nabla \mathcal{S} \nu$ (vgl. die Beweise von [6], Theorem 2.17, im $\mathbb{R}^{3}$ und [57], Satz 2.27, im $\mathbb{R}^{2}$ ). $I_{2}$ kann genau wie oben berechnet werden und es ergibt sich unter Verwendung von $\partial \Omega \in C^{2}$ (vgl. auch Lemma 2.2 in [45]):

$$
\lim _{r \rightarrow 0} I_{2}(x)=\frac{1}{2 \pi} \int_{0}^{\pi}\left(\begin{array}{c}
\cos t \\
\sin t
\end{array}\right) \odot\left(\begin{array}{c}
\cos t \\
\sin t
\end{array}\right) \mathrm{d} t .
$$

Damit ist die erste Behauptung gezeigt.

Seien nun $x, z \in \bar{\Omega}$ mit $\delta:=|x-z|>0$, dann gilt unter Beachtung der ersten Behauptung:

$$
\begin{aligned}
|A(x)-A(z)| \leq & \left|\int_{\Omega \cap B_{2 \delta}(x)} \nabla_{x}^{2} \Phi(x, y)(\varphi(y)-\varphi(x)) \mathrm{d} y\right| \\
& +\left|\int_{\Omega \cap B_{2 \delta}(x)} \nabla_{z}^{2} \Phi(z, y)(\varphi(y)-\varphi(z)) \mathrm{d} y\right| \\
& +\left|\int_{\Omega \backslash B_{2 \delta}(x)}\left(\nabla_{x}^{2} \Phi(x, y)-\nabla_{z}^{2} \Phi(z, y)\right)(\varphi(y)-\varphi(z)) \mathrm{d} y\right| \\
& +\left.\|\varphi\|_{\alpha}|x-z|^{\alpha}\left|\int_{\Omega \backslash B_{2 \delta}(x)} \nabla_{x}^{2} \Phi(x, y) \mathrm{d} y\right|\right|_{\Omega-d} \mathrm{~d} y+C\|\varphi\|_{\alpha} \int_{\Omega \cap B_{z}(3 \delta)}|z-y|^{\alpha-d} \mathrm{~d} y \\
\leq & C\|\varphi\|_{\alpha} \int_{\Omega \cap B_{2 \delta}(x)}|x-y|^{\alpha-1}|x-y|^{\alpha-d-1} \mathrm{~d} y+C \|_{\alpha}|x-z|^{\alpha} \\
& +C|x-z|\|\varphi\|_{\alpha} \int_{\Omega \backslash B_{2 \delta}(x)}|x-z|^{\alpha} .
\end{aligned}
$$

Für die vorletzte Ungleichung haben wir hierbei die Dreiecksungleichung verwendet. Für $y \in \Omega \backslash B_{2 \delta}(x)$ gilt

$$
\begin{aligned}
|\varphi(y)-\varphi(z)| & \leq|\varphi(y)-\varphi(x)|+|\varphi(x)-\varphi(z)| \\
& \leq C\left(|y-x|^{\alpha}+|x-z|^{\alpha}\right) \leq 2 C|y-x|^{\alpha} .
\end{aligned}
$$

Zum Ende dieses Abschnitts betrachten wir noch den Fall, daß $\Omega$ unbeschränkt ist. Um die Existenz des Volumenpotentials zu garantieren, setzen wir nun allerdings voraus, daß die Dichte $\varphi$ in $B_{R, e}$ verschwindet:

$$
\begin{aligned}
C_{0}(\bar{\Omega}) & :=\left\{\varphi \in C(\bar{\Omega}):\left.\quad \varphi\right|_{B_{R, e}}=0\right\} \\
C_{0}^{m, \alpha}(\bar{\Omega}) & :=C^{m, \alpha}(\bar{\Omega}) \cap C_{0}(\bar{\Omega}) .
\end{aligned}
$$


Korollar 3.10. 1. Sei $\partial \Omega \in C^{2}$, dann gilt

a) $V \in L\left(C_{0}(\bar{\Omega}), C^{1, \alpha}(\bar{\Omega})\right)$.

b) $V: C_{0}(\bar{\Omega}) \cap C_{\mathrm{loc}}^{0, \alpha}(\Omega) \rightarrow C^{2}(\Omega) \operatorname{mit}\left(\Delta+\kappa^{2}\right) V \varphi=-\varphi$.

c) $V \in L\left(C_{0}^{0, \alpha}(\bar{\Omega}), C^{2, \alpha}(\bar{\Omega})\right)$.

2. Für $m \in \mathbb{N}_{\geq 2}$ und $\partial \Omega \in C^{m, \alpha}$ ist $V \in L\left(C_{0}^{m-1, \alpha}(\bar{\Omega}), C^{m+1, \alpha}(\bar{\Omega})\right)$. 


\section{Randwertprobleme}

Ziel dieses Abschnitts ist der Nachweis der Lösbarkeit einiger Randwertprobleme zur inhomogenen Helmholtzgleichung. Der Existenzbeweis kann bei allen betrachteten Problemen in zwei Teilprobleme aufgespalten werden. Zum einen wird die homogene Differentialgleichung mit inhomogenen Randwerten betrachtet. Die Beweisideen sind bei diesem Teil den Arbeiten von Kirsch ([29], Paragraph 3) und Colton/Kress ([7], Kapitel 3.2) entnommen. Zur Lösung der inhomogenen Differentialgleichung wird ein Volumenpotential verwendet. Eine interessante Variante, bei der auch noch ortsabhängige Wellenzahlen in einem kompakten Teilbereich vom betrachteten Gebiet zugelassen sind, findet man in Leis ([44], Satz 7.6).

Es werden zunächst einige Räume eingeführt. Es gelte $m \in \mathbb{N}_{\geq 2}, \partial \Omega \in C^{m, \alpha}$ und $j \in \mathbb{N}_{\geq 0}$. Das Gebiet $\Omega$ ist dabei weiterhin von der in Kapitel 2 beschriebenen Form. Insbesondere kann es sowohl beschränkt, als auch unbeschränkt sein und für unbeschränktes $\Omega$ fordern wir, daß $B_{R, e}$ echt in $\Omega$ enthalten ist. Dies wird bei der Definition der folgenden Funktionenräume verwendet.

$$
\begin{aligned}
& \hat{C}^{m, \alpha}(\bar{\Omega}):= \begin{cases}C^{m, \alpha}(\bar{\Omega}) & \text { falls } \Omega \text { beschränkt ist }, \\
\left\{u \in C^{m, \alpha}(\bar{\Omega}):\left.\left(\Delta+\kappa^{2}\right) u\right|_{B_{R, e}}=0,\right. & \\
u \text { erfüllt die SAB }\} & \text { sonst. }\end{cases} \\
& C_{0}^{j, \alpha}(\bar{\Omega}):= \begin{cases}C^{j, \alpha}(\bar{\Omega}) & \text { falls } \Omega \text { beschränkt } \\
\left\{u \in C^{j, \alpha}(\bar{\Omega}):\left.u\right|_{B_{R, e}}=0\right\} & \text { sonst. }\end{cases}
\end{aligned}
$$

Diese Räume benötigen wir für Lösungen bei der inhomogenen Helmholtzgleichung. Sei $\Omega$ unbeschränkt und $\varphi \in C_{0}^{m-2, \alpha}(\bar{\Omega})$ und $\psi:=-V \varphi$. Aus Korollar 3.10 folgt dann $\psi \in \hat{C}^{m, \alpha}(\bar{\Omega}) \operatorname{mit}\left(\Delta+\kappa^{2}\right) \psi=\varphi$.

Lemma 4.1. $\hat{C}^{m, \alpha}(\bar{\Omega})$ und $C_{0}^{j, \alpha}(\bar{\Omega})$ sind Banachräume.

Beweis. Für $C_{0}^{j, \alpha}(\bar{\Omega})$ ist die Aussage klar, ebenso wie für $\hat{C}^{m, \alpha}(\bar{\Omega})$ bei beschränktem $\Omega$. Sei also $\Omega$ unbeschränkt und $\left(u_{n}\right)_{n \in \mathbb{N}} \subset \hat{C}^{m, \alpha}(\bar{\Omega})$ eine Cauchy-Folge. Wegen der Vollständigkeit von $C^{m, \alpha}(\bar{\Omega})$ existiert ein $u \in C^{m, \alpha}(\bar{\Omega})$, gegen das die Folge konvergiert. Aus dem Greenschen Darstellungssatz (vgl. [6], Theorem 3.3) folgt

$$
u_{n}=\mathcal{K}_{R} u_{n}-\mathcal{S}_{R} \frac{\partial u_{n}}{\partial \nu}
$$

im Äußeren von $B_{R}$, wobei $\mathcal{S}_{R}$ und $\mathcal{K}_{R}$ den Einfach- und den Doppelschichtpotentialoperator bzgl. des Randes $\partial B_{R}$ bezeichnen. Aus der Stetigkeit von $\mathcal{S}_{R}$ und $\mathcal{K}_{R}$ (vgl. 
Satz 3.1) folgt die Gleichung

$$
u=\mathcal{K}_{R} u-\mathcal{S}_{R} \frac{\partial u}{\partial \nu}
$$

und somit $u \in \hat{C}^{m, \alpha}(\bar{\Omega})$.

Nun betrachten wir das Dirichlet-, das Neumann-- und das Robinproblem. Hierzu definieren wir Operatoren

$$
\begin{array}{ll}
\mathcal{R}_{D} \in L(C(\bar{\Omega}), C(\partial \Omega)), & \mathcal{R}_{D} u=u, \\
\mathcal{R}_{N} \in L\left(C^{1}(\bar{\Omega}), C(\partial \Omega)\right), & \mathcal{R}_{N} u=\frac{\partial u}{\partial \nu}, \\
\mathcal{R}_{R} \in L\left(C^{1}(\bar{\Omega}), C(\partial \Omega)\right), & \mathcal{R}_{R} u=\frac{\partial u}{\partial \nu}+i \eta u=\mathcal{R}_{N} u+i \eta \mathcal{R}_{D} u .
\end{array}
$$

Hierbei sei $\eta \in \mathbb{R} \backslash\{0\}$ eine Konstante mit $\eta \operatorname{Re}(\kappa) \leq 0$, falls $\Omega$ beschränkt ist, sonst $\eta \operatorname{Re}(\kappa) \geq 0$. Weiterhin betrachten wir den Differentialoperator

$$
\mathcal{L} \in L\left(C^{2}(\bar{\Omega}), C(\bar{\Omega})\right), \quad \mathcal{L} u=\left(\Delta+\kappa^{2}\right) u .
$$

Man beachte, daß $\mathcal{L} u \in C_{0}^{m-2, \alpha}(\bar{\Omega})$ für $u \in \hat{C}^{m, \alpha}(\bar{\Omega})$ gilt.

\subsection{Robinproblem}

Satz 4.2. Für $g \in C_{0}^{m-2, \alpha}(\bar{\Omega})$ und $f \in C^{m-1, \alpha}(\partial \Omega)$ existiert genau ein $u \in \hat{C}^{m, \alpha}(\bar{\Omega})$ mit

$$
\left(\mathcal{L}, \mathcal{R}_{R}\right) u=(g, f)
$$

Für den Operator $\mathcal{D}_{R}:(g, f) \mapsto u$ gilt $\mathcal{D}_{R} \in L\left(C_{0}^{m-2, \alpha}(\bar{\Omega}) \times C^{m-1, \alpha}(\partial \Omega), \hat{C}^{m, \alpha}(\bar{\Omega})\right)$. Weiterhin ist $\mathcal{D}_{R}$ invertierbar mit $\mathcal{D}_{R}^{-1}=\left(\mathcal{L}, \mathcal{R}_{R}\right)$.

Beweis. Zunächst wollen wir zeigen, daß zu $f \in C^{m-1, \alpha}(\partial \Omega)$ genau ein $u \in \hat{C}^{m, \alpha}(\bar{\Omega})$ mit $\mathcal{L} u=0$ und $\mathcal{R}_{R} u=f$ existiert. Wir beginnen mit der Eindeutigkeit, d.h. sei $u \in \hat{C}^{m, \alpha}(\bar{\Omega})$ mit $\mathcal{L} u=0$ und $\mathcal{R}_{R} u=0$. Für unbeschränktes $\Omega$ ergibt sich aus $\partial \bar{u} / \partial \nu=$ $\left.i \eta \bar{u}\right|_{\partial \Omega}$ die Gleichung

$$
\operatorname{Im}\left(\kappa \int_{\partial \Omega} u \frac{\overline{\partial u}}{\partial \nu} \mathrm{d} s\right)=\eta \operatorname{Re}(\kappa) \int_{\partial \Omega}|u|^{2} \mathrm{ds} \geq 0
$$

und somit folgt $u=0$ (vgl. [6], Theorem 3.12, im $\mathbb{R}^{3}$ und [57], Korollar 2.7. für den zweidimensionalen Fall). Für beschränktes $\Omega$ folgt aus dem ersten Greenschen Satz und $\eta u=i \partial u / \partial \nu$ die Gleichung

$$
\begin{aligned}
\eta \int_{\partial \Omega}|u|^{2} \mathrm{~d} s & =\operatorname{Re}\left(\eta \int_{\partial \Omega}|u|^{2} \mathrm{~d} s\right)=\operatorname{Re}\left(i \int_{\partial \Omega} \bar{u} \frac{\partial u}{\partial \nu} \mathrm{d} s\right) \\
& =-\operatorname{Im}\left(\int_{\partial \Omega} \bar{u} \frac{\partial u}{\partial \nu} \mathrm{d} s\right)=2 \operatorname{Im}(\kappa) \operatorname{Re}(\kappa) \int_{\Omega}|u|^{2} \mathrm{~d} x
\end{aligned}
$$


Nun gilt entweder $\operatorname{Re}(\kappa)=0$ und daher $\int_{\partial \Omega}|u|^{2} \mathrm{~d} s=0$, oder

$$
\underbrace{\eta \operatorname{Re}(\kappa)}_{<0} \int_{\partial \Omega}|u|^{2} \mathrm{~d} s=2 \operatorname{Im}(\kappa) \operatorname{Re}(\kappa)^{2} \int_{\Omega}|u|^{2} \mathrm{~d} x \geq 0,
$$

d.h. ebenfalls $\int_{\partial \Omega}|u|^{2} \mathrm{~d} s=0$. In beiden Fällen folgt somit $\mathcal{R}_{D} u=\mathcal{R}_{N} u=0$ und daher $u=0$ aus dem Greenschen Darstellungssatz.

Nun zur Existenz eines $u \in \hat{C}^{m, \alpha}(\bar{\Omega})$ mit $\mathcal{L} u=0$ und $\mathcal{R}_{R} u=f$. Sei $\Omega$ unbeschränkt und $u:=(\mathcal{S}+i \eta \mathcal{K}) \varphi, \varphi \in C^{m, \alpha}(\partial \Omega)$, dann gilt $\mathcal{R}_{R} u=\left(A-1 / 2\left(1+\eta^{2}\right) I\right) \varphi$, wobei der Operator

$$
A:=K^{*}+i \eta T+i \eta S-\eta^{2} K
$$

beschränkt von $C^{m, \alpha}(\partial \Omega)$ nach $C^{m-1, \alpha}(\partial \Omega)$ abbildet. Wir wollen zeigen, daß $A-$ $1 / 2\left(1+\eta^{2}\right) I \in L\left(C^{m, \alpha}(\partial \Omega), C^{m-1, \alpha}(\partial \Omega)\right)$ beschränkt invertierbar ist. Sei $\tilde{\kappa}$ eine Wellenzahl, die kein innerer Dirichlet-Eigenwert in $\Omega_{e}$ ist (vgl. Definition 4.3), dann ist $S_{\tilde{\kappa}} \in L\left(C^{m-1, \alpha}(\partial \Omega), C^{m, \alpha}(\partial \Omega)\right)$ beschränkt invertierbar (vgl. [6], Theorem 3.30) und somit ist die Gleichung

$$
\left(A-\frac{1}{2}\left(1+\eta^{2}\right) I\right) \varphi=f
$$

äquivalent $\mathrm{zu}$

$$
S_{\tilde{\kappa}}\left(A-\frac{1}{2}\left(1+\eta^{2}\right) I\right) \varphi=S_{\tilde{\kappa}} f
$$

Nun gilt $S_{\tilde{\kappa}} T_{\tilde{\kappa}}=K_{\tilde{\kappa}}^{2}-I$ (vgl. [7], Gleichung (3.12)) und daher

$$
\begin{aligned}
S_{\tilde{\kappa}}\left(A-\frac{1}{2}\left(1+\eta^{2}\right) I\right)= & S_{\tilde{\kappa}}\left(K^{*}+i \eta\left(T-T_{\tilde{\kappa}}\right)+i \eta S-\eta^{2} K-\frac{1}{2}\left(1+\eta^{2}\right) I\right) \\
& +i \eta\left(K_{\tilde{\kappa}}^{2}-I\right)=: \tilde{A}-i \eta I .
\end{aligned}
$$

Hierbei ist der Operator $\tilde{A}$ infolge von Satz 3.2 in $C^{m, \alpha}(\partial \Omega)$ kompakt und somit reicht es aus, die Injektivität von $A-\frac{1}{2}\left(1+\eta^{2}\right) I$ nachzuweisen. Sei also $\varphi \in N\left(A-\frac{1}{2}\left(1+\eta^{2}\right) I\right)$, dann folgt für $u:=(\mathcal{S}+i \eta \mathcal{K}) \varphi$ die Gleichung $u=0$ in $\bar{\Omega}$. Aus den Sprungbeziehungen ergibt sich dann $-u_{-}=i \eta \varphi$ und $\frac{\partial u_{-}}{\partial \nu}=\varphi$. Eine Anwendung des ersten Greenschen Satzes liefert

$$
\begin{aligned}
\eta \int_{\partial \Omega}|\varphi|^{2} \mathrm{~d} s & =\operatorname{Re}\left(\eta \int_{\partial \Omega} \bar{\varphi} \varphi \mathrm{d} s\right) \\
& =\operatorname{Re}\left(-i \int_{\partial \Omega} \overline{u_{-}} \frac{\partial u_{-}}{\partial \nu} \mathrm{d} s\right)=-2 \operatorname{Im}(\kappa) \operatorname{Re}(\kappa) \int_{\mathbb{R}^{d} \backslash \Omega}|u|^{2} \mathrm{~d} x
\end{aligned}
$$

Durch gleiche Argumentation wie beim Eindeutigkeitsnachweis beim beschränkten Gebiet erhält man $\varphi=0$.

Für ein beschränktes Gebiet $\Omega$ wählt man $u:=(\mathcal{S}-i \gamma \eta \mathcal{K}) \varphi, \varphi \in C^{m, \alpha}(\partial \Omega)$ und $\gamma>0$ mit $1-\gamma \eta^{2} \neq 0$ und argumentiert wie für ein unbeschränktes Gebiet. 
Daher haben wir unter Berücksichtigung von Satz 3.1 die Eindeutigkeit und Existenz eines Operators $\mathcal{D}_{R}^{h} \in L\left(C^{m-1, \alpha}(\partial \Omega), \hat{C}^{m, \alpha}(\bar{\Omega})\right)$ mit $\mathcal{L D}_{R}^{h}=0$ und $\mathcal{R}_{R} \mathcal{D}_{R}^{h}=I$ nachgewiesen. Für den Operator

$$
\mathcal{D}_{R}(g, f):=\mathcal{D}_{R}^{h} f+\mathcal{D}_{R}^{h} \mathcal{R}_{R} V g-V g
$$

gilt $\left(\mathcal{L}, \mathcal{R}_{R}\right) \mathcal{D}_{R}=I$. Sei nun $u \in \hat{C}^{m, \alpha}(\bar{\Omega})$ beliebig und $v:=\mathcal{D}_{R}\left(\mathcal{L} u, \mathcal{R}_{R} u\right)$, dann folgt $\mathcal{L}(u-v)=0$ und $\mathcal{R}_{R}(u-v)=0$ und wegen der Eindeutigkeit des Problems $u=v$, d.h. $\mathcal{D}_{R}=\left(\mathcal{L}, \mathcal{R}_{R}\right)^{-1}$.

\subsection{Dirichletproblem}

Ziel dieses Abschnitts ist es, die Existenz und Eindeutigkeit einer Lösung eines inhomogenen Dirichletproblems zur Helmholtzgleichung zu zeigen. Die Konstante $\kappa^{2}$ sei kein Dirichleteigenwert, falls $\Omega$ beschränkt ist, wobei wir folgende Definition für Dirichlet(Neumann-)Eigenwerte verwenden:

Definition 4.3. Sei $\Omega$ ein beschränktes $C^{2}$-Gebiet und $\lambda \in \mathbb{C}$. Funktionen $u_{D} \in$ $C^{2}(\Omega) \cap C(\bar{\Omega})$ und $u_{N} \in C^{2}(\Omega) \cap C^{1}(\bar{\Omega})$ mit $(\Delta+\lambda) u_{j}=0$ in $\Omega, \int_{\Omega}\left|u_{j}\right|^{2} d x=1$ und $\mathcal{R}_{j} u_{j}=0$ für $j=D, N$ heißen (Dirichlet)-Eigenfunktion bzw. (Neumann)Eigenfunktion des Operators $\Delta$ zum Eigenwert $\lambda$.

Satz 4.4. Für $g \in C_{0}^{m-2, \alpha}(\bar{\Omega})$ und $f \in C^{m, \alpha}(\partial \Omega)$ existiert genau ein $u \in \hat{C}^{m, \alpha}(\bar{\Omega})$ mit

$$
\left(\mathcal{L}, \mathcal{R}_{D}\right) u=(g, f)
$$

Für den Operator $\mathcal{D}_{D}:(g, f) \mapsto u$ gilt $\mathcal{D}_{D} \in L\left(C_{0}^{m-2, \alpha}(\bar{\Omega}) \times C^{m, \alpha}(\partial \Omega), \hat{C}^{m, \alpha}(\bar{\Omega})\right)$. Weiterhin ist $\mathcal{D}_{D}$ invertierbar mit $\mathcal{D}_{D}^{-1}=\left(\mathcal{L}, \mathcal{R}_{D}\right)$.

Beweis. Wie beim Robinproblem wird zunächst die Existenz eines Operators $\mathcal{D}_{D}^{h} \in$ $L\left(C^{m, \alpha}(\partial \Omega), \hat{C}^{m, \alpha}(\bar{\Omega})\right)$ mit $\mathcal{L D}_{D}^{h}=0$ und $\mathcal{R}_{D} \mathcal{D}_{D}^{h}=I$ nachgewiesen. Die Eindeutigkeit dieses Operators gilt für beschränktes $\Omega$ nach Voraussetzung (da $\kappa^{2}$ kein innerer Dirichleteigenwert ist), für unbeschränktes $\Omega$ folgt sie aus der Ungleichung (4.1), die bei homogenen Dirichletrandwerten trivialerweise gilt. Für einen Eindeutigkeitsbeweis beim Dirichlet-Problem, der die Existenz der Normalableitung nicht voraussetzt, verweisen wir auf Theorem 3.7 in [7].

Die Existenz einer Lösung wird wie beim Robinproblem behandelt (vgl. hierzu auch Theorem 3.9 in [7]).

\subsection{Neumannproblem}

Sei $\kappa^{2}$ kein Neumanneigenwert, falls $\Omega$ beschränkt ist. 
Satz 4.5. Für $g \in C_{0}^{m-2, \alpha}(\bar{\Omega})$ und $f \in C^{m-1, \alpha}(\partial \Omega)$ existiert genau ein $u \in \hat{C}^{m, \alpha}(\bar{\Omega})$ mit

$$
\left(\mathcal{L}, \mathcal{R}_{N}\right) u=(g, f)
$$

Für den Operator $\mathcal{D}_{N}:(g, f) \mapsto u$ gilt $\mathcal{D}_{N} \in L\left(C_{0}^{m-2, \alpha}(\bar{\Omega}) \times C^{m-1, \alpha}(\partial \Omega), \hat{C}^{m, \alpha}(\bar{\Omega})\right)$. Weiterhin ist $\mathcal{D}_{N}$ invertierbar mit $\mathcal{D}_{N}^{-1}=\left(\mathcal{L}, \mathcal{R}_{N}\right)$.

Beweis. Wie beim Robinproblem wird auch hier zunächst die Existenz eines Operators $\mathcal{D}_{N}^{h} \in L\left(C^{m-1, \alpha}(\partial \Omega), C^{m, \alpha}(\bar{\Omega})\right)$ mit $\mathcal{L D}_{N}^{h}=0$ und $\mathcal{R}_{D} \mathcal{D}_{N}^{h}=I$ nachgewiesen. Die Eindeutigkeit gilt für beschränktes $\Omega$ nach Voraussetzung, für unbeschränktes $\Omega$ verwenden wir die Ungleichung (4.1). Der Existenznachweis erfolgt dann wie beim Robinproblem. 


\section{Transmissionsproblem}

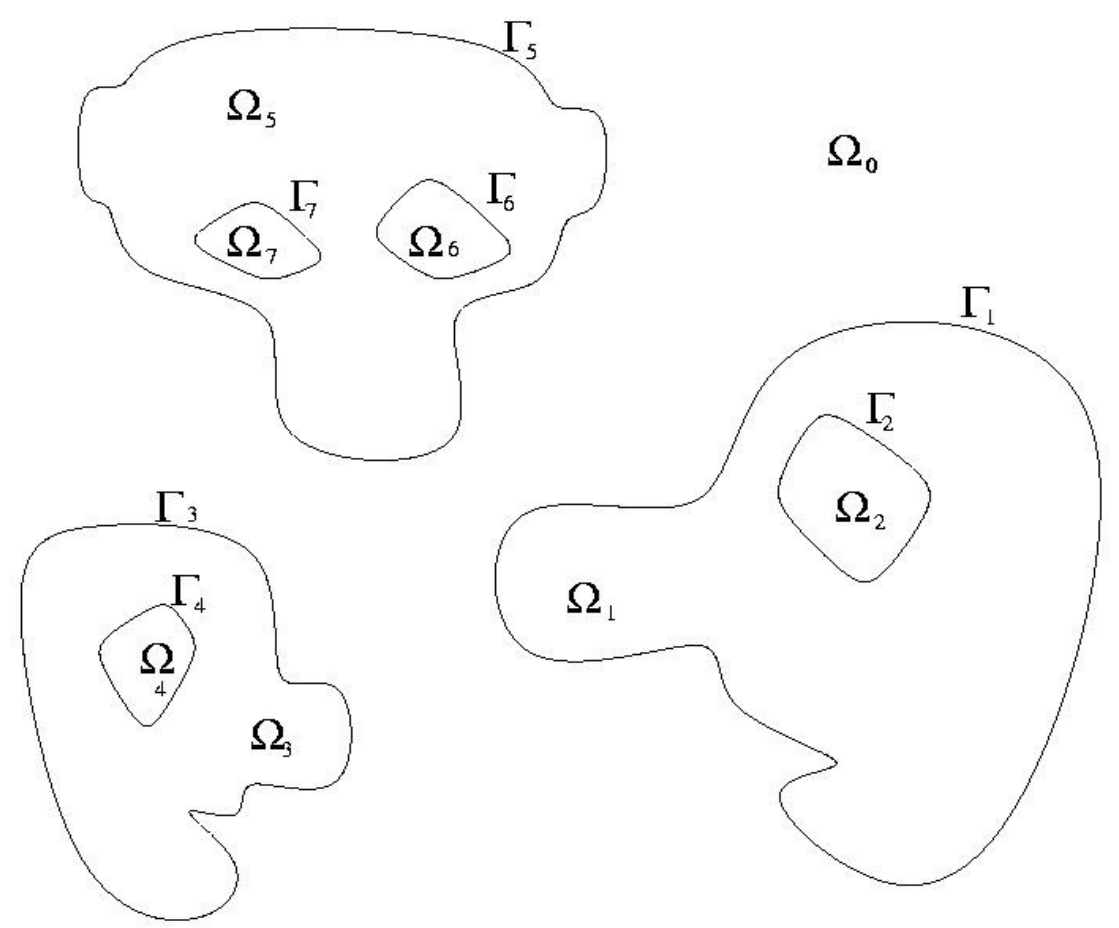

Abbildung 5.1: Beispielgeometrie

Gegeben seien paarweise disjunkte Gebiete (d.h. offene und zusammenhängende Teilmengen des $\mathbb{R}^{d}$ )

$$
\Omega_{0}, \ldots, \Omega_{L}, \quad L \in \mathbb{N}, \text { mit } \Omega:=\bigcup_{j=0}^{L} \Omega_{j} \text { und } \bar{\Omega}=\mathbb{R}^{d} .
$$

$\Omega_{0}$ sei unbeschränkt, die anderen Gebiete beschränkt. $R>0$ sei so groß gewählt, daß $B_{R, e} \Subset \Omega_{0}$ gilt. Weiterhin setzen wir die Existenz beschränkter, regulärer, zusammenhängender Ränder

$$
\Gamma_{1}, \ldots, \Gamma_{L} \operatorname{mit} \Gamma:=\bigcup_{j=1}^{L} \Gamma_{j}=\bigcup_{j=0}^{L} \partial \Omega_{j}
$$


voraus mit $\Gamma \in C^{m, \alpha}, m \in \mathbb{N}_{\geq 2}$ und $\left.\alpha \in\right] 0,1[$.

Wir fordern, daß alle Ränder $\Gamma_{j}$ den $\mathbb{R}^{d}$ in ein beschränktes Innengebiet $\Omega_{j, i}$ und ein unbeschränktes Außengebiet $\Omega_{j, a}$ unterteilen und es gelte stets $\Omega_{j} \subset \Omega_{j, i}, \partial \Omega_{j} \supset \Gamma_{j}$. Zu jedem Gebiet $\Omega_{j}, j=1, \ldots, L$, existiert genau ein Gebiet $\Omega_{k}, k=0, \ldots, L$, mit $\Omega_{k} \subset$ $\Omega_{j, a}$ und $\partial \Omega_{k} \cap \partial \Omega_{j}=\Gamma_{j}$ (in der Abbildung 5.1 z.B. $\Omega_{5} \subset \Omega_{7, a}$ und $\partial \Omega_{5} \cap \partial \Omega_{7}=\Gamma_{7}$ ). Hierzu definieren wir eine Funktion

$$
v:\{1, \ldots, L\} \rightarrow\{0, \ldots, L\} \text { mit } v(j)=k,
$$

mit $k$ wie eben definiert. In der Abbildung 5.1 gilt z.B. $v(7)=5$. Ebenso betrachten wir eine Abbildung

$$
n:\{0, \ldots, L\} \rightarrow \mathcal{P}(\{1, \ldots, L\}) \text { mit } n(k):=\{j \in\{1, \ldots, L\}: v(j)=k\} .
$$

Hierbei ist $\mathcal{P}(\{1, \ldots, L\})$ die Potenzmenge von $\{1, \ldots, L\}$. In der Abbildung 5.1 gilt z.B. $n(0)=\{1,3,5\}$. Bei der Implementierung von verschiedenen Geometrien bietet sich eine Baumstruktur als Speicherungsmethode an (vgl. die Abbildung 5.2). Hier beschreibt die Funktion $v$ gerade den Vorgänger eines Knotens und die Funktion $n$ die nachfolgenden Knoten. Wir setzen stets $v(j)<j$ voraus. Dies ist zwar nicht unbedingt erforderlich, vereinfacht aber die Implementierung, wie sich noch zeigen wird.

Für die Richtung des Normalenvek-

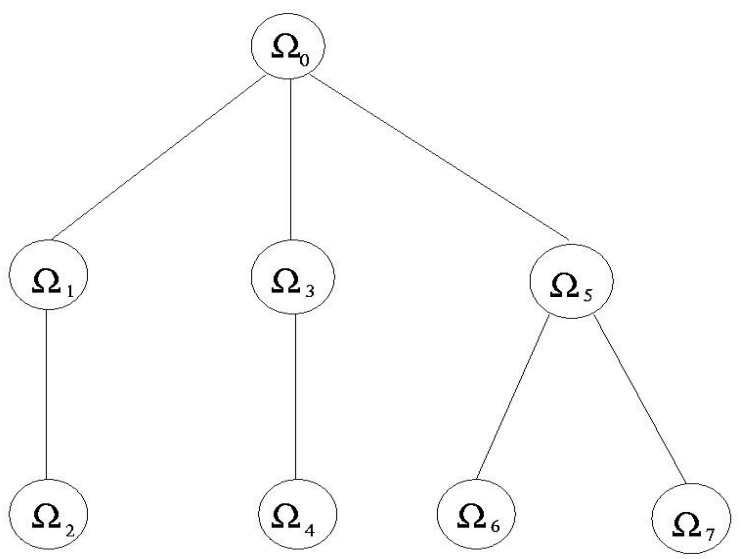
tors $\nu$ führen wir folgende Konvention ein: Wird er bzgl. eines Randes $\partial \Omega_{j}$ betrachtet, so zeige er stets nach $\mathbb{R}^{d} \backslash \overline{\Omega_{j}}$ (außer bei $\partial \Omega_{0}$; hier sei die Normale in Richtung $\Omega_{0}$ orientiert), bzgl. eines Randes $\Gamma_{j}$ zeige er nach $\Omega_{j, a}$.

$\mathrm{Zu}$ jedem Gebiet $\Omega_{j}$ setzen wir die Existenz von Wellenzahlen $\kappa_{j} \operatorname{mit} \operatorname{Im}\left(\kappa_{j}\right)>0$ oder $\kappa_{j} \in \mathbb{R}_{+}$und zu jedem Rand $\Gamma_{j}$ die Existenz von Konstanten $\rho_{j} \in \mathbb{C} \backslash\{0,-1\}$ voraus. Die Konstanten $\rho_{j}$ gehen als Übergangskonstanten in die Neumann-Randwerte beim Transmissionsproblem ein.

Um die Notation etwas abzukürzen, führen wir Cauchy-Randoperatoren ein: Sei $u_{a / i}$ eine im Äußeren oder Inneren des Randes $\Gamma_{j}$ definierte und stetig bis in den Rand differenzierbare Funktion, dann betrachten wir folgende Randoperatoren:

$$
\mathcal{R}_{C, \Gamma_{j}, a} u_{a}:=\left(\begin{array}{c}
\left.u_{a}\right|_{\Gamma_{j}} \\
\left.\frac{\partial u_{a}}{\partial \nu}\right|_{\Gamma_{j}}
\end{array}\right)=\left(\begin{array}{c}
\mathcal{R}_{D, \Gamma_{j}, a} u_{a} \\
\mathcal{R}_{N, \Gamma_{j}, a} u_{a}
\end{array}\right), \quad \mathcal{R}_{C, \Gamma_{j}, i} u_{i}:=\left(\begin{array}{c}
\left.u_{i}\right|_{\Gamma_{j}} \\
\left.\frac{\partial u_{i}}{\partial \nu}\right|_{\Gamma_{j}}
\end{array}\right)=\left(\begin{array}{l}
\mathcal{R}_{D, \Gamma_{j}, i} u_{i} \\
\mathcal{R}_{N, \Gamma_{j}, i} u_{i}
\end{array}\right) .
$$

Das Bild der Operatoren sind also gerade die Cauchy-Werte einer Funktion. Mit $\mathcal{R}_{D, \Gamma_{j}, a}$ bezeichnen wir den Dirichlet-Randwertoperator bzgl. Funktionen im Außenraum von 
$\Gamma_{j}$. Entsprechend sind die Operatoren $\mathcal{R}_{N, \Gamma_{j}, a}, \mathcal{R}_{D, \Gamma_{j}, i}$ und $\mathcal{R}_{N, \Gamma_{j}, i}$ zu verstehen. Mit diesen beiden Cauchy-Randwertoperatoren kann folgender Transmissionsrandwertoperator bzgl. des Randes $\Gamma_{j}$ definiert werden (hierbei sei $u_{v(j)}$ die Funktion in $\Omega_{v(j)}$ und $u_{j}$ die Funktion in $\Omega_{j}$ ):

$$
\begin{aligned}
\mathcal{R}_{T, \Gamma_{j}, \rho_{j}}\left(u_{j}, u_{v(j)}\right) & :=\left(\begin{array}{c}
\left.\left(u_{j}-u_{v(j)}\right)\right|_{\Gamma_{j}} \\
\left.\left(\frac{\partial u_{j}}{\partial \nu}-\rho_{j} \frac{\partial u_{v(j)}}{\partial \nu}\right)\right|_{\Gamma_{j}}
\end{array}\right)=\mathcal{R}_{C, \Gamma_{j}, i} u_{j}-I_{\rho_{j}} \mathcal{R}_{C, \Gamma_{j}, a} u_{v(j)} \text { mit } \\
I_{\rho_{j}} & :=\left(\begin{array}{cc}
1 & 0 \\
0 & \rho_{j}
\end{array}\right) .
\end{aligned}
$$

Wichtig wird im folgenden die Linearität des Operators $\mathcal{R}_{T, \Gamma_{j}, \rho_{j}}$ sein: für $u, v \in$ $C^{1}\left(\overline{\Omega_{j}}\right) \times C^{1}\left(\overline{\Omega_{v(j)}}\right)$ und $\gamma, \delta \in \mathbb{C}$ gilt nämlich

$$
\mathcal{R}_{T, \Gamma_{j}, \rho_{j}}(\gamma u+\delta v)=\gamma \mathcal{R}_{T, \Gamma_{j}, \rho_{j}} u+\delta \mathcal{R}_{T, \Gamma_{j}, \rho_{j}} v .
$$

Für Operatoren $P: X \rightarrow C^{1}\left(\overline{\Omega_{j}}\right)$ und $Q: X \rightarrow C^{1}\left(\overline{\Omega_{v(j)}}\right)$ schreiben wir auch $\mathcal{R}_{T, \Gamma_{j}, \rho_{j}}(P, Q) \varphi:=\mathcal{R}_{T, \Gamma_{j}, \rho_{j}}(P \varphi, Q \varphi)$. Weiterhin betrachten wir folgende Räume:

$$
\begin{aligned}
C^{m, j}(\Gamma) & :=C^{m}\left(\Gamma_{1}\right) \times C^{j}\left(\Gamma_{1}\right) \times \ldots \times C^{m}\left(\Gamma_{L}\right) \times C^{j}\left(\Gamma_{L}\right), \quad j \in \mathbb{N}_{0}, \\
C^{m, j, \alpha}(\Gamma) & :=C^{m, \alpha}\left(\Gamma_{1}\right) \times C^{j, \alpha}\left(\Gamma_{1}\right) \times \ldots \times C^{m, \alpha}\left(\Gamma_{L}\right) \times C^{j, \alpha}\left(\Gamma_{L}\right), \quad j \in \mathbb{N}_{0}, \\
\hat{C}^{m, \alpha} & :=\hat{C}^{m, \alpha}\left(\overline{\Omega_{0}}\right) \times C^{m, \alpha}\left(\overline{\Omega_{1}}\right) \times \ldots \times C^{m, \alpha}\left(\overline{\Omega_{L}}\right), \\
C_{0}^{m, \alpha} & :=C_{0}^{m, \alpha}\left(\overline{\Omega_{0}}\right) \times C^{m, \alpha}\left(\overline{\Omega_{1}}\right) \times \ldots \times C^{m, \alpha}\left(\overline{\Omega_{L}}\right) .
\end{aligned}
$$

Hierbei wurden $\hat{C}^{m, \alpha}\left(\overline{\Omega_{0}}\right)$ und $C_{0}^{m, \alpha}\left(\overline{\Omega_{0}}\right)$ im letzten Kapitel definiert. Zudem benötigen wir die Operatoren

$$
\begin{gathered}
\mathcal{L}_{T}:\left\{\begin{array}{cl}
C^{2}\left(\overline{\Omega_{0}}\right) \times \ldots \times C^{2}\left(\overline{\Omega_{L}}\right) & \longrightarrow C\left(\overline{\Omega_{0}}\right) \times \ldots \times C\left(\overline{\Omega_{L}}\right) \\
u & \longmapsto\left(\Delta u_{j}+\kappa_{j}^{2} u_{j}\right)_{j=0}^{L}
\end{array}\right. \\
\mathcal{R}_{T}:\left\{\begin{array}{cl}
C^{1}\left(\overline{\Omega_{0}}\right) \times \ldots \times C^{1}\left(\overline{\Omega_{L}}\right) & \longrightarrow C^{1,0}(\Gamma) \\
u & \longmapsto\left(\mathcal{R}_{T, \Gamma_{j}, \rho_{j}}\left(u_{j}, u_{v(j)}\right)\right)_{j=1}^{L}
\end{array}\right.
\end{gathered}
$$

Es wird nun folgendes Problem betrachtet:

Problem 5.1. Sei $f \in C^{m, m-1, \alpha}(\Gamma)$. Gesucht ist $u \in \hat{C}^{m, \alpha}$ mit

$$
\mathcal{L}_{T} u=0 \text { und } \mathcal{R}_{T} u=I_{\rho} f
$$

(Hierbei sei $\left.\left(I_{\rho} f\right)_{j}:=I_{\rho_{j}} f_{j}\right)$.

\subsection{Eindeutigkeit}

Zunächst weisen wir nach, daß das Problem unter geeigneten Voraussetzungen an die auftretenden Konstanten höchstens eine Lösung besitzt. Hierfür werden zwei hinreichende Voraussetzungen angegeben. Die Beweise wurden in ähnlicher Form für den 
Fall eines einzigen, zusammenhängenden Randes auch bereits in [40], Theorem 3.1, und [34], Seite 309, geführt. Um Eindeutigkeitsaussagen zu beweisen, benötigen wir geeignete Voraussetzungen an die auftretenden Konstanten. Hierzu betrachten wir die Menge $\mathfrak{A}$ mit

$$
\mathfrak{A}:=\left\{\left(j_{l}\right)_{l=1}^{q} \in\{1, \ldots, L\}^{q}: 1 \leq q \leq L, v\left(j_{1}\right)=0, v\left(j_{l}\right)=j_{l-1}, l=2, \ldots, q\right\} .
$$

Für $\left(j_{l}\right)_{l=1}^{q} \in \mathfrak{A}$ beschreibt die Folge $\left(\Gamma_{j_{l}}\right)_{l=1}^{q}$ eine Folge von direkt ineinanderliegenden Rändern mit $\Gamma_{j_{1}} \subset \partial \Omega_{0}$.

Satz 5.2. Für alle $\left(j_{l}\right)_{l=1}^{q} \in \mathfrak{A}$ gelte

$$
\operatorname{Im}\left(\frac{\overline{\kappa_{0}} \kappa_{j_{l}}^{2}}{\rho_{j_{1}} \cdot \ldots \cdot \rho_{j_{l}}}\right) \geq 0, \quad \operatorname{Im}\left(\frac{\kappa_{0}}{\overline{\rho_{j_{1}} \cdot \ldots \cdot \rho_{j_{l}}}}\right) \geq 0 .
$$

Dann hat das Problem 5.1 höchstens eine Lösung.

Beweis. Sei $u:=\left(u_{j}\right)_{j=0}^{L}$ eine Lösung des homogenen Problems, dann ergibt sich unter Verwendung des ersten Greenschen Satzes und der Randbedingungen $\mathcal{R}_{T} u=0$ die folgende Ungleichung:

$$
\begin{aligned}
& \operatorname{Im}\left(\kappa_{0} \int_{\partial \Omega_{0}} u_{0} \frac{\partial \overline{u_{0}}}{\partial \nu} \mathrm{ds}\right)=\sum_{\substack{j \in\{1, \ldots, L\} \\
v(j)=0}} \operatorname{Im}\left(\frac{\kappa_{0}}{\overline{\rho_{j}}} \int_{\Gamma_{j}} u_{j} \frac{\partial \overline{u_{j}}}{\partial \nu} \mathrm{ds}\right) \\
& =\sum_{\substack{j \in\{1, \ldots, L\} \\
v(j)=0, n(j)=\emptyset}}\left(\operatorname{Im}\left(\frac{\overline{\kappa_{0}} \kappa_{j}^{2}}{\rho_{j}}\right)\left\|u_{j}\right\|_{L^{2}\left(\Omega_{j}\right)}^{2}+\operatorname{Im}\left(\frac{\kappa_{0}}{\overline{\rho_{j}}}\right)\left\|\nabla u_{j}\right\|_{L^{2}\left(\Omega_{j}\right)}^{2}\right) \\
& +\sum_{\substack{j \in\{1, \ldots, L\} \\
v(j)=0, n(j) \neq \emptyset}}\left(\operatorname{Im}\left(\frac{\kappa_{0}}{\overline{\rho_{j}}} \int_{\partial \Omega_{j}} u_{j} \frac{\partial \overline{u_{j}}}{\partial \nu} \mathrm{ds}\right)+\sum_{l \in n(j)} \operatorname{Im}\left(\frac{\kappa_{0}}{\overline{\rho_{j} \rho_{l}}} \int_{\Gamma_{l}} u_{l} \frac{\partial \overline{u_{l}}}{\partial \nu} \mathrm{ds}\right)\right) \\
& =\sum_{\substack{j \in\{1, \ldots, L\} \\
v(j)=0}}\left(\operatorname{Im}\left(\frac{\overline{\kappa_{0}} \kappa_{j}^{2}}{\rho_{j}}\right)\left\|u_{j}\right\|_{L^{2}\left(\Omega_{j}\right)}^{2}+\operatorname{Im}\left(\frac{\kappa_{0}}{\overline{\rho_{j}}}\right)\left\|\nabla u_{j}\right\|_{L^{2}\left(\Omega_{j}\right)}^{2}\right) \\
& +\sum_{\substack{j \in\{1, \ldots, L\} \\
v(j)=0, n(j) \neq \emptyset}} \sum_{l \in n(j)} \operatorname{Im}\left(\frac{\kappa_{0}}{\overline{\rho_{j} \rho_{l}}} \int_{\Gamma_{l}} u_{l} \frac{\partial \overline{u_{l}}}{\partial \nu} \mathrm{ds}\right) \\
& =\ldots= \\
& =\sum_{\left(j_{l}\right)_{l=1}^{q} \in \mathfrak{A}}\left(\operatorname{Im}\left(\frac{\overline{\kappa_{0}} \kappa_{j_{l}}^{2}}{\rho_{j_{1}} \cdot \ldots \cdot \rho_{j_{l}}}\right)\left\|u_{j_{l}}\right\|_{L^{2}\left(\Omega_{j_{l}}\right)}^{2}+\operatorname{Im}\left(\frac{\kappa_{0}}{\overline{\rho_{j_{1}} \cdot \cdots \cdot \rho_{j_{l}}}}\right)\left\|\nabla u_{j_{l}}\right\|_{L^{2}\left(\Omega_{j_{l}}\right)}^{2}\right) \\
& \geq 0 \text {. }
\end{aligned}
$$

Somit ist Theorem 3.12 aus [6] anwendbar (die Aussage des Korollars bleibt auch für nichtzusammenhängenden Rand $\partial \Omega_{0}$ gültig) und es folgt $u_{0}=0$ in $\Omega_{0}$. Für $u_{j}$ mit 
$v(j)=0$ folgt aus den homogenen Transmissionsrandbedingungen, daß die CauchyWerte von $u_{j}$ auf $\Gamma_{j}$ verschwinden. Nun verwendet man den Greenschen Darstellungssatz. Falls $n(j)=\emptyset\left(\right.$ d.h. $\left.\partial \Omega_{j}=\Gamma_{j}\right)$, folgt sofort $u_{j}=0$. Anderenfalls gilt

$$
u_{j}(x)=\int_{\partial \Omega_{j} \backslash \Gamma_{j}} \frac{\partial \Phi(x, y)}{\partial \nu(y)} u_{j}(y)-\frac{\partial u_{j}}{\partial \nu}(y) \Phi(x, y) \operatorname{ds}(y), \quad x \in \Omega_{j} .
$$

Mit Hilfe dieser Darstellung kann $u_{j}$ zu einer Funktion in $\overline{\Omega_{j, a}} \cap \Omega_{j}$ fortgesetzt werden. Aus dem zweiten Greenschen Satz folgt $\left.u_{j}\right|_{\Omega_{j, a}}=0$. Die Analytizität von $u_{j}$ in $\overline{\Omega_{j, a}} \cup \Omega_{j}$ bedingt dann $\left.u\right|_{\Omega_{j}}=0$. Der Greensche Darstellungssatz kann in dieser Situation also als einfache Alternative zum Holmgrenschen Satz verwendet werden (vgl. Theorem 2.1.2.2 in $[38])$.

Für die verbleibenden Funktionen kann in gleicher Weise gefolgert werden, so daß die Aussage bewiesen ist.

In [34], Seite 310, werden einige Beispiele angegeben, für die dieser Eindeutigkeitssatz gültig ist. Erwähnt wird z.B. das Erhitzen von Fleisch in der Mikrowelle. Reduziert man ein Randwertproblem zu den zeitharmonischen Maxwell-Gleichungen im $\mathbb{R}^{3}$ durch geeignete Annahmen auf ein zweidimensionales, skalares Transmissionsproblem bei der Helmholtz-Gleichung, so ergeben sich die Bedingungen $\operatorname{Im}\left(\kappa_{j}\right) \geq 0, \operatorname{Re}\left(\kappa_{j}\right)>0$ und $\rho_{j}>0$ (vgl. Kapitel 1). Für diese Situation wollen wir einen weiteren Eindeutigkeitsbeweis angeben.

Satz 5.3. Sei $\operatorname{Im}\left(\kappa_{0}\right) \operatorname{Re}\left(\kappa_{0}\right) \geq 0, \operatorname{Re}\left(\kappa_{0}\right) \geq 0$ und für jede Folge $\left(j_{l}\right)_{l=1}^{q} \in \mathfrak{A}$ gelte

$$
\operatorname{Im}\left(\frac{\kappa_{j_{l}}^{2}}{\rho_{j_{1}} \cdot \ldots \cdot \rho_{j_{l}}}\right) \geq 0, \quad \operatorname{Im}\left(\frac{1}{\overline{\rho_{j_{1}} \cdot \ldots \cdot \rho_{j_{l}}}}\right) \geq 0 .
$$

Weiterhin sei zumindest eine dieser Ungleichungen strikt. Dann hat das Problem 5.1 höchstens eine Lösung.

Beweis. Sei $\left(u_{j}\right)_{j=0}^{L}$ wiederum eine Lösung des homogenen Problems. Sei $\Omega_{R}:=B_{R} \cap \Omega_{0}$ (die Normale von $\Omega_{R}$ zeige in das Äußere von $\Omega_{R}$ ), dann ergibt sich aus dem ersten Greenschen Satz folgende Gleichung:

$$
\begin{aligned}
& 2 \operatorname{Im}\left(\kappa_{0}\right) \operatorname{Re}\left(\kappa_{0}\right)\left\|u_{0}\right\|_{L^{2}\left(\Omega_{R}\right)}^{2}=\operatorname{Im}\left(\left\|\nabla u_{0}\right\|_{L^{2}\left(\Omega_{R}\right)}^{2}-{\overline{\kappa_{0}}}^{2}\left\|u_{0}\right\|_{L^{2}\left(\Omega_{R}\right)}^{2}\right)=\operatorname{Im}\left(\int_{\partial \Omega_{R}} u_{0} \frac{\partial \overline{u_{0}}}{\partial \nu} \mathrm{ds}\right) \\
& =\operatorname{Im}(\int_{\partial B_{R}} \underbrace{u_{0} \overline{\left(\frac{\partial u_{0}}{\partial \nu}-i \kappa_{0} u_{0}\right)}}_{=o\left(1 / R^{d-1}\right)}+\overline{i \kappa_{0}}\left|u_{0}\right|^{2} \mathrm{ds})-\operatorname{Im}\left(\int_{\partial \Omega_{0}} u_{0} \frac{\partial \overline{u_{0}}}{\partial \nu} \mathrm{ds}\right) \\
& =-\operatorname{Re}\left(\kappa_{0}\right)\left\|u_{0}\right\|_{L^{2}\left(\partial B_{R}\right)}^{2}-\operatorname{Im}\left(\int_{\partial \Omega_{0}} u_{0} \frac{\partial \overline{u_{0}}}{\partial \nu} \mathrm{ds}\right)+\mathrm{o}(1), \quad R \rightarrow \infty .
\end{aligned}
$$


Die Asymptotik ergibt sich aus $u_{0}(x)=\mathrm{O}\left(1 /|x|^{(d-1) / 2}\right)$ für $x \rightarrow \infty$ und der Sommerfeldschen Ausstrahlungsbedingung. Insgesamt folgt somit, wenn man $\operatorname{Im}\left(\int_{\partial \Omega_{0}} u_{0} \frac{\partial \overline{u_{0}}}{\partial \nu} \mathrm{ds}\right)$ wie im letzten Satz darstellt:

$$
\begin{aligned}
& 2 \operatorname{Im}\left(\kappa_{0}\right) \operatorname{Re}\left(\kappa_{0}\right)\left\|u_{0}\right\|_{L^{2}\left(\Omega_{R}\right)}^{2}+\operatorname{Re}\left(\kappa_{0}\right)\left\|u_{0}\right\|_{L^{2}\left(\partial B_{R}\right)}^{2}+ \\
& \sum_{\left(j_{l}\right)_{l=1}^{q} \in \mathfrak{A}}\left(\operatorname{Im}\left(\frac{\kappa_{j_{l}}^{2}}{\rho_{j_{1}} \cdot \cdots \cdot \rho_{j_{l}}}\right)\left\|u_{j_{l}}\right\|_{L^{2}\left(\Omega_{j_{l}}\right)}^{2}+\operatorname{Im}\left(\frac{1}{\overline{\rho_{j_{1}} \cdot \cdots \cdot \rho_{j_{l}}}}\right)\left\|\nabla u_{j_{l}}\right\|_{L^{2}\left(\Omega_{j_{l}}\right)}^{2}\right) \\
& =\mathrm{o}(1) \text { für } R \rightarrow \infty .
\end{aligned}
$$

Alle Terme der linken Seite sind nicht negativ und müssen daher für $R \rightarrow \infty$ verschwinden. Nach Voraussetzung ist zumindest eine Ungleichung strikt. Falls $\operatorname{Re}\left(\kappa_{0}\right)>0$, so folgt aus dem Rellich-Lemma $\left.u_{0}\right|_{\Omega_{0}}=0$ und damit die Behauptung wie im vorherigen Satz. Folgt aus einer Ungleichung $\left.u_{j}\right|_{\Omega_{j}}=0$ für ein $j=1, \ldots, L$, so zeigt man mit Hilfe des Greenschen Darstellungssatzes wie im letzten Satz die Gültigkeit der Behauptung. Gilt schließlich $\left\|\nabla u_{j_{l}}\right\|_{L^{2}\left(\Omega_{j_{l}}\right)}=0$, so ist $u_{j_{l}}$ auf $\Omega_{j_{l}}$ konstant und verschwindet somit als Lösung der Helmholtzgleichung identisch, d.h. auch in diesem Fall kann wie davor gefolgert werden.

\subsection{Potentialansatz}

In diesem Teil des Kapitels wird gezeigt, daß das Problem 5.1 eine Lösung hat. Dies geschieht konstruktiv mit Hilfe eines Potentialansatzes. Im Anschluß daran werden wir auch noch zwei andere Ansätze untersuchen. Der Potentialansatz wurde für den Fall eines Randes bereits in dem Artikel [40] (vgl. auch [6], Paragraph 3.8) verwendet. Wir werden stets $\Gamma \in C^{m, \alpha}$ und $f \in C^{m, m-1, \alpha}(\Gamma)\left(m \in \mathbb{N}_{\geq 2}\right)$ voraussetzen. Für jeden auftretenden Rand $\Gamma_{j}, j=1, \ldots, L$ werden nun geeignete Potentialoperatoren $\mathcal{P}_{j, a}$ (mit $\kappa_{v(j)}$ als Wellenzahl der Grundlösung) und $\mathcal{P}_{j, i}$ (mit $\kappa_{j}$ als Wellenzahl der Grundlösung) definiert (wobei $\Gamma_{j}$ in beiden Fällen der Integrationsrand der Integraloperatoren ist):

$$
\begin{aligned}
\mathcal{P}_{j, a} \varphi_{j} & :=\mathcal{K}_{j, a} \varphi_{j, 1}+d_{j} \mathcal{S}_{j, a} \varphi_{j, 2}, \quad d_{j} \in \mathbb{C} \backslash\left\{-1 / \rho_{j}\right\}, \\
\mathcal{P}_{j, i} \varphi_{j}: & :=\rho_{j} \mathcal{K}_{j, i} \varphi_{j, 1}+\mathcal{S}_{j, i} \varphi_{j, 2} .
\end{aligned}
$$

Die Dichte $\varphi_{j}=\left(\varphi_{j, 1}, \varphi_{j, 2}\right)$ wählen wir aus $C^{m, \alpha}\left(\Gamma_{j}\right) \times C^{m-1, \alpha}\left(\Gamma_{j}\right)$. Somit ergeben sich folgende Cauchyrandwerte für die Operatoren:

$$
\begin{aligned}
\mathcal{R}_{C, \Gamma_{j}, a / i} \mathcal{P}_{j, a} \varphi_{j} & =\left[\left(\begin{array}{cc}
K_{j, a} & d_{j} S_{j, a} \\
T_{j, a} & d_{j} K_{j, a}^{*}
\end{array}\right)+\left(\begin{array}{cc} 
\pm \frac{1}{2} & 0 \\
0 & \mp \frac{d_{j}}{2}
\end{array}\right)\right] \varphi_{j}, \\
\mathcal{R}_{C, \Gamma_{j}, a / i} \mathcal{P}_{j, i} \varphi_{j} & =\left[\left(\begin{array}{ll}
\rho_{j} K_{j, i} & S_{j, i} \\
\rho_{j} T_{j, i} & K_{j, i}^{*}
\end{array}\right)+\left(\begin{array}{cc} 
\pm \frac{\rho_{j}}{2} & 0 \\
0 & \mp \frac{1}{2}
\end{array}\right)\right] \varphi_{j}
\end{aligned}
$$

Hierbei bezeichnen wir mit $\mathcal{R}_{C, \Gamma_{j}, a / i}$ entweder $\mathcal{R}_{C, \Gamma_{j}, a}$ oder $\mathcal{R}_{C, \Gamma_{j}, i}$. Nun wird folgender Ansatz gewählt:

$$
u_{j}:=\sum_{l \in n(j)} \mathcal{P}_{l, a} \varphi_{l}+\mathcal{P}_{j, i} \varphi_{j}, \quad j=0, \ldots, L .
$$


Hierbei sei $\mathcal{P}_{0, i} \varphi_{0}:=0$, d.h. für $u_{0}$ tritt der letzte Summand nicht auf und $\varphi:=$ $\left(\varphi_{j}\right)_{j=1}^{L} \in C^{m, m-1, \alpha}(\Gamma)$. Somit ist klar, daß die Funktionen die jeweiligen Helmholtzgleichungen lösen und die gewünschte Regularität haben. Weiterhin genügt $u_{0}$ der Sommerfeldschen Ausstrahlungsbedingung. Damit die Randbedingungen erfüllt werden, müssen für $j=1, \ldots, L$ folgende Gleichungen erfüllt sein:

$$
\begin{aligned}
\mathcal{R}_{T, \Gamma_{j}, \rho_{j}}\left(u_{j}, u_{v(j)}\right)= & \mathcal{R}_{T, \Gamma_{j}, \rho_{j}}\left(\sum_{l \in n(j)} \mathcal{P}_{l, a} \varphi_{l}+\mathcal{P}_{j, i} \varphi_{j}, \sum_{l \in n(v(j))} \mathcal{P}_{l, a} \varphi_{l}+\mathcal{P}_{v(j), i} \varphi_{v(j)}\right) \\
= & \sum_{l \in n(j)} \mathcal{R}_{T, \Gamma_{j}, \rho_{j}}\left(\mathcal{P}_{l, a}, 0\right) \varphi_{l}+\mathcal{R}_{T, \Gamma_{j}, \rho_{j}}\left(\mathcal{P}_{j, i}, \mathcal{P}_{j, a}\right) \varphi_{j} \\
& +\sum_{\substack{l \in n(v(j)) \\
l \neq j}} \mathcal{R}_{T, \Gamma_{j}, \rho_{j}}\left(0, \mathcal{P}_{l, a}\right) \varphi_{l}+\mathcal{R}_{T, \Gamma_{j}, \rho_{j}}\left(0, \mathcal{P}_{v(j), i}\right) \varphi_{v(j)} \\
\stackrel{!}{=} & I_{\rho_{j}} f_{j} .
\end{aligned}
$$

Faßt man diese Gleichungen zu einem System $M \varphi=I_{\rho} f$ zusammen, so ergeben sich die Diagonalelemente der Operatormatrix $M$ gerade aus den Termen $\mathcal{R}_{T, \Gamma_{j}, \rho_{j}}\left(\mathcal{P}_{j, i}, \mathcal{P}_{j, a}\right)$, $j=1, \ldots, L$. Daher haben sie folgende Form:

$$
\begin{aligned}
M_{j, j} & =\left(\begin{array}{cc}
\rho_{j} K_{j, i}-K_{j, a} & S_{j, i}-d_{j} S_{j, a} \\
\rho_{j}\left(T_{j, i}-T_{j, a}\right) & K_{j, i}^{*}-\rho_{j} d_{j} K_{j, a}^{*}
\end{array}\right)+\left(\begin{array}{cc}
-\frac{1}{2}\left(\rho_{j}+1\right) & 0 \\
0 & \frac{1}{2}\left(1+\rho_{j} d_{j}\right)
\end{array}\right) \\
& =\left(\begin{array}{cc}
\rho_{j} K_{j, i}-K_{j, a} & S_{j, i}-S_{j, a} \\
\rho_{j}\left(T_{j, i}-T_{j, a}\right) & K_{j, i}^{*}-\rho_{j} d_{j} K_{j, a}^{*}
\end{array}\right)+\left(\begin{array}{cc}
-\frac{1}{2}\left(\rho_{j}+1\right) & \left(1-d_{j}\right) S_{j, a} \\
0 & \frac{1}{2}\left(1+\rho_{j} d_{j}\right)
\end{array}\right) .
\end{aligned}
$$

Den ersten Summanden in (5.4) bezeichnen wir mit $M_{k, j, j}$, den zweiten mit $M_{d, j, j} . M$ hat also die Form $M=M_{d}+M_{k}$ mit einer oberen Dreiecksmatrix $M_{d}=\left(M_{d, j, l}\right)_{j, l=1}^{L}$, die in $C^{m, m-1, \alpha}(\Gamma)$ beschränkt invertierbar ist (hier werden die Voraussetzungen $\rho_{j} \neq-1$ und $d_{j} \neq-1 / \rho_{j}$ benötigt). Die Inverse eines Blocks $M_{d, j, j}$ ist hierbei gegeben durch

$$
M_{d, j, j}^{-1}=\left(\begin{array}{cc}
-\frac{2}{\rho_{j}+1} & \frac{4\left(1-d_{j}\right)}{\left(\rho_{j}+1\right)\left(1+\rho_{j} d_{j}\right)} S_{j, a} \\
0 & \frac{2}{1+\rho_{j} d_{j}}
\end{array}\right) .
$$

Weiterhin ist die Matrix $M_{k}=\left(M_{k, j, l}\right)_{j, l=1}^{L}$ in $C^{m, m-1, \alpha}(\Gamma)$ kompakt. Problematisch beim Nachweis der Kompaktheit sind nur die Diagonalelemente von $M_{k}$, denn die anderen Elemente bestehen aus Integraloperatoren mit glattem Kern (vgl. Satz 3.1). Die Kompaktheit der Operatormatrizen $M_{k, j, j}$ und die Beschränktheit von $M_{d, j, j}$ wird mit Satz 3.2 gezeigt. Somit ist die Riesz-Theorie anwendbar.

Satz 5.4. Sei das homogene Problem 5.1 nur trivial lösbar, dann existiert für das inhomogene Problem genau eine Lösung.

Beweis. Die Dichte $\varphi$ löse $M \varphi=0$. Dann löst $u=\left(u_{j}\right)_{j=0}^{L}$ definiert durch (5.1 das homogene Problem 5.1 und dies bedingt $\left.u_{j}\right|_{\overline{\Omega_{j}}}=0$ nach Voraussetzung. Aus den Sprung- 
beziehungen folgt für $j=1, \ldots, L$ :

$$
\begin{aligned}
\mathcal{R}_{C, \Gamma_{j}, a} u_{j} & =\mathcal{R}_{C, \Gamma_{j}, a} u_{j}-\mathcal{R}_{C, \Gamma_{j}, i} u_{j}=\left(\mathcal{R}_{C, \Gamma_{j}, a}-\mathcal{R}_{C, \Gamma_{j}, i}\right) \mathcal{P}_{j, i} \varphi_{j}=\rho_{j} I_{-1 / \rho_{j}} \varphi_{j} \\
-\mathcal{R}_{C, \Gamma_{j}, i} u_{v(j)} & =\mathcal{R}_{C, \Gamma_{j}, a} u_{v(j)}-\mathcal{R}_{C, \Gamma_{j}, i} u_{v(j)}=\left(\mathcal{R}_{C, \Gamma_{j}, a}-\mathcal{R}_{C, \Gamma_{j}, i}\right) \mathcal{P}_{j, a} \varphi_{j}=I_{-d_{j}} \varphi_{j}
\end{aligned}
$$

Hier wurde benutzt, daß nur bei Integraloperatoren mit Integrationsrand $\Gamma_{j}$ ein Sprung in den Cauchy-Daten bei $\Gamma_{j}$ auftritt. Der Beweistrick besteht nun darin, $u_{v(j)}$ als Potential in $\Omega_{j, i}$ und $u_{j}$ als Potential in $\Omega_{j, a}$ zu betrachten. Hier ergibt sich

$$
\begin{aligned}
\mathcal{R}_{T, \Gamma_{j}, d_{j} \rho_{j}}\left(-\rho_{j} u_{v(j)}, u_{j}\right) & =-\rho_{j} \mathcal{R}_{C, \Gamma_{j}, i} u_{v(j)}-I_{d_{j} \rho_{j}} \mathcal{R}_{C, \Gamma_{j}, a} u_{j} \\
& =\rho_{j} I_{-d_{j}} \varphi_{j}-\rho_{j} I_{-1 / \rho_{j}} I_{d_{j} \rho_{j}} \varphi_{j}=0, \quad j=1 \ldots, L .
\end{aligned}
$$

Die Konstanten $d_{j}$ in den Ansatzfunktionen sind noch frei wählbar. Wir legen sie nun durch $d_{j}:=\left(\overline{\kappa_{j}} \kappa_{v(j)}\right) / \rho_{j}$ fest. Damit sind die Voraussetzungen von Satz 5.2 für ein Transmissionsproblem in $\Omega_{j, a}\left(\right.$ mit Wellenzahl $\left.\kappa_{j}\right)$ und $\Omega_{j, i}\left(\right.$ mit Wellenzahl $\left.\kappa_{v(j)}\right)$ erfüllt - aus der Voraussetzung $\operatorname{Im}\left(\kappa_{j}\right) \geq 0$ bzw. $\kappa_{j} \in \mathbb{R}_{+}$folgt insbesondere $d_{j} \neq-1 / \rho_{j}-$ und daher folgt $\left.u_{j}\right|_{\Omega_{j, a}}=0$ und $\left.u_{v(j)}\right|_{\Omega_{j, i}}=0$ und somit aus den Sprungbeziehungen $\left.\varphi_{j}\right|_{\Gamma_{j}}=0$.

Bemerkung 5.5. Wir wollen kurz eine Möglichkeit angeben, die Operatormatrix mit den Gleichungen (5.3) zu analysieren. Hier nutzt man $S_{j, i}-d_{j} S_{j, a} \in$ $L\left(C^{m-1, \alpha}\left(\Gamma_{j}\right), C^{m, \alpha}\left(\Gamma_{j}\right)\right)$ und die Kompaktheit der Einbettung $C^{m, \alpha}\left(\Gamma_{j}\right) \hookrightarrow C^{m, \beta}\left(\Gamma_{j}\right)$ für $0<\beta<\alpha$ und kann somit folgern, daß die Abbildung

$$
M_{k, j, j}: C^{m, \beta}\left(\Gamma_{j}\right) \times C^{m-1, \alpha}\left(\Gamma_{j}\right) \rightarrow C^{m, \beta}\left(\Gamma_{j}\right) \times C^{m-1, \alpha}\left(\Gamma_{j}\right)
$$

kompakt ist. Somit ist auch hier die Riesz-Theorie anwendbar. Der Nachteil bei dieser Vorgehensweise liegt in der etwas geringeren Regularität $\left(C^{m, \beta}\left(\Gamma_{j}\right)\right.$ statt $\left.C^{m, \alpha}\left(\Gamma_{j}\right)\right)$. Zudem wird die schärfere Regularitätsaussage im nächsten Kapitel für Differenzierbarkeitsaussagen benötigt. Allerdings werden wir diese zweite Möglichkeit für eine Konvergenzanalyse eines Verfahrens zur numerischen Berechnung der Integraloperatoren nutzen.

Als nächstes soll ein Algorithmus für die Implementation der Operatormatrix $M$ angegeben werden. Aus der Gleichung 5.2 ergibt sich folgendes Verfahren zur Initialisierung der Einträge in Pseudocode:

for $(j=1, \ldots, L)$

- $\operatorname{for}(1=1, \ldots, L)$

$$
\begin{aligned}
& \text { - if }(j=1)\left\{M(j, j)=\mathcal{R}_{T, \Gamma_{j}, \rho_{j}}\left(\mathcal{P}_{j, i}, \mathcal{P}_{j, a}\right)\right\} \\
& \text { - else if }(j=\mathrm{v}(1)) \quad\left\{M(j, l)=\mathcal{R}_{T, \Gamma_{j}, \rho_{j}}\left(\mathcal{P}_{l, a}, 0\right)\right\} \\
& \text { - else if }(\mathrm{v}(\mathrm{j})=\mathrm{v}(1)) \quad\left\{M(j, l)=\mathcal{R}_{T, \Gamma_{j}, \rho_{j}}\left(0, \mathcal{P}_{l, a}\right)\right\} \\
& \text { - else if }(\mathrm{v}(\mathrm{j})=\mathrm{l}) \quad\left\{M(j, l)=\mathcal{R}_{T, \Gamma_{j}, \rho_{j}}\left(0, \mathcal{P}_{l, i}\right)\right\}
\end{aligned}
$$




$$
\text { - else }\{M(j, l)=0\}
$$

Dies kann noch etwas kompakter geschrieben werden, indem man die Voraussetzung $v(j)<j$ ausnutzt:

for $(j=1, \ldots, L)$

- $\operatorname{for}(1=1, \ldots, j-1)$

- if $(\mathrm{v}(\mathrm{j})=\mathrm{v}(\mathrm{l})) \quad\left\{M(j, l)=\mathcal{R}_{T, \Gamma_{j}, \rho_{j}}\left(0, \mathcal{P}_{l, a}\right) ; M(l, j)=\mathcal{R}_{T, \Gamma_{l}, \rho_{l}}\left(0, \mathcal{P}_{j, a}\right)\right\}$

- if (v(j)=1) $\left\{M(j, l)=\mathcal{R}_{T, \Gamma_{j}, \rho_{j}}\left(0, \mathcal{P}_{l, i}\right) ; M(l, j)=\mathcal{R}_{T, \Gamma_{l}, \rho_{l}}\left(\mathcal{P}_{j, a}, 0\right)\right\}$

- else $\{M(j, l)=0 ; \quad M(l, j)=0\}$

- $M(j, j)=\mathcal{R}_{T, \Gamma_{j}, \rho_{j}}\left(\mathcal{P}_{j, i}, \mathcal{P}_{j, a}\right)$

\subsection{Greenscher Ansatz}

Als nächstes untersuchen wir den Greenschen Ansatz zur Lösung des Problems. Grundlage ist hierbei der Greensche Darstellungssatz. Für den Rand $\Gamma_{j}$ definiert man die Potentialoperatoren $\mathcal{G}_{j, a}, \hat{\mathcal{G}}_{j, i}$ und $\mathcal{G}_{j, i}$ durch

$$
\begin{aligned}
\mathcal{G}_{j, a} \varphi_{j} & :=\mathcal{K}_{j, a} \varphi_{j, 1}-\mathcal{S}_{j, a} \varphi_{j, 2}, \\
\hat{\mathcal{G}}_{j, i} \varphi_{j} & :=-\mathcal{K}_{j, i} \varphi_{j, 1}+\mathcal{S}_{j, i} \varphi_{j, 2}, \\
\mathcal{G}_{j, i} & :=\hat{\mathcal{G}}_{j, i} I_{\rho_{j}} .
\end{aligned}
$$

Für $\mathcal{G}_{j, a}$ und $\mathcal{G}_{j, i}$ ergeben sich folgende Cauchy-Randwerte:

$$
\begin{aligned}
\mathcal{R}_{C, \Gamma_{j}, a / i} \mathcal{G}_{j, a} \varphi_{j} & =\left[\left(\begin{array}{cc}
K_{j, a} & -S_{j, a} \\
T_{j, a} & -K_{j, a}^{*}
\end{array}\right)+\left(\begin{array}{cc} 
\pm \frac{1}{2} & 0 \\
0 & \pm \frac{1}{2}
\end{array}\right)\right] \varphi_{j}, \\
\mathcal{R}_{C, \Gamma_{j}, a / i} \mathcal{G}_{j, i} \varphi_{j} & =\left[\left(\begin{array}{cc}
-K_{j, i} & \rho_{j} S_{j, i} \\
-T_{j, i} & \rho_{j} K_{j, i}^{*}
\end{array}\right)+\left(\begin{array}{cc}
\mp \frac{1}{2} & 0 \\
0 & \mp \frac{\rho_{j}}{2}
\end{array}\right)\right] \varphi_{j}
\end{aligned}
$$

Zur Motivation des Ansatzes nehmen wir an, daß $u$ Lösung von Problem 5.1 ist. Aus den Greenschen Darstellungssätzen folgt dann:

$$
u_{j}=\sum_{l \in n(j)} \mathcal{G}_{l, a} \mathcal{R}_{C, \Gamma_{l}, a} u_{j}+\hat{\mathcal{G}}_{j, i} \mathcal{R}_{C, \Gamma_{j}, i} u_{j}, \quad j=0, \ldots, L
$$

Hierbei sei $\hat{\mathcal{G}}_{0, i} \mathcal{R}_{C, \Gamma_{0}, i} u_{0}:=0$. Die Randbedingung $\mathcal{R}_{T} u=I_{\rho} f$ (d.h. $\mathcal{R}_{C, \Gamma_{j}, i} u_{j}=$ $\left.I_{\rho_{j}}\left(\mathcal{R}_{C, \Gamma_{j}, a} u_{v(j)}+f_{j}\right), j=1, \ldots, L\right)$ führt weiterhin auf die Gleichungen

$$
u_{j}=\sum_{l \in n(j)} \mathcal{G}_{l, a} \mathcal{R}_{C, \Gamma_{l}, a} u_{j}+\mathcal{G}_{j, i}\left(\mathcal{R}_{C, \Gamma_{j}, a} u_{v(j)}+f_{j}\right), \quad j=0, \ldots, L .
$$


Die Sprungbeziehungen ergeben für $j=1, \ldots, L$ folgende Gleichungen:

$$
\begin{aligned}
\mathcal{R}_{C, \Gamma_{j}, a} u_{j}-\mathcal{R}_{C, \Gamma_{j}, i} u_{j} & =\left(\mathcal{R}_{C, \Gamma_{j}, a}-\mathcal{R}_{C, \Gamma_{j}, i}\right) \mathcal{G}_{j, i}\left(\mathcal{R}_{C, \Gamma_{j}, a} u_{v(j)}+f_{j}\right) \\
& =-I_{\rho_{j}}\left(\mathcal{R}_{C, \Gamma_{j}, a} u_{v(j)}+f_{j}\right) \\
\mathcal{R}_{C, \Gamma_{j}, a} u_{v(j)}-\mathcal{R}_{C, \Gamma_{j}, i} u_{v(j)} & =\left(\mathcal{R}_{C, \Gamma_{j}, a}-\mathcal{R}_{C, \Gamma_{j}, i}\right) \mathcal{G}_{j, a} \mathcal{R}_{C, \Gamma_{j}, a} u_{v(j)} \\
& =\mathcal{R}_{C, \Gamma_{j}, a} u_{v(j)} .
\end{aligned}
$$

Notwendigerweise gilt daher

$$
\mathcal{R}_{C, \Gamma_{j}, i} u_{v(j)}=0, \quad \mathcal{R}_{C, \Gamma_{j}, a} u_{j}=0, \quad j=1, \ldots, L .
$$

Nun gibt es verschiedene Möglichkeiten aus den Gleichungen (5.5) ein System für die Dichten aufzustellen (vgl. [34], Abschnitt 4.2, für den Fall einer Randkurve). Im folgenden wählen wir eine Variante die zu einer Operatormatrix $M$ führt, deren Einträge Operatoren mit schwach singulären Kernen sind:

$$
\mathcal{R}_{C, \Gamma_{j}, i} u_{v(j)}+\mathcal{R}_{C, \Gamma_{j}, a} u_{j}=\mathcal{R}_{T, \Gamma_{j}, 1}\left(u_{v(j)},-u_{j}\right)=0, \quad j=1, \ldots, L .
$$

Sei nun $\varphi_{j}:=\mathcal{R}_{C, \Gamma_{j}, a} u_{v(j)},=1, \ldots, L$, dann ergibt sich folgendes Gleichungssystem:

$$
\begin{aligned}
\sum_{\substack{l \in n(v(j)) \\
l \neq j}} \mathcal{R}_{T, \Gamma_{j}, 1}\left(\mathcal{G}_{l, a}, 0\right) \varphi_{l}+\mathcal{R}_{T, \Gamma_{j}, 1}\left(\mathcal{G}_{j, a},\right. & \left.-\mathcal{G}_{j, i}\right) \varphi_{j} \\
+ & \mathcal{R}_{T, \Gamma_{j}, 1}\left(\mathcal{G}_{v(j), i}, 0\right) \varphi_{v(j)}-\sum_{l \in n(j)} \mathcal{R}_{T, \Gamma_{j}, 1}\left(0, \mathcal{G}_{l, a}\right) \varphi_{l} \\
& =-\mathcal{R}_{T, \Gamma_{j}, 1}\left(\mathcal{G}_{v(j), i}, 0\right) f_{v(j)}+\mathcal{R}_{T, \Gamma_{j}, 1}\left(0, \mathcal{G}_{j, i}\right) f_{j} .
\end{aligned}
$$

Dieses System bezeichnen wir mit $M \varphi=Q f$. Für die Diagonalelemente ergibt sich folgende Darstellung:

$$
\begin{aligned}
M_{j, j} & =\left(\begin{array}{cc}
K_{j, a}-K_{j, i} & \rho_{j} S_{j, i}-S_{j, a} \\
T_{j, a}-T_{j, i} & \rho_{j} K_{j, i}^{*}-K_{j, a}^{*}
\end{array}\right)+\left(\begin{array}{cc}
-1 & 0 \\
0 & -\frac{1}{2}\left(\rho_{j}+1\right)
\end{array}\right), \\
& =\left(\begin{array}{cc}
K_{j, a}-K_{j, i} & \rho_{j}\left(S_{j, i}-S_{j, a}\right) \\
T_{j, a}-T_{j, i} & \rho_{j} K_{j, i}^{*}-K_{j, a}^{*}
\end{array}\right)+\left(\begin{array}{cc}
-1 & \left(\rho_{j}-1\right) S_{j, a} \\
0 & -\frac{1}{2}\left(\rho_{j}+1\right)
\end{array}\right), \\
& \\
Q_{j, j}= & \left(\begin{array}{ll}
K_{j, i} & -\rho_{j} S_{j, i} \\
T_{j, i} & -\rho_{j} K_{j, i}^{*}
\end{array}\right)+\left(\begin{array}{ll}
\frac{1}{2} & 0 \\
0 & \frac{\rho_{j}}{2}
\end{array}\right) .
\end{aligned}
$$

Wie beim Potentialansatz gilt $M=M_{d}+M_{k}$ mit einer oberen Dreiecksmatrix $M_{d}$, die in $C^{m, m-1, \alpha}(\Gamma)$ beschränkt ist, und einem in $C^{m, m-1, \alpha}(\Gamma)$ kompakten Anteil $M_{k}$. Dies kann wiederum mit Satz 3.2 gezeigt werden. Somit ist auch hier die Riesz-Theorie anwendbar.

Satz 5.6. Sei das homogene Transmissionsproblem mit Wellenzahl $\kappa_{j}$ in $\Omega_{j, a}, \kappa_{v(j)}$ in $\Omega_{j, i}$ und $\rho=1$ nur trivial lösbar (für $j=1, \ldots, L$ ). Falls $\varphi$ Lösung von $M \varphi=Q f$ ist, dann löst u definiert durch

$$
u_{j}=\sum_{l \in n(j)} \mathcal{G}_{l, a} \varphi_{l}+\mathcal{G}_{j, i}\left(\varphi_{j}+f_{j}\right), \quad j=0, \ldots, L,
$$

das Problem 5.1. 
Beweis: Nachzuweisen ist die Gültigkeit der Randbedingungen. Aus $\mathcal{R}_{T, \Gamma_{j}, 1}\left(u_{v(j)},-u_{j}\right)=0, j=1, \ldots, L$ folgt nach Voraussetzung $\left.u_{v(j)}\right|_{\Omega_{j, i}}=0$ und $\left.u_{j}\right|_{\Omega_{j, a}}=0$ und daher

$$
\begin{aligned}
\mathcal{R}_{C, \Gamma_{j}, i} u_{j} & =\mathcal{R}_{C, \Gamma_{j}, i} u_{j}-\mathcal{R}_{C, \Gamma_{j}, a} u_{j}=I_{\rho_{j}}\left(\varphi_{j}+f_{j}\right) \\
& =I_{\rho_{j}}\left(\mathcal{R}_{C, \Gamma_{j}, a} u_{v(j)}-\mathcal{R}_{C, \Gamma_{j}, i} u_{v(j)}+f_{j}\right)=I_{\rho_{j}}\left(\mathcal{R}_{C, \Gamma_{j}, a} u_{v(j)}+f_{j}\right) .
\end{aligned}
$$

Satz 5.7. Wenn das homogene Transmissionsproblem 5.1 nur trivial lösbar ist und die Bedingungen des obigen Satzes erfüllt sind, dann ist $M \varphi=Q f$ eindeutig lösbar.

Beweis: Da es sich um ein Gleichungssystem zweiter Art handelt, ist der Nachweis der Injektivität von $M$ ausreichend. Sei $\varphi$ eine Lösung des homogenen Systems. Aus dem vorherigen Satz folgt, daß $u$ das homogene Transmissionsproblem löst und somit ergibt sich $\left.u_{j}\right|_{\Omega_{j}}=0$. Wie in dem vorherigen Beweis gilt $\left.u_{j}\right|_{\Omega_{j, a}}=0$ und somit $\varphi_{j}=0$ aus den Sprungbeziehungen.

Nun ist noch die Frage zu klären, wie die Matrizen $M$ und $Q$ für allgemeine Ränder zu initialisieren sind. Ähnlich zu dem Fall beim Potentialansatz ergibt sich folgender Algorithmus für $M$ und $Q$ :

for $(j=1, \ldots, L)$

- $\operatorname{for}(1=1, \ldots, L)$

$$
\begin{aligned}
& \text { - if }(j=1) \quad M(j, j)=\mathcal{R}_{T, \Gamma_{j}, 1}\left(\mathcal{G}_{j, a},-\mathcal{G}_{j, i}\right), Q(j, j)=\mathcal{R}_{T, \Gamma_{j}, 1}\left(0, \mathcal{G}_{j, i}\right) \\
& \text { - else if }(\mathrm{v}(j)=\mathrm{v}(1)) \mathrm{M}(j, 1)=\mathcal{R}_{T, \Gamma_{j}, 1}\left(\mathcal{G}_{l, a}, 0\right), Q(j, 1)=0 \\
& \text { - else if }(\mathrm{v}(j)=1) M(j, 1)=\mathcal{R}_{T, \Gamma_{j}, 1}\left(\mathcal{G}_{l, i}, 0\right), Q(j, 1)=\mathcal{R}_{T, \Gamma_{j}, 1}\left(-\mathcal{G}_{l, i}, 0\right) \\
& \text { - else if }(j=\mathrm{v}(1)) M(j, 1)=\mathcal{R}_{T, \Gamma_{j}, 1}\left(0,-\mathcal{G}_{l, a}\right), Q(j, 1)=0 \\
& \text { - else } M(j, 1)=Q(j, 1)=0
\end{aligned}
$$

Für die Implementation dieses Verfahrens kann dies noch etwas anders geschrieben werden:

for $(j=1, \ldots, L)$

- $\operatorname{for}(1=1, \ldots, j-1)$

$$
-\operatorname{if}(\mathrm{v}(j)=\mathrm{v}(1))
$$$$
\text { * } \mathrm{M}(\mathrm{j}, 1)=\mathcal{R}_{T, \Gamma_{j}, 1}\left(\mathcal{G}_{l, a}, 0\right) \mathrm{M}(1, j)=\mathcal{R}_{T, \Gamma_{l}, 1}\left(\mathcal{G}_{j, a}, 0\right)
$$$$
* Q(j, 1)=Q(1, j)=0
$$

$-\operatorname{if}(v(j)=1)$

$* \mathrm{M}(\mathrm{j}, \mathrm{l})=\mathcal{R}_{T, \Gamma_{j}, 1}\left(\mathcal{G}_{l, i}, 0\right) \mathrm{M}(\mathrm{l}, \mathrm{j})=\mathcal{R}_{T, \Gamma_{l}, 1}\left(0,-\mathcal{G}_{j, a}\right)$

* $Q(j, 1)=\mathcal{R}_{T, \Gamma_{j}, 1}\left(-\mathcal{G}_{l, i}, 0\right) \quad Q(1, j)=0$

- else $M(j, I)=M(1, j)=Q(j, 1)=Q(1, j)=0$

- $\mathrm{M}(\mathrm{j}, \mathrm{j})=\mathcal{R}_{T, \Gamma_{j}, 1}\left(\mathcal{G}_{j, a},-\mathcal{G}_{j, i}\right) \quad \mathrm{Q}(\mathrm{j}, \mathrm{j})=\mathcal{R}_{T, \Gamma_{j}, 1}\left(0, \mathcal{G}_{j, i}\right)$ 


\subsection{Ansatz von Kleinman und Martin}

Als letzte Möglichkeit zur Lösung des direkten Problems soll hier ein von Kleinman und Martin entwickelter Ansatz betrachtet werden (vgl. [34] für den Fall einer Randkurve). Bei diesem Ansatz wird pro Rand nur eine (skalare) Dichte benötigt. Allerdings müssen dafür Operatoren hintereinander ausgewertet werden. Dies entspricht bei der numerischen Realisierung dann der Notwendigkeit von Matrizenmultiplikationen. Um die Darstellung möglichst übersichtlich zu halten, werden wir nur die einfachste Variante des Ansatzes vorstellen. Für andere Varianten verweisen wir auf den eben genannten Artikel.

Wir verwenden die Funktion $b$, die abwechselnd die Werte 0 und 1 annimmt:

$$
b:\{0, \ldots, L\} \rightarrow\{0,1\} \text { mit } b(0)=0 \text { und } b(j)=\left\{\begin{array}{ll}
1 & \text { falls } b(v(j))=0, \\
0 & \text { falls } b(v(j))=1
\end{array}, j=1, \ldots, L .\right.
$$

In der Abbildung 5.3 ist eine Beispielgeometrie mit den entsprechenden Werten für die Funktion $b$ dargestellt. Die Idee besteht nun darin, im Außengebiet einen Potentialansatz zu verwenden (wir benutzen hier nur ein Einfachschichtpotential, im allgemeinen Fall würde man ein gemischtes Potential verwenden) und dann abwechselnd Greensche Ansatzfunktionen und Potentialansätze zu benutzen. Sei also $j \in\{0, \ldots, L\}$, dann werden folgende Ansatzfunktionen gewählt:

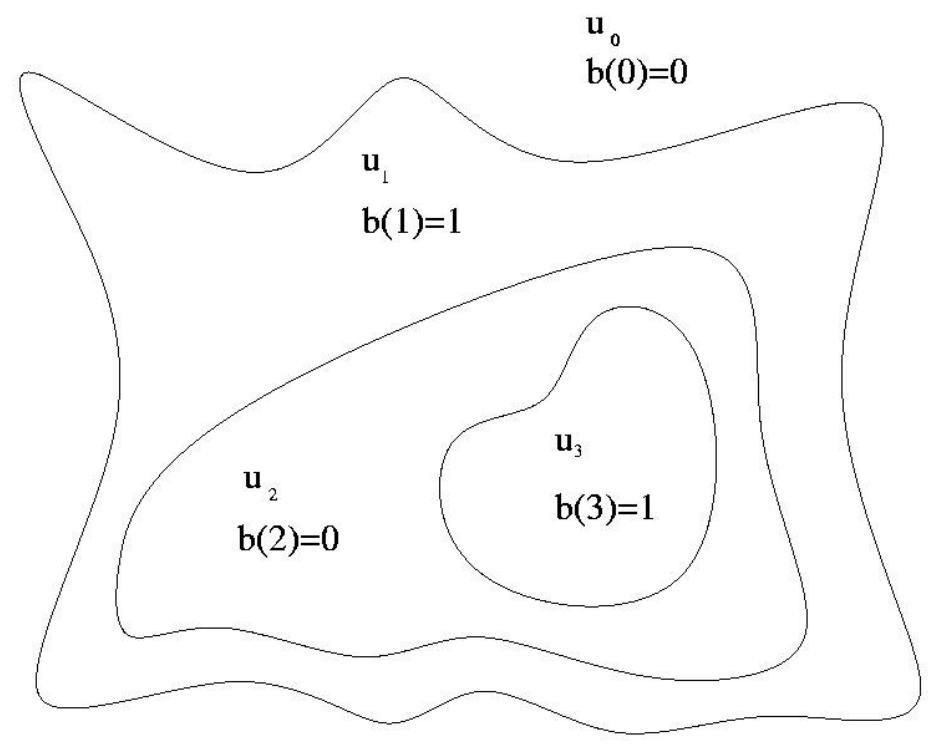

Abbildung 5.3: Beispielgeometrie

$u_{j}:=\left\{\begin{array}{lr}\sum_{l \in n(j)} \mathcal{S}_{l, a} \varphi_{l}+\mathcal{S}_{j, i} \varphi_{j}, & b(j)=0 \\ \sum_{l \in n(j)} \mathcal{G}_{l, a}\left(I_{1 / \rho_{l}} \mathcal{R}_{C, \Gamma_{l}, i} u_{l}-f_{l}\right)+\mathcal{G}_{j, i}\left(\mathcal{R}_{C, \Gamma_{j}, a} u_{v(j)}+f_{j}\right), & b(j)=1\end{array}\right.$

Wie beim Potentialansatz gelte auch hier $\mathcal{S}_{0, i} \varphi_{0}:=0$. Für die Dichten gelte $\varphi_{j} \in$ 
$C^{m-1, \alpha}\left(\Gamma_{j}\right)$. Falls $b(j)=1$, so ergibt sich folgende Darstellung für $u_{j}$ :

$$
\begin{aligned}
u_{j}= & \sum_{l \in n(j)} \mathcal{G}_{l, a} I_{1 / \rho_{l}} \mathcal{R}_{C, \Gamma_{l}, i}\left(\sum_{p \in n(l)} \mathcal{S}_{p, a} \varphi_{p}+\mathcal{S}_{l, i} \varphi_{l}\right)-\sum_{l \in n(j)} \mathcal{G}_{l, a} f_{l} \\
& +\mathcal{G}_{j, i} \mathcal{R}_{C, \Gamma_{j}, a}\left(\sum_{l \in n(v(j))} \mathcal{S}_{l, a} \varphi_{l}+\mathcal{S}_{v(j), i} \varphi_{v(j)}\right)+\mathcal{G}_{j, i} f_{j} \\
= & \sum_{l \in n^{2}(j)} \mathcal{G}_{v(l), a} I_{1 / \rho_{v(l)}} \mathcal{R}_{C, \Gamma_{v(l)}, \mathcal{S}_{l, a}} \mathcal{S}_{l}+\sum_{l \in n(j)} \mathcal{G}_{l, a} I_{1 / \rho_{l}} \mathcal{R}_{C, \Gamma_{l}, i} \mathcal{S}_{l, i} \varphi_{l}-\sum_{l \in n(j)} \mathcal{G}_{l, a} f_{l} \\
& +\sum_{l \in n(v(j))} \mathcal{G}_{j, i} \mathcal{R}_{C, \Gamma_{j}, a} \mathcal{S}_{l, a} \varphi_{l}+\mathcal{G}_{j, i} \mathcal{R}_{C, \Gamma_{j}, a} \mathcal{S}_{v(j), i} \varphi_{v(j)}+\mathcal{G}_{j, i} f_{j} .
\end{aligned}
$$

Nun wird für jeden Rand $\Gamma_{j}$ eine Dirichletrandbedingung vorgegeben. Die Idee besteht darin, für die Greenschen Ansatzfunktionen $u_{j}$ homogene Dirichletrandwerte bei Annäherung an $\partial \Omega_{j}$ von außen zu fordern. Dies wird durch den Greenschen Darstellungssatz nahegelegt. Falls $b(j)=1$ wird also die Gültigkeit von $\mathcal{R}_{D, \Gamma_{j}, a} u_{j}=0$ gefordert, für $b(j)=0$ fordern wir $\mathcal{R}_{D, \Gamma_{j}, i} u_{v(j)}=0$. Das sich daraus ergebende Gleichungssystem bezeichnen wir mit $M \varphi=Q f$.

Satz 5.8. Angenommen $\varphi$ erfüllt das System $M \varphi=Q f$ und $\kappa_{v(j)}$ ist kein DirichletEigenwert in $\Omega_{j, i}(j=1, \ldots, L)$, dann lösen obige Ansatzfunktionen das Problem 5.1.

Beweis. Sei $j \in\{1, \ldots, L\}$. Für $b(j)=1$ gilt $\mathcal{R}_{D, \Gamma_{j}, a} u_{j}=0$, d.h. $u_{j}$ löst in $\Omega_{j, a}$ ein homogenes Dirichletproblem und verschwindet daher identisch. Somit gilt $\mathcal{R}_{C, \Gamma_{j}, a} u_{j}=0$ und aus den Sprungbeziehungen ergibt sich

$$
\begin{aligned}
\mathcal{R}_{C, \Gamma_{j}, i} u_{j} & =-\left(\mathcal{R}_{C, \Gamma_{j}, a}-\mathcal{R}_{C, \Gamma_{j}, i}\right) u_{j} \\
& =-\left(\mathcal{R}_{C, \Gamma_{j}, a}-\mathcal{R}_{C, \Gamma_{j}, i}\right) \mathcal{G}_{j, i}\left(\mathcal{R}_{C, \Gamma_{j}, a} u_{v(j)}+f_{j}\right) \\
& =I_{\rho_{j}}\left(\mathcal{R}_{C, \Gamma_{j}, a} u_{v(j)}+f_{j}\right) .
\end{aligned}
$$

Für $b(j)=0$ gilt $\mathcal{R}_{D, \Gamma_{j}, i} u_{v(j)}=0$, d.h. $u_{v(j)}$ löst in $\Omega_{j, i}$ ein homogenes Dirichletproblem und nach Voraussetzung verschwindet also $u_{v(j)}$ in $\Omega_{j, i}$, d.h. $\mathcal{R}_{C, \Gamma_{j}, i} u_{v(j)}=0$ und daher

$$
\begin{aligned}
\mathcal{R}_{C, \Gamma_{j}, a} u_{v(j)} & =\left(\mathcal{R}_{C, \Gamma_{j}, a}-\mathcal{R}_{C, \Gamma_{j}, i}\right) u_{v(j)} \\
& =\left(\mathcal{R}_{C, \Gamma_{j}, a}-\mathcal{R}_{C, \Gamma_{j}, i}\right) \mathcal{G}_{j, a}\left(I_{1 / \rho_{j}} \mathcal{R}_{C, \Gamma_{j}, i} u_{j}-f_{j}\right) \\
& =I_{1 / \rho_{j}} \mathcal{R}_{C, \Gamma_{j}, i} u_{j}-f_{j},
\end{aligned}
$$

d.h. in beiden Fällen ist die Randbedingung erfüllt.

Satz 5.9. Das Gleichungssystem $M \varphi=Q f$ ist eindeutig lösbar, falls das homogene Transmissionsproblem nur die triviale Lösung besitzt und die Voraussetzungen des letzten Satzes erfüllt sind. 
Beweis. Sei $j \in\{1, \ldots, L\}$. Für $b(j)=1$ gilt $\mathcal{R}_{D, \Gamma_{j}, a} u_{j}=0$, d.h. in diesem Fall gilt

$$
\begin{aligned}
M_{j, j} & =\mathcal{R}_{D, \Gamma_{j}, a} \mathcal{G}_{j, i} \mathcal{R}_{C, \Gamma_{j}, a} \mathcal{S}_{j, a} \\
& =\rho_{j} S_{j, i}\left(K_{j, a}^{*}-1 / 2\right)-\left(K_{j, i}+1 / 2\right) S_{j, a}, \\
Q_{j, j} f_{j} & =-\mathcal{R}_{D, \Gamma_{j}, a} \mathcal{G}_{j, i} f_{j} \\
& =\left(K_{j, i}+1 / 2\right) f_{j, 1}-\rho_{j} S_{j, i} f_{j, 2} .
\end{aligned}
$$

Für $b(j)=0$ gilt $\mathcal{R}_{D, \Gamma_{j}, i} u_{v(j)}=0$ und somit ergibt sich folgende Darstellung für den Diagonaloperator:

$$
\begin{aligned}
M_{j, j} & =\mathcal{R}_{D, \Gamma_{j}, i} \mathcal{G}_{j, a} I_{1 / \rho_{j}} \mathcal{R}_{C, \Gamma_{j}, i} \mathcal{S}_{j, i} \\
& =\left(K_{j, a}-1 / 2\right) S_{j, i}-1 / \rho_{j} S_{j, a}\left(K_{j, i}^{*}+1 / 2\right), \\
Q_{j, j} f_{j} & =\mathcal{R}_{D, \Gamma_{j}, i} \mathcal{G}_{j, a} f_{j} \\
& =\left(K_{j, a}-1 / 2\right) f_{j, 1}-S_{j, a} f_{j, 2} .
\end{aligned}
$$

Somit kann das Gleichungssystem durch Regularisierung mit dem T-Operator (d.h. mit Hilfe der Gleichung $T S=K^{* 2}-I$, vgl. z.B. [7], Gleichung (3.13)) in ein System zweiter Art überführt werden. Es reicht also der Nachweis, daß das homogene System nur trivial lösbar ist. Sei nun $\varphi$ eine Lösung des homogenen Systems, dann lösen die Ansatzfunktionen nach obigem Satz das homogene Problem. Es gilt daher $\left.u_{j}\right|_{\Omega_{j}}=0$ für $j \in\{0, \ldots, L\}$. Sei nun $j \in\{0, \ldots, L\}$ mit $b(j)=0$, dann folgt aus der Stetigkeit des Einfachschichtpotentials in ganz $\mathbb{R}^{d}$, daß die Funktion $u_{j}$ in $\mathbb{R}^{d} \backslash \Omega_{j}$ ein homogenes Dirichletproblem löst und somit gilt $u_{j}{\mid \mathbb{R}^{d} \backslash \Omega_{j}}=0$ und daher folgt aus den Sprungbeziehungen $\varphi_{j}=0$ und $\varphi_{l}=0$ für $l \in n(j)$.

Nun muß noch eine allgemeine Vorschrift zum Initialisieren der Operatormatrix angegeben werden. Dies ist in diesem Fall leider erheblich komplizierter.

for $(j=1, \ldots, n)$

- $\operatorname{for}(1=1, \ldots, n)$

$$
\begin{aligned}
& \text { - if }(\mathrm{b}(\mathrm{j})=1) / / \mathrm{d} . \mathrm{h} \text {. Randbedingung } \mathcal{R}_{D, \Gamma_{j}, a} u_{j}=0 \\
& \text { * if }(j=1) \\
& \mathrm{M}(\mathrm{j}, \mathrm{j})=\mathcal{R}_{D, \Gamma_{j}, a} \mathcal{G}_{j, i} \mathcal{R}_{C, \Gamma_{j}, a} \mathcal{S}_{j, a}, \mathrm{Q}(\mathrm{j}, \mathrm{j})=-\mathcal{R}_{D, \Gamma_{j}, a} \mathcal{G}_{j, i} \\
& * \text { else if }(\mathrm{v}(j)=\mathrm{v}(\mathrm{l})) \\
& \mathrm{M}(\mathrm{j}, \mathrm{I})=\mathcal{R}_{D, \Gamma_{j}, a} \mathcal{G}_{j, i} \mathcal{R}_{C, \Gamma_{j}, a} \mathcal{S}_{l, a}, \mathrm{Q}(\mathrm{j}, \mathrm{l})=0 \\
& \text { * else if }(\mathrm{v}(j)=1) \\
& \mathrm{M}(\mathrm{j}, \mathrm{l})=\mathcal{R}_{D, \Gamma_{j}, a} \mathcal{G}_{j, i} \mathcal{R}_{C, \Gamma_{j}, a} \mathcal{S}_{l, i}, \mathrm{Q}(\mathrm{j}, \mathrm{l})=0 \\
& * \text { else if }(j=v(1)) \\
& \mathrm{M}(\mathrm{j}, \mathrm{l})=\mathcal{R}_{D, \Gamma_{j}, a} \mathcal{G}_{l, a} I_{1 / \rho_{l}} \mathcal{R}_{C, \Gamma_{l}, i} \mathcal{S}_{l, i}, \quad \mathrm{Q}(\mathrm{j}, \mathrm{l})=\mathcal{R}_{D, \Gamma_{j}, a} \mathcal{G}_{l, a} \\
& \text { * else if }(j=\mathrm{v}(\mathrm{v}(1))) \\
& \mathrm{M}(\mathrm{j}, \mathrm{l})=\mathcal{R}_{D, \Gamma_{j}, a} \mathcal{G}_{v(l), a} I_{1 / \rho_{v(l)}} \mathcal{R}_{C, \Gamma_{v(l)}, i} \mathcal{S}_{l, a}, \mathrm{Q}(\mathrm{j}, \mathrm{l})=0 \\
& \text { * else } M(j, 1)=Q(j, 1)=0
\end{aligned}
$$




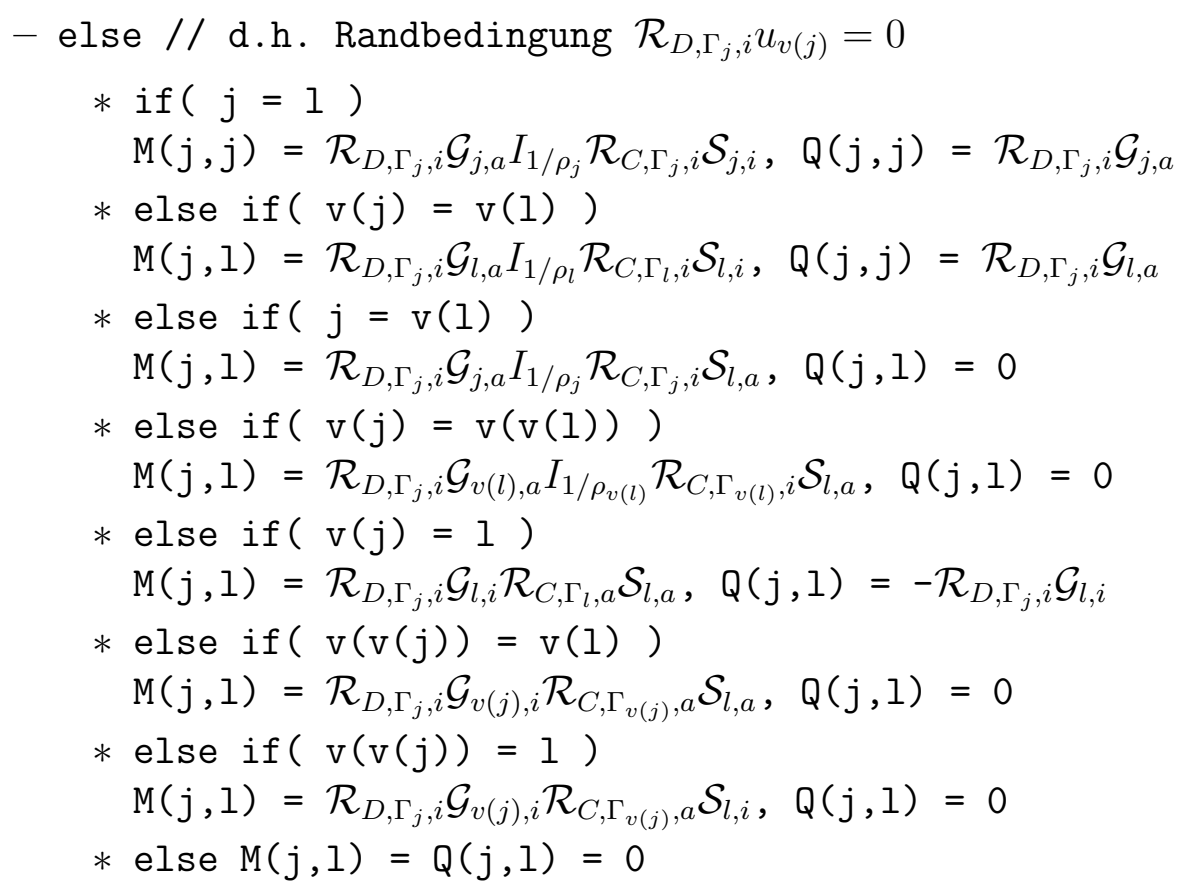

Auch wenn das Verfahren von Kleinman-Martin aus theoretischer Sicht sehr interessant ist, da nur eine skalare Dichte pro Rand benötigt wird, ist die Implementation für mehrere Gebiete sehr aufwendig. Wir haben dieses Verfahren daher nur für eine einzige Randkurve getestet und uns bei mehreren Kurven auf den Potential-, und den Greenschen Ansatz beschränkt.

\subsection{Inhomogenes Transmissionsproblem}

Zum Ende dieses Kapitels werden die vorherigen Ergebnisse noch einmal in Operatornotation zusammengefaßt und gleichzeitig behandeln wir das Transmissionsproblem zur inhomogenen Helmholtzgleichung.

Satz 5.10. Sei das homogene Problem 5.1 eindeutig lösbar. Dann existiert für $g \in$ $C_{0}^{m-2, \alpha}$ und $f \in C^{m, m-1, \alpha}(\partial \Omega)$ genau ein $u \in \hat{C}^{m, \alpha}$ mit

$$
\left(\mathcal{L}_{T}, \mathcal{R}_{T}\right) u=(g, f) .
$$

Für den Operator $\mathcal{D}_{T}:(g, f) \mapsto u$ gilt $\mathcal{D}_{T} \in L\left(C_{0}^{m-2, \alpha} \times C^{m, m-1, \alpha}(\Gamma), \hat{C}^{m, \alpha}\right)$. Weiterhin ist $\mathcal{D}_{T}$ invertierbar mit $\mathcal{D}_{T}^{-1}=\left(\mathcal{L}_{T}, \mathcal{R}_{T}\right)$.

Beweis. Die Existenz und Eindeutigkeit des Operators $\mathcal{D}_{T}^{h} \in L\left(C^{m, m-1, \alpha}(\Gamma), \hat{C}^{m, \alpha}\right)$ mit $\left(\mathcal{L}_{T}, \mathcal{R}_{T}\right) \mathcal{D}_{T}^{h}=(0, I)$ wurde bereits bewiesen. Somit erfüllt $\mathcal{D}_{T}$ mit

$$
\mathcal{D}_{T}(g, f):=-\left[V_{\Omega_{j}} g_{j}\right]_{j=0}^{L}+\mathcal{D}_{T}^{h}\left(f+\mathcal{R}_{T}\left[V_{\Omega_{j}} g_{j}\right]_{j=0}^{L}\right)
$$

die Forderungen des Satzes. 


\section{Analytische Abhängigkeit von den Problemdaten}

In diesem Kapitel wird die analytische Abhängigkeit von Lösungen des Dirichlet-, Neumann-, Robin-- und des Transmissionsproblems bzgl. des Randes, der Wellenzahl und der Übergangskonstanten $\rho$ (beim Transmissionsproblem) untersucht. Sei für das gesamte Folgende $m \in \mathbb{N}_{\geq 2}, 0<\alpha<1$ und $\Omega \subset \mathbb{R}^{d}, d=2,3$, ein $C^{m, \alpha_{\text {-Gebiet mit }}}$ beschränktem Rand. Sei $B_{R}$ eine Kugel um den Nullpunkt mit dem Radius $R>0$, die den Rand $\partial \Omega$ echt enthält, das Gebiet $\Omega$ sei also von der in Kapitel 2 beschriebenen Form. Als Randwerte verwenden wir nun stets eine einfallende ebene Welle

$$
u_{i}(x):=e^{i \kappa x \cdot v}, \quad v \in S^{d-1},
$$

mit Einfallsrichtung $v$. D.h. wir wählen $-\mathcal{R}_{D} u_{i}$ als Randwerte beim Dirichlet-Problem und $-\mathcal{R}_{N} u_{i}$ beim Neumann-Problem. Wir werden im folgenden hauptsächlich das Dirichlet- und das Neumann-Problem betrachten, da die Beweise für die anderen beiden Randwertprobleme analog verlaufen.

Mit $u$ bezeichnen wir die Lösung des Randwertproblems und im Fall eines unbeschränkten Gebietes $\Omega$ mit $u_{\infty}$ das Fernfeld der Lösung $u$. Die Randwerte sind gerade so gewählt, daß $\mathcal{R}_{D / N}\left(u+u_{i}\right)=0$ gilt. Zunächst soll der Begriff der Analytizität in Banachräumen erklärt werden.

\subsection{Analytische Abbildungen}

Definition 6.1. Seien $X, Y$ und $Z$ Banach-Räume. Die Abbildung $f: X \rightarrow Z$ heißt analytisch in $x_{0} \in X$, falls $f$ beliebig oft in $x_{0}$ Fréchet-differenzierbar ist und ein $r>0$ existiert mit

$$
\sum_{n=0}^{\infty} \frac{1}{n !}\left\|f^{(n)}\left[x_{0}\right]\right\| r^{n}<\infty \text { und } f\left[x_{0}+h\right]=\sum_{n=0}^{\infty} \frac{1}{n !} f^{(n)}\left[x_{0} ; h^{n}\right]
$$

für alle $h \in X$ mit $\|h\| \leq r$ (hierbei ist $f^{(n)}$ die n-te Fréchet-Ableitung von $f$ und $\left.f^{(n)}\left[x_{0} ; h^{n}\right]:=f^{(n)}\left[x_{0} ; h, \ldots, h\right]\right)$. Weiterhin heißt $f$ analytisch in der offenen Menge $W \subset X$, falls $f$ in jedem Punkt von $W$ analytisch ist.

Die Abbildung $f: X \times Y \rightarrow Z$ heißt analytisch in $\left(x_{0}, y_{0}\right) \in X \times Y$, falls $f$ in $\left(x_{0}, y_{0}\right)$ beliebig oft Fréchet-differenzierbar ist und ein $r>0$ existiert mit

$$
\sum_{n=0}^{\infty} \sum_{i+j=n} \frac{1}{i ! j !}\left\|f^{(i j)}\left[x_{0}, y_{0}\right]\right\| r^{n}<\infty \text { und }
$$




$$
f\left[x_{0}+h_{x}, y_{0}+h_{y}\right]=\sum_{n=0}^{\infty} \sum_{i+j=n} \frac{1}{i ! j !} f^{(i j)}\left[x_{0}, y_{0} ; h_{x}^{i}, h_{y}^{j}\right]
$$

für alle $h=\left(h_{x}, h_{y}\right) \in X \times Y$ mit $\|h\| \leq r$ (hierbei ist $f^{(i j)}$ die partielle FréchetAbleitung von $f$, bei der $i$ mal nach der ersten Variablen und $j$ mal nach der zweiten Variablen differenziert wird). Auch hier heißt $f$ analytisch in der offenen Menge $W \subset$ $X \times Y$, falls $f$ in jedem Punkt von $W$ analytisch ist.

Lemma 6.2. 1. Seien $X, Y$ Banach-Räume, $Z$ eine Banach-Algebra und $f: X \rightarrow$ $Z$ und $g: Y \rightarrow Z$ analytisch. Dann ist auch $w: X \times Y \rightarrow Z$ mit $w(x, y):=$ $f(x) g(y)$ analytisch.

2. Seien $X, Y$ und $Z$ Banach-Räume über dem gleichen Körper $\mathbb{R}$ oder $\mathbb{C}, f: X \rightarrow$ $Y$ und $g: Y \rightarrow Z$ analytisch. Dann ist auch $g \circ f: X \rightarrow Z$ analytisch.

Beweis. Eine einfache Rechnung ergibt zunächst $w^{(i j)}=f^{(i)} g^{(j)}$. Die erste Behauptung folgt dann wie in dem Standardsatz über das Cauchy-Produkt von absolut konvergenten Reihen (vgl. z.B. [12], Seite 47, Satz 3).

Nun zur zweiten Behauptung. Falls die Räume komplexe Banach-Räume sind, ist die Analytizität äquivalent zur komplexen Differenzierbarkeit (vgl. [2], Theorem 2.3.3, Seite 84f.), d.h. die Aussage folgt aus der Kettenregel. Falls $X$ und $Y$ reell sind, betrachtet man Komplexifizierungen $X_{\mathbb{C}}$ und $Y_{\mathbb{C}}$ von $X$ und $Y$ (vgl. [56], Kapitel 1.3) und die analytischen Fortsetzungen der Funktionen (vgl. [9], Seite 151, Beweis von Satz 15.3) und argumentiert wie davor.

Von zentraler Bedeutung ist eine Version des Satzes über implizite Funktionen für analytische Abbildungen:

Satz 6.3. Seien $X, Y, Z$ Banach-Räume über dem gleichen Körper $\mathbb{R}$ oder $\mathbb{C}$ und $W \subset X$ und $V \subset Y$ Umgebungen von $x_{0} \in X$ und $y_{0} \in Y$. Die Abbildung $F$ : $W \times V \rightarrow Z$ sei analytisch und es gelte $F\left[x_{0}, y_{0}\right]=0$ und $F_{y}^{-1}\left[x_{0}, y_{0}\right] \in L(Z, Y)$. Dann existieren Umgebungen $B_{r}\left(x_{0}\right) \subset W$ und $B_{\delta}\left(y_{0}\right) \subset V$, so daß es genau eine Abbildung $T: B_{r}\left(x_{0}\right) \rightarrow B_{\delta}\left(y_{0}\right)$ mit $T x_{0}=y_{0}$ und $F(x, T x)=0$ in $B_{r}\left(x_{0}\right)$ gibt. Diese Abbildung ist analytisch.

Beweis. Vgl. Theorem 15.1 und Theorem 15.3 in [9].

\subsection{Ableitung nach dem Rand}

In diesem Abschnitt wollen wir die Differenzierbarkeit von Lösungen der Randwertprobleme bzgl. des Randes untersuchen. Sei $\vartheta \in C^{m, \alpha}\left(\partial \Omega, \mathbb{R}^{d}\right)$ eine hinreichend kleine Störung von $\partial \Omega$. Das durch $(I+\vartheta)(\partial \Omega)$ berandete Gebiet nennen wir $\Omega_{\vartheta}$. Mit $u[\vartheta]$ bezeichnen wir die Lösung des Randwertproblems in $\Omega_{\vartheta}$ und mit $u_{\infty}[\vartheta]$ das zugehörige Fernfeld (bei unbeschränktem Gebiet $\Omega$ ). Untersucht man die Differenzierbarkeit von $u[\vartheta]$ bzgl. der Störung $\vartheta$, so ergibt sich das Problem, daß der Definitionsbereich von 
$u[\vartheta]$ von $\vartheta$ abhängt. Wie in der Dissertation von Hohage (vgl. [27]) setzen wir daher $\vartheta \mathrm{zu}$ einem Vektorfeld $\theta:=\mathbb{R}^{d} \rightarrow \mathbb{R}^{d}$ fort und betrachten Störungen $I_{\theta}:=I+\theta$ von $\Omega$, die wir mit $\Omega_{\theta}$ bezeichnen (vgl. die Abbildung 6.1). Als offene Teilmenge der $C^{m, \alpha}-$ Vektorfelder betrachten wir die Menge

$$
W:=\left\{\theta \in C^{m, \alpha}\left(\mathbb{R}^{d}, \mathbb{R}^{d}\right): \operatorname{supp}(\theta) \subset B_{R},\|\theta\|_{m, \alpha}<\frac{1}{2 d},(I+\theta)(\Gamma) \in C^{m, \alpha}\right\} .
$$

Hierbei ist die Norm auf $C^{m, \alpha}\left(\mathbb{R}^{d}, \mathbb{R}^{d}\right)$ so gewählt, daß $\|\theta\|_{m, \alpha}<1 / 2 d$ stets $\|\nabla \theta\|_{\infty}<1 / 2$ zur Folge hat, d.h. die Vektorfelder aus $W$ sind stets kontrahierende Abbildungen. Weiterhin benötigen wir Teilmengen der $C^{m, \alpha}$-Störungen des Randes $\partial \Omega$ :

$$
\begin{gathered}
V_{n}:=\left\{\vartheta \in C^{m, \alpha}\left(\partial \Omega, \mathbb{R}^{d}\right):\right. \\
\left.E_{n, \partial \Omega} \vartheta \in W\right\},
\end{gathered}
$$

wobei $E_{n, \partial \Omega}$ der in Lemma 2.2 definierte Fortsetzungsoperator ist. Nun wollen wir die wichtigsten Schritte beim Nachweis der Differenzierbarkeit von $u[\vartheta]$ zunächst ohne Beweis darstellen. Hierzu orientieren wir uns an der Abbildung 6.2 .

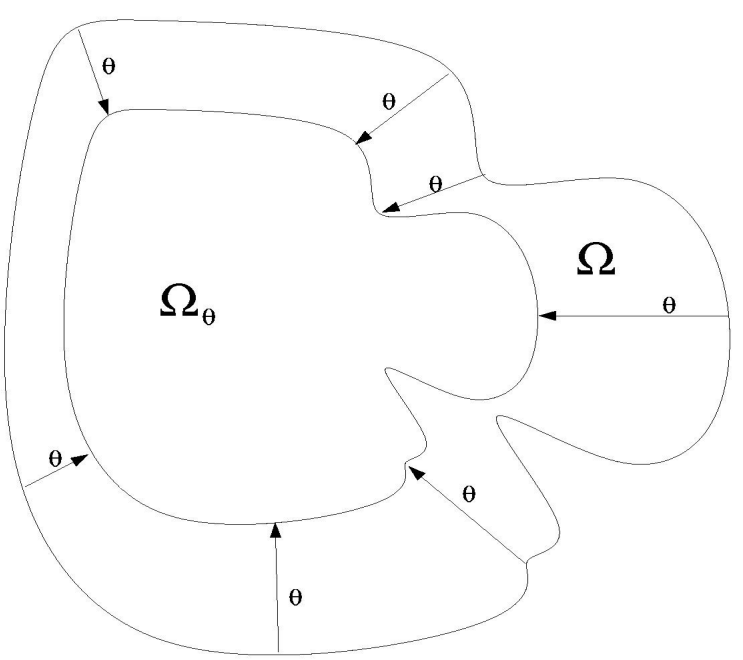

Abbildung 6.1: Störung des Gebietes

1. Zunächst greifen wir eine bereits von Simon (vgl. [58]) benutzte Idee auf und betrachten die Abbildung $\tilde{u}[\theta]:=$ $u\left[\left.\theta\right|_{\partial \Omega}\right] \circ I_{\theta}$. Mit Hilfe des impliziten Funktionentheorems kann hier die Analytizität von $\tilde{u}$ nachgewiesen werden. Entscheidend hierbei ist die beschränkte Invertierbarkeit der Lösungsoperatoren für die betrachteten inhomogenen Randwertprobleme.

2. Sei $\Omega^{\prime} \Subset \Omega$ und $l \in \mathbb{N}$, dann gilt $u[\vartheta]=\tilde{u}\left[E_{n, \partial \Omega} \vartheta\right]$ für $\vartheta \in V_{n}$ und hinreichend großes $n \in \mathbb{N}$. Diese Gleichheit ist der Grund für die Verwendung von Fortsetzungen des Randes. Würden wir nur mit Störungen des Gebietes $\Omega$ arbeiten, so würde diese Gleichheit im Allgemeinen nicht gelten. Aus Satz 3.1, Punkt 3, folgt nun die Analytizität von $u: V_{n} \rightarrow C^{l}\left(\overline{\Omega^{\prime}}\right)$. Mit Hilfe des Greenschen Darstellungssatzes zeigt man, daß alle Ableitungen von $u$ die Helmholtzgleichung erfüllen und daß bei unbeschränktem $\Omega u_{\infty}$ analytisch ist mit $u_{\infty}^{(j)}\left[0 ; h^{j}\right]=\left(u^{(j)}\left[0 ; h^{j}\right]_{B_{R, e}}\right)_{\infty}$.

3. Um nun das Problem mit den unterschiedlichen Definitionsbereichen von $u[\vartheta]$ in den Griff zu bekommen, setzen wir $\tilde{u}$ mit Hilfe des Fortsetzungsoperators $E_{\Omega}$ auf ganz $\mathbb{R}^{d}$ fort. Aus der Linearität des Fortsetzungsoperators folgt dann die Analytizität von $\tilde{U}: W \rightarrow C^{m, \alpha}\left(\mathbb{R}^{d}\right)$ mit $\tilde{U}[\theta]:=E_{\Omega} \tilde{u}[\theta]$. 
4. Jetzt betrachten wir $\check{U}: W \rightarrow C^{m-j, \alpha^{\prime}}\left(\mathbb{R}^{d}\right)$ mit $\check{U}[\theta]:=\tilde{U}[\theta] \circ I_{\theta}^{-1}$ und $0<$ $\alpha^{\prime}<\alpha$. Es gilt also $\left.\check{U}[\theta]\right|_{\Omega_{\theta}}=u\left[\left.\theta\right|_{\partial \Omega}\right]$ und somit stellt $\check{U}[\theta]$ gerade eine geeignete Fortsetzung von $u[\theta]$ dar. Eine Anwendung der Taylorschen Formel ergibt, daß $\check{U} j$-mal differenzierbar $(j=0, \ldots, m)$ ist.

5. Nun sind wir fast am Ziel und müssen nur noch alle Ergebnisse zusammensetzen. Die Abbildungen $U_{n}: V_{n} \rightarrow C^{m-j, \alpha^{\prime}}\left(\mathbb{R}^{d}\right)$ mit $U_{n}[\vartheta]=\check{U}\left[E_{n, \partial \Omega} \theta\right]$ sind j-mal differenzierbar $(j=0, \ldots, m)$; für $h \in C^{m, \alpha}\left(\partial \Omega, \mathbb{R}^{d}\right)$ ist $U^{(j)}\left[0 ; h^{j}\right]:=\left.U_{n}^{(j)}\left[0 ; h^{j}\right]\right|_{\bar{\Omega}}$ unabhängig von $n \in \mathbb{N}$ und für alle $\Omega^{\prime} \Subset \Omega$ gilt $\left.U^{(j)}\left[0 ; h^{j}\right]\right|_{\Omega^{\prime}}=\left.u^{(j)}\left[0 ; h^{j}\right]\right|_{\Omega^{\prime}}$. Weiterhin ergibt sich:

a) $\left.\left(\Delta+\kappa^{2}\right) U^{(j)}\left[0 ; h^{j}\right]\right|_{\Omega}=0$

b) $u_{\infty}^{(j)}\left[0 ; h^{j}\right]=\left(U^{(j)}\left[0 ; h^{j}\right]\right)_{\infty}$ bei unbeschränktem $\Omega$

c) $\left.U^{(j)}\left[0 ; h^{j}\right]\right|_{\partial \Omega}=-\nabla^{j}\left(u_{i}+u[0]\right) \cdot h^{j}-\sum_{l=1}^{j}\left(\begin{array}{l}j \\ l\end{array}\right) \nabla^{l} U^{(j-l)}\left[0 ; h^{j-l}\right] \cdot h^{l}$

Nach dieser Übersicht betrachten wir die Aussagen nun im Detail.

Lemma 6.4. Sei $\theta$ aus $W$, dann ist $I_{\theta}$ ein $C^{m, \alpha}$-Diffeomorphismus.

Beweis. Sei $I_{\theta}(x)=I_{\theta}(y)$, dann folgt $|x-y|=|\theta(x)-\theta(y)| \leq \frac{1}{2}|x-y|$ und somit $x=y$, d.h. $I_{\theta}$ ist injektiv. Sei nun $y \in \mathbb{R}^{d}$ beliebig und definiere $g(x):=y-\theta(x)$, dann ist $g$ eine Kontraktion auf $\mathbb{R}^{d}$ und somit existiert genau ein $x \in \mathbb{R}^{d}$ mit $g(x)=x$, d.h. $I_{\theta}(x)=y$. Somit ist $I_{\theta}$ surjektiv. Weiterhin ist die Funktionalmatrix $\nabla I_{\theta}(x)=$ $\mathbb{1}+\nabla \theta(x)$ invertierbar nach dem Satz über die Neumannsche Reihe (11 bezeichnet hierbei die Identitätsmatrix), denn es gilt $\|\nabla \theta\| \leq \frac{1}{2}$. Somit existiert $\nabla I_{\theta}^{-1}$ und es gilt

$$
\nabla I_{\theta}^{-1}=\left(\nabla I_{\theta} \circ I_{\theta}^{-1}\right)^{-1}
$$

(vgl. z.B. [13], Seite 75, Satz 3). Hieraus folgt die Differenzierbarkeit von $\nabla I_{\theta}^{-1}$ mit

$$
\begin{aligned}
\nabla^{2} I_{\theta}^{-1} & =-\left(\nabla I_{\theta} \circ I_{\theta}^{-1}\right)^{-1} \cdot \nabla^{2} I_{\theta} \circ I_{\theta}^{-1} \cdot \nabla I_{\theta}^{-1} \cdot\left(\nabla I_{\theta} \circ I_{\theta}^{-1}\right)^{-1} \text { bzw. } \\
\nabla^{2} I_{\theta}^{-1} \circ I_{\theta} & =-\left(\nabla I_{\theta}\right)^{-1} \cdot \nabla^{2} I_{\theta} \cdot\left(\nabla I_{\theta}\right)^{-1} \cdot\left(\nabla I_{\theta}\right)^{-1} .
\end{aligned}
$$

Aus dieser Gleichung folgt somit die Hölderstetigkeit von $\nabla^{2} I_{\theta}^{-1}$, denn $\nabla I_{\theta}^{-1}$ ist differenzierbar und somit hölderstetig, ebenso $\left(\nabla I_{\theta}\right)^{-1}$ und somit auch $\left(\left(\nabla I_{\theta}\right) \circ I_{\theta}^{-1}\right)^{-1}$ und auch das Produkt von hölderstetigen Funktionen ist wieder hölderstetig. Analog kann man Formeln für die höheren Ableitungen herleiten.

Betrachte nun die Abbildungen

$$
\begin{aligned}
\mathcal{L}: W \times C^{m, \alpha}(\bar{\Omega}) & \rightarrow C^{m-2, \alpha}(\bar{\Omega}), & \mathcal{L}[\theta, v] & =\Delta_{\theta} v+\kappa^{2} v \\
\mathcal{R}_{D}: W \times C^{m, \alpha}(\bar{\Omega}) & \rightarrow C^{m, \alpha}(\partial \Omega), & \mathcal{R}_{D}[\theta, v] & =v, \\
\mathcal{R}_{N}: W \times C^{m, \alpha}(\bar{\Omega}) & \rightarrow C^{m-1, \alpha}(\partial \Omega), & \mathcal{R}_{N}[\theta, v] & =\nabla v \cdot\left(\nabla I_{\theta}\right)^{-1} \cdot\left(\nu[\theta] \circ I_{\theta}\right),
\end{aligned}
$$


Sei $u[\vartheta] \in C^{m, \alpha}\left(\overline{\Omega_{\vartheta}}\right)$ Lösung des Randwertproblems.

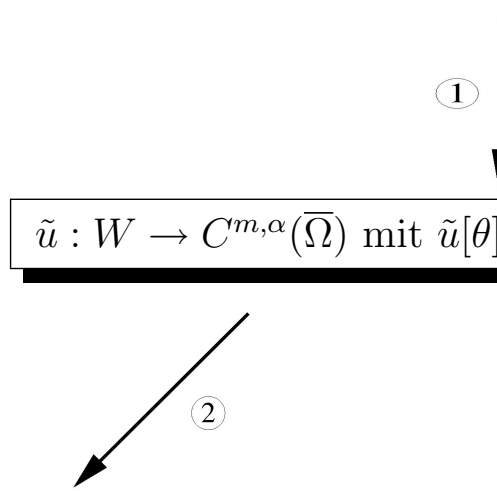

Sei $l \in \mathbb{N}$ und $h \in C^{m, \alpha}\left(\partial \Omega, \mathbb{R}^{d}\right)$ dann existiert für alle $\Omega^{\prime} \Subset \Omega$ ein $n \in \mathbb{N}$, so daß

1. $u: V_{n} \rightarrow C^{l}\left(\overline{\Omega^{\prime}}\right)$ analytisch

2. $\left.\left(\Delta+\kappa^{2}\right) u^{(j)}\left[0 ; h^{j}\right]\right|_{\Omega^{\prime}}=0$

3. $u_{\infty}: V_{1} \rightarrow L^{2}\left(S^{d-1}\right)$ analytisch mit $u_{\infty}^{(j)}\left[0 ; h^{j}\right]=$ $\left(\left.u^{(j)}\left[0 ; h^{j}\right]\right|_{B_{R, e}}\right)_{\infty}$
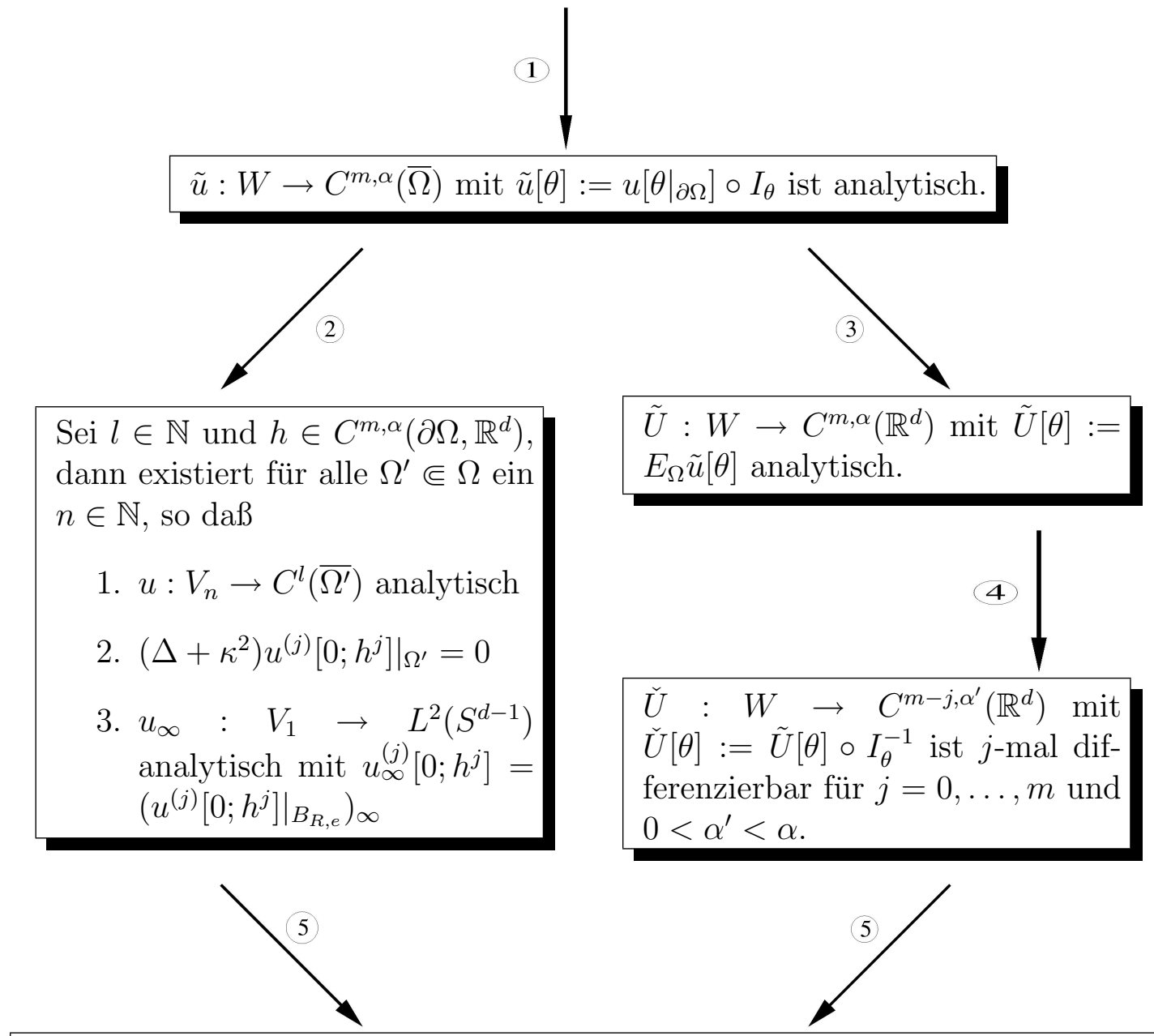

$U_{n}: V_{n} \rightarrow C^{m-j, \alpha^{\prime}}\left(\mathbb{R}^{d}\right)$ mit $U_{n}[\vartheta]=\check{U}\left[E_{n, \partial \Omega} \theta\right]$ ist j-mal differenzierbar $(j=$ $0, \ldots, m)$; für $h \in C^{m, \alpha}\left(\partial \Omega, \mathbb{R}^{d}\right)$ ist $U^{(j)}\left[0 ; h^{j}\right]:=\left.U_{n}^{(j)}\left[0 ; h^{j}\right]\right|_{\bar{\Omega}}$ unabhängig von $n \in \mathbb{N}$ und für alle $\Omega^{\prime} \Subset \Omega$ gilt $\left.U^{(j)}\left[0 ; h^{j}\right]\right|_{\Omega^{\prime}}=\left.u^{(j)}\left[0 ; h^{j}\right]\right|_{\Omega^{\prime}}$. Weiterhin ergibt sich:

1. $\left.\left(\Delta+\kappa^{2}\right) U^{(j)}\left[0 ; h^{j}\right]\right|_{\Omega}=0$

2. $u_{\infty}^{(j)}\left[0 ; h^{j}\right]=\left(U^{(j)}\left[0 ; h^{j}\right]\right)_{\infty}$ bei unbeschränktem $\Omega$.

3. $\left.U^{(j)}\left[0 ; h^{j}\right]\right|_{\partial \Omega}=-\nabla^{j}\left(u_{i}+u[0]\right) \cdot h^{j}-\sum_{l=1}^{j}\left(\begin{array}{l}j \\ l\end{array}\right) \nabla^{l} U^{(j-l)}\left[0 ; h^{j-l}\right] \cdot h^{l}$

Abbildung 6.2: Struktur des Beweises 
$\mathcal{R}_{N}$ wurde gerade so definiert, daß $\mathcal{R}_{N}[\theta, \tilde{u}[\theta]]=\frac{\partial u[\theta \mid \partial \Omega]}{\partial \nu[\theta]} \circ I_{\theta}$ gilt (hierbei ist $\nu[\theta]$ der Normalenvektor in $\Omega_{\theta}$ ). Zudem gilt $\mathcal{R}_{D}[0, v]=\mathcal{R}_{D} v$ und ebenso $\mathcal{R}_{N}[0, v]=\mathcal{R}_{N} v$ mit den in Kapitel 4 definierten Operatoren $\mathcal{R}_{D}$ und $\mathcal{R}_{N}$. Weiterhin ist $\Delta_{\theta}$ gegeben durch

$$
\begin{aligned}
\Delta_{\theta} v & =\sum_{k, l} g_{\theta}^{k, l} \frac{\partial^{2} v}{\partial x_{k} \partial x_{l}}+\sum_{k}\left(\frac{1}{\sqrt{g_{\theta}}} \sum_{l} \frac{\partial\left(g_{\theta}^{k l} \sqrt{g_{\theta}}\right)}{\partial x_{l}}\right) \frac{\partial v}{\partial x_{k}} \\
& =\sum_{k, l} g_{\theta}^{k, l} \frac{\partial^{2} v}{\partial x_{k} \partial x_{l}}+\sum_{k}\left(\sum_{l}\left(\frac{\partial g_{\theta}^{k l}}{\partial x_{l}}+\frac{g_{\theta}^{k l}}{2 g_{\theta}} \frac{\partial g_{\theta}}{\partial x_{l}}\right)\right) \frac{\partial v}{\partial x_{k}}
\end{aligned}
$$

Es sei $G_{\theta}=\left(g_{\theta, k l}\right)=\left(\nabla I_{\theta}\right)^{T} \cdot \nabla I_{\theta}, g_{\theta}=\operatorname{det} G_{\theta},\left(g_{\theta}^{k l}\right)=G_{\theta}^{-1}$ und der transformierte Laplaceoperator ist bekanntlich definiert durch $\Delta_{\theta}\left(v \circ I_{\theta}\right)=(\Delta v) \circ I_{\theta}$ (vgl. z.B. [14], Seite 30).

Lemma 6.5. Die Operatoren $\mathcal{L}, \mathcal{R}_{D}$ und $\mathcal{R}_{N}$ sind analytisch.

Beweis. Zunächst zum Operator $\mathcal{L}$. Die Aussage wird für alle Terme getrennt nachgewiesen, wobei ausgenutzt wird, daß die Multiplikation von analytischen Abbildungen wieder eine analytische Abbildung ergibt (vgl. Satz 6.2). Bzgl. des zweiten Argumentes ist $\mathcal{L}$ linear, d.h. analytisch, so daß nur $\theta \mapsto \mathcal{L}(\theta, v)$ für ein $v \in C^{m, \alpha}(\Omega)$ zu untersuchen ist.

Zunächst ist klar, daß $\theta \mapsto \nabla I_{\theta}$ analytisch ist, da die Abbildung affin in $\theta$ ist. Somit ist auch klar, daß $\theta \mapsto G_{\theta}=\nabla I_{\theta}^{T} \cdot \nabla I_{\theta}$ analytisch ist, da die Multiplikation von analytischen Funktionen analytisch ist. Nun soll gezeigt werden, daß $(\theta, v) \mapsto$ $\left(\nabla I_{\theta}\right)^{-1}=: B[\theta]$ analytisch ist. Zunächst zeigt man durch Induktion die Gültigkeit von

$$
B^{(l)}\left[\theta, h^{l}\right]:=(-1)^{l} l ! \underbrace{B[\theta] \cdot \nabla h \cdot B[\theta] \cdot \nabla h \cdot \ldots \cdot \nabla h \cdot B[\theta]}_{2 l+1-\text { Terme }} .
$$

Nach der Taylorschen Formel (vgl. z.B. [64], S.243) folgt:

$$
B[\theta+h]=\sum_{l=0}^{n-1} \frac{1}{l !} B^{(l)}\left[\theta ; h^{l}\right]+R_{n},
$$

wobei der Restterm $R_{n}$ abgeschätzt werden kann durch

$$
\left\|R_{n}\right\|_{m-2, \alpha}=\left\|\int_{0}^{1} \frac{(1-s)^{n-1}}{(n-1) !} B^{(n)}\left[\theta+s h, h^{n}\right] \mathrm{ds}\right\|_{m-2, \alpha} \leq n\|B[\theta]\|_{m-2, \alpha}^{n+1}\|\nabla h\|_{m-2, \alpha}^{n} .
$$

Es ist $\|B[\theta]\|_{m-2, \alpha} \leq 2$, d.h. für $\|\nabla h\|_{m-2, \alpha} \leq \frac{1}{4}$ gilt $\lim _{n \rightarrow \infty}\left\|R_{n}\right\|_{m-2, \alpha}=0$. Somit gilt $B[\theta+h]=\sum_{l=0}^{\infty} \frac{1}{l !} B^{(l)}\left[\theta ; h^{l}\right]$ und ebenso zeigt man $\sum_{l=0}^{\infty} \frac{1}{l !}\left\|B^{(l)}\left[\theta ; h^{l}\right]\right\|<\infty$ für $h$ hinreichend klein. Somit ist auch die Abbildung $\theta \mapsto G_{\theta}^{-1}=\left(\nabla I_{\theta}\right)^{-1} \cdot\left(\nabla I_{\theta}\right)^{-T}$ und daher auch die Abbildung $\theta \mapsto g_{\theta}^{k l}$ analytisch. 
Die Abbildungen $\theta \mapsto \operatorname{det} G_{\theta}$ und $\theta \mapsto \operatorname{det} G_{\theta}^{-1} \operatorname{sind}$ analytisch, da die Determinante ein Polynom in den Matrixeinträgen ist. Beachte weiterhin, $\operatorname{daß} \frac{1}{g_{\theta}}=\operatorname{det} G_{\theta}^{-1}$ (Determinantenmultiplikationsformel) gilt. $\theta \rightarrow \nabla\left(\nabla I_{\theta}\right)^{-1}=-\left(\nabla I_{\theta}\right)^{-1} \cdot \nabla^{2} I_{\theta} \cdot\left(\nabla I_{\theta}\right)^{-1}$ ist analytisch, da Multiplikation von analytischen Funktionen und somit ist auch $\theta \mapsto \frac{\partial g_{\theta}^{k l}}{\partial x_{l}}$ analytisch.

$\frac{\partial}{\partial x_{l}} \operatorname{det} G_{\theta}=\sum_{\sigma \in S_{n}} \operatorname{sign}(\sigma) \sum_{i=1}^{d} \frac{\partial a_{i \sigma(i)}}{\partial x_{l}} \prod_{j=1, j \neq i}^{d} a_{j \sigma(j)}$ ist ebenfalls analytisch, da es ein Polynom analytischer Funktionen ist, wobei $\left\{a_{i j}\right\}$ die Matrixelemente von $G_{\theta}$ sind.

Nun zu den Randoperatoren. Beide Operatoren sind linear bzgl. des zweiten Argumentes, so daß nur die Abhängigkeit bzgl. des ersten Argumentes zu untersuchen ist. $\mathcal{R}_{D}$ ist konstant bzgl. der ersten Variable, so daß hier nichts zu zeigen ist. Die Analytizität der Abbildung $\theta \rightarrow\left(\nabla I_{\theta}\right)^{-1}$ wurde bereits gezeigt, so daß zum Nachweis der Behauptung für $\mathcal{R}_{N}$ noch die Analytizität von $\theta \rightarrow \nu[\theta] \circ I_{\theta}$ zu zeigen ist. Wie in Lemma 3.4 in [49] gezeigt wurde, ist es ausreichend, die Aussage bzgl. einer lokalen Parametrisierung zu zeigen. Hier soll der zweidimensionale Fall skizziert werden (der dreidimensionale Fall wurde in Lemma 3.8 in [49] betrachtet; dort wurde zwar nur $C^{\infty}$-Differenzierbarkeit nachgewiesen, Analytizität kann aber genauso gezeigt werden): Sei $z$ eine Parametrisierung von $\Gamma$, dann ist $I_{\theta}(z)$ eine Parametrisierung von $\Gamma_{\theta}$ und somit gilt für den normierten Tangentialvektor

$$
\tau[\theta] \circ I_{\theta} \circ z=\nabla I_{\theta}(z) \cdot z^{\prime} /\left|\nabla I_{\theta}(z) \cdot z^{\prime}\right|
$$

Es ist $|\cdot|=\sqrt{(\cdot)^{2}}$ und aus der Existenz von holomorphen Wurzelfunktionen folgt die Analytizität von $x \rightarrow|x|$ außerhalb des Nullpunkts. Somit ist die Analytizität von $\tau[\theta] \circ I_{\theta}$ und damit auch von $\nu[\theta] \circ I_{\theta}$ ersichtlich.

Nun wollen wir die Analytizität von $\tilde{u}$ nachweisen. Da der Beweis für alle Randwertprobleme sehr ähnlich ist, betrachten wir exemplarisch nur das Dirichlet-Problem und gehen am Ende dieses Abschnitts auf die Unterschiede beim Neumann-Problem ein. Die anderen Randwertprobleme (d.h. Robin- und Transmissionsproblem) können ähnlich behandelt werden.

Satz 6.6. $\theta \rightarrow \tilde{u}[\theta]$ ist analytisch von $W$ nach $\hat{C}^{m, \alpha}(\bar{\Omega})$.

Beweis. Betrachte den Operator $F: W \times \hat{C}^{m, \alpha}(\bar{\Omega}) \rightarrow C_{0}^{m-2, \alpha}(\bar{\Omega}) \times C^{m, \alpha}(\partial \Omega)$ mit

$$
F[\theta, v]=\left(\mathcal{L}[\theta, v], \mathcal{R}_{D}\left[\theta, v+u_{i} \circ I_{\theta}\right]\right)
$$

Wir interpretieren hierbei die auftretenden Funktionenräume als reelle Banachräume. Zunächst ist zu zeigen, daß $F$ analytisch ist. Für die Operatoren $\mathcal{L}$ und $\mathcal{R}_{D}$ wurde das in Lemma 6.5 gezeigt. Die Analytizität der Abbildung $\theta \rightarrow u_{i} \circ I_{\theta}$ zeigt man wie im Beweis von Lemma 6.8. Für die Anwendung des impliziten Funktionentheorems ist nun die Invertierbarkeit von $G:=F_{v}[0, u[0]] \in L\left(\hat{C}^{m, \alpha}(\bar{\Omega}), C_{0}^{m-2, \alpha}(\bar{\Omega}) \times C^{m, \alpha}(\partial \Omega)\right)$ mit

$$
G w=\left(\mathcal{L}[0, w], \mathcal{R}_{D}[0, w]\right)=\left(\Delta w+\kappa^{2} w, w\right)
$$


entscheidend. Hier wird ausgenutzt, daß $F$ bzgl. des zweiten Arguments affin ist. In Satz 4.2 wurde nun gerade die Gleichung $G=\mathcal{D}_{D}^{-1}$, d.h. die Bijektivität von $G$ nachgewiesen. Weiterhin gilt:

$$
\begin{aligned}
F[\theta, \tilde{u}[\theta]] & =\left(\left(\Delta_{\theta}+\kappa^{2}\right) \tilde{u}[\theta],\left(u\left[\left.\theta\right|_{\partial \Omega}\right]+u_{i}\right) \circ I_{\theta}\right) \\
& =\left(\left(\Delta u\left[\left.\theta\right|_{\partial \Omega}\right]+\kappa^{2} u\left[\left.\theta\right|_{\partial \Omega}\right]\right) \circ I_{\theta}, 0\right)=(0,0) .
\end{aligned}
$$

Zudem gilt $\tilde{u}[\theta]=u\left[\left.\theta\right|_{\partial \Omega}\right]$ außerhalb von $B_{R}$ bei unbeschränktem $\Omega$ und somit ist $\tilde{u}[\theta] \in \hat{C}^{m, \alpha}(\bar{\Omega})$ (die Eigenschaft $\tilde{u}[\theta] \in C^{m, \alpha}(\bar{\Omega})$ folgt aus Lemma 2.3). Daher ist der Satz über implizite Funktionen anwendbar (vgl. [9], Theorem 15.3) und nach einer eventuellen Verkleinerung von $W$ folgt die Behauptung.

Auf echten Teilmengen von $\Omega$ folgt aus obigem Satz bereits die Analytizität von $\vartheta \rightarrow u[\vartheta]:$

Satz 6.7. Sei $\Omega^{\prime} \Subset \Omega$ und $l \in \mathbb{N}$, dann existiert ein $n \in \mathbb{N}$, so daß $u: V_{n} \rightarrow C^{l}\left(\overline{\Omega^{\prime}}\right)$ analytisch ist. Weiterhin gilt

$$
\left(\Delta+\kappa^{2}\right) u^{(j)}\left[0 ; h^{j}\right]=0, \quad j \in \mathbb{N}, \quad h \in C^{m, \alpha}\left(\partial \Omega, \mathbb{R}^{d}\right)
$$

und für unbeschränktes $\Omega$ ist zudem $u_{\infty}: V_{1} \rightarrow L^{2}\left(S^{d-1}\right)$ analytisch mit

$$
u_{\infty}^{(j)}\left[0 ; h^{j}\right]=\left(\left.u^{(j)}\left[0 ; h^{j}\right]\right|_{B_{R, e}}\right)_{\infty}, \quad j \in \mathbb{N} .
$$

Beweis. Wähle ein $n \in \mathbb{N}$ mit $1 / n<\operatorname{dist}\left(\Omega^{\prime}, \Omega\right)$, dann gilt wegen $\operatorname{dist}\left(\operatorname{supp}\left(E_{n, \partial \Omega} \vartheta\right), \partial \Omega\right) \leq 1 / n$ die Gleichung $E_{n, \partial \Omega} \vartheta(x)=0$ für alle $x \in \Omega^{\prime}$ und $\vartheta \in C^{m, \alpha}\left(\partial \Omega, \mathbb{R}^{d}\right)$ und somit

$$
\Omega^{\prime}=I_{E_{n, \partial \Omega} \vartheta}\left(\Omega^{\prime}\right) \subset I_{E_{n, \partial \Omega} \vartheta}(\Omega)=\Omega_{\vartheta},
$$

d.h. $\left.u[\vartheta]\right|_{\Omega^{\prime}}$ ist wohldefiniert und es gilt

$$
\left.u[\vartheta]\right|_{\Omega^{\prime}}=\left.\tilde{u}\left[E_{n, \partial \Omega} \vartheta\right]\right|_{\Omega^{\prime}}, \quad \vartheta \in V_{n}
$$

und daher die Analytizität von $u: V_{n} \rightarrow C^{m, \alpha}\left(\Omega^{\prime}\right)$. Aus dem Darstellungssatz

$$
u[\vartheta]=\mathcal{K}_{\partial \Omega^{\prime}} u[\vartheta]-\mathcal{S}_{\partial \Omega^{\prime}} \frac{\partial u[\vartheta]}{\partial \nu}
$$

und Satz 3.1 ergibt sich die Analytizität von $u: V_{n} \rightarrow C^{l}\left(\overline{\Omega^{\prime}}\right)$. Ebenfalls aus dem Darstellungssatz folgt die Gleichung

$$
u_{\infty}[\vartheta]=c_{d} \int_{\partial B_{R}}\left[\frac{\partial e^{-i k(\cdot) y}}{\partial \nu(y)} u[\vartheta](y)-e^{-i k(\cdot) y} \frac{\partial u[\vartheta]}{\partial \nu}(y)\right] \operatorname{ds}(y)
$$

(vgl. [7], Theorem 2.5 und Gleichung (3.64); $c_{d}$ ist eine von der Dimension $d$ abhängige Konstante). Insgesamt erhalten wir somit die Gleichungen (6.1) und (6.2). 
Nun zeigen wir $u^{(j)}\left[0 ; h^{j}\right] \in C^{m-j, \alpha}(\bar{\Omega})$ für $j=0, \ldots, m$ und $h \in C^{m, \alpha}\left(\partial \Omega, \mathbb{R}^{d}\right)$. Zunächst definiert man

$$
\tilde{U}[\theta]:=E_{\Omega} \tilde{u}[\theta], \quad \theta \in W,
$$

wobei aus der Linearität und Stetigkeit von $E_{\Omega}$ sofort die Analytizität von $\tilde{U}: W \rightarrow$ $C^{m, \alpha}\left(\mathbb{R}^{d}\right)$ folgt. Weiterhin betrachten wir die Abbildung

$$
\check{U}[\theta]:=\tilde{U}[\theta] \circ I_{\theta}^{-1} .
$$

Für diese Abbildung gilt $\left.\check{U}[\theta]\right|_{\Omega_{\theta}}=u\left[\left.\theta\right|_{\partial \Omega}\right]$, denn sei $x \in \Omega_{\theta}$ und $y:=I_{\theta}^{-1}(x) \in \Omega$, dann folgt

$$
\check{U}[\theta](x)=\tilde{U}[\theta](y)=\tilde{u}[\theta](y)=u\left[\left.\theta\right|_{\partial \Omega}\right](x),
$$

d.h. $\check{U}$ stellt eine Fortsetzung von $u$ auf den ganzen $\mathbb{R}^{d}$ dar. Um Differenzierbarkeitseigenschaften von $\check{U}$ darstellen zu können, führen wir weiterhin die Operatoren $T$ und $\check{T}: W \rightarrow L\left(C\left(\mathbb{R}^{d}\right), C\left(\mathbb{R}^{d}\right)\right)$ mit

$$
T[\theta] f=f \circ I_{\theta} \text { und } \check{T}[\theta] f=f \circ I_{\theta}^{-1}
$$

ein.

Lemma 6.8. Sei $0<\alpha^{\prime}<\alpha$. Die Abbildungen $T, \check{T}: W \rightarrow L\left(C^{m, \alpha}\left(\mathbb{R}^{d}\right), C^{m-j, \alpha^{\prime}}\left(\mathbb{R}^{d}\right)\right)$ sind $j$-mal Fréchet-differenzierbar. Hierbei gilt für $f \in C^{m, \alpha}\left(\mathbb{R}^{d}\right)$ die Regularität

$$
T^{(j)}\left[\theta ; h^{j}\right] f \in C^{m-j, \alpha}\left(\mathbb{R}^{d}\right) \text { und ebenso } \check{T}^{(j)}\left[\theta ; h^{j}\right] f \in C^{m-j, \alpha}\left(\mathbb{R}^{d}\right) .
$$

Weiterhin gilt

$$
T^{(j)}\left[\theta ; h^{j}\right] f=\left(\nabla^{j} f \circ I_{\theta}\right) \cdot h^{j}, \quad j=0, \ldots, m .
$$

Beweis. Zunächst zur Abbildung $T$. Sei $x \in \mathbb{R}^{d}, f \in C^{m, \alpha}\left(\mathbb{R}^{d}\right), \theta \in W$ und $h \in$ $C^{m, \alpha}\left(\partial \Omega, \mathbb{R}^{d}\right)$ hinreichend klein, dann ist $g:[0,1] \rightarrow \mathbb{C}$ mit $g(s)=f\left(I_{\theta}(x)+\operatorname{sh}(x)\right)$ $m$-mal differenzierbar mit

$$
g^{(l)}(s)=\left(\left(\nabla^{l} f\right) \circ I_{\theta+s h}\right)(x) \cdot h^{l}(x)
$$

und aus der Taylorschen Formel (vgl. [12], Seite 174, Satz 1) folgt:

$$
g(1)=\sum_{l=0}^{j-1} \frac{1}{l !} g^{(l)}(0)+\int_{0}^{1} \frac{(1-s)^{j-1}}{(j-1) !} g^{(j)}(s) \mathrm{d} s
$$

und somit gilt:

$$
\begin{aligned}
& \left\|T[\theta+h]-\sum_{l=0}^{j} \frac{1}{l !} T^{(l)}\left[\theta ; h^{l}\right]\right\|_{m, \alpha \rightarrow m-j, \alpha^{\prime}} \\
& =\sup _{\|f\|_{m, \alpha}=1}\left\|f \circ I_{\theta+h}-\sum_{l=0}^{j} \frac{1}{l !}\left(\left(\nabla^{l} f\right) \circ I_{\theta}\right) \cdot h^{l}\right\|_{m-j, \alpha^{\prime}} \\
& =\sup _{\|f\|_{m, \alpha}=1}\left\|\int_{0}^{1} \frac{(1-s)^{j-1}}{(j-1) !}\left(\left(\nabla^{j} f\right) \circ I_{\theta+s h}-\nabla^{j} f \circ I_{\theta}\right) \cdot h^{j} \mathrm{ds}\right\|_{m-j, \alpha^{\prime}} .
\end{aligned}
$$


In der letzten Zeile haben wir die Gleichung $\int_{0}^{1} \frac{(1-s)^{j-1}}{(j-1) !} \mathrm{ds}=1 / j$ ! benutzt. Nun zeigen wir

$$
\left\|\left(\nabla^{j} f\right) \circ I_{\theta+s h}-\left(\nabla^{j} f\right) \circ I_{\theta}\right\|_{m-j, \alpha^{\prime}}=\mathrm{o}(1), \quad\|h\|_{m, \alpha} \rightarrow 0
$$

wobei die Funktion o unabhängig von $s$ ist. Der Beweis erfolgt durch Induktion. Sei zunächst $j=m, x, y \in \mathbb{R}^{d}$ und $g_{s}:=\left(\nabla^{m} f\right) \circ I_{\theta+s h}$, dann gilt für $\|f\|_{m, \alpha}=1$ :

$$
\begin{aligned}
& \left|\left(g_{s}(x)-g_{0}(x)\right)-\left(g_{s}(y)-g_{0}(y)\right)\right| \\
& =\left|\left(g_{s}(x)-g_{0}(x)\right)-\left(g_{s}(y)-g_{0}(y)\right)\right|^{\alpha^{\prime} / \alpha}\left|\left(g_{s}(x)-g_{0}(x)\right)-\left(g_{s}(y)-g_{0}(y)\right)\right|^{1-\alpha^{\prime} / \alpha} \\
& \leq\left(\left|g_{s}\right|_{\alpha}+\left|g_{0}\right|_{\alpha}\right)^{\alpha^{\prime} / \alpha}|x-y|^{\alpha^{\prime}}\left(\left|\nabla^{m} f\right|_{\alpha}\left(|h(x)|^{\alpha}+|h(y)|^{\alpha}\right)\right)^{1-\alpha^{\prime} / \alpha} \\
& \leq C|x-y|^{\alpha^{\prime}}\|h\|_{m, \alpha}^{\alpha-\alpha^{\prime}}
\end{aligned}
$$

Somit ist die Aussage für $j=m$ klar. Im Induktionsschritt setzen wir die Gültigkeit der Behauptung für ein $j \geq 1$ voraus und folgern die Aussage für $j-1$ :

$$
\begin{aligned}
\| & \left(\nabla^{j-1} f\right) \circ I_{\theta+s h}-\left(\nabla^{j-1} f\right) \circ I_{\theta} \|_{m-j+1, \alpha^{\prime}} \\
\leq & \left\|\left(\nabla^{j-1} f\right) \circ I_{\theta+s h}-\left(\nabla^{j-1} f\right) \circ I_{\theta}\right\|_{\infty} \\
& +\left\|\left(\nabla^{j} f\right) \circ I_{\theta+s h} \cdot \nabla I_{\theta+s h}-\left(\nabla^{j} f\right) \circ I_{\theta} \cdot \nabla I_{\theta}\right\|_{m-j, \alpha^{\prime}} \\
\leq & C\|h\|_{\infty}+\left\|\left(\nabla^{j} f \circ I_{\theta+s h}-\nabla^{j} f \circ I_{\theta}\right) \cdot \nabla I_{\theta+s h}\right\|_{m-j, \alpha^{\prime}} \\
& +\left\|\nabla^{j} f \circ I_{\theta}\left(\nabla I_{\theta+s h}-\nabla I_{\theta}\right)\right\|_{m-j, \alpha^{\prime}} \\
= & \mathrm{o}(1), \quad\|h\|_{m, \alpha} \rightarrow 0 .
\end{aligned}
$$

Somit gilt

$$
\left\|T[\theta+h]-\sum_{l=0}^{j-1} \frac{1}{l !} T^{(l)}\left[\theta ; h^{l}\right]\right\|_{m, \alpha \rightarrow m-j, \alpha^{\prime}}=\mathrm{o}\left(\|h\|_{m, \alpha}^{j}\right)
$$

und dies beweist die Aussage (vgl. [2], Seite 74, Korollar 2.1.29) für T.

Weiterhin gilt

$$
\check{T}[\theta] T[\theta] f=f \text { und } T[\theta] \check{T}[\theta] f=f .
$$

Die Differenzierbarkeit von $\check{T}$ kann hieraus mit einem Standardargument bewiesen werden (vgl. z.B. [57], Satz 4.2).

Betrachtet man nun die Bilinearform

$$
<\cdot, \cdot>: L\left(C^{m, \alpha}\left(\mathbb{R}^{d}\right), C^{m-j, \alpha^{\prime}}\left(\mathbb{R}^{d}\right)\right) \times C^{m, \alpha}\left(\mathbb{R}^{d}\right) \rightarrow C^{m-j, \alpha^{\prime}}\left(\mathbb{R}^{d}\right) \text { mit }<A, f>=A f,
$$

so gilt

$$
\check{U}[\theta]=<\check{T}[\theta], \tilde{U}[\theta]>
$$

und somit folgt aus der Produktregel (vgl. z.B. [63], Proposition 4.11), daß $\check{U}: W \rightarrow$ $C^{m-j, \alpha^{\prime}}\left(\mathbb{R}^{d}\right) j$-mal Fréchet-differenzierbar ist. Aus der Gleichung $\tilde{U}[\theta]=<T[\theta], \check{U}[\theta]>$ und der Produktregel gewinnen wir zudem die Darstellung

$$
\check{U}^{(j)}\left[0 ; h^{j}\right]=\tilde{U}^{(j)}\left[0 ; h^{j}\right]-\sum_{l=1}^{j}\left(\begin{array}{l}
j \\
l
\end{array}\right) \nabla^{l} \check{U}^{(j-l)}\left[0 ; h^{j-l}\right] \cdot h^{l} .
$$


Dies entspricht gerade der Leibnizschen Regel. Sei nun

$$
U_{n}[\vartheta]:=\check{U}\left[E_{n, \partial \Omega} \vartheta\right] .
$$

Satz 6.9. $U_{n}: V_{n} \rightarrow C^{m-j, \alpha^{\prime}}\left(\mathbb{R}^{d}\right)$ ist $j$-mal Fréchet-differenzierbar $(j=0, \ldots, m)$, wobei $\left.U_{n}^{(j)}\left[0 ; h^{j}\right]\right|_{\Omega}$ nicht von $n$ abhängt, d.h. für alle $h \in C^{m, \alpha}\left(\partial \Omega, \mathbb{R}^{d}\right)$ und $n_{1}, n_{2} \in \mathbb{N}$ gilt $\left.U_{n_{1}}^{(j)}\left[0 ; h^{j}\right]\right|_{\Omega}=\left.U_{n_{2}}^{(j)}\left[0 ; h^{j}\right]\right|_{\Omega}=: U^{(j)}\left[0 ; h^{j}\right]$. Für alle $\Omega^{\prime} \Subset \Omega$ gilt $\left.U^{(j)}\left[0 ; h^{j}\right]\right|_{\Omega^{\prime}}=$ $\left.u^{(j)}\left[0 ; h^{j}\right]\right|_{\Omega^{\prime}}$. Weiterhin löst $U^{(j)}\left[0 ; h^{j}\right]$ in $\Omega$ die Helmholtzgleichung, hat die DirichletRandwerte

$$
\mathcal{R}_{D} U^{(j)}\left[0 ; h^{j}\right]=-\mathcal{R}_{D}\left(\nabla^{j}\left(u_{i}+u[0]\right) \cdot h^{j}\right)-\sum_{l=1}^{j}\left(\begin{array}{l}
j \\
l
\end{array}\right) \mathcal{R}_{D}\left(\nabla^{l} U^{(j-l)}\left[0 ; h^{j-l}\right] \cdot h^{l}\right)
$$

und für unbeschränktes $\Omega$ gilt

$$
u_{\infty}^{(j)}\left[0 ; h^{j}\right]=\left(U^{(j)}\left[0 ; h^{j}\right]\right)_{\infty} .
$$

Beweis. Die Fréchet-Differenzierbarkeit folgt sofort aus der Differenzierbarkeit von $\check{U}$ und der Linearität von $E_{n, \partial \Omega}$.

Sei $h \in C^{m, \alpha}\left(\partial \Omega, \mathbb{R}^{d}\right), n_{1}, n_{2} \in \mathbb{N}$ und $x \in \Omega$. Man wähle nun $\delta_{0}>0$ mit $\delta_{0} h \in V_{n_{j}}$ $(j=1,2)$ und $y_{j}:=\left(I+E_{n_{j}, \partial \Omega} \delta_{0} h\right)^{-1}(x) \subset \Omega$, dann gilt:

$$
U_{n_{j}}\left[\delta_{0} h\right](x)=\tilde{U}\left[E_{n_{j}, \partial \Omega} \delta_{0} h\right]\left(y_{j}\right)=\tilde{u}\left[E_{n_{j}, \partial \Omega} \delta_{0} h\right]\left(y_{j}\right)=u\left[\delta_{0} h\right](x) .
$$

Somit folgt weiterhin für $0<\delta \leq \delta_{0}$ :

$$
\begin{aligned}
U_{n_{1}}^{\prime}[0 ; h](x)-U_{n_{2}}^{\prime}[0 ; h](x)=\frac{1}{\delta}\left(U_{n_{1}}^{\prime}[0, \delta h](x)-U_{n_{2}}^{\prime}[0, \delta h]\right)(x) \\
=\frac{1}{\delta}\left(\left(U_{n_{2}}[\delta h](x)-U_{n_{2}}[0](x)-U_{n_{2}}^{\prime}[0, \delta h](x)\right)\right. \\
\left.\quad-\left(U_{n_{1}}[\delta h](x)-U_{n_{1}}[0](x)-U_{n_{1}}^{\prime}[0, \delta h](x)\right)\right) \\
=\mathrm{o}(\delta) \text { für } \delta \rightarrow 0
\end{aligned}
$$

und daher $\left.U_{n_{1}}^{\prime}[0 ; h]\right|_{\bar{\Omega}}=\left.U_{n_{2}}^{\prime}[0 ; h]\right|_{\bar{\Omega}}$. Ebenso kann auch für höhere Ableitungen argumentiert werden.

Sei $\Omega^{\prime} \Subset \Omega$ und $\vartheta \in C^{m, \alpha}\left(\partial \Omega, \mathbb{R}^{d}\right)$ hinreichend klein, dann gilt $\left.U_{n}[\vartheta]\right|_{\Omega^{\prime}}=\left.u[\vartheta]\right|_{\Omega^{\prime}}$, d.h. auch $U^{(j)}[0]=u^{(j)}[0]$ und aus Satz 6.7 folgen die Aussagen bzgl. Helmholtzgleichung und Ausstrahlungsbedingung. Die Randwerte folgen aus der Darstellung (6.3) und $\left.\tilde{u}[\theta]\right|_{\partial \Omega}=-\left.\left(u_{i} \circ I_{\theta}\right)\right|_{\partial \Omega}$.

Bemerkung 6.10. Sei $u[\vartheta]$ die Lösung des Neumann-Problems in $\Omega_{\vartheta}$. Es gelten die gleichen Aussagen, wie beim Dirichlet-Problem mit einem Unterschied: Da die Neumann-Randwerte von $u[\vartheta]$ in $C^{m-1, \alpha}\left(\partial \Omega_{\vartheta}\right)$ liegen, können die Neumann-Randwerte von $U^{(j)}\left[0 ; h^{j}\right]$ im klassischen Sinn nur für $j=0, \ldots, m-1$ und nicht für $j=0, \ldots, m$ 
betrachtet werden. Zur expliziten Berechnung der Randwerte geht man folgendermaßen vor: In $\Omega_{\vartheta}$ gilt $U_{1}[\vartheta]=u[\vartheta]$ und somit

$$
\frac{\partial U_{1}[\vartheta]}{\partial \nu[\vartheta]}=\frac{\partial u[\vartheta]}{\partial \nu[\vartheta]}=-\frac{\partial u_{i}}{\partial \nu[\vartheta]}
$$

Somit kann die Gleichung

$$
\left.\left(\nabla u_{g}[\vartheta] \circ I_{\vartheta}\right) \cdot \tilde{\nu}[\vartheta]\right|_{\partial \Omega}=0 \text { mit } u_{g}[\vartheta]:=U_{1}[\vartheta]+u_{i} \text { und } \tilde{\nu}[\vartheta]:=\nu[\vartheta] \circ I_{\vartheta}
$$

differenziert werden. Sei hierzu $w[\vartheta]:=\nabla u_{g}[\vartheta] \circ I_{\vartheta}$, dann folgt aus der Leibnizschen Regel:

$$
\begin{aligned}
\left.\frac{\partial^{j}}{\partial \vartheta^{j}} w[\vartheta] \cdot \tilde{\nu}[\vartheta]\right|_{\vartheta=0} & =\sum_{l=0}^{j}\left(\begin{array}{l}
j \\
l
\end{array}\right) \nabla^{l} w^{(l)}\left[0 ; h^{l}\right] \cdot \tilde{\nu}^{(j-l)}\left[0 ; h^{j-l}\right] \\
& =\sum_{l=0}^{j}\left(\begin{array}{l}
j \\
l
\end{array}\right) \sum_{p=0}^{l}\left(\begin{array}{l}
l \\
p
\end{array}\right) \nabla^{p+1} u_{g}^{(l-p)}\left[0 ; h^{l-p}\right] \cdot h^{p} \tilde{\nu}^{(j-l)}\left[0 ; h^{j-l}\right]
\end{aligned}
$$

Beachtet man nun, daß der Summand für $l=j$ und $p=0$ gerade durch

$$
\nabla u_{g}^{j}\left[0 ; h^{j}\right] \cdot \nu=\nabla U^{(j)}\left[0 ; h^{j}\right] \cdot \nu=\mathcal{R}_{N} U^{(j)}\left[0 ; h^{j}\right]
$$

gegeben ist, so folgt insgesamt:

$$
R_{N} U^{(j)}\left[0 ; h^{j}\right]=-\sum_{\substack{l=0, \ldots, j ; p=0, \ldots .,(l, p) \neq(j, 0)}}\left(\begin{array}{l}
j \\
l
\end{array}\right)\left(\begin{array}{l}
l \\
p
\end{array}\right) \nabla^{p+1} u_{g}^{(l-p)}\left[0 ; h^{l-p}\right] \cdot h^{p} \tilde{\nu}^{(j-l)}\left[0 ; h^{j-l}\right] .
$$

\subsection{Ableitung nach der Wellenzahl}

Als nächstes wird gezeigt, daß die Lösungen der Streuprobleme analytisch von der Wellenzahl abhängen. Wir wollen die gleiche Beweisidee wie bei der Ableitung nach dem Rand verwenden. Hier ist jedoch ein Problem für unbeschränkte Gebiete $\Omega$ zu beachten: Sei $u[\kappa]$ exemplarisch die Lösung des Dirichlet-Problems für eine beliebige Wellenzahl $k$, dann gilt i.A. $u[\kappa] \notin \hat{C}^{m, \alpha}(\bar{\Omega})$, da der Raum

$$
\hat{C}^{m, \alpha}(\bar{\Omega}):=\left\{u \in C^{m, \alpha}(\bar{\Omega}):\left.\left(\Delta+\kappa^{2}\right) u\right|_{B_{R, e}}=0, \quad u \text { erfüllt die SAB }\right\}
$$

von einer bestimmten Wellenzahl abhängt. Dieser Problem umgehen wir, in dem wir entweder nur beschränkte Gebiete betrachten, oder im Fall des Transmissionsproblems die Wellenzahl im Außenraum fixieren und nur die Wellenzahlen in den inneren Gebieten variieren. Im folgenden werden wir nur das Dirichlet-Problem bei einem beschränkten Gebiet $\Omega$ betrachten. Die anderen Probleme können dann völlig analog behandelt werden. Sei $W \subset \mathbb{C}$ eine offene Teilmenge von Wellenzahlen, die keine

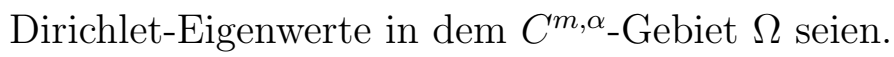


Satz 6.11. Die Abbildung $\kappa \rightarrow u[\kappa]\left(W \rightarrow C^{m, \alpha}(\bar{\Omega})\right)$ ist analytisch und es gilt

$$
\left(\Delta+\kappa^{2}\right) u^{(j)}[\kappa]=-2 j \kappa u^{(j-1)}[\kappa]-j(j-1) u^{(j-2)}[\kappa]
$$

für $j \in \mathbb{N}$ und $u^{(-1)}[\kappa]:=0$. Als Randbedingung ergibt sich

$$
\mathcal{R}_{D} u^{(j)}[\kappa]=-\mathcal{R}_{D} \frac{\partial^{j}}{\partial \kappa^{j}} u_{i}
$$

Beweis. Betrachte den Operator $F: W \times C^{m, \alpha}(\bar{\Omega}) \rightarrow C^{m-2, \alpha}(\bar{\Omega}) \times C^{m, \alpha}(\partial \Omega)$ mit

$$
F[\kappa, v]=\left(\left(\Delta+\kappa^{2}\right) v, \mathcal{R}_{D}\left[v+u_{i}\right]\right) .
$$

Zunächst ist zu zeigen, daß $F$ analytisch ist. Für die Operatoren $\Delta+\kappa^{2}$ und $\mathcal{R}_{D}$ ist das klar. Die Analytizität der Abbildung $\kappa \rightarrow u_{i}[\kappa]$ folgt aus der Analytizität von $u_{i}$. Für die Anwendung des impliziten Funktionentheorems ist die Invertierbarkeit von $G:=F_{v}[\kappa, u[\kappa]] \in L\left(C^{m, \alpha}(\bar{\Omega}), C^{m-2, \alpha}(\bar{\Omega}) \times C^{m, \alpha}(\partial \Omega)\right)$ mit

$$
G w=\left(\left(\Delta+\kappa^{2}\right) w, \mathcal{R}_{D}[w]\right)=\left(\Delta w+\kappa^{2} w, w\right)
$$

entscheidend. In Satz 4.2 wurde nun gerade die Gleichung $G=\mathcal{D}_{D}^{-1}$, d.h. die Bijektivität von $G$ nachgewiesen. Weiterhin gilt:

$$
F[\kappa, u[\kappa]]=\left(\left(\Delta+\kappa^{2}\right) u[\kappa], u[\kappa]+u_{i}\right)=(0,0) .
$$

Daher ist der Satz über implizite Funktionen anwendbar (vgl. [9], Theorem 15.3) und die erste Behauptung folgt.

Die Aussage bzgl. der Helmholtzgleichung wird durch Induktion aus der Gleichung $\left(\Delta+\kappa^{2}\right) u[\kappa]=0$ gezeigt und die Randbedingung ergibt sich durch Differenzieren der Gleichung $\mathcal{R}_{D}\left(u[\kappa]+u_{i}\right)=0$.

Im Gegensatz zur Ableitung nach dem Rand ist bei der Ableitung nach der Wellenzahl also eine inhomogene Helmholtzgleichung zu lösen. Dies ist aus numerische Sicht unbefriedigend, da der Aufwand hierfür erheblich höher ist, als bei der Lösung eines Randwertproblems zur homogenen Helmholtzgleichung. Stattdessen soll ein anderes Verfahren verwendet wurden, das für die Ableitung nach dem Rand bereits in der Arbeit [49] beschrieben wurde. Hier betrachten wir exemplarisch den Potentialansatz beim Transmissionsproblem. Das Fernfeld des gestreuten Feldes ist von der Form $u_{\infty}=F_{\infty} M^{-1} I_{\rho} f$ mit dem Fernfeldoperator $F_{\infty}$, einem matrixwertigen Integraloperator $M$, in den die Randoperatoren eingehen und den Randwerten $I_{\rho} f$, wobei nur der Operator $M$ von den inneren Wellenzahlen abhängt. Um nun das Fernfeld der Ableitungen nach der Wellenzahl zu bestimmen, kann diese Formel nach der Kettenregel differenziert werden, d.h. es gilt

$$
u_{\infty}^{\prime}[\kappa]=-F_{\infty} M^{-1}[\kappa] M^{\prime}[\kappa] M^{-1}[\kappa] G .
$$

$\mathrm{Zu}$ zeigen ist also, daß die Abbildung $\kappa \rightarrow M[\kappa]$ differenzierbar ist, wobei $M[\kappa]$ Kombinationen von Integraloperatoren aus Satz 3.2 enthält. Hierbei betrachten wir diese Operatoren jetzt als Abbildungen von einer Teilmenge der komplexen Zahlen in einen Raum von linearen Operatoren. Hierfür gehen wir wie in Satz 3.5. aus [49] vor. 
Satz 6.12. Die Operatoren $S, K, K^{*} \in \mathbb{C} \rightarrow L(C(\partial \Omega), C(\partial \Omega))$ und der Operator $T \in \mathbb{C} \rightarrow L\left(C^{1, \alpha}(\partial \Omega), C(\partial \Omega)\right)$ sind differenzierbar, wobei die Ableitungen durch differenzieren der jeweiligen Kerne entstehen.

Beweis. Betrachtet wird nur der zweidimensionale Fall, die Situation im $\mathbb{R}^{3}$ ist analog beschreibbar. Zur Abkürzung wird im folgenden $H_{0}$ statt $H_{0}(\kappa|x-y|)$ und $H_{1}$ statt $H_{1}(\kappa|x-y|)$ geschrieben. Zunächst zum Einfachschichtpotential:

$$
\begin{aligned}
\frac{\partial}{\partial \kappa} H_{0} & =-H_{1}|x-y|, \\
\frac{\partial^{2}}{\partial \kappa^{2}} H_{0} & =\frac{1}{\kappa} H_{1}|x-y|-H_{0}|x-y|^{2} .
\end{aligned}
$$

Für den Kern des Operators $K$ gilt:

$$
\begin{aligned}
\frac{\partial}{\partial \kappa}\left(\kappa H_{1} \frac{(x-y) \cdot \nu(y)}{|x-y|}\right) & =\kappa H_{0}(x-y) \cdot \nu(y) \\
\frac{\partial^{2}}{\partial \kappa^{2}}\left(\kappa H_{1} \frac{(x-y) \cdot \nu(y)}{|x-y|}\right) & =\left(H_{0}-H_{1}|x-y|\right)(x-y) \cdot \nu(y) .
\end{aligned}
$$

Durch Vertauschen von $\nu(y)$ mit $\nu(x)$ ist auch der Kern von $K^{*}$ abgehandelt. Nun noch zum Operator $T$ :

$$
\begin{aligned}
& \frac{\partial}{\partial \kappa}\left(\left(\kappa^{2} H_{0}-\frac{\kappa}{|x-y|} H_{1}\right) \frac{(x-y) \cdot \nu(y)(x-y) \cdot \nu(x)}{|x-y|^{2}}+\kappa H_{1} \frac{\nu(y) \cdot \nu(x)}{|x-y|}\right) \\
& =\left(\kappa H_{0}-\kappa^{2} H_{1}|x-y|\right) \frac{(x-y) \cdot \nu(y)(x-y) \cdot \nu(x)}{|x-y|^{2}}+\kappa H_{0} \nu(y) \cdot \nu(x), \\
& \frac{\partial^{2}}{\partial \kappa^{2}}\left(\left(\kappa^{2} H_{0}-\frac{\kappa}{|x-y|} H_{1}\right) \frac{(x-y) \cdot \nu(y)(x-y) \cdot \nu(x)}{|x-y|^{2}}+\kappa H_{1} \frac{\nu(y) \cdot \nu(x)}{|x-y|}\right) \\
& =\left(H_{0}\left(1-\kappa^{2}|x-y|^{2}\right)-2 \kappa H_{1}|x-y|\right) \frac{(x-y) \cdot \nu(y)(x-y) \cdot \nu(x)}{|x-y|^{2}} \\
& \quad+\left(H_{0}-\kappa H_{1}|x-y|\right) \nu(y) \cdot \nu(x) .
\end{aligned}
$$

Alle Kernfunktionen sind stetig fortsetzbar, so daß die Aussage nun genau wie in Satz 3.5 aus [49] bewiesen werden kann.

\subsection{Ableitung nach der Transmissionskonstanten $\rho$}

Die Analytizität der Lösung des Transmissionsproblems bzgl. des Übergangsparameters $\rho$ ist mit den verwendeten Hilfsmitteln sehr einfach nachweisbar. Sei der Wellenzahlvektor $\kappa \in \mathbb{C}^{L+1}$ gegeben und $W \subset \mathbb{C}^{L}$ eine offene Teilmenge der Parameter $\rho$, so daß das Transmissionsproblem eindeutig lösbar ist. Der Randwertoperator $\mathcal{R}_{T}: W \times \hat{C}^{m, \alpha} \rightarrow C^{m, m-1, \alpha}(\Gamma)$ sei definiert durch

$$
\mathcal{R}_{T}[\rho, w]=\left(w_{j}-w_{v(j)}, \frac{\partial}{\partial \nu}\left(w_{j}-\rho_{j} w_{v(j)}\right)\right)_{j=1}^{L} .
$$


Für die einfallende ebene Welle $u_{i}$ definieren wir Transmissionsrandwerte $U_{i} \in$ $C^{m, m-1, \alpha}(\Gamma)$ durch folgende Vorgehensweise. Für einen äußeren Rand $\Gamma_{j}$ sei $U_{i, j}:=$ $\mathcal{R}_{C, \Gamma_{j}} u_{i}$, anderenfalls gelte $U_{i, j}=0$. Für diese Randwerte betrachten wir jetzt das Problem 5.1.

Satz 6.13. Die Abbildung $\rho \rightarrow u[\rho]$ (d.h. $W \rightarrow \hat{C}^{m, \alpha}$ ) ist analytisch und es gilt $\mathcal{L}_{T} u^{(j)}[\rho]=0$ für $j \in \mathbb{N}$. Als Randbedingung ergibt sich

$$
\mathcal{R}_{T}\left[\rho, u^{(j)}[\rho]\right]=\left(0,-\frac{\partial}{\partial \nu}\left(\left(u[\rho]+U_{i}\right)_{v(j)}\right)_{j=1}^{L}\right) .
$$

Beweis. Sei $F: W \times \hat{C}^{m, \alpha} \rightarrow C_{0}^{m-2, \alpha} \times C^{m, m-1, \alpha}(\Gamma)$ definiert durch $F[\rho, v]=$ $\left(\mathcal{L}_{T} v, \mathcal{R}_{T}[\rho, v]\right)$, dann ist $F$ analytisch und $F_{v}[\rho, u[\rho]]$ ist beschränkt invertierbar. Zudem gilt $F[\rho, u[\rho]]=0$, so daß wiederum der Satz über implizite Funktionen (vgl. [9], Theorem 15.3) anwendbar ist.

Die zweite Aussage folgt aus der Linearität von $\mathcal{L}_{T}$ und die letzte Behauptung folgt durch differenzieren der Gleichung $\mathcal{R}_{T}\left[\rho, u[\rho]+U_{i}\right]=0$.

\subsection{Vergleich mit anderen Resultaten}

Am Ende dieses Kapitels sollen die Ergebnisse mit anderen verglichen werden. Es exitieren insbesondere für die Gebietsableitung eine ganze Reihe von Artikeln, die sich mit diesem Thema befassen. Die ersten Untersuchungen scheinen die Störungen von Gebieten durch Vektorfelder betrachtet zu haben (vgl. den Artikel von Simon [58] aus dem Jahr 1980 und die darin angegebene Literatur. Als Anwendungsbeispiel wurde hier die Differenzierbarkeit der Lösung einer Laplacegleichung betrachtet. Dies wurde für Lipschitz-stetige Ränder auch auf die Helmholtzgleichung übertragen (vgl. [11] und auch [18]). Allen Arbeiten ist gemeinsam, daß sie die schwache Formulierung des Randwertproblems betrachten und die Aussagen in entsprechenden Sobolev-Räumen beweisen.

Unter Benutzung des schwachen Lösungsbegriffs konnte auch Kirsch in [31] die Existenz einer Gebietsableitung für den schallweichen Fall nachweisen. Dieser Zugang wurde später auf andere Randbedingungen übertragen (vgl. [10], [21]). In der Dissertation von Hohage ([27]) werden Ideen von beiden Ansätzen verwendet und Analytizität des gestreuten Feldes im schallweichen und -harten Fall unter sehr schwachen Voraussetzungen an die Randglätte bewiesen. Auch hier wird die schwache Formulierung des Problems benutzt.

Einen anderer Zugang benutzte Potthast (vgl. [49], [48], [51]). Er bewies die Differenzierbarkeit des gestreuten Feldes durch einen Integralgleichungsansatz. Die Herleitung der Randwerte des differenzierten Feldes wurde in [57] vereinfacht.

In einer Arbeit von Bruno und Reitich (vgl. [3]) wird eine holomorphe Formulierung des Problems benutzt, um analytische Abhängigkeit nachzuweisen. Hierbei ist der Rand von der Form $\delta f(x)$ und die Differenzierbarkeit wird bzgl. des Parameters $\delta$ nachgewiesen. Diese Ergebnisse wurden in den Arbeiten [4], [54] und [55] benutzt, um 
aufbauend auf einer Taylorentwicklung des gestreuten Feldes ein numerisches Verfahren zur Lösung von Streuproblemen zu entwickeln. 


\section{Numerische Ergebnisse}

In dem letzten Kapitel wollen wir einige konkrete numerische Beispiele betrachten. Von nun an untersuchen wir nur noch den zweidimensionalen Fall. Als Randwertproblem betrachten wir das Transmissionsproblem, wobei auch mehrere Gebiete zugelassen sind. Zunächst wollen wir kurz darstellen, wie das direkte Transmissionsproblem numerisch gelöst werden kann. Für das angegebene Verfahren führen wir eine Fehleranalyse in Hölder-Räumen durch. In einem zweiten Schritt geben wir eine Möglichkeit zur Berechnung der Fréchet-Ableitungen nach dem Rand, der Wellenzahl und der Übergangskonstanten $\rho$ an. Der dritte Abschnitt beschäftigt sich dann mit inversen Transmissionsproblemen, d.h. mit der Rekonstruktion der auftretenden Parameter aus den Fernfelddaten für eine oder mehrere einfallende ebene Wellen. Am Ende geben wir eine kurze Literaturübersicht.

\subsection{Numerische Lösung des direkten Problems}

Die Grundidee für die Diskretisierung der auftretenden Integraloperatoren besteht in einer geeigneten Abspaltung der Singularitäten und exakter Integration in dem Raum der trigonometrischen Polynome. Für eine ausführlichere Darstellung der Methode verweisen wir auf Kress (vgl. [37], [36]) und die Diplomarbeiten [15] und [65], die sich mit der numerischen Lösung des direkten Transmissionsproblems beschäftigen. Wir werden hauptsächlich Integralgleichungen zweiter Art betrachten. Diese werden häufig mit dem NyströmVerfahren diskretisiert (vgl. [37] für eine Analyse von verschiedenen Verfahren zur Lösung von Integralgleichungen zweiter Art). Für die Lösung der hier betrachteten inversen Probleme ist es je-

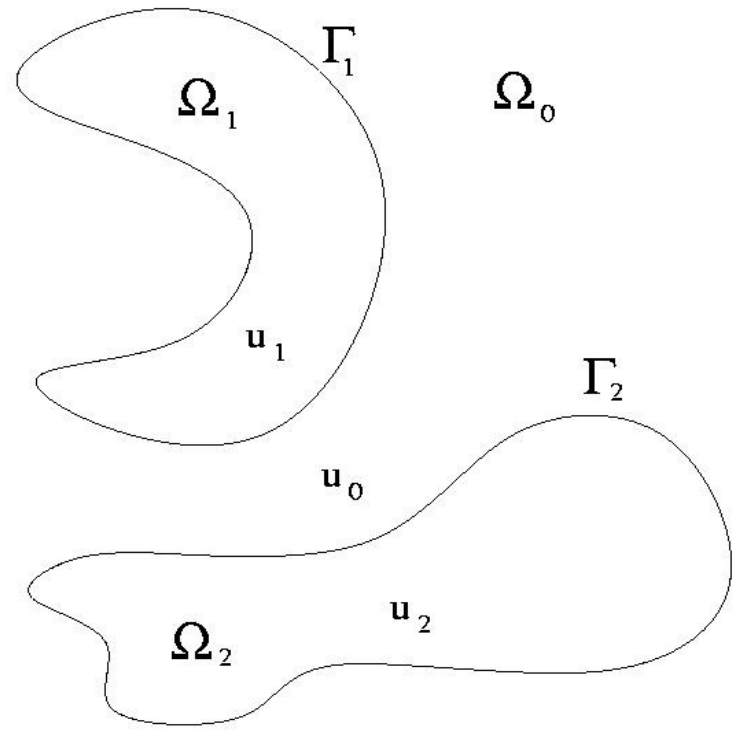

Abbildung 7.1: Beispielgeometrie doch wichtig, daß die Näherungslösungen trigonometrische Polynome sind. Daher ist es in dieser Situation am günstigsten, die betrachteten Integralgleichungen durch Projektionsverfahren mit trigonometrischen 
Polynomen zu lösen. Das volldiskrete System ist zwar das gleiche wie beim NyströmVerfahren, die Näherungslösungen sind bei der von uns gewählten Methode trigonometrische Polynome und dies ist beim Nyström-Verfahren im allgemeinen nicht der Fall.

Exemplarisch betrachten wir hier einen Potentialansatz für das Transmissionsproblem. Das Ziel besteht also in der numerischen Lösung des Gleichungssystems (5.2), das wir mit $M \varphi=I_{\rho} f$ bezeichnet hatten. Um die Darstellung etwas anschaulicher zu gestalten, wollen wir das folgende an einem konkreten Beispiel für zwei nebeneinanderliegen Gebiete betrachten (vgl. Abbildung 7.1). Wir setzen $\partial \Omega \in C^{m+2, \alpha}$ für $m \in \mathbb{N}_{\geq 2}$ voraus. Wie in Kapitel 5 beschrieben, verwenden wir die folgenden Ansatzfunktionen:

$$
\begin{aligned}
& u_{0}:=\mathcal{K}_{1, a} \varphi_{1,1}+d_{1} \mathcal{S}_{1, a} \varphi_{1,2}+\mathcal{K}_{2, a} \varphi_{2,1}+d_{2} \mathcal{S}_{2, a} \varphi_{2,2}, \\
& u_{1}:=\rho_{1} \mathcal{K}_{1, i} \varphi_{1,1}+\mathcal{S}_{1, i} \varphi_{1,2}, \\
& u_{2}:=\rho_{2} \mathcal{K}_{2, i} \varphi_{2,1}+\mathcal{S}_{2, i} \varphi_{2,2} .
\end{aligned}
$$

Zu lösen sind die Gleichungen

$$
\mathcal{R}_{T, \Gamma_{1}, \rho_{1}}\left(u_{1}, u_{0}\right)=I_{\rho_{1}} f_{1} \text { und } \mathcal{R}_{T, \Gamma_{2}, \rho_{2}}\left(u_{2}, u_{0}\right)=I_{\rho_{2}} f_{2} .
$$

Dies entspricht einem System $M \varphi=I_{\rho} f$ mit $M=M_{d}+M_{k}$. Wir verwenden nun die Aufspaltung von $M$ in der Form (5.3). Somit ist $M_{d}$ eine Diagonalmatrix und $M_{k}$ hat folgende Gestalt:

$$
M_{k}=\left(\begin{array}{cccc}
\rho_{1} K_{1, i}-K_{1, a} & S_{1, i}-d_{1} S_{1, a} & -\left.\mathcal{K}_{2, a}\right|_{\Gamma_{1}} & -\left.d_{2} \mathcal{S}_{2, a}\right|_{\Gamma_{1}} \\
\rho_{1}\left(T_{1, i}-T_{1, a}\right) & K_{1, i}^{*}-\rho_{1} d_{1} K_{1, a}^{*} & -\left.\rho_{1} \frac{\partial}{\partial \nu_{1}} \mathcal{K}_{2, a}\right|_{\Gamma_{1}} & -\left.\rho_{1} d_{2} \frac{\partial}{\partial \nu_{1}} \mathcal{S}_{2, a}\right|_{\Gamma_{1}} \\
-\left.\mathcal{K}_{1, a}\right|_{\Gamma_{2}} & -\left.d_{1} \mathcal{S}_{1, a}\right|_{\Gamma_{2}} & \rho_{2} K_{2, i}-K_{2, a} & S_{2, i}-d_{2} S_{2, a} \\
-\left.\rho_{2} \frac{\partial}{\partial \nu_{2}} \mathcal{K}_{1, a}\right|_{\Gamma_{2}} & -\left.\rho_{2} d_{1} \frac{\partial}{\partial \nu_{2}} \mathcal{S}_{1, a}\right|_{\Gamma_{2}} & \rho_{2}\left(T_{2, i}-T_{2, a}\right) & K_{2, i}^{*}-\rho_{2} d_{2} K_{2, a}^{*}
\end{array}\right) .
$$

Zur Lösung des inversen Problems (d.h. der Rekonstruktion des Randes bzw. der auftretenden Konstanten) werden wir auch die Cauchy-Daten von $u_{0}, u_{1}$ und $u_{2}$ benötigen. Ausreichend ist hierbei die Kenntnis der Cauchy-Daten von $u_{0}$. Hieraus ermittelt man die Cauchy-Daten von $u_{1}$ und $u_{2}$ durch die Transmissionsrandbedingungen. Die Cauchy-Daten $u_{c}$ von $u_{0}$ ergeben sich aus der Gleichung

$$
\begin{aligned}
u_{c}=\left(\begin{array}{c}
\left.u_{0}\right|_{\Gamma_{1}} \\
\frac{\partial u_{0}}{\partial \nu_{1}} \\
\left.u_{0}\right|_{\Gamma_{2}} \\
\frac{\partial u_{0}}{\partial \nu_{2}}
\end{array}\right)=Q_{d} \varphi+Q_{k} \varphi:=\left(\begin{array}{cccc}
1 / 2 & 0 & 0 & 0 \\
0 & -d_{1} / 2 & 0 & 0 \\
0 & 0 & 1 / 2 & 0 \\
0 & 0 & 0 & -d_{2} / 2
\end{array}\right) \varphi \\
+\left(\begin{array}{cccc}
K_{1, a} & d_{1} S_{1, a} & \left.\mathcal{K}_{2, a}\right|_{\Gamma_{1}} & \left.d_{2} \mathcal{S}_{2, a}\right|_{\Gamma_{1}} \\
T_{1, a} & d_{1} K_{1, a}^{*} & \left.\frac{\partial}{\partial \nu_{1}} \mathcal{K}_{2, a}\right|_{\Gamma_{1}} & \left.d_{2} \frac{\partial}{\partial \nu_{1}} \mathcal{S}_{2, a}\right|_{\Gamma_{1}} \\
\left.\mathcal{K}_{1, a}\right|_{\Gamma_{2}} & \left.d_{1} \mathcal{S}_{1, a}\right|_{\Gamma_{2}} & K_{2, a} & d_{2} S_{2, a} \\
\left.\frac{\partial}{\partial \nu_{2}} \mathcal{K}_{1, a}\right|_{\Gamma_{2}} & \left.d_{1} \frac{\partial}{\partial \nu_{2}} \mathcal{S}_{1, a}\right|_{\Gamma_{2}} & T_{2, a} & d_{2} K_{2, a}^{*}
\end{array}\right) \varphi
\end{aligned}
$$

Wir fixieren jetzt eine $2 \pi$-periodische Parametrisierung $z$ und betrachten von nun an alle Operatoren als Abbildungen von einem Raum $2 \pi$-periodischer Funktionen in einen 
Raum $2 \pi$-periodischer Funktionen. Wir wollen diese Operatoren aber weiterhin mit den gleichen Symbolen bezeichnen.

Der Trick besteht nun in einer geeigneten Zerlegung der Komponenten von $M_{k}$ und $Q_{k}$. Hierzu definieren wir folgende Operatoren:

$$
\begin{aligned}
B_{1}(k) \varphi(t) & :=\int_{0}^{2 \pi} k(t, \tau) \varphi(\tau) \mathrm{d} \tau \\
B_{2}(k) \varphi(t) & :=\int_{0}^{2 \pi} \ln \left(4 \sin ^{2} \frac{t-\tau}{2}\right) k(t, \tau) \varphi(\tau) \mathrm{d} \tau \\
B_{3} \varphi(t) & :=\frac{1}{2 \pi} \int_{0}^{2 \pi} \cot \frac{t-\tau}{2} \varphi^{\prime}(\tau) \mathrm{d} \tau .
\end{aligned}
$$

Auf dem Einheitskreis entspricht $B_{3}$ gerade dem Operator $T_{0}$ und $B_{2}(1 / 2 \pi)$ dem Operator $S$. Nun benutzen wir folgenden Zerlegungen:

$$
\begin{aligned}
S & =B_{1}\left(k_{S}\right)+B_{2}\left(w_{S}\right), & & \\
K & =B_{1}\left(k_{K}\right)+B_{2}\left(w_{K}\right), & K^{*} & =B_{1}\left(k_{K^{*}}\right)+B_{2}\left(w_{K^{*}}\right), \\
T_{i}-T_{a} & =B_{1}\left(k_{T_{i} T_{a}}\right)+B_{2}\left(w_{T_{i} T_{a}}\right), & T_{a} & =B_{1}\left(k_{T}\right)+B_{2}\left(w_{T}\right)+B_{3}, \\
\mathcal{S} & =B_{1}\left(k_{\mathcal{S}}\right), & \mathcal{K} & =B_{1}\left(k_{\mathcal{K}}\right), \\
\frac{\partial}{\partial \nu_{j}} \mathcal{S}_{l} & =B_{1}\left(k_{\mathcal{S}_{j l}}\right), & \frac{\partial}{\partial \nu_{j}} \mathcal{K}_{l} & =B_{1}\left(k_{\mathcal{K}_{j l}}\right), \quad j, l \in\{1,2\}, \quad j \neq l .
\end{aligned}
$$

Hier haben wir die Indices an den Operatoren weggelassen, da die Zerlegungen für alle Operatoren gleichermaßen erfolgt. Für eine ausführliche Herleitung verweisen wir auf auf Kapitel 3.5 in [7], Beispiel 12.14 in [37] und die Artikel [36] und [41].

Alle Kernfunktionen sind $2 \pi$-periodisch und ungefähr so glatt wie der Rand $\Gamma$. Exemplarisch betrachten wir den Kern $k$ des Operators $S$, um zu zeigen, wie man diese Aufspaltungen erhält:

$$
\begin{aligned}
k(t, \tau)= & \frac{i}{4} H_{0}(\kappa|z(t)-z(\tau)|)\left|z^{\prime}(\tau)\right| \\
= & \underbrace{\frac{i}{4} H_{0}(\kappa|z(t)-z(\tau)|)\left|z^{\prime}(\tau)\right|+\frac{1}{2 \pi} J_{0}(\kappa|z(t)-z(\tau)|) \ln (|z(t)-z(\tau)|)\left|z^{\prime}(\tau)\right|}_{=: p(t, \tau)} \\
& -\frac{1}{2 \pi} J_{0}(\kappa|z(t)-z(\tau)|) \ln (|z(t)-z(\tau)|)\left|z^{\prime}(\tau)\right| \\
= & p(t, \tau)-\frac{1}{4 \pi} J_{0}\left(\kappa_{0}|z(t)-z(\tau)|\right)\left|z^{\prime}(\tau)\right| \ln \frac{|z(t)-z(\tau)|^{2}}{4 \sin ^{2} \frac{t-\tau}{2}} \\
& -\frac{1}{4 \pi} J_{0}(\kappa|z(t)-z(\tau)|)\left|z^{\prime}(\tau)\right| \ln \left(4 \sin ^{2} \frac{t-\tau}{2}\right) \\
= & : k_{S}(t, \tau)+w_{S}(t, \tau) \ln \left(4 \sin ^{2} \frac{t-\tau}{2}\right) .
\end{aligned}
$$


Aus der Reihendarstellung der Hankelfunktion folgt $k_{S} \in C^{m+1, \alpha}([0,2 \pi] \times[0,2 \pi])$. Mit Hilfe der Gleichung

$$
z(t)-z(\tau)=(t-\tau) \int_{0}^{1} z^{\prime}(\tau+s(t-\tau)) \mathrm{d} s
$$

zeigt man

$$
\ln \frac{|z(t)-z(\tau)|^{2}}{\sin ^{2} \frac{t-\tau}{2}} \in C^{m+1, \alpha}([0,2 \pi] \times[0,2 \pi]) .
$$

In den Operatoren $K$ und $K^{*}$ treten Terme der Form $(z(t)-z(\tau)) \nu(\tau) /|z(t)-z(\tau)|^{2}$ bzw. $(z(t)-z(\tau)) \nu(t) /|z(t)-z(\tau)|^{2}$ auf. Hier entwickelt man $z$ bis zur zweiten Ableitung und folgert, daß die Terme in $C^{m, \alpha}([0,2 \pi] \times[0,2 \pi])$ liegen (vgl. auch hierzu Lemma 3.6 und auch [43], Seite 258f.). Mit ähnlichen Überlegungen erhalten wir folgendes Ergebnis:

$$
k_{S}, w_{S}, k_{K}, w_{K}, k_{\mathcal{S}}, k_{\mathcal{K}}, k_{K^{*}}, w_{K^{*}}, k_{T_{i} T_{a}}, w_{T_{i} T_{a}}, k_{\mathcal{S}_{j l}}, k_{\mathcal{K}_{j l}} \in C^{m, \alpha}([0,2 \pi] \times[0,2 \pi]) .
$$

Die Kerne $k_{T}$ und $w_{T}$ wurden in [36] analysiert. Sie haben folgende Regularität: $k_{T}$, $w_{T} \in C^{m-1, \alpha}([0,2 \pi] \times[0,2 \pi])$.

Nun erklären wir Approximationsoperatoren, die auf trigonometrischer Interpolation beruhen. Für $n \in \mathbb{N}, n$ gerade, verwenden wir äquidistante Knotenpunkte

$$
t_{j}^{(n)}:=\frac{2 \pi j}{n}, \quad j=0, \ldots, n-1 .
$$

Bzgl. des n-dimensionalen Raums $T_{n}$ der trigonometrischen Polynome der Form

$$
v(t)=\sum_{m=0}^{n / 2} a_{m} \cos m t+\sum_{m=1}^{n / 2-1} b_{m} \sin m t
$$

und der Knotenpunkte $t_{j}^{(n)}$ ist das Interpolationsproblem im Raum der stetigen Funktionen eindeutig lösbar. Mit $P_{n}: C[0,2 \pi] \rightarrow T_{n}$ bezeichnen wir den zugehörigen Interpolationsoperator. Nun definieren wir folgende Approximationsoperatoren:

$$
\begin{aligned}
B_{1, n}(k) \varphi(t) & :=\int_{0}^{2 \pi} P_{n}(k(t, \cdot) \varphi)(\tau) \mathrm{d} \tau \\
B_{2, n}(k) \varphi(t) & :=\int_{0}^{2 \pi} \ln \left(4 \sin ^{2} \frac{t-\tau}{2}\right) P_{n}(k(t, \cdot) \varphi)(\tau) \mathrm{d} \tau, \\
B_{3, n} \varphi(t) & :=\frac{1}{2 \pi} \int_{0}^{2 \pi} \cot \frac{t-\tau}{2}\left(P_{n} \varphi\right)^{\prime}(\tau) \mathrm{d} \tau .
\end{aligned}
$$

Ersetzt man die Operatoren in $M_{k}$ und $Q_{k}$ durch die entsprechenden Approximationsoperatoren, so erhalten wir Operatormatrizen $M_{k, n}$ und $Q_{k, n}$ und betrachten nun das Gleichungssystem

$$
\left(M_{d}+P_{n} M_{k, n}\right) \varphi_{n}=I_{\rho} P_{n} f .
$$


Mit $P_{n} M_{k, n}$ verstehen wir dabei die Anwendung des Operators $P_{n}$ auf jede Komponente von $M_{k, n}$. Falls $\varphi_{n}$ eine Lösung dieses Systems ist, so liegen die Komponenten von $\varphi_{n}$ in $T_{n}$. Dieses semidiskrete System kann nun durch explizite Quadraturformeln in ein äquivalentes endlichdimensionales System überführt werden (vgl. z.B. [7], Kapitel 3.5, und die anderen bereits zitierten Texte zu diesem Thema). Für eine Lösung $\varphi_{n}$ erhalten wir eine Näherung $u_{c, n}$ an die Cauchy-Randwerte $u_{c}$ durch

$$
u_{c, n}=\left(Q_{d}+P_{n} Q_{k, n}\right) \varphi_{n} .
$$

Wiederum verstehen wir unter $P_{n} Q_{k, n}$ die Anwendung von $P_{n}$ auf jede Komponente von $Q_{k, n}$.

Die Konvergenz- und Fehleranalyse soll nun in Hölder-Räumen durchgeführt werden. Grundlage ist hierbei der Artikel [36], in dem ein Neumann-Problem betrachtet wurde. Zunächst verschärfen wir das Lemma 4.1 aus dem eben genannten Artikel. Wir definieren hierzu für $l, p \in \mathbb{N}_{0}, l \leq p$, folgenden Hölder-Raum:

$$
\begin{aligned}
C^{p, l, \alpha}([0,2 \pi] \times[0,2 \pi]):= & \left\{\psi \in C^{l, \alpha}([0,2 \pi] \times[0,2 \pi]): \forall t_{2} \in[0,2 \pi]: \psi\left(\cdot, t_{2}\right) \in C^{p, \alpha}[0,2 \pi]\right. \\
& \text { und } \left.\frac{\partial^{p-l}}{\partial t_{1}^{p-l}} \psi \in C^{l, \alpha}([0,2 \pi] \times[0,2 \pi])\right\} .
\end{aligned}
$$

Lemma 7.1. Sei $\psi \in C^{p, p-1, \alpha}([0,2 \pi] \times[0,2 \pi])(p \in \mathbb{N})$, dann gilt für die Funktion

$$
u(t):=\int_{0}^{2 \pi} \ln \left(4 \sin ^{2} \frac{t-\tau}{2}\right) \psi(t, \tau) \mathrm{d} \tau
$$

die Abschätzung

$$
\|u\|_{p, \alpha} \leq C \max _{j=0,1}\left\|\frac{\partial^{j} \psi}{\partial t^{j}}\right\|_{p-1, \alpha},
$$

wobei die Konstante $C$ von $p$ und $\alpha$ abhängt.

Beweis. Der Nachweis erfolgt durch Induktion. Sei zunächst $p=1$. In Lemma 4.1 aus [36] wurde die Ungleichung

$$
\|u\|_{0, \alpha} \leq C \max _{j=0,1}\left\|\frac{\partial \psi}{\partial t}\right\|_{\infty}
$$

bewiesen, so daß nur noch $u^{\prime}$ zu untersuchen ist. Differenziert man $u$, ergibt sich folgende Darstellung:

$$
\begin{aligned}
u^{\prime}(t)= & \int_{0}^{2 \pi}\left(\frac{\partial}{\partial t} \ln \left(4 \sin ^{2} \frac{t-\tau}{2}\right)\right)(\psi(t, \tau)-\psi(\tau, \tau)) \mathrm{d} \tau \\
& +\int_{0}^{2 \pi}\left(\frac{\partial}{\partial t} \ln \left(4 \sin ^{2} \frac{t-\tau}{2}\right)\right) \psi(\tau, \tau) \mathrm{d} \tau \\
& +\int_{0}^{2 \pi} \ln \left(4 \sin ^{2} \frac{t-\tau}{2}\right) \frac{\partial \psi}{\partial t}(t, \tau) \mathrm{d} \tau \\
= & : I_{1}(t)+I_{2}(t)+I_{3}(t) .
\end{aligned}
$$


Da $\psi$ nach der ersten Variablen differenzierbar ist, gewinnen wir die Gleichung

$$
\psi(t, \tau)-\psi(\tau, \tau)=(t-\tau) \int_{0}^{1} \frac{\partial \psi}{\partial t}(\tau+s(t-\tau), \tau) \mathrm{d} s=:(t-\tau) \chi(t, \tau)
$$

Hierbei ist die Funktion $\chi$ Hölder-stetig, d.h. es gilt $\left\|I_{1}\right\|_{0, \alpha} \leq c\left\|\frac{\partial \psi}{\partial t}\right\|_{0, \alpha}$. Der Term $I_{2}$ entspricht der Ableitung des Einfachschichtpotentials mit Hölder-stetiger Dichte. Daher folgt $\left\|I_{2}\right\|_{0, \alpha} \leq c\|\psi\|_{0, \alpha}$. Der letzte Term kann unter Ausnutzung der HölderStetigkeit von $\frac{\partial \psi}{\partial t}$ im ersten Argument ähnlich wie in Lemma 4.1 aus [36] behandelt werden. Somit ist die Aussage für $p=1$ bewiesen. Für $p>1$ geht man induktiv genau wie in Korollar 4.2 aus [36] vor.

In Korollar 4.2 aus [36] wurde die Ungleichung

$$
\|u\|_{p, \alpha} \leq C \max _{j=0,1}\left\|\frac{\partial \psi}{\partial t}\right\|_{p, \infty}
$$

bewiesen. Auch diese Ungleichung könnte man für die Fehleranalyse benutzen. Betrachtet man Funktionen $\psi$ der Form $\psi(t, \tau)=k(t, \tau) \varphi(\tau)$ mit hinreichend glatter Kernfunktion $k$ und Dichte $\varphi$, so haben wir mit dem obigen Lemma nachgewiesen, daß der Operator $B_{2}(k)$ um eine Stufe glättet. Diese Eigenschaft, die man nicht aus der Ungleichung 7.3 erhält, werden wir uns bei der Fehleranalyse zunutze machen.

An dieser Stelle erfolgt ein kurzer Einschub. Falls man die Operatormatrix $M$ beim Potentialansatz mit der Aufspaltung (5.4) untersuchen will, so kann man sich die starken Glättungseigenschaften des Operators $S_{i}-S_{a}$ zunutze machen. Es gilt $S_{i}-S_{a}=B_{4}\left(k_{S_{i} S_{a}}\right)$ mit

$$
B_{4}(k) \varphi(t):=\int_{0}^{2 \pi} \ln \left(4 \sin ^{2} \frac{t-\tau}{2}\right) \sin ^{2} \frac{t-\tau}{2} k(t, \tau) \varphi(\tau) \mathrm{d} \tau,
$$

Der Kern $k_{S_{i} S_{a}}$ ist dabei ein Element aus $C^{m+1, \alpha}([0,2 \pi] \times[0,2 \pi])$. Für $B_{4}$ betrachtet man den Approximationsoperator $B_{4, n}$ mit

$$
B_{4, n}(k) \varphi(t):=\int_{0}^{2 \pi} \ln \left(4 \sin ^{2} \frac{t-\tau}{2}\right) \sin ^{2} \frac{t-\tau}{2} P_{n}(k(t, \cdot) \varphi)(\tau) \mathrm{d} \tau .
$$

Der Operator $B_{4}$ wurde in [41] in Sobolev-Räumen betrachtet. Wir werden nachweisen, daß er stärker glättet als der Operator $B_{2}$ :

Lemma 7.2. Sei $\psi \in C^{p, l, \alpha}([0,2 \pi] \times[0,2 \pi])(p \in \mathbb{N}, l:=\max (0, p-3))$, dann gilt für die Funktion

$$
v(t):=\int_{0}^{2 \pi} \ln \left(4 \sin ^{2} \frac{t-\tau}{2}\right) \sin ^{2} \frac{t-\tau}{2} \psi(t, \tau) \mathrm{d} \tau
$$

die Abschätzung

$$
\|v\|_{p, \alpha} \leq C \max _{j=0, \ldots, \min (p, 3)}\left\|\frac{\partial^{j} \psi}{\partial t^{j}}\right\|_{l, \alpha}
$$

wobei die Konstante $C$ von $p$ und $\alpha$ abhängt. 
Beweis. Für die Ableitung von $v$ ergibt sich:

$$
\begin{aligned}
v^{\prime}(t)= & \int_{0}^{2 \pi} \sin \frac{t-\tau}{2} \cos \frac{t-\tau}{2} \psi(t, \tau) \mathrm{d} \tau \\
& +\int_{0}^{2 \pi} \ln \left(4 \sin ^{2} \frac{t-\tau}{2}\right) \sin \frac{t-\tau}{2} \cos \frac{t-\tau}{2} \psi(t, \tau) \mathrm{d} \tau \\
& +\int_{0}^{2 \pi} \ln \left(4 \sin ^{2} \frac{t-\tau}{2}\right) \sin ^{2} \frac{t-\tau}{2} \frac{\partial \psi}{\partial t}(t, \tau) \mathrm{d} \tau \\
= & : I_{1}(t)+I_{2}(t)+I_{3}(t)
\end{aligned}
$$

$I_{1}$ ist ein Integral mit glattem Kern, so daß die Aussage hier einfach nachweisbar ist, $I_{3}$ hat die gleiche Struktur wie $v$, so daß nur noch $I_{2}$ zu untersuchen ist:

$$
\begin{aligned}
I_{2}^{\prime}(t)= & \int_{0}^{2 \pi} \cos ^{2} \frac{t-\tau}{2} \psi(t, \tau) \mathrm{d} \tau \\
& +\frac{1}{2} \int_{0}^{2 \pi} \ln \left(4 \sin ^{2} \frac{t-\tau}{2}\right)\left(\cos ^{2} \frac{t-\tau}{2}-\sin ^{2} \frac{t-\tau}{2}\right) \psi(t, \tau) \mathrm{d} \tau \\
& +\int_{0}^{2 \pi} \ln \left(4 \sin ^{2} \frac{t-\tau}{2}\right) \sin \frac{t-\tau}{2} \cos \frac{t-\tau}{2} \frac{\partial \psi}{\partial t}(t, \tau) \mathrm{d} \tau .
\end{aligned}
$$

Somit können wir das vorherige Lemma auf $I_{2}^{\prime}$ anwenden, woraus die Aussage für $p \leq 3$ folgt. Für größere $p$ geht man induktiv wie in Korollar 4.2 aus [36] vor.

Der Operator $B_{4}$ eröffnet also eine weitere Möglichkeit zur Fehleranalyse; darauf werden wir hier allerdings nicht näher eingehen.

Im nächsten Lemma nutzen wir folgende Approximationseigenschaft des Interpolationsoperators in Hölder-Räumen aus:

$$
\left\|P_{n} f-f\right\|_{p, \beta} \leq C \frac{\ln n}{n^{q-p+\alpha-\beta}}\|f\|_{q, \alpha} .
$$

Diese Ungleichung ist gültig für $f \in C^{q, \alpha}[0,2 \pi], 0 \leq p \leq q$ und $0<\beta<\alpha<1$ und eine Konstante $C$, die von $p, q, \alpha$ und $\beta$ abhängt (vgl. [59], Seite 40 und [60], Seite 78). Mit Hilfe dieser Ungleichung können wir die folgende Aussage beweisen:

Lemma 7.3. Sei $k \in C^{q, \alpha}([0,2 \pi] \times[0,2 \pi]), p, q \in \mathbb{N}_{0}$ mit $0 \leq p \leq q, 0<\beta<\alpha / 4<$ $1 / 4$ und

$$
\psi_{n}(t, \tau):=P_{n}(k(t, \cdot))(\tau)-k(t, \tau),
$$

dann gilt für $\psi_{n}$ folgende Abschätzung:

$$
\left\|\psi_{n}\right\|_{p, \beta} \leq \frac{C}{n^{q-p+\alpha / 2}}\|k\|_{q, \alpha}
$$


Beweis. Sei $l, j \in \mathbb{N}_{0}$ mit $j+l \leq p$ und $\frac{\partial^{j+l}}{\partial t^{j} \partial \tau^{l}} \psi_{n}(t, \tau)=: \psi_{j l}(t, \tau)$. Unter Benutzung der Gleichung $\frac{\partial^{j}}{\partial t^{j}} P_{n}(k(t, \cdot))=P_{n}\left(\frac{\partial^{j} k}{\partial t^{j}}(t, \cdot)\right)$, die aus der Lagrange-Darstellung von $P_{n}$ ersichtlich ist, erhalten wir:

$$
\begin{aligned}
\left|\psi_{j l}(t, \tau)\right| & =\left|\frac{\partial^{l}}{\partial \tau^{l}} P_{n}\left(\frac{\partial^{j} k}{\partial t^{j}}(t, \cdot)\right)(\tau)-\frac{\partial^{j+l} k}{\partial t^{j} \partial \tau^{l}}(t, \tau)\right| \\
& \leq\left\|P_{n}\left(\frac{\partial^{j} k}{\partial t^{j}}(t, \cdot)\right)-\frac{\partial^{j} k}{\partial t^{j}}(t, \cdot)\right\|_{l, \beta} \\
& \leq C \frac{\ln n}{n^{q-l-j+\alpha-\beta}}\left\|\frac{\partial^{j} k}{\partial t^{j}}(t, \cdot)\right\|_{q-j, \alpha} \\
& \leq \frac{C}{n^{q-l-j+\alpha / 2}}\|k\|_{q, \alpha} .
\end{aligned}
$$

Somit haben wir

$$
\left\|\psi_{n}\right\|_{p} \leq \frac{C}{n^{q-p+\alpha / 2}}\|k\|_{q, \alpha}
$$

bewiesen. Nun zur Hölder-Stetigkeit von $\psi_{n}$. Seien hierzu $t_{1}, t_{2}, \tau_{1}$ und $\tau_{2} \in[0,2 \pi]$ und $l+j=p$.

$$
\begin{aligned}
\left|\psi_{j l}\left(t_{1}, \tau_{1}\right)-\psi_{j l}\left(t_{2}, \tau_{2}\right)\right| & \left|\psi_{j l}\left(t_{1}, \tau_{1}\right)-\psi_{j l}\left(t_{2}, \tau_{1}\right)\right|+\left|\psi_{j l}\left(t_{2}, \tau_{1}\right)-\psi_{j l}\left(t_{2}, \tau_{2}\right)\right| \\
= & \mid \frac{\partial^{l}}{\partial \tau^{l}} P_{n}\left(\frac{\partial^{j} k}{\partial t^{j}}\left(t_{1}, \cdot\right)-\frac{\partial^{j} k}{\partial t^{j}}\left(t_{2}, \cdot\right)\right)\left(\tau_{1}\right)- \\
& \quad \frac{\partial^{j+l}}{\partial t^{j} \partial \tau^{l}}\left(k\left(t_{1}, \tau_{1}\right)-k\left(t_{2}, \tau_{1}\right)\right) \mid \\
& +\mid \frac{\partial^{l}}{\partial \tau^{l}}\left(P_{n}\left(\frac{\partial^{j} k}{\partial t^{j}}\left(t_{2}, \cdot\right)\right)\left(\tau_{1}\right)-P_{n}\left(\frac{\partial^{j} k}{\partial t^{j}}\left(t_{2}, \cdot\right)\right)\left(\tau_{2}\right)\right) \\
\leq & C \frac{\ln n}{n^{q-l-j+3 \alpha / 4-\beta}} \| \frac{\partial^{j}}{\partial t^{j}}\left(k\left(t_{1}, \cdot\right)-k\left(t_{2}, \cdot\right)\right)||_{q-j, 3 \alpha / 4} \\
& +\left|\frac{\partial^{l}}{\partial \tau^{l}} P_{n}\left(\frac{\partial^{j} k}{\partial t^{j}}\left(t_{2}, \cdot\right)\right)-\frac{\partial^{j+l} k}{\partial t^{j} \partial \tau^{l}}\left(t_{2}, \cdot\right)\right|_{\beta}\left|\tau_{1}-\tau_{2}\right|^{\beta} \\
\leq & \frac{C}{n^{q-p+\alpha / 2}}\left\|k\left(t_{1}, \cdot\right)-k\left(t_{2}, \cdot\right)\right\|_{q, 3 \alpha / 4}+\frac{C}{n^{q-p+\alpha / 2}}\|k\|_{q, \alpha}\left|\tau_{1}-\tau_{2}\right|^{\beta} .
\end{aligned}
$$

Nun ist noch der Nachweis $\left\|k\left(t_{1}, \cdot\right)-k\left(t_{2}, \cdot\right)\right\|_{q, 3 \alpha / 4} \leq C\|k\|_{q, \alpha}\left|t_{1}-t_{2}\right|^{\beta}$ zu führen. Der Beweis der Ungleichung

$$
\left\|k\left(t_{1}, \cdot\right)-k\left(t_{2}, \cdot\right)\right\|_{q} \leq\|k\|_{q, \alpha}\left|t_{1}-t_{2}\right|^{\alpha} \leq C\|k\|_{q, \alpha}\left|t_{1}-t_{2}\right|^{\beta}
$$


ist nicht schwierig, etwas komplizierter ist die Situation für die Hölder-Halbnorm. Sei hierzu $k_{j l}:=\partial^{j+l} k / \partial t^{j} \partial \tau^{l}$ für $j, l \in \mathbb{N}_{0}$ und $j+l=q$. Wir verwenden einen Trick, der auch zum Nachweis der kompakten Einbettung von Hölder-Räumen verwendet wird (vgl. [37], Theorem 7.4). Seien $\tilde{\tau}, \hat{\tau} \in[0,2 \pi]$.

$$
\begin{aligned}
& \left|k_{j l}\left(t_{1}, \tilde{\tau}\right)-k_{j l}\left(t_{2}, \tilde{\tau}\right)-\left(k_{j l}\left(t_{1}, \hat{\tau}\right)-k_{j l}\left(t_{2}, \hat{\tau}\right)\right)\right| \\
& =\left|k_{j l}\left(t_{1}, \tilde{\tau}\right)-k_{j l}\left(t_{2}, \tilde{\tau}\right)-\left(k_{j l}\left(t_{1}, \hat{\tau}\right)-k_{j l}\left(t_{2}, \hat{\tau}\right)\right)\right|^{1 / 4} \\
& \quad\left|k_{j l}\left(t_{1}, \tilde{\tau}\right)-k_{j l}\left(t_{2}, \tilde{\tau}\right)-\left(k_{j l}\left(t_{1}, \hat{\tau}\right)-k_{j l}\left(t_{2}, \hat{\tau}\right)\right)\right|^{3 / 4} \\
& \leq\left(2|| k_{j l}||_{\alpha}\left|t_{1}-t_{2}\right|^{\alpha}\right)^{1 / 4}\left(2|| k_{j l}||_{\alpha}|\tilde{\tau}-\hat{\tau}|^{\alpha}\right)^{3 / 4} \\
& \leq C|| k_{j l}||_{\alpha}\left|t_{1}-t_{2}\right|^{\alpha / 4}|\tilde{\tau}-\hat{\tau}|^{3 \alpha / 4}
\end{aligned}
$$

Somit folgt

$$
\left\|k\left(t_{1}, \cdot\right)-k\left(t_{2}, \cdot\right)\right\|_{q, 3 \alpha / 4} \leq C\|k\|_{q, \alpha}\left|t_{1}-t_{2}\right|^{\beta}
$$

und daher die Behauptung.

Nun verfügen wir über alle Hilfsmittel, die wir für die Fehleranalyse benötigen. Wir betrachten jetzt wieder das Beispiel zum Transmissionsproblem. Der folgende Satz ist ähnlich zu Theorem 4.4 aus [36] bzw. Satz 4.3 aus [24]. Allerdings wurde der Beweis dort für analytische bzw. unendlich oft differenzierbare Ränder geführt, während wir hier weiterhin nur $C^{m+2, \alpha}$-Glätte voraussetzen.

Für $q, p \in \mathbb{N}_{0}, 0<\delta, \gamma<1$ betrachten wir den Raum

$$
C^{q, p, \delta, \gamma}:=\left(C^{q, \delta}[0,2 \pi] \times C^{p, \gamma}[0,2 \pi]\right)^{2} .
$$

Die Norm in diesem Raum bezeichnen wir mit $\|\cdot\|_{q, p, \delta, \gamma}$. Falls $\delta=\gamma$ bezeichnen wir den Raum mit $C^{q, p, \delta}$ und die Norm mit $\|\cdot\|_{q, p, \delta}$.

Satz 7.4. Es existiert ein $\epsilon>0$, so daß für hinreichend große $n \in \mathbb{N}$ die Näherungsgleichung (7.1) eine eindeutige Lösung $\varphi_{n}$ besitzt. Der Abstand von $\varphi_{n}$ zur wahren Lösung $\varphi$ genügt der Abschätzung

$$
\left\|\varphi_{n}-\varphi\right\|_{2,1, \epsilon} \leq \frac{C}{n^{m-2+\epsilon}}\left(\|f\|_{m, m-1, \alpha}+\|\varphi\|_{m, m-1, \alpha}\right), \quad C=C(\alpha, \epsilon, m, \Gamma) .
$$

Für die Approximation $u_{c, n}$ an die Cauchy-Daten $u_{c}$ von $u_{0}$ gilt weiterhin

$$
\left\|u_{c, n}-u_{c}\right\|_{2,1, \epsilon} \leq \frac{C}{n^{m-2+\epsilon}}\left(\|f\|_{m, m-1, \alpha}+\|\varphi\|_{m, m-1, \alpha}\right), \quad C=C(\alpha, \epsilon, m, \Gamma) .
$$

Beweis. Sei $q \in \mathbb{N}, \beta_{1}, \beta_{2}, \beta_{3}, \beta_{4} \in \mathbb{R}_{+}$mit $0<\beta_{j}<\beta_{j+1} / 4, j=1,2,3$ und $\beta_{4}<\alpha / 4$.

Zunächst wollen wir zeigen, daß die Operatorfolge $P_{n} M_{k, n}$ in $\operatorname{der} C^{2,1, \beta_{1}, \beta_{3}}$-Norm gegen $M_{k}$ konvergiert. Betrachten wir den Operator $K$ (der Beweis ist für $K_{1, i}, K_{1, a}$, $K_{2, i}$ und $K_{2, a}$ gleich, wir verzichten daher auf den Index) und die zugehörigen Operatoren $B_{1}\left(k_{K}\right)$ und $B_{2}\left(w_{K}\right)$. Die Beweise für den Operator $B_{1}$ sind einfacher, da er 
einen $C^{m, \alpha}$-glatten Kern hat, während $B_{2}$ eine logarithmische Singularität besitzt. Wir betrachten im folgenden daher nur $B_{2}$. Sei

$$
\psi_{n}(t, \tau):=P_{n}\left(w_{K}(t, \cdot) \varphi\right)(\tau)-w_{K}(t, \tau) \varphi(\tau),
$$

dann folgt aus Lemma 7.1 und Lemma 7.3 für $2 \leq q \leq m$ :

$$
\begin{aligned}
\left\|\left(B_{2, n}\left(w_{K}\right)-B_{2}\left(w_{K}\right)\right) \varphi\right\|_{2, \beta_{1}} & \leq C \max _{j=0,1}\left\|\frac{\partial^{j}}{\partial t^{j}} \psi_{n}\right\|_{1, \beta_{1}} \\
& \leq \frac{C}{n^{q-2+\beta_{2} / 2}} \max _{j=0,1}\left\|\frac{\partial^{j}}{\partial t^{j}} w_{K} \varphi\right\|_{q-1, \beta_{2}} \\
& \leq \frac{C}{n^{q-2+\beta_{2} / 2}}\|\varphi\|_{q-1, \beta_{2}} .
\end{aligned}
$$

Für den Operator $B_{2, n}\left(w_{K}\right)$ ergibt sich:

$$
\begin{aligned}
\left\|B_{2, n}\left(w_{K}\right) \varphi\right\|_{q, \beta_{2}} & \leq\left\|\left(B_{2, n}\left(w_{K}\right)-B_{2}\left(w_{K}\right)\right) \varphi\right\|_{q, \beta_{2}}+\left\|B_{2}\left(w_{K}\right) \varphi\right\|_{q, \beta_{2}} \\
& \leq C \max _{j=0,1}\left(\left\|\frac{\partial^{j}}{\partial t^{j}} \psi_{n}\right\|_{q-1, \beta_{2}}+\left\|\frac{\partial^{j}}{\partial t^{j}} w_{k} \varphi\right\|_{q-1, \beta_{2}}\right) \\
& \leq C\|\varphi\|_{q-1, \beta_{3}} .
\end{aligned}
$$

Aus der letzten Ungleichung erhalten wir

$$
\begin{aligned}
\left\|\left(P_{n} B_{2, n}\left(w_{K}\right)-B_{2, n}\left(w_{K}\right)\right) \varphi\right\|_{2, \beta_{1}} & \leq C \frac{\ln n}{n^{q-2+\beta_{2}-\beta_{1}}}\left\|B_{2, n}\left(w_{K}\right) \varphi\right\|_{q, \beta_{2}} \\
& \leq \frac{C}{n^{q-2+\beta_{2} / 2}}\|\varphi\|_{q-1, \beta_{3}} .
\end{aligned}
$$

Setzt man diese Ungleichungen zusammen, ergibt sich

$$
\left\|\left(P_{n} B_{2, n}\left(w_{K}\right)-B_{2}\left(w_{K}\right)\right) \varphi\right\|_{2, \beta_{1}} \leq \frac{C}{n^{q-2+\beta_{2} / 2}}\|\varphi\|_{q-1, \beta_{3}} \leq \frac{C}{n^{q-2+\beta_{1}}}\|\varphi\|_{q, \beta_{1}} .
$$

Ähnlich zeigt man die folgenden Ungleichungen:

$$
\begin{aligned}
\left\|\left(P_{n} B_{2, n}\left(w_{S}\right)-B_{2}\left(w_{S}\right)\right) \varphi\right\|_{2, \beta_{1}} & \leq \frac{C}{n^{q-2+\beta_{1}}}\|\varphi\|_{q-1, \beta_{3}}, \\
\left\|\left(P_{n} B_{2, n}\left(w_{K^{*}}\right)-B_{2}\left(w_{K^{*}}\right)\right) \varphi\right\|_{1, \beta_{3}} & \leq \frac{C}{n^{q-2+\beta_{1}}}\|\varphi\|_{q-1, \beta_{3}}, \\
\left\|\left(P_{n} B_{2, n}\left(w_{T_{i} T_{a}}\right)-B_{2}\left(w_{T_{i} T_{a}}\right)\right) \varphi\right\|_{1, \beta_{3}} & \leq \frac{C}{n^{q-2+\beta_{1}}}\|\varphi\|_{q, \beta_{1}}
\end{aligned}
$$

Setzt man alle Ungleichungen zusammen, so erhalten wir

$$
\left\|\left(P_{n} M_{k, n}-M_{k}\right) \varphi\right\|_{2,1, \beta_{1}, \beta_{3}} \leq \frac{C}{n^{q-2+\beta_{1}}}\|\varphi\|_{q, q-1, \beta_{1}, \beta_{3}}
$$


und damit die Normkonvergenz in der $C^{2,1, \beta_{1}, \beta_{3}}$-Norm. Analog zu Bemerkung 5.5 zeigt man die Invertierbarkeit des Operators $M_{d}+M_{k}$ in $\operatorname{der} C^{2, \beta_{1}, \beta_{3}}$ Norm. Der Nachweis der Abschätzung

$$
\left\|\varphi_{n}-\varphi\right\|_{2,1, \beta_{1}, \beta_{3}} \leq C\left(\left\|P_{n} f-f\right\|_{2,1, \beta_{1}, \beta_{3}}+\left\|\left(P_{n} M_{k, n}-M_{k}\right) \varphi\right\|_{2,1, \beta_{1}, \beta_{3}}\right)
$$

ist dann Standardstoff der Funktionalanalysis (vgl. z.B. [36], Theorem 4.4). Aus den Ungleichungen (7.4) und (7.7) folgt die Ungleichung

$$
\left\|\varphi_{n}-\varphi\right\|_{2,1, \beta_{1}, \beta_{3}} \leq \frac{C}{n^{m-2+\epsilon}}\left(\|f\|_{m, m-1, \alpha}+\|\varphi\|_{m, m-1, \alpha}\right)
$$

und damit insbesondere die Abschätzung (7.5).

Nun zu den Cauchy-Daten. Es gilt

$$
\begin{aligned}
u_{c}-u_{c, n} & =\left(Q_{d}+Q_{k}\right) \varphi-\left(Q_{d}+P_{n} Q_{k, n}\right) \varphi_{n} \\
& =\left(Q_{d}+P_{n} Q_{k, n}\right)\left(\varphi-\varphi_{n}\right)+\left(Q_{k}-P_{n} Q_{k}\right) \varphi .
\end{aligned}
$$

Jetzt untersuchen wir die Komponenten von $Q_{k}$ bzw. $Q_{k, n}$. Neu im Vergleich zu $M_{k}$ sind die Operatoren $B_{1}\left(k_{T}\right), B_{2}\left(w_{T}\right)$ und $B_{3}$. Die ersten beiden Operatoren können analog zu den vorherigen behandelt werden. Der Operator $B_{3}$ entspricht, wie bereits erwähnt, dem Operator $T_{0}$ auf dem Einheitskreis entspricht. Zusätzlich wählen wir nun Konstanten $\epsilon, \beta_{0} \in \mathbb{R}_{+}$mit $\epsilon<\beta_{0} / 4$ und $\beta_{0}<\beta_{1} / 4$. Aus den Abbildungseigenschaften von $T_{0}$ folgt

$$
\left\|\left(B_{3}-B_{3, n}\right) \varphi\right\|_{1, \epsilon} \leq\left\|\varphi-P_{n} \varphi\right\|_{2, \epsilon} \leq \frac{C \ln n}{n^{q-2+\beta_{1}-\epsilon}}\|\varphi\|_{q, \beta_{1}} \leq \frac{C}{n^{q-2+\epsilon}}\|\varphi\|_{q, \beta_{1}} .
$$

Zudem gilt $P_{n} B_{3, n}=B_{3, n}$ (vgl. z.B. [36], Gleichung (3.6)), so daß wir insgesamt die Abschätzung

$$
\left\|\left(P_{n} Q_{k, n}-Q_{k}\right) \varphi\right\|_{2,1, \epsilon} \leq \frac{C}{n^{q-2+\epsilon}}\|\varphi\|_{q, q-1, \beta_{1}}
$$

erhalten. Nun folgt die Aussage aus den bereits bewiesenen Ungleichungen.

Falls die Gebiete analytisch sind, kann nachgewiesen werden, daß die Konvergenz der Näherungslösung gegen die wahre Lösung exponentiell ist (vgl. z.B. [37], Theorem 11.7).

Der Konvergenzbeweis benutzt keine Eigenschaften des konkret betrachteten Gebiets und kann somit für beliebige Gebietskonstellationen verwendet werden.

Das Näherungsverfahren für den Greensche Ansatz wurde unter Verwendung von Sobolev-Räumen in [28] analysiert. Dort wurde nur die Situation eines einzigen Randes untersucht. Diese Konvergenzanalyse kann mit den hier bereitgestellten Hilfsmitteln leicht auf Hölder-Räume und mehrere Gebiete übertragen werden. Auch der Ansatz von Kleinman und Martin kann in ähnlicher Weise untersucht werden. In [41] wurde ein Einfachschichtpotentialansatz beim Dirichlet-Problem untersucht (vgl. zudem [37], Kapitel 13.4). Dies kann auch auf das Transmisionsproblem übertragen werden. Auf 

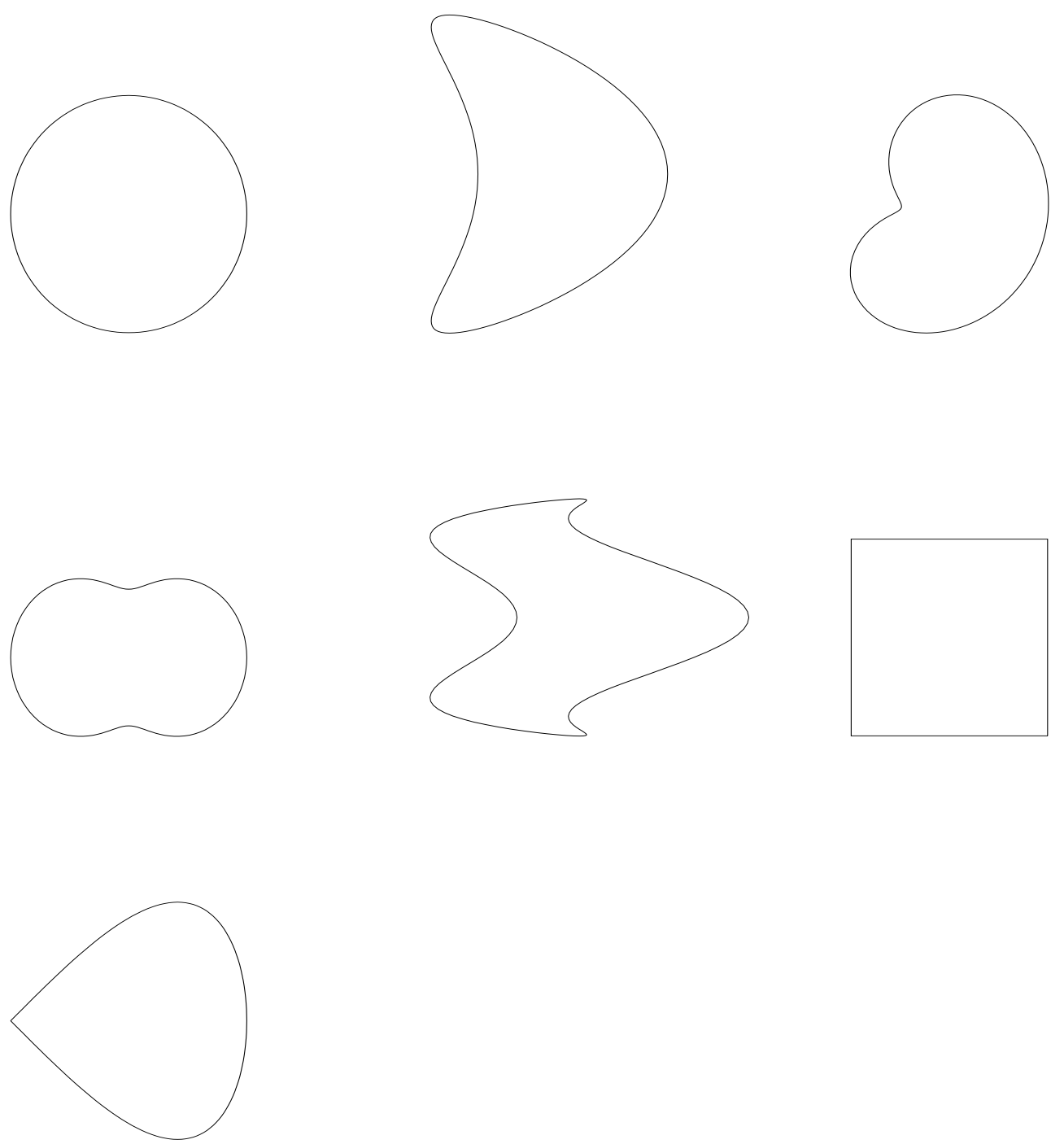

Abbildung 7.2: Verschiedene Gebiete: Kreis, Drache, Bohne, Erdnuß, Raumschiff, Quadrat, Tropfen 
weitere Details wollen wir jedoch verzichten und nur noch ein Verfahren zur Lösung der auftretenden Gleichungssysteme angeben.

Die Darstellung orientiert sich an [17], Abschnitt 3.4.7. Diskretisiert man die Operatormatrix $M$ bzgl. $n$ Knotenpunkten auf den Rändern $\Gamma_{j}, j=1, \ldots, L$, so erhält man eine Matrix $A=\left(A_{j k}\right) \in \mathbb{C}^{L \times L}$ mit $A_{j k} \in \mathbb{C}^{n \times n}$ für $j, k=1, \ldots, L$. Wir werden stets davon ausgehen, daß die auftretenden Matrizen auf der Diagonale der Blockmatrix nach eventueller Pivotisierung in eine linke untere und eine rechte obere invertierbare Dreiecksmatrix zerlegt werden können. Im ersten Schritt ergibt sich dann folgende Vorgehensweise:

1. Bestimme für eine geeignete Permutationsmatrix $P_{1} \in \mathbb{R}^{n \times n}$ eine LU-Zerlegung von $P_{1} A_{11}$, d.h. $P_{1} A_{11}=L_{11} U_{11}$.

2. Für $j=2, \ldots, L$ bestimme man Matrizen $U_{1 j}$ und $L_{j 1}$ mit $P_{1} A_{1 j}=L_{11} U_{1 j}$ und $A_{j 1}=L_{j 1} U_{11}$. Die Matrizen werden hierbei durch Vorwärtseinsetzen bestimmt, wobei zur Berechnung von $L_{j 1}$ das transponierte System $A_{j 1}^{t}=U_{11}^{t} L_{j 1}^{t}$ verwendet wird.

3. Für $j, k=2, \ldots, L$ sei $A_{j k}^{(1)}:=A_{j k}-L_{j 1} U_{1 k}$.

Somit ergibt sich:

$$
\begin{aligned}
& \left(\begin{array}{cccc}
P_{1} & 0 & \ldots & 0 \\
0 & I & \ldots & 0 \\
\vdots & & \ddots & \vdots \\
0 & & \ldots & I
\end{array}\right)\left(\begin{array}{cccc}
A_{11} & \ldots & \ldots & A_{1 n} \\
\vdots & \ddots & & \vdots \\
\vdots & & \ddots & \vdots \\
A_{n 1} & \ldots & \ldots & A_{n n}
\end{array}\right) \\
& =\left(\begin{array}{cccc}
L_{11} U_{11} & L_{11} U_{12} & \ldots & L_{11} U_{1 n} \\
L_{21} U_{11} & A_{22}^{(1)}+L_{21} U_{12} & \ldots & A_{2 n}^{(1)}+L_{21} U_{1 n} \\
\vdots & & \ddots & \vdots \\
L_{n 1} U_{11} & A_{n 2}^{(1)}+L_{n 1} U_{12} & \ldots & A_{n n}^{(1)}+L_{n 1} U_{1 n}
\end{array}\right) \\
& =\left(\begin{array}{cccc}
L_{11} & 0 & \ldots & 0 \\
L_{21} & I & \ldots & 0 \\
\vdots & & \ddots & \vdots \\
L_{n 1} & & \ldots & I
\end{array}\right)\left(\begin{array}{cccc}
I & 0 & \ldots & 0 \\
0 & A_{22}^{(1)} & \ldots & A_{2 n}^{(1)} \\
\vdots & & \ddots & \vdots \\
0 & A_{n 2}^{(1)} & \ldots & A_{n n}^{(1)}
\end{array}\right)\left(\begin{array}{cccc}
U_{11} & U_{12} & \ldots & U_{1 n} \\
0 & I & \ldots & 0 \\
\vdots & & \ddots & \vdots \\
0 & & \ldots & I
\end{array}\right) .
\end{aligned}
$$

Sei nun $P_{2}$ eine Permutationsmatrix, die eine LU-Zerlegung für $A_{22}^{(1)}$ ermöglicht: 
$P_{2} A_{22}^{(1)}=L_{22} U_{22}$, dann ergibt sich:

$$
\begin{aligned}
& \left(\begin{array}{cccc}
P_{1} & 0 & \ldots & 0 \\
0 & P_{2} & \ldots & 0 \\
\vdots & & \ddots & \vdots \\
0 & & \ldots & I
\end{array}\right)\left(\begin{array}{cccc}
A_{11} & \ldots & \ldots & A_{1 n} \\
\vdots & \ddots & & \vdots \\
\vdots & & \ddots & \vdots \\
A_{n 1} & \ldots & \ldots & A_{n n}
\end{array}\right) \\
= & \left(\begin{array}{cccc}
L_{11} & 0 & \ldots & 0 \\
P_{2} L_{21} & I & \ldots & 0 \\
\vdots & & \ddots & \vdots \\
L_{n 1} & & \ldots & I
\end{array}\right)\left(\begin{array}{cccc}
I & 0 & \ldots & 0 \\
0 & L_{22} U_{22} & \ldots & P_{2} A_{2 n}^{(1)} \\
\vdots & & \ddots & \vdots \\
0 & A_{n 2}^{(1)} & \ldots & A_{n n}^{(1)}
\end{array}\right)\left(\begin{array}{cccc}
U_{11} & U_{12} & \ldots & U_{1 n} \\
0 & I & \ldots & 0 \\
\vdots & & \ddots & \vdots \\
0 & & \ldots & I
\end{array}\right) .
\end{aligned}
$$

Somit kann die Blockmatrix $A$ (bzw. eine geeignete Permutation von $A$ ) in eine linke untere und eine rechte obere Blockmatrix zerlegt werden. Die auftretenden Gleichungssysteme können also durch Vor- und Rückwärtseinsetzen gelöst werden.

Bemerkung 7.5. Wenn nichts anderes erwähnt ist, verwenden wir bei den numerischen Beispielen die folgenden Parameter. Bei nur einem Rand sind die Wellenzahlen gegeben durch $\kappa_{0}=1.9$ und $\kappa_{1}=1.0$ und für die Übergangskonstante gilt $\rho=0.7$. Wir verwenden 64 Knotenpunkte auf dem Einheitskreis zur Diskretisierung des Fernfeldes und einen Potentialansatz zur Lösung des direkten Problems. Folgende Parametrisierungen werden untersucht:

$$
\begin{aligned}
\text { Kreis: } z(t) & =\left(\begin{array}{l}
\cos t \\
\sin t
\end{array}\right), \\
\text { Bohne: } z(t) & =\frac{1+.9 \cos t+.1 \sin 2 t}{1+.75 \cos t}\left(\begin{array}{c}
\cos t \\
\sin t
\end{array}\right), \\
\text { Erdnuß: } z(t) & =\sqrt{\cos ^{2} t+.25 \sin ^{2} t}\left(\begin{array}{c}
\cos t \\
\sin t
\end{array}\right), \\
\text { Drache: } z(t) & =\left(\begin{array}{cc}
\cos t+0.65(\cos 2 t-1) \\
1.5 \sin t
\end{array}\right), \\
\text { Raumschiff: } z(t) & =\left(\begin{array}{cc}
e^{\cos t}+.5 \cos (4 t)-2 \\
\sin t
\end{array}\right), \\
\text { Quadrat: } z(t) & =\left\{\begin{array}{cc}
(.25 \pi,-.25 \pi+t) & t \in[0, .5 \pi], \\
(-.25 \pi, 1.25 \pi-t) & t \in[\pi, 1.5 \pi], \\
(-1.75 \pi+t,-.25 \pi) & t \in[1.5 \pi, 2 \pi]
\end{array}\right. \\
\text { Tropfen: } z(t) & =\left(\begin{array}{c}
2 \sin (t / 2) \\
-\sin t
\end{array}\right) .
\end{aligned}
$$

Die numerischen Experimente wurden auf einer Compaq XP1000 Workstation durchgeführt. Es wurde stets mit doppelter Genauigkeit (d.h. bis 10-16) gerechnet. 


\begin{tabular}{lcccccc}
\hline \hline $\mathrm{n}$ & Kreis & Drache & Raumschiff & Tropfen & Quadrat & Zeit(sec) \\
\hline 16 & $1.2 \mathrm{e}-12$ & 0.005 & 0.07 & 0.02 & 0.05 & 0 \\
32 & $4.5 \mathrm{e}-16$ & $1.0 \mathrm{e}-06$ & 0.01 & 0.01 & 0.03 & 0 \\
64 & $4.2 \mathrm{e}-16$ & $2.5 \mathrm{e}-12$ & $8.8 \mathrm{e}-5$ & 0.006 & 0.02 & 0 \\
128 & $2.4 \mathrm{e}-16$ & $4.3 \mathrm{e}-16$ & $1.6 \mathrm{e}-6$ & 0.004 & 0.01 & 0 \\
256 & $5.5 \mathrm{e}-16$ & $5.6 \mathrm{e}-17$ & $1.7 \mathrm{e}-9$ & 0.002 & 0.007 & 3 \\
512 & $1.0 \mathrm{e}-15$ & $7.6 \mathrm{e}-16$ & $2.4 \mathrm{e}-15$ & 0.001 & 0.004 & 35 \\
\hline \hline
\end{tabular}

Tabelle 7.1: Konvergenzgeschindigkeit bei verschiedenen Gebieten

\begin{tabular}{lcccc}
\hline \hline $\mathrm{n}$ & Pot & Zeit & Pot(S) & Zeit \\
\hline 16 & 0.07 & 0 & 0.16 & 0 \\
32 & 0.01 & 0 & 0.02 & 0 \\
64 & $8.8 \mathrm{e}-5$ & 0 & 0.002 & 0 \\
128 & $1.6 \mathrm{e}-5$ & 0 & $4.7 \mathrm{e}-5$ & 0 \\
256 & $1.7 \mathrm{e}-9$ & 3 & $3.9 \mathrm{e}-8$ & 3 \\
512 & $2.4 \mathrm{e}-15$ & 36 & $3.6 \mathrm{e}-14$ & 35 \\
1024 & $6.1 \mathrm{e}-16$ & 316 & $8.3 \mathrm{e}-16$ & 313 \\
\hline \hline
\end{tabular}

\begin{tabular}{lcccccc}
\hline \hline $\mathrm{n}$ & Green & Zeit & Green(S) & Zeit & KlMa & Zeit(sec) \\
\hline 16 & 0.5 & 0 & 0.1 & 0 & 0.5 & 0 \\
32 & 0.4 & 0 & 0.01 & 0 & 0.3 & 0 \\
64 & 0.1 & 0 & 0.003 & 0 & 0.4 & 0 \\
128 & 0.006 & 0 & $8.0 \mathrm{e}-5$ & 0 & 0.01 & 0 \\
256 & $9.9 \mathrm{e}-6$ & 3 & $6.6 \mathrm{e}-8$ & 3 & $1.7 \mathrm{e}-5$ & 2 \\
512 & $1.6 \mathrm{e}-11$ & 36 & $5.8 \mathrm{e}-14$ & 33 & $2.7 \mathrm{e}-11$ & 15 \\
1024 & $7.8 \mathrm{e}-15$ & 316 & $2.0 \mathrm{e}-16$ & 313 & $2.7 \mathrm{e}-14$ & 128 \\
\hline \hline
\end{tabular}

Tabelle 7.2: Konvergenzgeschindigkeit bei verschiedenen Lösungsverfahren; Gebiet ist das Raumschiff; 
Nun untersuchen wir zunächst die Konvergenz unseres Verfahrens bei nur einem Rand. In der Tabelle 7.1 betrachten wir Kreis, Drache, Raumschiff, Tropfen und Quadrat. Als Randwerte haben wir die Abbildung $f$ mit

$$
f(x)=\left(\left(\begin{array}{c}
J_{0}\left(\kappa_{1}|x|\right)-H_{0}\left(\kappa_{0}|x|\right) \\
\left(\kappa_{0} H_{1}\left(\kappa_{0}|x|\right)-\frac{1}{\rho} \kappa_{1} J_{1}\left(\kappa_{1}|x|\right)\right) \frac{x \cdot \nu(x)}{|x|}
\end{array}\right)\right.
$$

verwendet. Aus der Eindeutigkeit des Transmissionsproblems für die gewählten Konstanten $\kappa_{0}, \kappa_{1}$ und $\rho$ folgt, daß das äußere Feld $u_{0}$ gerade durch $u_{0}(x)=H_{0}\left(\kappa_{0}|x|\right)$ und das innere Feld $u_{1}$ durch $u_{1}(x)=J_{0}\left(\kappa_{1}|x|\right)$ gegeben ist (hierbei nutzen wir aus, daß der Nullpunkt stets im Inneren der Gebiete liegt). Das Fernfeld von $H_{0}\left(\kappa_{0}|x|\right)$ kann explizit berechnet werden, es ergibt sich

$$
u_{\infty}(\hat{x})=\sqrt{\frac{2}{\kappa_{0} \pi}} e^{-\frac{i}{4 \pi}}
$$

In der Tabelle 7.1 vergleichen wir den Absolutbetrag der Näherungslösung des Fernfeldes mit der exakten Lösung an der Stelle $\hat{x}=(1,0)$. Bei dem Quadrat wurde ein Einfachschichtpotentialansatz, sonst der in Kapitel 5 beschriebene Potentialansatz verwendet. Da wir Gebiete mit Ecken nicht theoretisch untersucht haben, sind die hier aufgeführten Beispiele für das Quadrat und den Tropfen rein experimenteller Natur. Die Ergebnisse deuten jedoch darauf hin, daß sich die betrachteten Algorithmen auch auf Gebiete mit Ecken ausdehnen lassen. Bei Kreis, Drache und Raumschiff ist die Konvergenz exponentiell, allerdings zeigt sich beim Raumschiff, daß schon eine recht große Anzahl von

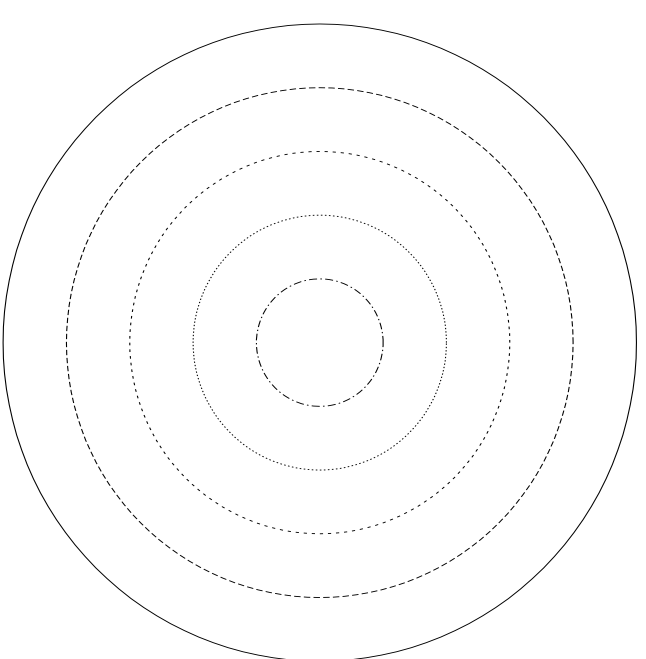

Abbildung 7.3: Geometrie 1 Knotenpunkte nötig ist, um einen hinreichend kleinen Fehler zu erhalten. Die Konvergenz bei Gebieten mit Ecken ist sehr schlecht. Hier müßte man eigentlich mit Verfahren arbeiten, die der Existenz von Ecken Rechnung tragen (vgl. z.B. [7], Kapitel 3.5, Seite 72ff.), Im Kontext von inversen Problemen kann man allerdings davon ausgehen, daß die Meßdaten sowieso fehlerbehaftet sind, so daß das Lösungsverfahren für das direkte Problem nicht allzu genau sein muß.

In Tabelle 7.2 vergleichen wir verschiedene Lösungsverfahren für das direkte Problem. Als Gebiet haben wir das Raumschiff verwendet. Als Randwerte haben wir auch hier die Funktion (7.9) verwendet. Neben dem in Kapitel 5 besprochenen Potentialansatz verwenden wir auch einen Potentialansatz, der nur auf Einfachschichtpotentialen beruht. In gleicher Weise führen wir dies beim Greenschen Ansatz durch. Neben dem 

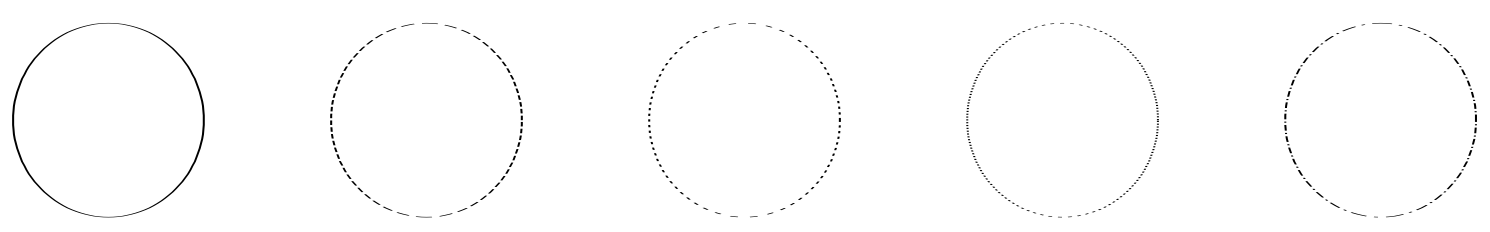

Abbildung 7.4: Geometrie 2

besprochenen Ansatz haben wir auch hier einen Ansatz implementiert, der nur auf Einfachschichtpotentialen beruht (vgl. hierzu [34], Abschnitt 4.2). Als letzte Variante verwenden wir den Ansatz von Kleinman und Martin. Für eine Untersuchung dieses Ansatzes sei auch auf die Diplomarbeit [15] verwiesen. Im Beweis von Satz 5.9 haben wir gesehen, daß sich die Komponenten der Operatormatrix $M$ bei diesem Verfahren durch eine Verknüpfung von Operatoren ergeben. Bei einem Rand werden daher beim volldiskreten System gerade 2 Matrizenmultiplikationen benötigt. Zur Effizienzsteigerung kann man hier statt der gewöhnlichen Matrizenmultiplikation das Verfahren von Strassen verwenden (vgl. [17], Abschnitt 1.3.7). Diese Methode haben wir implementiert.

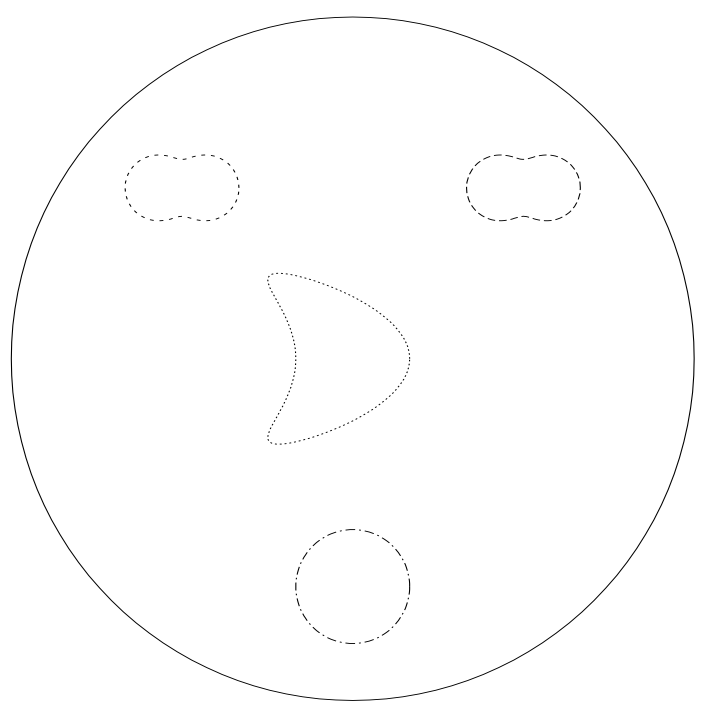

Abbildung 7.5: Geometrie 3 Wir wollen nun den Aufwand für das Lösen der Gleichungssysteme vergleichen. Wir betrachten nur die benötigten Multiplikationen. Beim Potentialansatz (wie auch beim Greenschen Ansatz) wird der Aufwand durch die LUZerlegung bestimmt. Bei $n$ Knotenpunkten hat die zu zerlegende Matrix die Größe $2 n \times 2 n$ und somit benötigt man ungefähr $\frac{1}{3}(2 n)^{3}=\frac{8}{3} n^{3}$ Multiplikationen. Beim Verfahren von Kleinman und Martin benötigt man für die LUZerlegung $n^{3} / 3$ Multiplikationen und $2 n^{3}$ Multiplikationen für die Matrizenmultiplikationen. Beim Verfahren von Strassen benötigt man für eine Matrixmultiplikation $n^{\log _{2} 7} \approx n^{2.807}$ Multiplikationen. Diese Zeitersparnis spiegelt

sich in der Tabelle 7.2 wieder. Für große Knotenzahlen ist das Verfahren von Kleinman und Martin daher erheblich schneller. Die Methode von Strassen ist rekursiv und deshalb nur für große Matrizen sinnvoll. Wir haben sie erst ab 256 Knotenpunkten verwendet. Trotz der Zeitersparnis beim Verfahren von Kleinman und Martin haben wir bei mehreren Rändern nur den Potential- und den Greenschen Ansatz implementiert, da uns das Verfahren von Kleinman und Martin hier zu kompliziert erschien (vgl. den Algorithmus in Kapitel 5).

Nun wollen wir uns einigen Beispielen mit mehreren Rändern zuwenden. Zunächst betrachten fünf ineinanderliegende Kreise (vgl. Abbildung 7.3). Für die Radien $r_{j}$ der 
Kreise $K_{j}$ gilt $r_{j}=6-j, j=1, \ldots, 5$. Die Wellenzahlen sind hier gegeben durch $\kappa_{0}=1.9, \kappa_{1}=\kappa_{3}=\kappa_{5}=1.0$ und $\kappa_{2}=\kappa_{4}=0.8$. Für die Übergangskonstanten wurde $\rho_{1}=\rho_{3}=\rho_{5}=0.7$ und $\rho_{2}=\rho_{4}=0.5$ gewählt. Das zweite Experiment mit dieser Geometrie wurde mit komplexen Wellenzahlen durchgeführt. Hier haben wir die Wellenzahlen folgendermaßen gewählt: $\kappa_{0}=1.9+i 0.5, \kappa_{1}=1.0+i 0.6, \kappa_{2}=0.8+i 0.7$, $\kappa_{3}=1.0+i 0.8, \kappa_{4}=0.8+i 0.9$ und $\kappa_{5}=1.0+i 1.0$.

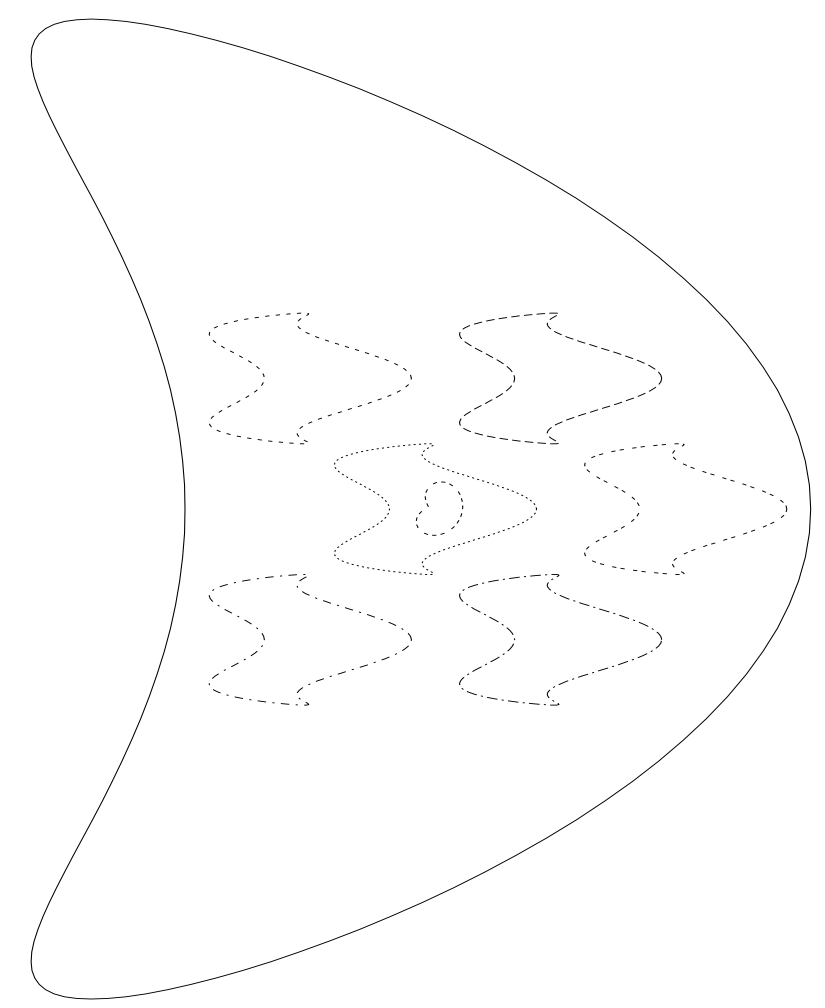

Abbildung 7.6: Geometrie 4

Daneben untersuchen wir fünf nebeneinanderliegende Kreise (vgl. Abbildung 7.4), alle mit Radius Eins. Die Mittelpunkte der Kreise sind dabei gegeben durch $(-6,0),(-3,0),(0,0),(3,0)$ und $(6,0)$. Die Wellenzahlen und Übergangskonstanten wurden genau wie im ersten Beispiel gewählt. Weiterhin betrachten wir Gebiete, die sowohl ineinander-, als auch nebeneinander liegen (vgl. Abbildung 7.5). Der äußere Kreis hat hier den Radius drei, die anderen Ränder werden um den Faktor zwei im Vergleich $\mathrm{zu}$ den in der Bemerkung 7.5 angegebenen Parametrisierungen gestaucht. Als Wellenzahlen wurden $\kappa_{0}=1.9, \kappa_{1}=1.0$ und $\kappa_{2}=\kappa_{3}=\kappa_{4}=\kappa_{5}=0.8$ gewählt. Die Übergangskonstanten $\rho_{j}$ wurden wie bei den ersten beiden Beispielen gewählt.

Als letztes haben wir eine etwas kompliziertere Geometrie untersucht (vgl. Abbildung 7.6). Der Drache wurde hier um 2.5 gestreckt, die Raumschiffe um den Faktor 2 und die Bohne um den Faktor 5 gestaucht. Die Wellenzahlen lauteten: $\kappa_{0}=1.9$, $\kappa_{1}=1.0, \kappa_{2}=\ldots=\kappa_{7}=0.8$ und $\kappa_{8}=1.3$ ( $\Omega_{8}$ ist hierbei die Bohne). Als Übergangskonstanten haben wir gewählt: $\rho_{1}=\rho_{3}=\rho_{5}=0.7, \rho_{2}=\rho_{4}=0.5, \rho_{6}=\rho_{7}=0.6$ und $\rho_{8}=0.9$.

In der Tabelle 7.3 ist folgender Fehler für die drei Beispielgeometrien (mit Geo1, Geo2 und Geo3 bezeichnet) eingetragen: Zunächst wurde der Wert $u_{256, \infty}$ des Fernfeldes für eine einfallende ebene Welle mit Einfallsrichtung $(1,0)$ bei 256 Knotenpunkten (d.h. 256 pro Kreis) bestimmt. In der Tabelle sind dann die Werte $\left|u_{n, \infty}(1,0)-u_{256, \infty}(1,0)\right|$ eingetragen. Bei diesem Beispiel zeigt sich deutlich, daß zur numerischen Lösung von 


\begin{tabular}{lcccccccc}
\hline \hline $\mathrm{n}$ & Geo1 & Zeit & Geo1(komplex) & Zeit & Geo2 & Zeit & Geo3 & Zeit \\
\hline 32 & 0.05 & 0 & 0.1 & 0 & $1.23 \mathrm{e}-11$ & 1 & 0.0007 & 1 \\
64 & $9.3 \mathrm{e}-5$ & 1 & $2.8 \mathrm{e}-5$ & 2 & $1.49 \mathrm{e}-15$ & 6 & $1.9 \mathrm{e}-7$ & 6 \\
128 & $1.22 \mathrm{e}-10$ & 12 & $8.5 \mathrm{e}-8$ & 15 & $2.31 \mathrm{e}-15$ & 47 & $2.7 \mathrm{e}-14$ & 47 \\
256 & - & 109 & - & 119 & - & 401 & - & 407 \\
\hline \hline
\end{tabular}

Tabelle 7.3: Konvergenzgeschindigkeit bei verschiedenen Gebieten mit mehreren Rändern

\begin{tabular}{lcccccc}
\hline \hline $\mathrm{n}$ & Geo4(LU) & Zeit & Geo4(ESV14) & Zeit & Geo4(ESV6) & Zeit \\
\hline 32 & 0.02 & 2 & 0.03 & 3 & 0.03 & 1 \\
64 & 0.003 & 17 & 0.0009 & 18 & 0.0009 & 7 \\
128 & $4.4 \mathrm{e}-5$ & 134 & $2.16 \mathrm{e}-5$ & 72 & $6.1 \mathrm{e}-5$ & 27 \\
256 & - & 1154 & $5.0 \mathrm{e}-8$ & 296 & $4.1 \mathrm{e}-5$ & 115 \\
\hline \hline
\end{tabular}

Tabelle 7.4: Konvergenzgeschindigkeit bei Geometrie 4 mit LU-Zerlegung und Einzelschrittverfahren.

ineinanderliegenden Gebieten mehr Knotenpunkte erforderlich sind, um die gleiche Genauigkeit wie bei nebeneinanderliegenden Gebieten zu erhalten. Dieses Verhalten ist zwar zu einem gewissen Grad abhängig von den Wellenzahlen, ist aber bei einer Reihe von Versuchen aufgetreten. Der Aufwand bei der Verwendung von komplexen Wellenzahlen ist kaum größer als im reelen Fall. Auch die Approximationsgüte ist vergleichbar.

In der Tabelle 7.4 haben wir die gleichen Werte für die Geometrie 7.6 bestimmt. Die Berechnung für 256 Knotenpunkte erforderte fast 20 Minuten (zu Lösen war ein komplexes, fast vollbesetztes, $4096 \times 4096$ Gleichungssystem - die Blockmatrix hat Kantenlänge 8 und jeder Block hat Kantenlänge $2 \times 256$; der Speicherbedarf betrug 410MByte). Hier scheint es für zukünftige Versuche ratsam zu sein, iterative Verfahren (z.B. Zweischritt- bzw. Multgridverfahren) zu verwenden (vgl. hierfür z.B. [37], Kapitel 14.2 und 14.3). Exemplarisch betrachten wir ein relaxiertes (Parameter 0.5) Einzelschrittverfahren für den Greenschen Ansatz. In der Tabelle 7.4 wurde wiederum die Differenz zu der durch die LU-Zerlegung ermittelten Lösung angegeben. Bei iterativen Verfahren kann durch ein geeignetes Abbruchkriterium die Genauigkeit der Näherungslösung für ein Gleichungssystem bestimmt werden. Dies gibt eine weitere Möglichkeit zur Beschleunigung des Verfahrens. In der Tabelle 7.4 haben wir zwei Varianten (mit ESV14 und ESV6 bezeichnet) dazu angegeben. Einmal wurde die Iteration abgebrochen, wenn sich die Norm zweier aufeinanderfolgender Iterationsvektoren nur noch um $10^{-14}$ unterschied, beim anderen Versuch bei $10^{-6}$. Gerade unter dem Gesichtspunkt, daß Meßdaten zumeist fehlerbehaftet sind und es daher keinen Sinn macht, die Daten zu genau zu approximieren, ist dies eine sinnvolle Möglichkeit zur 
Geschwindigkeitssteigerung des Verfahrens. Da wir keine Aussagen über Konvergenz des Einzelschrittverfahrens treffen können, handelt es sich hier um ein rein experimentelles Ergebnis. Der Zeitunterschied (115 bzw. 296 statt 1154 Sekunden Laufzeit bei 256 Knotenpunkten) zeigt aber deutlich, daß es sinnvoll ist, bei Problemen dieser Größe iterative Verfahren einzusetzen.

\subsection{Berechnung der Fréchet-Ableitungen}

In diesem Teil des Kapitels wollen wir Verfahren zur Berechnung der FréchetAbleitungen angeben. Auch wenn wir die Existenz von höheren Ableitungen in Kapitel 6 nachgewiesen hatten, werden wir bei den praktischen Beispielen nur die erste Ableitung verwenden. Die effektivste Methode zur Berechnung der Ableitung nach dem Rand ist die Darstellung als Randwertproblem. Wir betrachten zunächst die Situation bei einem zusammenhängenden Rand $\Gamma$ mit Außenraum $\Omega_{0}$ und Innenraum $\Omega_{1}$. Es sei $\left(u_{0}, u_{1}\right)$ die Lösung des Streuproblems und $u:=u_{0}+u_{i}$ das gesamte äußere Feld. Weiterhin sei $z \in C^{2, \alpha}\left([0,2 \pi], \mathbb{R}^{2}\right)$ eine Parametrisierung des Randes und $h \in C^{2, \alpha}\left([0,2 \pi], \mathbb{R}^{2}\right)$ eine Störung. Wie in Kapitel 6 gezeigt, kann die Ableitung $u_{\infty}^{\prime}[z ; h]$ durch folgendes Randwertproblem bestimmt werden:

Problem 7.6. Gesucht sind $v_{j} \in C^{2}(\Omega) \cap C^{1}(\bar{\Omega}), j=0,1$ mit

$$
\left.\left(\Delta+\kappa_{j}^{2}\right) v_{j}\right|_{\Omega_{j}}=0, \quad j=0,1 .
$$

Weiterhin erfülle $v_{0}$ die Sommerfeldsche Ausstrahlungsbedingung (das Fernfeld von $v_{0}$ bezeichnen wir mit $v_{\infty}$ ) und die Felder $v_{0}$ und $v_{1}$ haben die Randwerte

$$
\begin{aligned}
\left.v_{1}\right|_{\Gamma}-\left.v_{0}\right|_{\Gamma} & =\left.\nabla u \cdot h\right|_{\Gamma}-\left.\nabla u_{1} \cdot h\right|_{\Gamma} \\
\frac{\partial v_{1}}{\partial \nu}-\rho \frac{\partial v_{0}}{\partial \nu} & =\left(\rho \nabla u-\nabla u_{1}\right) \cdot \nu^{\prime}[z ; h]+\left(\rho \nabla^{2} u_{0}-\nabla^{2} u_{1}\right) \cdot h \nu .
\end{aligned}
$$

Es hatte sich gezeigt, daß die Gleichung $v_{\infty}=u_{\infty}^{\prime}[z ; h]$ gilt. Somit kann also die Ableitung von $u_{\infty}$ in Richtung $h$ durch dieses Randwertproblem bestimmt werden. Zur praktischen Berechnung ist es jedoch günstiger, die Randwerte in einer anderen Form zu schreiben. Für die Dirichlet-Werte folgt aus $u=u_{1}$, daß die Tangentialkomponente dieser Differenz verschwindet. Somit gilt die Gleichung

$$
\left.v_{1}\right|_{\Gamma}-\left.v_{0}\right|_{\Gamma}=h \cdot \nu\left(\frac{\partial u}{\partial \nu}-\frac{\partial u_{1}}{\partial \nu}\right)
$$

Bei den Neumann-Randwerten kann durch differentialgeometrische Überlegungen (vgl. [24], Lemma 4.5, bzw. [27], Proposition 1.24; eine andere Herleitung findet man in [39]) die folgende Gleichung bewiesen werden:

$$
\frac{\partial v_{1}}{\partial \nu}-\rho \frac{\partial v_{0}}{\partial \nu}=\frac{\mathrm{d}}{\mathrm{ds}}\left(h \cdot \nu \frac{\mathrm{d}}{\mathrm{ds}}\left(u_{1}-\rho u\right)\right)+h \cdot \nu\left(\kappa_{1}^{2} u_{1}-\rho \kappa_{0}^{2} u\right)
$$




\begin{tabular}{lcrcc}
\hline \hline $\mathrm{N}$ & Potential & Zeit & Green & Zeit \\
\hline 32 & 0.31630040 & 0 & 0.31638891 & 0 \\
64 & 0.31481879 & 0 & 0.31481879 & 0 \\
128 & 0.31482369 & 0 & 0.31482369 & 0 \\
256 & 0.31482369 & 3 & 0.31482369 & 3 \\
\hline \hline
\end{tabular}

Tabelle 7.5: Vergleich von Potential- und Greenschem Ansatz bei der Ableitung nach dem Rand

Der entscheidende Punkt bei diesen beiden Gleichungen für die Randwerte liegt darin, daß zu ihrer Berechnung nur die Cauchy-Daten von $u$ benötigt werden (die CauchyDaten von $u_{1}$ ergeben sich dann aus der Randbedingung für das direkte Problem). Somit erhalten wir folgendes Verfahren zur Berechnung der Fréchet-Ableitung nach dem Rand für den Potentialansatz:

1. Bestimme eine Approximation $u_{c, n}$ an die Cauchy-Daten $u_{c}$ von $u_{0}$ in der Form eines trigonometrischen Polynoms durch Lösen der Gleichung (7.1) und Einsetzen der Lösung in (7.2).

2. Berechne hiermit eine Approximation an die erste und zweite Ableitung der Dirichlet-Randwerte von $u$ durch Differenzieren des trigonometrischen Polynoms $u_{c, n, 1}$.

3. Bestimme hieraus eine Approximation an die Randwerte von $\left(v_{0}, v_{1}\right)$ und damit eine Approximation an $u_{\infty}^{\prime}[z ; h]$. Hierbei kann die LU-Zerlegung, die im ersten Schritt berechnet wurde, wieder verwendet werden.

Hier zeigt sich ein Vorteil des Greenschen Ansatzes: Die beim Lösen des direkten Problems bestimmte Dichte $\varphi$ enthält gerade die Cauchy-Daten der äußeren Felder, so daß hier im Gegensatz zum Potentialansatz keine weiteren Umwandlungen vorgenommen werden müssen. Allerdings wird dafür beim Lösen des direkten Problems eine weitere Matrix-Vektor-Multiplikation zur Bestimmung der rechten Seite benötigt. Weiterhin beachte man, daß wir für das eben verwendete Verfahren ausgenutzt haben, daß die Cauchy-Daten durch ein trigonometrisches Polynom approximiert wurden und daher einfach differenziert werden können. In der Tabelle 7.5 haben wir Potential- und Greenschen Ansatz verglichen. Als Rand wurde der Drache gewählt, die Störung $h$ war gegeben durch

$$
h(t):=\cos 2 t\left(\begin{array}{c}
\cos t \\
\sin t
\end{array}\right) .
$$

Der dargestellte Funktionswert ist $\operatorname{Re}\left(u_{\infty}^{\prime}[z ; h](1,0)\right)$. Die Unterschiede zwischen Potential- und Greenschem Ansatz sind marginal. Der Grund, mehr als einen Ansatz zu implementieren, waren auch keine Effizienzerwägungen, sondern die Möglichkeit, durch Vergleich von Ergebnissen, Fehler im Programm finden zu können, bzw. die Korrektheit des Programms sicherstellen zu können. 


\begin{tabular}{ccc}
\hline \hline Iteration & $\left|\kappa_{i}-\kappa_{i}^{\text {approx }}\right|$ & $\left|\rho-\rho^{\text {approx }}\right|$ \\
\hline 1 & 0.03 & 0.03 \\
2 & $3 \mathrm{e}-4$ & $6 \mathrm{e}-4$ \\
3 & $6 \mathrm{e}-8$ & $3 \mathrm{e}-7$ \\
4 & $2 \mathrm{e}-13$ & $2 \mathrm{e}-12$ \\
5 & $7 \mathrm{e}-16$ & $3 \mathrm{e}-16$ \\
\hline \hline
\end{tabular}

Tabelle 7.6: Konvergenzgeschindigkeit bei der Rekonstruktion von $\kappa_{i}$ und $\rho$ beim Drachen. 128 Knotenpunkte bei der Berechnung der Meßdaten, wie auch bei der Rekonstruktion, Zeitbedarf: 1 Sekunde insgesamt (d.h. mit Berechnung der Meßdaten). Startwert bei $\kappa_{i}: 0.8$, bei $\rho: 0.9$. Ausgegeben wird die Differenz zwischen eigentlichem Wert und Näherungswert.

Die Berechnung der Ableitung nach der Übergangskonstanten $\rho$ erfolgt analog zu der Berechnung der Ableitung nach dem Rand. In Kapitel 6 wurde gezeigt, daß die Ableitung durch folgendes Problem berechnet werden kann:

Problem 7.7. Gesucht sind $v_{j} \in C^{2}(\Omega) \cap C^{1}(\bar{\Omega}), j=0,1$ mit

$$
\left.\left(\Delta+\kappa_{j}^{2}\right) v_{j}\right|_{\Omega_{j}}=0, \quad j=0,1 .
$$

Weiterhin erfülle $v_{0}$ die Sommerfeldsche Ausstrahlungsbedingung und die Felder $v_{0}$ und $v_{1}$ haben die Randwerte

$$
\begin{gathered}
\left.v_{1}\right|_{\Gamma}-\left.v_{0}\right|_{\Gamma}=0 \\
\frac{\partial v_{1}}{\partial \nu}-\rho \frac{\partial v_{0}}{\partial \nu}=\frac{\partial u}{\partial \nu} .
\end{gathered}
$$

Somit benötigt man zur Berechnung dieser Ableitung nur die Normalableitung der Lösung des direkten Problems.

In beiden Fällen können wir bei mehreren Rändern genauso vorgehen, wie bei einem Rand, d.h. die Ableitungen nach dem Rand bzw. den Übergangskonstanten ergeben sich aus direkten Transmissionsproblemen mit geeigneten Randwerten.

Wie bereits in Kapitel 6 dargestellt wurde, ist die Berechnung der Ableitung nach der Wellenzahl etwas aufwendiger. Hier lösen die differenzierten Funktionen eine inhomogene Helmholtzgleichung, die numerisch aufwendiger zu lösen ist. Um dies zu umgehen, haben wir folgenden Ansatz verwendet: Das Fernfeld der Lösung des direkten Problems ist von der Form

$$
u_{\infty}=F_{\infty} M^{-1} I_{\rho} f
$$

Der Fernfeldoperator $F_{\infty}:(C[0,2 \pi])^{2} \rightarrow L^{2}\left(S^{1}\right)$ ist definiert durch

$$
F_{\infty} \varphi(\hat{x})=\frac{e^{i \pi / 4}}{\sqrt{8 \pi \kappa_{0}}} \int_{0}^{2 \pi} e^{i \kappa_{0} \hat{x} \cdot z(\tau)}\left(-i \kappa_{0} \hat{x} \nu(\tau) \varphi_{1}(\tau)+\varphi_{2}(\tau)\right)\left|z^{\prime}(\tau)\right| \mathrm{d} \tau .
$$




\begin{tabular}{ccc}
\hline \hline Iteration & $\left\|\kappa-\kappa^{\text {approx }}\right\|$ & $\left\|\rho-\rho^{\text {approx }}\right\|$ \\
\hline 1 & 0.12 & 0.04 \\
2 & 0.01 & $2 \mathrm{e}-3$ \\
3 & $9 \mathrm{e}-5$ & $5 \mathrm{e}-6$ \\
4 & $2 \mathrm{e}-8$ & $2 \mathrm{e}-7$ \\
5 & $2 \mathrm{e}-12$ & $2 \mathrm{e}-7$ \\
\hline \hline
\end{tabular}

Tabelle 7.7: Konvergenzgeschindigkeit bei der Rekonstruktion von $\kappa$ und $\rho$ bei der Geometrie 3. 64 Knotenpunkte bei der Berechnung der Meßdaten, wie auch bei der Rekonstruktion. Zeitbedarf: 34 Sekunde insgesamt (d.h. mit Berechnung der Meßdaten). Startwert bei $k: 0.9$, bei $\rho: 0.6$. Ausgegeben wird die Norm der Differenz zwischen eigentlichem Wert und Näherungswert.

Da beim Potentialansatz nur der Operator $M$ von der inneren Wellenzahl abhängt, ergibt sich

$$
u_{\infty}^{\prime}\left[\kappa_{1}\right]=-F_{\infty} M^{-1} M^{\prime}\left[\kappa_{1}\right] M^{-1} I_{\rho} f .
$$

Notwendig ist also die Berechnung von $M^{\prime}\left[\kappa_{1}\right]$. Hierzu gehen wir genau wie beim direkten Problem vor, d.h. wir spalten die auftretenden logarithmischen Singularitäten der Kerne ab und integrieren unter Verwendung von trigonometrischer Interpolation. Auch hier ist die Übertragung auf die Situation bei mehreren Randkurven direkt möglich.

\subsection{Inverse Transmissionsprobleme}

Im letzten Abschnitt wollen wir uns nun mit einigen numerischen Aspekten bei der Rekonstruktion von Rand, Wellenzahlen und Übergangskonstanten beim Transmissionsproblem beschäftigen. Sei hierzu

$$
\begin{aligned}
\mathcal{Z}:= & \left\{z \in C^{2, \alpha}\left(\mathbb{R}, \mathbb{R}^{2}\right): z \text { ist } 2 \pi \text {-periodisch, injektiv in }[0,2 \pi[\right. \\
& \text { und } \left.\left|z^{\prime}(t)\right|>0 \text { für alle } t \in \mathbb{R} .\right\}
\end{aligned}
$$

eine Menge von regulären Parametrisierungen und $\mathcal{W} \subset \mathbb{C}^{L}$ und $\mathcal{U} \subset \mathbb{C}^{L}$ seien die Mengen der inneren Wellenzahlen und der Übergangskonstanten. Die äußere Wellenzahl $\kappa_{0}$ halten wir fest. Dies ist auch aus praktischen Gesichtspunkten vernünftig, da im äußere Gebiet die Meßdaten erhoben werden, d.h. die Wellenzahl $\kappa_{0}$ ist bei der Erhebung der Meßdaten bereits bekannt. Wir setzen voraus, daß für alle $(\kappa, \rho) \in \mathcal{W} \times \mathcal{U}$ das direkte Transmissionsproblem eindeutig lösbar ist.

Sei nun für $(z, \kappa, \rho) \in \mathcal{Z} \times \mathcal{W} \times \mathcal{U}$ die Funktion $u_{\infty}$ das Fernfeld beim Transmissionsproblem für die Parameter $(z, \kappa, \rho)$ und fester Einfallswelle $u_{i}$, d.h. in Operatornotation gilt

$$
F[z, \kappa, \rho]=u_{\infty}
$$


mit einem nichtlinearen Operator $F$. Diese Gleichung versuchen wir nun durch ein Newton-Verfahren zu lösen, d.h. wir betrachten stattdessen die linearisierte Gleichung

$$
\frac{\partial}{\partial z} F\left[z, \kappa, \rho ; h_{z}\right]+\frac{\partial}{\partial \kappa} F\left[z, \kappa, \rho ; h_{\kappa}\right]+\frac{\partial}{\partial \rho} F\left[z, \kappa, \rho ; h_{\rho}\right]+F[z, \kappa, \rho]=u_{\infty} .
$$

Ausgehend von einer Startnäherung $\left(z_{0}, \kappa_{0}, \rho_{0}\right)$ bestimmt man also eine Lösung $\left(h_{z}, h_{\kappa}, h_{\rho}\right)$ und damit eine neue Näherung $\left(z_{1}, \kappa_{1}, \rho_{1}\right)=\left(z_{0}, \kappa_{0}, \rho_{0}\right)+\left(h_{z}, h_{\kappa}, h_{\rho}\right)$. Dieses Verfahren wird dann iterativ fortgeführt.

Hierbei ist zu beachten, daß die Gleichung (7.10) schlecht-gestellt ist und dies vererbt sich auch auf die Gleichung (7.11) (vgl. hierzu [57], Satz 5.9). Daher betrachten wir stattdessen das regularisierte Minimierungsproblem

$$
\begin{aligned}
\left\|\frac{\partial}{\partial z} F\left[z, \kappa, \rho ; h_{z}\right]+\frac{\partial}{\partial \kappa} F\left[z, \kappa, \rho ; h_{\kappa}\right]+\frac{\partial}{\partial \rho} F\left[z, \kappa, \rho ; h_{\rho}\right]+F[z, \kappa, \rho]-u_{\infty}\right\|_{L^{2}}^{2} \\
+\mu_{z}\left\|h_{z}\right\|_{3}^{2}+\mu_{\kappa}\left|h_{\kappa}\right|+\mu_{\rho}\left|h_{\rho}\right|=\min !
\end{aligned}
$$

mit Regularisierungsparametern $\mu_{z}, \mu_{\kappa}$ und $\mu_{\rho}$. Um nun zu einem endlichdimensionalen Problem zu gelangen, approximieren wir die Normen $\|\cdot\|_{L^{2}}$ und $\|\cdot\|_{3}$ durch

$$
\begin{aligned}
\|f\|_{L^{2}, N}^{2} & :=\frac{1}{N} \sum_{n=0}^{N-1}\left|f\left(\frac{2 \pi n}{N}\right)\right|^{2} \quad N \in \mathbb{N}, \\
\|f\|_{3, K}^{2} & :=\frac{1}{K} \sum_{k=0}^{K-1}\left(\left|f\left(\frac{2 \pi k}{K}\right)\right|^{2}+\left|f^{(3)}\left(\frac{2 \pi k}{K}\right)\right|^{2}\right) \quad K \in \mathbb{N} .
\end{aligned}
$$

Weiterhin wählen wir die Störung $h_{z}$ des Randes aus einem endlichdimensionalen Raum. In dieser Arbeit verwenden wir zwei Varianten. Für sternförmige Gebiete wählen wir Abbildungen der Form

$$
h_{z}(t)=p(t)\left(\begin{array}{c}
\cos t \\
\sin t
\end{array}\right)
$$

Als zweite Variante, die uns auch die Behandlung von nichtsternförmigen Gebieten ermöglicht, verwenden wir Funktionen der Form

$$
h_{z}(t)=\left(\begin{array}{l}
p_{1}(t) \\
p_{2}(t)
\end{array}\right)
$$

In beiden Fällen wählen wir die Funktionen $p, p_{1}, p_{2}$ aus einem endlichdimensionalen Unterraum der trigonometrischen Polynome.

Insgesamt erhalten wir somit ein Problem der kleinsten Quadrate (vgl. z.B. [28], Kapitel 8 für eine genauere Darstellung).

Für die theoretischen Aspekte bei Newtonverfahren dieser Art verweisen wir auf Hohage [27] (vgl. auch. [25], [26]). In dieser Arbeit werden Aussagen bzgl. Konvergenz und Konvergenzgeschwindigkeit bei iterativen Verfahren zur Lösung von inversen 

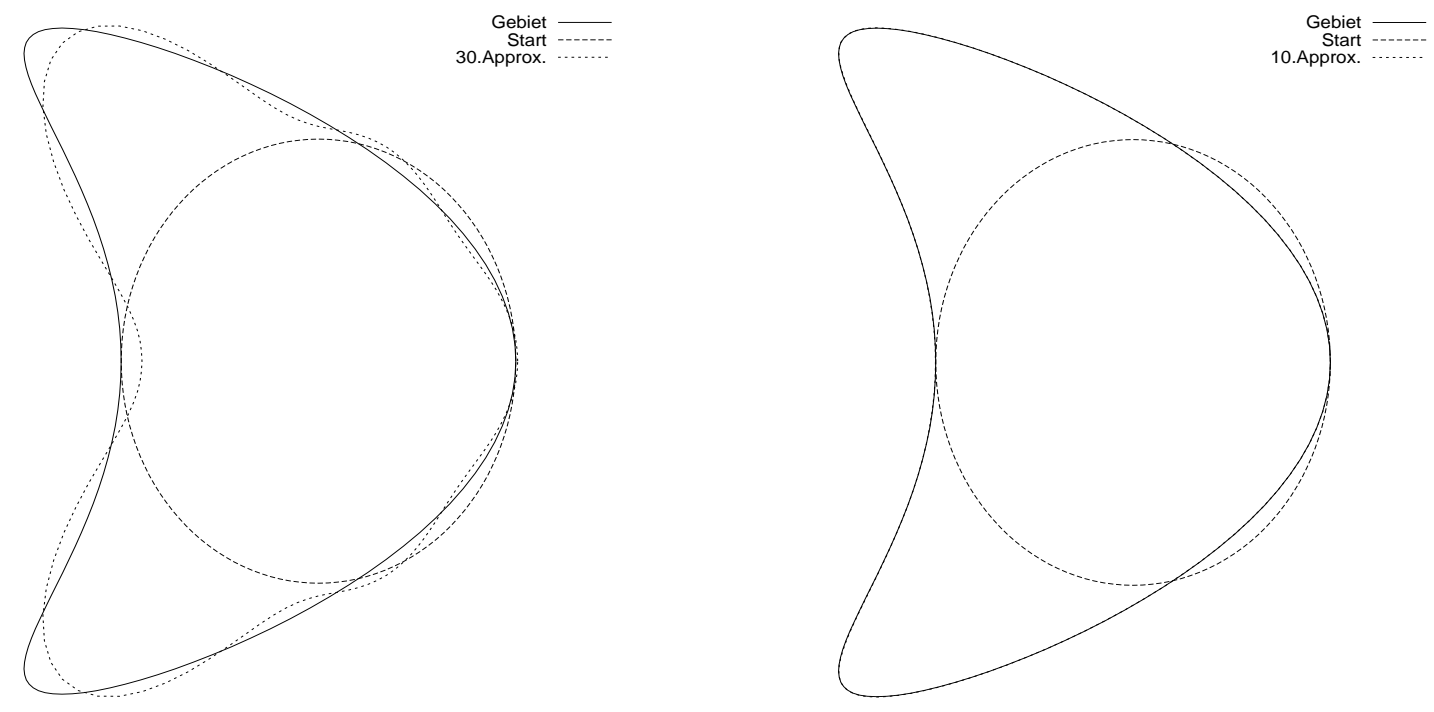

Abbildung 7.7: Rekonstruktion des Drachens bei einer Einfallswelle; links wird sternförmig mit 30 Basisfunktionen approximiert; rechts wird nichtradial approximiert; hier wurden 10 Basisfunktionen verwendet.
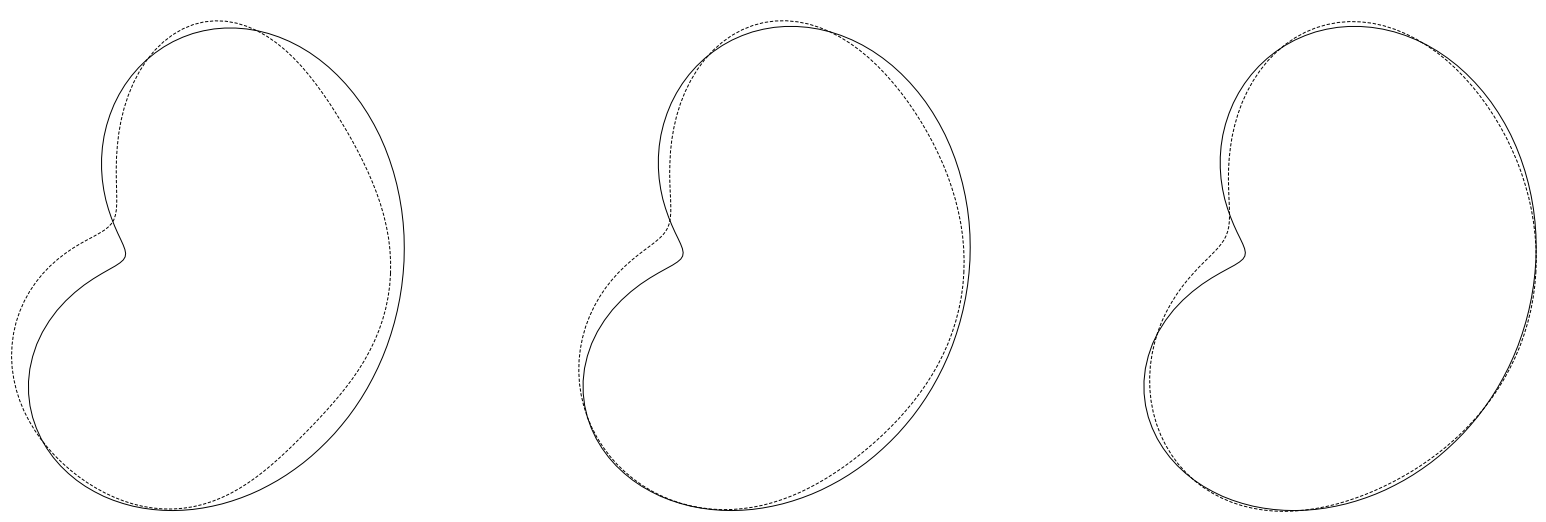

Abbildung 7.8: Rekonstruktion der Bohne mit 10\%, 5\% und 1\% Datenfehler; 10 radiale Ansatzfunktionen; 20 Iterationen; 2 Sekunden Gesamtlaufzeit (inklusive Berechnung der Daten); 

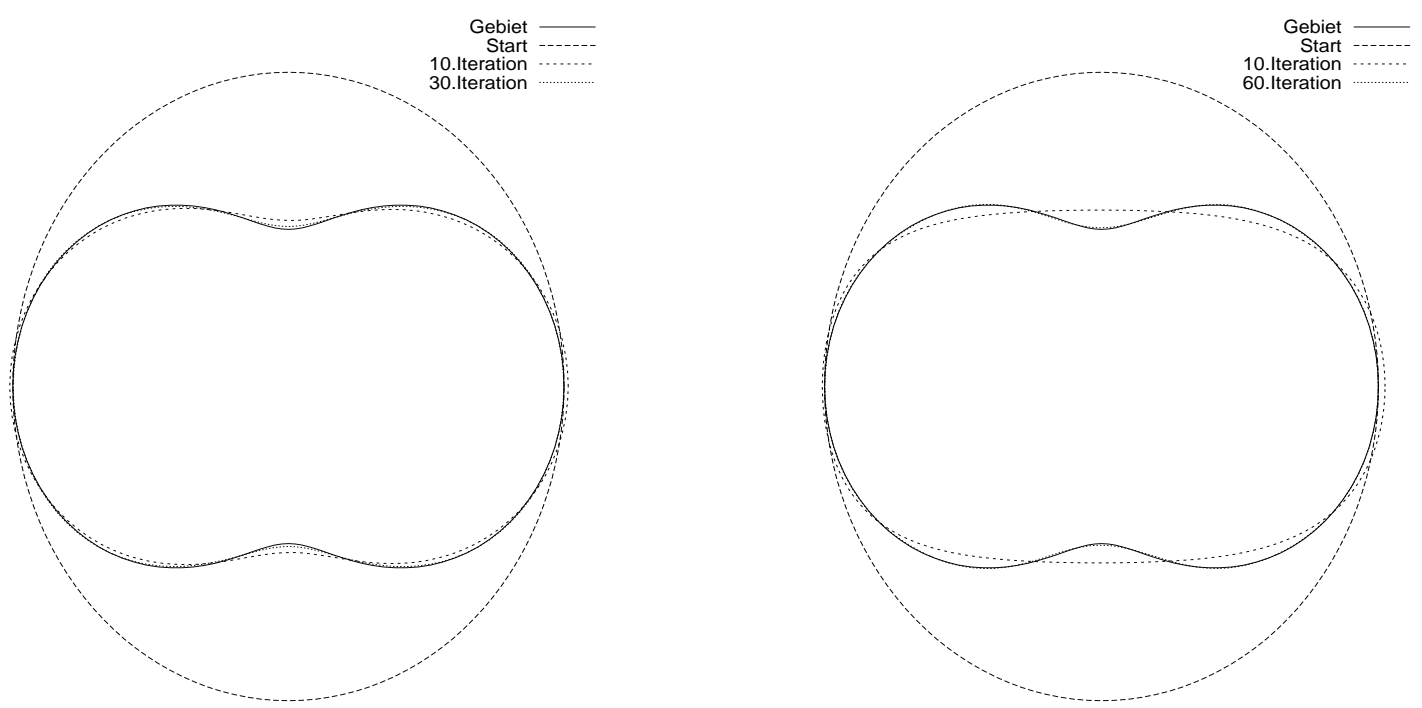

Abbildung 7.9: Rekonstruktion der Erdnuß bei einer Einfallswelle; links wird sternförmig mit 30 Basisfunktionen approximiert; rechts wird nichtsternförmig approximiert; hier wurden ebenfalls 30 Basisfunktionen verwendet.

Problemen bewiesen. Allerdings konnten bisher bei inversen Streuproblemen für die Helmholtzgleichung nicht alle benötigten Voraussetzungen nachgewiesen werden.

Nun betrachten wir einige numerische Beispiele. Zunächst wollen wir bei festem Rand die innere(n) Wellenzahl(en), bzw. die Übergangskonstante(n) rekonstruieren. Wie in Tabelle 7.6 und Tabelle $7.7 \mathrm{zu}$ sehen ist, funktioniert dies bei festem Rand sehr gut.

Jetzt wenden wir uns der Rekonstruktion des Randes bei fester Wellenzahl und fester Übergangskonstante zu.

Bemerkung 7.8. Falls nichts anderes angegeben ist, haben wir bei der Rekonstruktion eines Randes $\kappa_{0}=1.9$ und $\kappa_{1}=1.0$ gewählt. Auf dem Kreis und auf dem Rand (bzw. den Rändern der Gebiete) wurden 64 Knotenpunkte verwendet. Die Norm $\|\cdot\|_{3, K}^{2}$ wurde normalerweise für $K=128$ berechnet. Bei komplizierteren Objekten hat es sich als notwendig erwiesen, mehrere Einfallswellen zu verwenden. Die Einfallsrichtungen wurden dann gleichmäßig auf dem Einheitskreis verteilt. Bei einer Einfallsrichtung war diese stets durch $v=(1,0)$ gegeben. Der Regularisierungsparameter $\alpha$ hatte normalerweise den Startwert $\alpha=0.0005$ und wurde in jedem Iterationsschritt mit 0.9 multipliziert. Als Startkurve des Iterationsverfahrens haben wir stets den Einheitskreis (bzw. mehrere Einheitskreise) verwendet. Wir haben die Iteration nach einer Anzahl von Schritten, die wir jeweils angegeben haben, abgebrochen. In unseren Beispielen wollen wir nur die grundsätzliche Durchführbarkeit des Newtonverfahrens demonstrieren. Für verschiedene a-priori bzw. a-posteriori Abbruchkriterien bei regularisierten Newtonverfahren verweisen wir auf die Dissertation von Hohage [27]. 


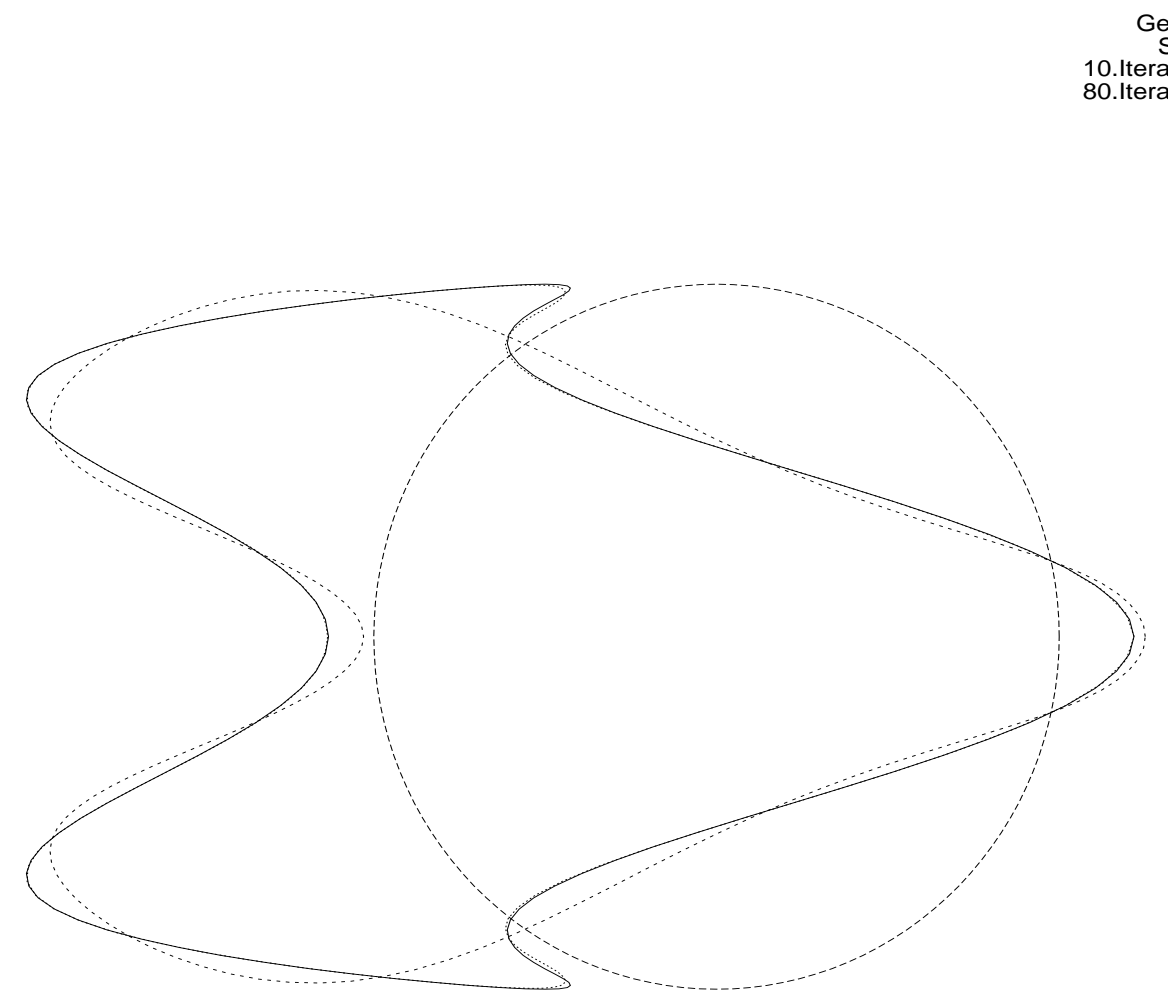

Abbildung 7.10: Rekonstruktion des Raumschiffs bei acht Einfallswellen; hier wird nur nichtsternförmig approximiert, da die sternförmige Variante scheitert. Die Meßdaten wurden mit 256 Knotenpunkten erzeugt, approximiert wird mit 128 Knotenpunkten; die Laufzeit betrug 4m20sec.

In der Abbildung 7.7 betrachten wir die Rekonstruktion des Drachens. Dieses Gebiet wurde in der Vergangenheit häufig als Testbeispiel für Rekonstruktionsverfahren benutzt und hat schon fast eine Art Benchmark-Status erlangt. In der Abbildung 7.7 wurden links 30 radiale Basisfunktionen verwendet. Nach 30 Schritten ist die Approximation recht gut, die Bereiche mit der stärksten Krümmung werden allerdings nicht genau rekonstruiert. Bei der nichtradialen Approximation ist die Rekonstruktion des Drachens sehr einfach. Der Grund hierfür ist die Tatsache, daß die Parametrisierung im Ansatzraum enthalten ist. Daher ist dies bei nichtradialer Ansatzfunktion auch kein geeignetes Testgebiet.

In den Abbildungen 7.8 wurde die Bohne rekonstruiert. Hierbei haben wir die Fernfelddaten mit einem Fehler addiert. Die Versuche zeigen, daß sich das Verfahren stabil gegenüber Datenfehlern verhält. In der Abbildung 7.9 wurde die Erdnuß mit radialen und nichtradialen Basisfunktionen rekonstruiert. Interessanterweise liefert hier die radialen Ansatzfunktionen die besseren Ergebnisse. Erst die Abbildung 7.10 zeigt die Vorteile der nichtradialen Basisfunktionen. Da das Raumschiff nicht sternförmig ist, versagt hier der radiale Ansatz, während durch nichtradiale Approximation des Gebiet sehr gut rekonstruiert werden kann. Man beachte allerdings, daß acht Einfallswellen 


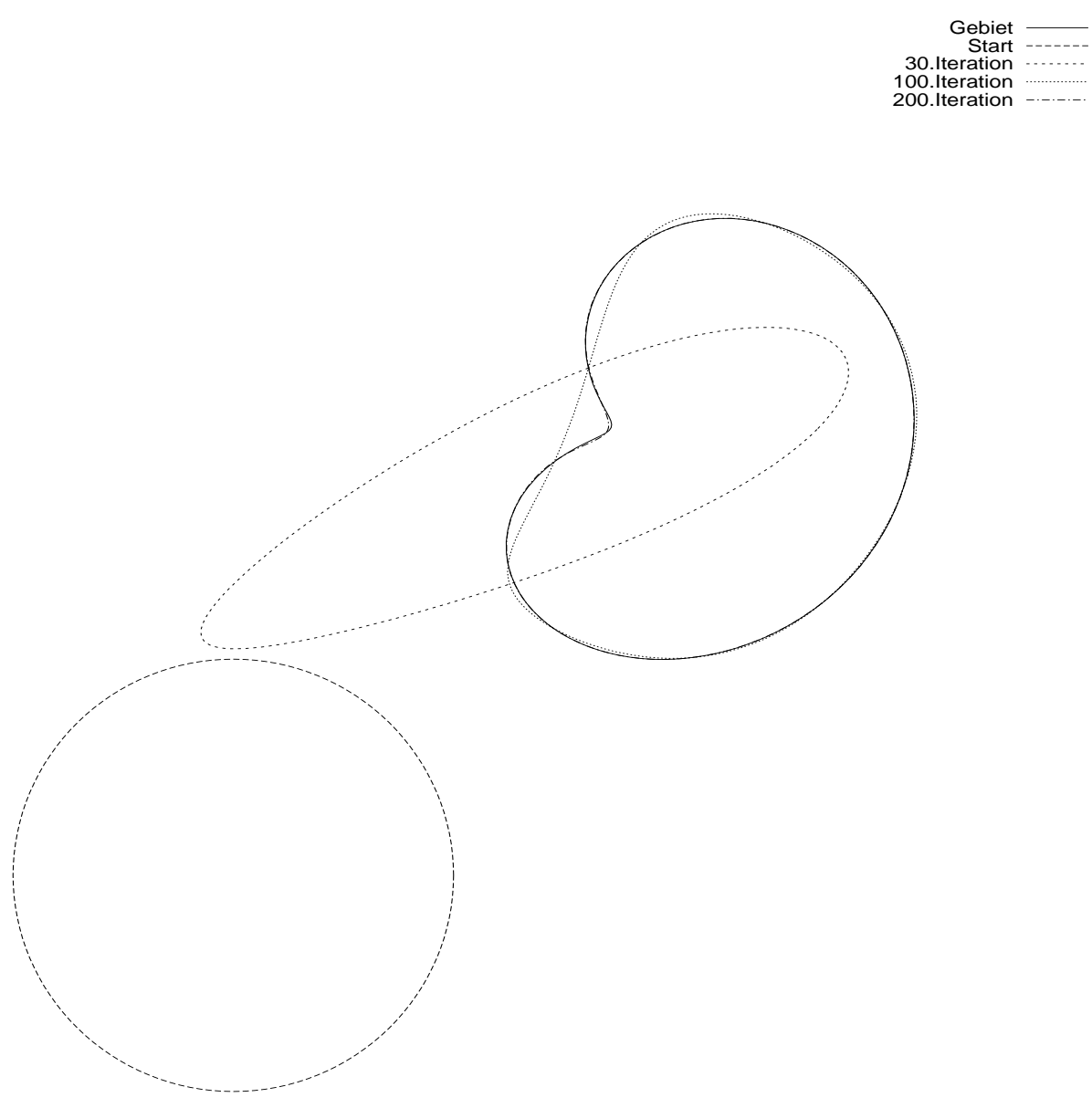

Abbildung 7.11: Rekonstruktion der Bohne bei einer Einfallswelle; hier wird nur nichtsternförmig approximiert; entscheidend ist hier ein großer Regularisierungsparameter $(\alpha=5$.) zu Beginn des Verfahrens. Laufzeit: 34 Sekunden

verwendet wurden und zur Gewinnung der synthetischen Daten 256 und bei der Rekonstruktion 128 Knotenpunkte verwendet wurden. Hier scheint sich abzuzeichnen, daß für kompliziertere Geometrien ein erheblich höherer Aufwand entsteht. Als nächstes wollen wir zeigen, daß mit der nichtsternförmigen Approximation auch die Lage eines Objektes bestimmt werden kann. Dies ist der zweite große Vorteil bei dieser Wahl der Ansatzfunktionen. In der Abbildung 7.11 wurde die Bohne rekonstruiert. Ausgangskurve war wie immer ein Kreis, wobei die Bohne allerdings um den Vektor $(3,3)$ verschoben wurde. Man beachte, daß als Startwert für den Regularisierungsparameter in diesem Versuch $\alpha=5.0$ gewählt wurde. Dadurch wurden höherfrequente Anteile bei den Ansatzfunktionen zu Beginn des Iterationsverfahrens sehr stark bestraft. Alternativ könnte man hier auch mit einem zwei-, bzw. mehrstufigen Verfahren arbeiten, bei dem in einem ersten Schritt die Lage des Gebietes mit nur zwei Ansatzfunktionen (d.h. gerade den Basisvektoren im $\mathbb{R}^{2}$ ) bestimmt wird, und erst danach durch Basisfunktionen höherer 

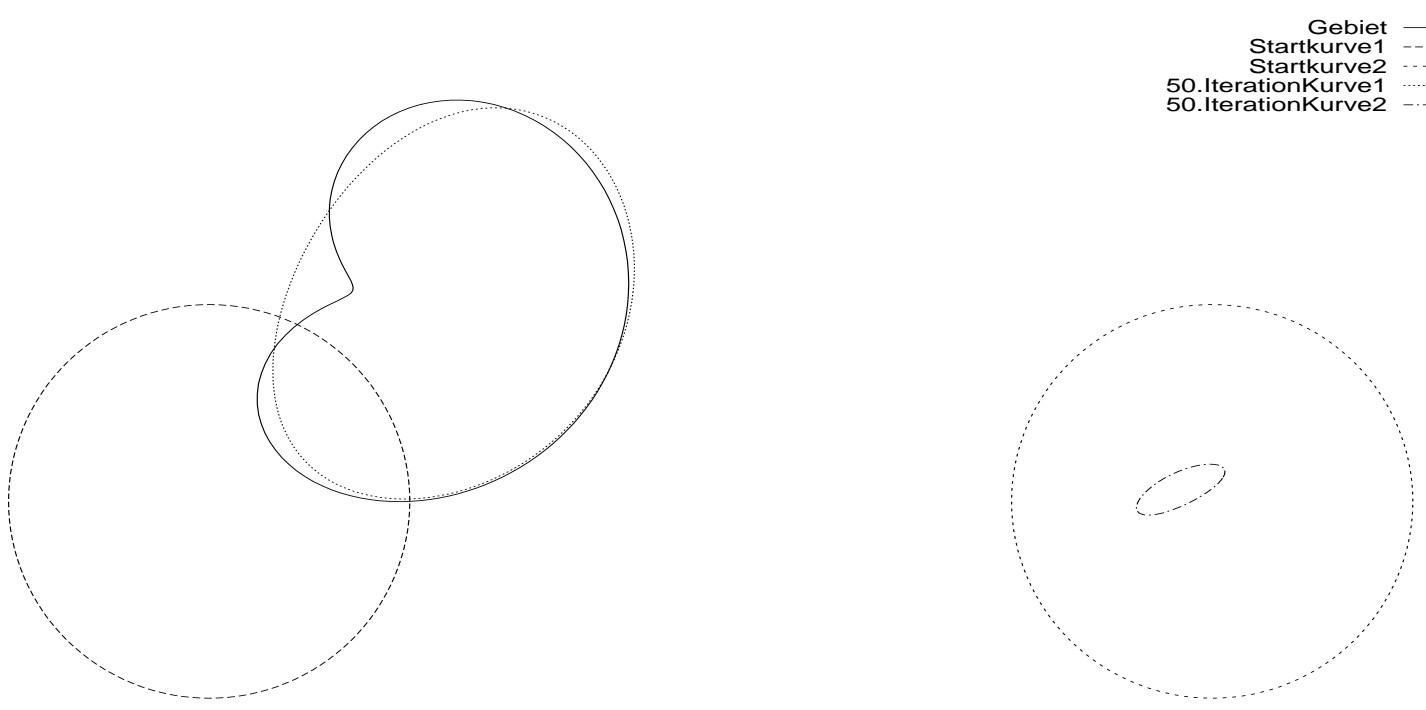

Abbildung 7.12: Rekonstruktion der Bohne bei einer Einfallswelle; 64 Knotenpunkte auf den Approximationskurven; 30 Basisfunktionen; Laufzeit: 55 Sekunden,

Ordnung die Form des Gebietes ermittelt wird. Diese Vorgehensweise wurde in [57], Kapitel 6.6, auch bereits mit Erfolg implementiert. Ein Problem bei dem hier aufgezeigten Verfahren ist, daß die Anzahl der gesuchten Gebiete a-priori bekannt sein muß. In nächsten Versuch (Abbildung 7.12) wird eine Ausgangskurve durch zwei Approximationskurven gesucht. Es zeigt sich, daß die dem Objekt näherliegende Startkurve eine recht gute Approximation des Gebiets liefert, während die zweite Kurve immer kleiner wird. Allerdings lassen sich dadurch ohne irgendeine Form von a-priori Wissen über Lage, Zahl, oder Größe der betrachteten Gebiete auch keine weiteren Schlüsse ziehen. In dieser Situation sind iterative Verfahren, die stets eine hinreichend gute Startnäherung voraussetzen, wahrscheinlich nicht die geeigneten Methoden. Hier könnte sich der Ansatz von Colton und Kirsch (vgl. [5]) als geeignet erweisen. Auch von Potthast (vgl. [50], [52], [53]) wurden diesbzgl. Untersuchungen durchgeführt.

In der Abbildung 7.13 wurden alle Parameter (d.h. Rand, innere Wellenzahl und Übergangskonstante) rekonstruiert. Dies erweist sich als erheblich schwieriger als bei bekannten Konstanten. Um ein Oszillieren der Konstanten während der Iteration zu vermeiden, wurde hier ein größerer Regularisierungsparamter gewählt. Möglichweise sollte man statt gleichzeitiger Rekonstruktion aller Parameter ein mehrstufiges Verfahren verwenden, wobei in jeder Stufe des Verfahrens nur ein Parameter rekonstruiert wird und die anderen festgehalten werden.

Nun noch die Approximations von Gebieten mit Ecken. Wie wir bereits erwähnt haben, sind diese Ergebnisse rein experimenteller Natur, da wir die Ableitung nach dem Rand nur für mindestens $C^{2, \alpha}$-glatte Ränder nachgewiesen haben. Die Versuche scheinen jedoch zumindest darauf hinzuweisen, daß Newtonverfahren beim Transmissionsproblem auch für Gebiete mit Ecken verwendet werden können. Wie bereits erwähnt 

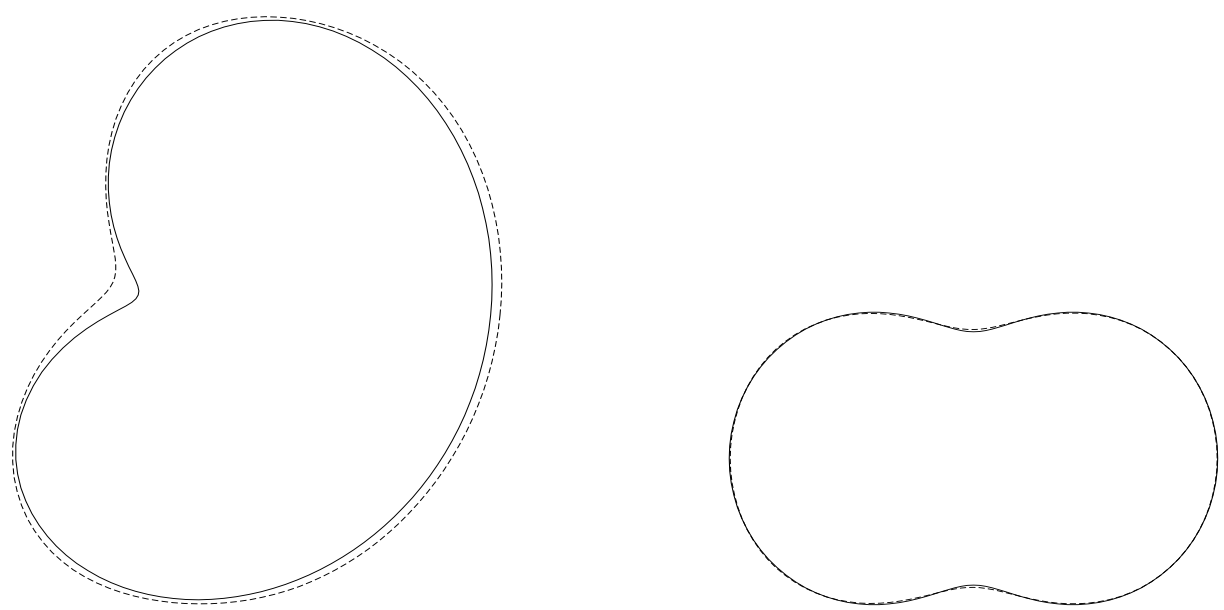

Abbildung 7.13: Rekonstruktion von Bohne und Erdnuß und der inneren Wellenzahl $\kappa_{1}$ und der Übergangskonstanten $\rho$. Verwendet wurden 30 radiale Ansatzfunktionen und Startwerte $\rho_{\text {Start }}=0.9$ (statt $\rho=0.7$ ) und $\kappa_{1, \text { Start }}=0.8\left(\right.$ statt $\left.\kappa_{1}=1.0\right)$. Nach 60 Iterationen betrugen die Werte $\rho_{60}=0.699$ und $\kappa_{1,60}=0.999$ bei der Erdnuß und $\rho_{60}=0.727$ und $\kappa_{1,60}=1.053$ bei der Bohne; als Regularisierungsparameter wurde $\mu_{z}=.0005$ bei den Kurven und $\mu_{\rho}=\mu_{\kappa_{1}}=0.01$ bei den Konstanten gewählt. Die Gesamtlaufzeit betrug 9 Sekunden.
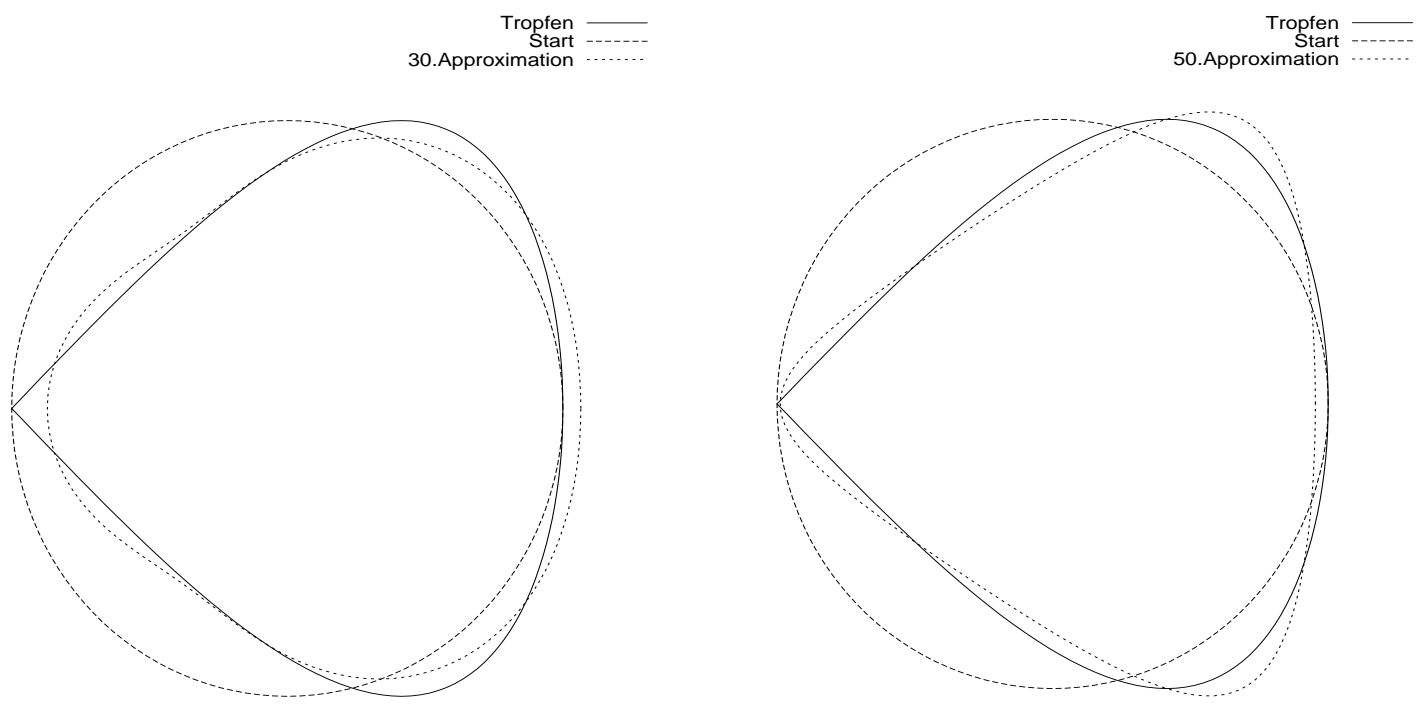

Abbildung 7.14: Rekonstruktion des Tropfen bei einer Einfallswelle; 64 Knotenpunkte auf der Approximationskurve. 10 Basisfunktionen; links sternförmig, rechts nichtradial; Laufzeit: 3 Sekunden. 

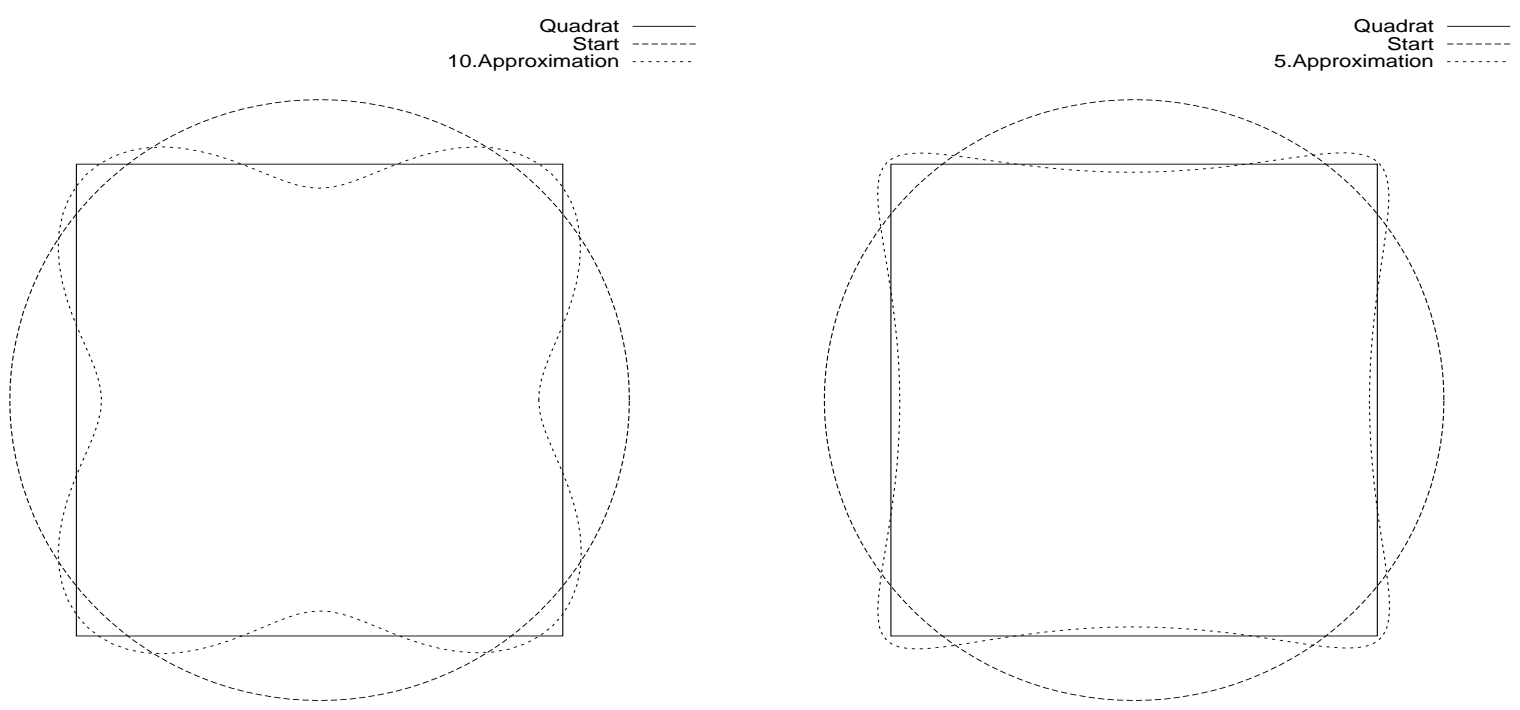

Abbildung 7.15: Rekonstruktion des Quadrats bei vier Einfallswelle; 64 Knotenpunkte auf der Approximationskurve. links 10 sternförmige Basisfunktionen, rechts 30 nichtradiale; Laufzeit: 1 Sekunden in beiden Fällen.

haben wir bei diesen Gebieten nur mit Einfachschichtpotentialen gearbeitet. In Abbildung 7.14 wurde der Tropfen, in Abbildung 7.15 das Quadrat betrachtet. In beiden Fällen liefert die nichtradiale Approximation bessere Rekonstruktionen. Das Newtonverfahren wird hier, insbesondere bei sternförmiger Approximation, schnell instabil.

Nun betrachten wir zwei Beispiele bei der Bohne, in denen wir fehlerhafte Daten verwendet haben. In Abbildung 7.16 (links) wurde mit fehlerhaften Konstanten rekonstruiert. In Abbildung 7.16 (rechts) bestand die Testgeometrie aus zwei Rändern (Bohne, die eine Erdnuß enthält). Bei der Rekonstruktion haben wir jedoch die Existenz der inneren Kurve vernachlässigt. Dieses zweite Beispiel liefert eine erstaunlich gute Rekonstruktion. Dies bedeutet, daß kleine Innengebiete wenig zu den Fernfelddaten beitragen. Dies hatten wir bereits beim direkten Problem gesehen, als die Konvergenz bei ineinanderliegende Gebiete erheblich langsamer war, als bei nebeneinanderliegenden Gebieten. Diese Beobachtung manifestiert sich in dem nächsten Versuch (vgl. Abbildung 7.17). Wir verwenden die gleiche Geometrie, d.h. eine Bohne, die eine Erdnuß enthält. Nun rekonstruieren wir beide Gebiete. Wir benötigen 16 Einfallswellen, um eine befriedigende Rekonstruktion zu erhalten. Man beachte den Vorteil der nichtradialen Approximation: bei der Erdnuß wurde neben der Form auch die Lage des Objekts rekonstruiert. In der Abbildung 7.18 wurden fünf nebeneinanderliegende Objekte rekonstruiert. Bereits bei der Verwendung von nur 16 Knotenpunkten auf den einzelnen Kurven und 32 Knotenpunkten bei der Norm 7.13 ist die Approximation befriedigend. Die Rechnung benötigte insgesamt nur 25 Sekunden.

Die Rekonstruktion einer komplizierteren Geometrie ist in Abbildung $7.19 \mathrm{zu}$ sehen. Wiederum zeigt sich, daß Strukturen im Innenraum eines anderen Gebietes erheb- 

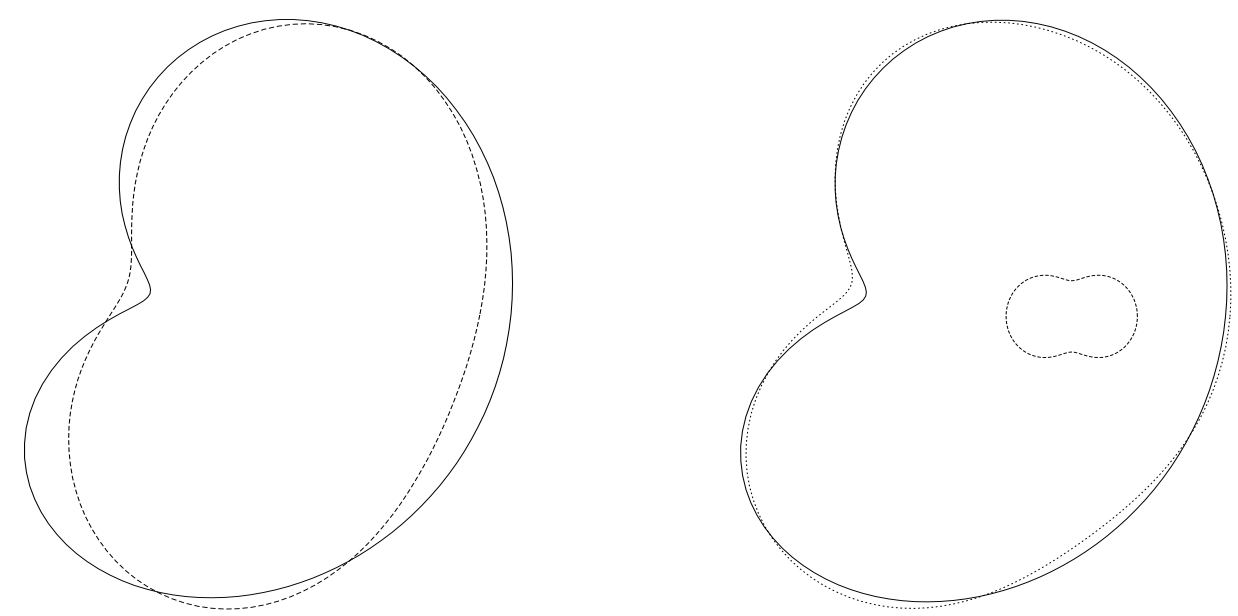

Abbildung 7.16: Links: Rekonstruktion der Bohne bei einer Einfallswelle und fehlerhaften inneren Konstanten, d.h. $\kappa_{i}=0.9$ statt 1.0 und $\rho=0.6$ statt 0.7. 5 Basisfunktionen; Laufzeit: $<1$ Sekunden bei 20 Iterationen; Rechts: Rekonstruktion der Bohne, die eine Erdnuß enthält; eine Einfallswelle und radiale Approximationskurve; bei der Rekonstruktion wird die innere Kurve nicht berücksichtigt. Laufzeit: 1 Sekunden bei 20 Iterationen.

lich schwerer zu rekonstruieren sind. Für eine befriedigende Rekonstruktion wurden 16 Einfallswellen benötigt. Der Regularisierungsparameter muß in dieser Situation relativ klein gewählt werden, da sonst das Verfahren zwar stabil ist, die inneren Strukturen aber kaum erkannt werden. Um trotzdem Stabilität zu gewährleisten, ist eine große Anzahl von Einfallswellen erforderlich.

\subsection{Vergleich mit anderen Resultaten und Fazit}

Ausgehend von den theoretischen Untersuchungen von Kirsch in [31] und Potthast in [48] wurden Newtonverfahren für das inverse Dirichlet- und Neumann-Problem bereits in einer Reihe von Arbeiten untersucht. Eine Literaturübersicht zu diesem Thema findet man in [7], Seite 132ff..

Im Gegensatz dazu wurden Rekonstruktionsverfahren für inverse Transmissionsprobleme weitaus seltener thematisiert. Erste Untersuchungen stammen von Vogel (vgl. [61]), der das Problem durch die Born-Approximation löste. Angell, Kleinman und Roach schlugen 1987 (vgl. [1]) ein Optimierungsverfahren zur Lösung des inversen Problems vor. Weitere Untersuchungen zu Verfahren dieser Art wurden von Colton und Monk (vgl. [8]) durchgeführt. Ebenfalls in die Gruppe dieser Verfahren, die die Lösung des direkten Problems umgehen, kann man eine von Kirsch und Kreß vorgeschlagene Methode (vgl. [7], Kapitel 5.4) einordnen. Dieses Verfahren wurde von Zinn in der Arbeit [66] zur Lösung des inversen Transmissionsproblems verwendet. Insbesondere berücksichtigte er auch Gebiete mit Ecken. 


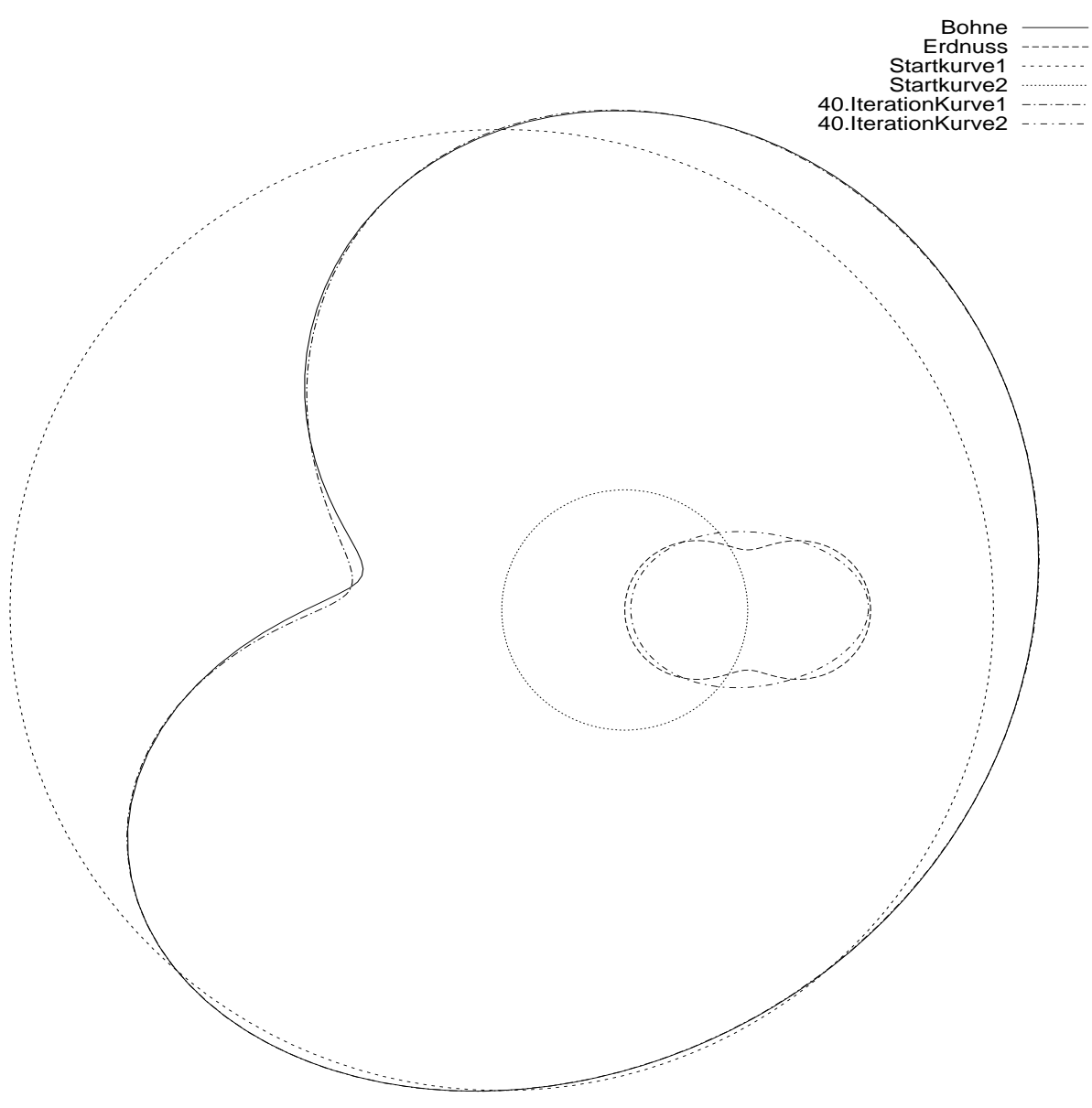

Abbildung 7.17: Rekonstruktion der Bohne, die eine Erdnuß enthält bei 16 Einfallswellen und zwei nichtradialen Approximationskurven. Laufzeit: 93 Sekunden bei 40 Iterationen 

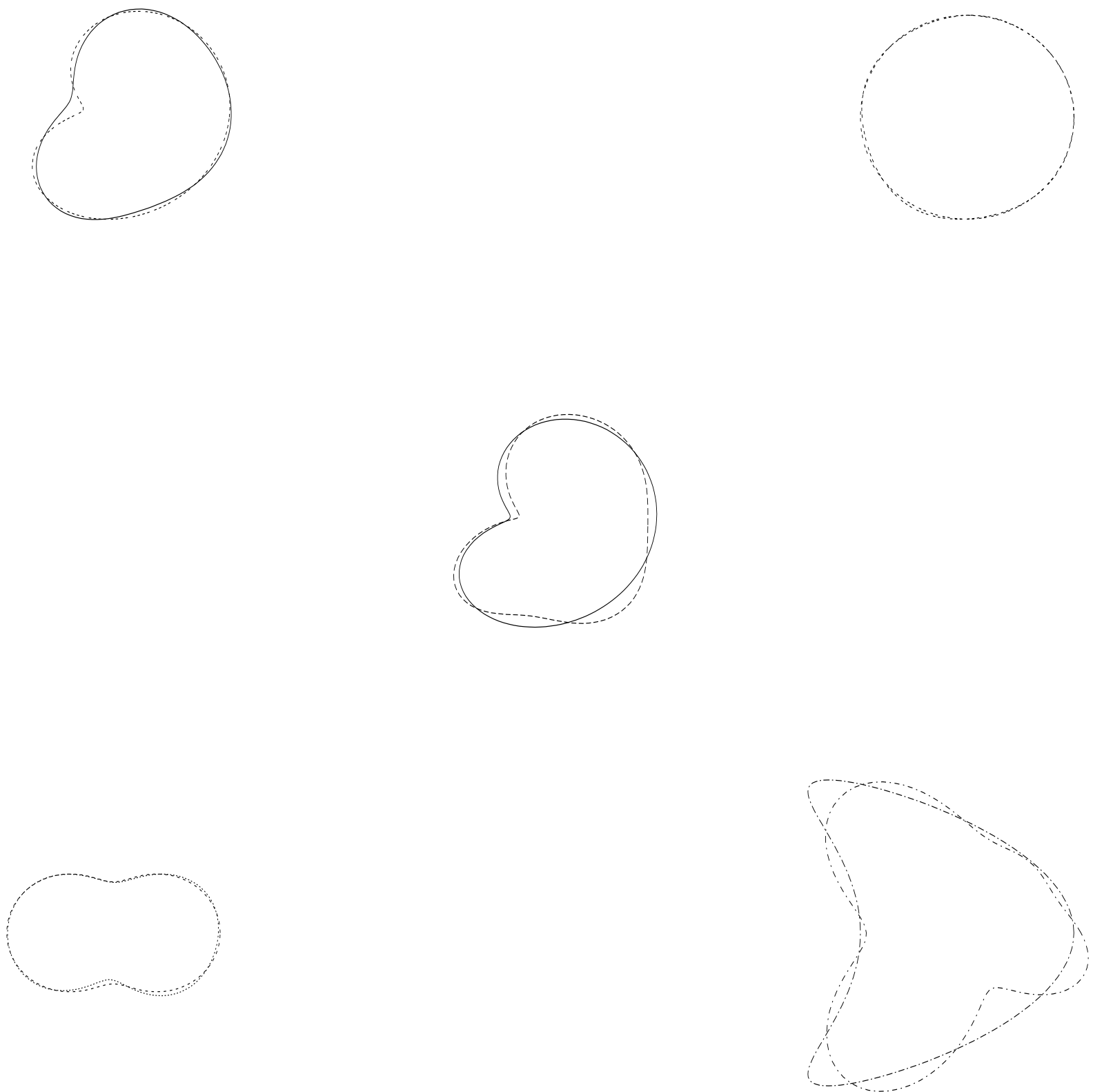

Abbildung 7.18: Rekonstruktion von fünf Gebieten bei acht Einfallswellen; 16 Knotenpunkte; 10 radiale Basisfunktionen; Laufzeit: 25 Sekunden bei 20 Iterationen 


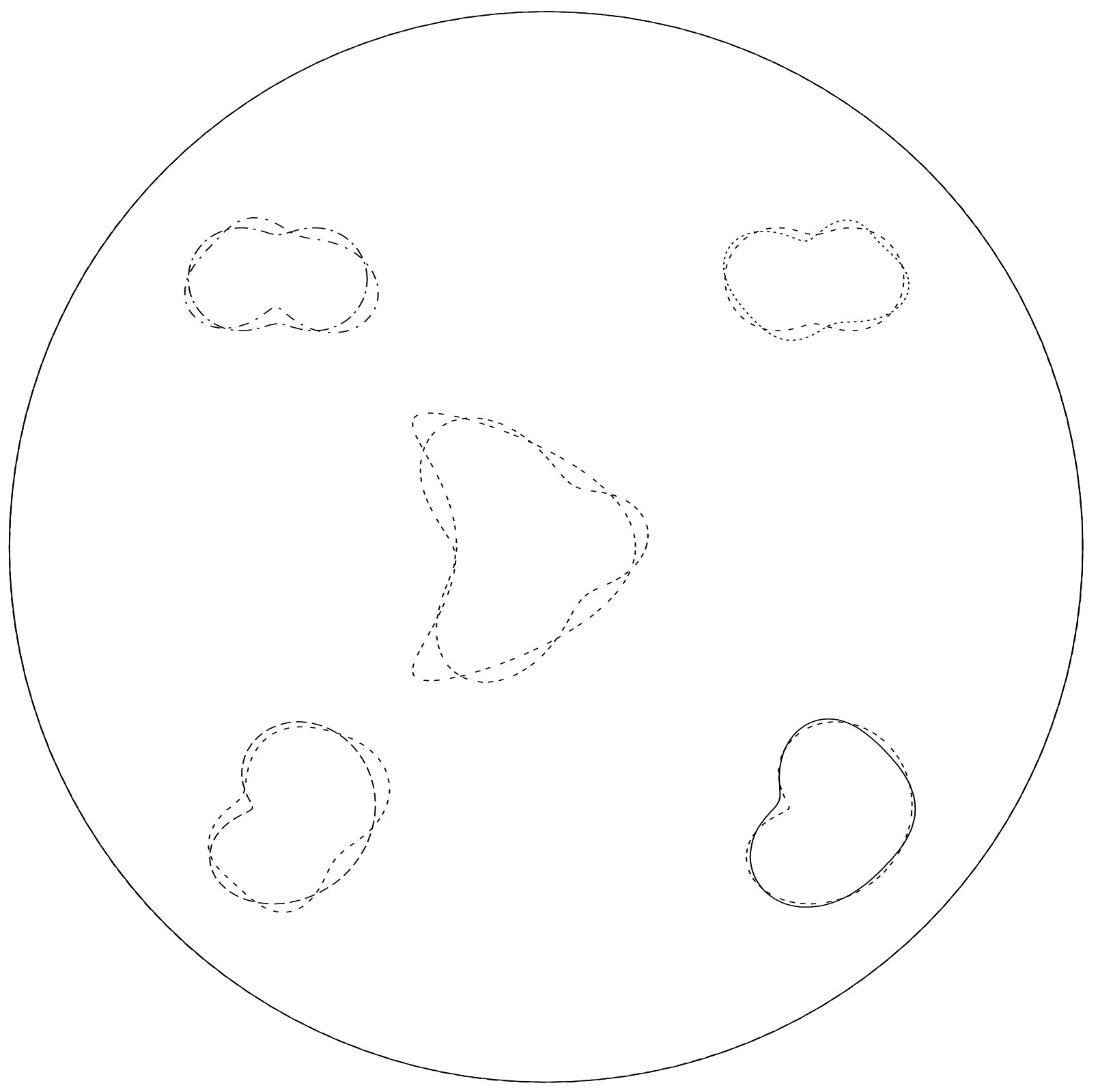

Abbildung 7.19: Rekonstruktion einer komplizierteren Geometrie; Wellenzahlen und Übergangskonstanten wie bei Geometrie 3 und zusätzlich $\kappa_{6}=0.8$ und $\rho_{6}=0.5$. 20 radiale Ansatzfunktionen pro Rand; Startwert des Regularisierungsparameters: 5e-5; 16 Einfallswellen; 32 Knotenpunkte auf den Kurven (64 zur Erhebung der Meßdaten); 64 Punkte zur Berechnung von $\|\cdot\|_{K}$ für die Ansatzfunktionen; 20 Iterationen; Zeitbedarf: 9 Minuten, 43 Sekunden 
Newtonverfahren zur Lösung des inversen Transmissionsproblems wurden in den Arbeiten [57] und [28] untersucht. Im Gegensatz zu dieser Arbeit wurde allerdings nur eine sternförmige Randkurve betrachtet. Im Vergleich zu den oben erwähnten Optimierungsverfahren sind die Rekonstruktionen beim Newtonverfahren im allgemeinen erheblich besser. Dies rechtfertigt den höheren Rechenaufwand, der durch das wiederholte Lösen von direkten Problemen entsteht. In der Arbeit [55] wird ebenfalls ein inverses Transmissionsproblem mit einem Newtonverfahren gelöst. Hier wird allerdings ein andere Vorgehensweise gewählt. Die Lösungen bzgl. eines gestörten Randes werden hier als Taylor-Reihe dargestellt. Die Taylorkoeffizienten sind gerade Ableitungen der Felder nach dem Rand, ausgewertet am ungestörten Rand, der bei den praktischen Rechnungen stets der Einheitskreis ist. Um den Konvergenzradius zu erhöhen wird zudem die Padé-Approximation verwendet. Im Gegensatz zu der hier gewählten Vorgehensweise können jedoch nur Objekte, die sich nicht zu stark von Kreisen unterscheiden, rekonstruiert werden. Dies schließt insbesondere nichtsternförmige Gebiete aus. Auch hier wird die innere Wellenzahl (bzw. das Verhältnis $\kappa_{0} / \kappa_{1}$ ) und der Übergangsparameter $\rho$ rekonstruiert, allerdings nicht durch Ausnutzung der Form der Ableitung, sondern durch Differenzenquotienten.

Nun wollen wir die Ergebnisse dieses Kapitels noch einmal kurz zusammenfassen. Zunächst haben wir die zur Lösung des direkten Transmissionsproblems in Kapitel 5 entwickelten Algorithmen numerisch getestet. Sowohl bei einem einzigen Rand, als auch bei mehreren Rändern, die nebeneinander, aber auch ineinander liegen konnten, konvergiert das Lösungsverfahren bei analytischen Rändern mit exponentieller Geschwindigkeit. Bei komplizierteren Geometrien sollte man iterative Verfahren zur Lösung des auftretenden Gleichungssystems verwenden.

Ferner haben wir Möglichkeiten zur Berechnung der benötigten Fréchet-Ableitungen angegeben. Die Ableitung nach dem Rand und der Übergangskonstanten $\rho$ kann durch ein direktes Transmissionsproblem bestimmt werden. Die Ableitungen nach inneren Wellenzahlen können durch Randwertprobleme zur inhomogenen Helmholtzgleichung charakterisiert werden. Um die numerische Lösung eines solchen Problems zu umgehen, haben wir einen alternativen Lösungsansatz verwendet.

Für die Verwendung eines regularisierten Newton-Verfahrens zur Rekonstruktion des Randes und der auftretenden Konstanten haben wir eine Reihe von Beispielen angegeben. Untersucht wurden sowohl sternförmige, als auch nichtsternförmige Gebiete. Neben der Form konnten wir auch die Lage eines Gebietes bestimmen. Weiterhin funktioniert das Verfahren auch für mehrere Ränder und es eröffnet die Möglichkeit, gleichzeitig die Ränder der Gebiete und die inneren Wellenzahlen und die Übergangskonstanten zu rekonstruieren. 


\section{Literaturverzeichnis}

[1] T. Angell, R. Kleinman, and G. Roach. An inverse transmission problem for the Helmholtz equation. Inverse Problems, 3:149-180, 1987.

[2] M. S. Berger. Nonlinearity And Functional Analysis. Academic Press, New York, San Francisco, London, 1977.

[3] O. P. Bruno and F. Reitich. Solution of a boundary value problem for the Helmholtz equation via variation of the boundary into the complex domain. Proc. $R$. Soc. Edinburgh, 122A:317-340, 1992.

[4] O. P. Bruno and F. Reitich. Calculation of Electromagnetic Scattering via Boundary Variations and Analytic Continuation. Appl. Com. Elec. Soc. Journal, 1:17-31, 1996.

[5] D. Colton and A. Kirsch. A simple method for solving inverse scattering problems in the resonance region. Inverse Problems, 12:383-393, 1996.

[6] D. Colton and R. Kress. Integral Equation Methods in Scattering Theory. Wiley, New York, 1983.

[7] D. Colton and R. Kress. Inverse Acoustic and Electromagnetic Scattering, Second Edition. Springer Verlag, Berlin, Heidelberg, New York, 1998.

[8] D. Colton and P. Monk. The inverse scattering problem for time harmonic acoustic waves in a penetrable medium. Quart. J. Mech. Appl. Math., 40:189-212, 1987.

[9] K. Deimling. Nonlinear Functional Analysis. Springer Verlag, Berlin, Heidelberg, New York, 1985.

[10] M. Deinlein. Die Gebietsableitung bei der Streuung an harten akustischen Körpern. Diplomarbeit, Erlangen, 1994.

[11] R. Djellouli, C. Farhat, J. Mandel, and P. Vanek. Continous fréchet differentiability with respect to a Lipschitz domain and a stability estimate for direct acoustic scattering problems. IMA Journal of Math. Analysis, 1998.

[12] O. Forster. Analysis I. Vieweg Verlag, Braunschweig, 1983.

[13] O. Forster. Analysis II. Vieweg Verlag, Braunschweig, 1984.

[14] O. Forster. Analysis III. Vieweg Verlag, Braunschweig, 1984. 
[15] K. Gerecke. Eine Integralgleichungsmethode zur Lösung von Transmissionsproblemen bei der Helmholtzgleichung. Diplomarbeit, Göttingen, 1990.

[16] D. Gilbarg and N. S. Trudinger. Elliptic Partial Differential Equations of Second Order, Second Edition. Springer Verlag, Berlin,Heidelberg, 1998.

[17] G. H. Golub and C. F. V. Loan. Matrix Computations, Third Edition. The John Hopkins University Press, London, 1996.

[18] A. Habbal. Nonsmooth shape optimization applied to linear acoustics. SIAM J. Optim., 8:989-1006, 1998.

[19] J. Hadamard. Lectures on Cauchy's Problem in Linear Partial Differential Equations. Yale University Press, New Haven, 1923.

[20] P. Hähner. On Acoustic, Electromagnetic, and Elastic Scattering Problems in Inhomogeneous Media. Habilitation, Göttingen, 1998.

[21] F. Hettlich. Frechet derivatives in inverse obstacle scattering. Inverse Problems, 11:371-382, 1995.

[22] H. Heuser. Lehrbuch der Analysis Teil 2. Teubner, Stuttgart, 1991.

[23] B. Hofmann. Mathematik inverser Probleme. Teubner, Stuttgart, Leibzig, 1999.

[24] T. Hohage. Newton-Verfahren beim inversen Neumann-Problem zur HelmholtzGleichung. Diplomarbeit, Göttingen, 1996.

[25] T. Hohage. Logarithmic convergence rates of the iteratively regularized GaußNewton method for an inverse potential and an inverse scattering problem. Inverse Problems, 13:1279-99, 1997.

[26] T. Hohage. Convergence rates of a regularized Newton method in sound-hard inverse scattering. SIAM J. Numer. Anal., 36:125-142, 1998.

[27] T. Hohage. Iterative Methods in Inverse Obstacle Scattering: Regularization Theory of Linear and Nonlinear Exponentially Ill-Posed Problems. Dissertation, Linz, 1999.

[28] T. Hohage and C. Schormann. A newton-type method for a transmission problem in inverse scattering. Inverse Problems, 14:1207-27, 1998.

[29] A. Kirsch. Generalized Boundary Value- and Control Problems for the Helmholtz Equation. Habilitation, Göttingen, 1984.

[30] A. Kirsch. Surface gradients and continuity properties for some integral operators in classical scattering theory. Math. Meth. Appl. Sciences, 11:789-804, 1989. 
[31] A. Kirsch. The domain derivative and two applications in inverse scattering theory. Inverse Problems, 9:81-96, 1993.

[32] A. Kirsch. An introduction to the mathematical theory of inverse problems. Springer Verlag, New York, Berlin, Heidelberg, 1996.

[33] A. Kirsch. Characterization of the shape of the scattering obstacle by the spectral data of the far field operator. Inverse Problems, 14:1489-1512, 1998.

[34] R. E. Kleinman and P. A. Martin. On single integral equations for the transmission problem of acoustics. SIAM J. Appl. Math., 48:307-325, 1988.

[35] M. Koecher. Lineare Algebra und analytische Geometrie. Springer Verlag, Berlin, Heidelberg, New York, Tokyo, 1985.

[36] R. Kress. On the numerical solution of a hypersingular integral equation in scattering theory. J. Comp. Appl. Math, 61:345 - 360, 1995.

[37] R. Kress. Linear Integral Equations, Second Edition. Springer Verlag, Berlin,Heidelberg, 1999.

[38] R. Kress. Acoustic scattering. Scattering, (Pike, Sabatier, eds.) a reference work by Academic Press, London, to appear.

[39] R. Kress and L. Päivärinta. On the far field in obstacle scattering. SIAM J. Appl. Math., 59:1413-1426, 1999.

[40] R. Kress and G. F. Roach. Transmission problems for the Helmholtz equation. J. Math. Phys., 19:1433-1437, 1978.

[41] R. Kress and I. H. Sloan. On the numerical solution of a logarithmic integral equation of the first kind for the Helmholtz equation. Numer. Math., 66:199-214, 1993.

[42] W. Kupradse. Randwertaufgaben der Schwingungstheorie und Integralgleichungen. VEB Deutscher Verlag der Wissenschaft, Berlin, 1956.

[43] R. Kussmaul. Ein numerisches Verfahren zur Lösung des Neumannschen Aussenraumproblems für die Helmholtzsche Schwingungsgleichung. Computing, 4:246273, 1969.

[44] R. Leis. Vorlesungen über partielle Differentialgleichungen zweiter Ordnung. Bibliographisches Institut AG, Speyer, 1967.

[45] E. Martensen. Potentialtheorie. Teubner Verlag, Stuttgart, 1968.

[46] A. Maue. Über die Formulierung eines allgemeinen Beugungsproblems durch eine Integralgleichung. Zeit. Physik, 126:601-618, 1949. 
[47] D. Mitrea. The transmission problem for multilayered anisotropic elastic bodies with rough interfaces. Numer. Funct. Anal. And Otimiz., 20:929-956, 1999.

[48] R. Potthast. Fréchet differentiability of boundary integral operators in inverse scattering. Inverse Problems, 10:431-447, 1994.

[49] R. Potthast. Fréchet-Differenzierbarkeit von Randintegraloperatoren und Randwertproblemen zur Helmholtzgleichung und zu den zeitharmonischen Maxwellgleichungen. PhD thesis, Göttingen, 1994.

[50] R. Potthast. A fast new method to solve inverse scattering problems. Inverse Problems, 12:731-742, 1996.

[51] R. Potthast. Fréchet differentiability of the solution to the acoustic Neumann scattering problem with respect to the domain. J.Inv.Ill-Posed Problems, 4:67-84, 1996.

[52] R. Potthast. A point-source method for inverse acoustic and electromagnetic obstacle scattering problems. IMA Journal of Appl. Math, 61:119-140, 1998.

[53] R. Potthast. Point-sources and multipoles in inverse scattering theory. Habilitation, Göttingen, 1999.

[54] D. N. G. Roy, L. Couchman, and J. Warner. Scattering and inverse scattering of sound-hard obstacles via shape deformation. Inverse Problems, 13:585-606, 1997.

[55] G. Roy, J. Warner, L. S. Couchman, and J. Shirron. Inverse obstacle transmission problem in acoustics. Inverse Problems, 14:903-929, 1998.

[56] A. F. Ruston. Fredholm Theory in Banach spaces. Cambridge University Press, Cambridge London New York, 1986.

[57] C. Schormann. Newton-Verfahren für ein inverses Transmissionsproblem bei elektromagnetischen Wellen. Diplomarbeit, Göttingen, 1996.

[58] J. Simon. Differentiation with respect to the domain in boundary value problems. Numer. Funct. Anal. and Optimiz., 2:649-687, 1980.

[59] S. P. und B. Silbermann. Projektionsverfahren und die näherungsweise Lösung singulärer Gleichungen. Teubner, Leibzig, 1977.

[60] S. P. und B. Silbermann. Numerical Analysis for Integral and Related Operator Equations. Akademie verlag, Birkhäuser, Berlin, Basel, 1991.

[61] C. Vogel. Numerical solution of a non-linear ill-posed problem arising in inverse scattering. Inverse Problems, 1:393-403, 1985. 
[62] K. Yosida. Functional Analysis. Springer Verlag, New York, Berlin, Heidelberg, 1995.

[63] E. Zeidler. Nonlinear functional analysis und its applications, volume 1. Springer Verlag, New York, Berlin, Heidelberg, 1993.

[64] E. Zeidler. Applied Functional Analysis, volume 2. Springer Verlag, New York, Berlin, Heidelberg, 1995.

[65] A. Zinn. Eine numerische Methode zur Lösung eines Transmissionsproblems bei der Helmholtzgleichung. Diplomarbeit, Göttingen, 1987.

[66] A. Zinn. Ein Rekonstruktionsverfahren für ein inverses Streuproblem bei der zeitharmonischen Wellengleichung. Dissertation, Göttingen, 1990. 



\section{LEBENSLAUF}

\section{Persönliche Daten}

$\begin{array}{ll}\text { Name } & \text { Christoph Schormann } \\ \text { Geburtstag } & 8.10 .1969 \\ \text { Geburtsort } & \text { Stuttgart } \\ \text { Staatsangehörigkeit } & \text { deutsch }\end{array}$

\section{SCHUlausbildung}

September 1976 - August 1980 Grundschule

September 1980 - August 1987 Gymnasium Casimirianum, Coburg

September 1987 - August 1988 Lauderdale County High School, Alabama, USA

September 1988 - Juni 1990 Gymnasium Casimirianum

29.Juni 1990

Abitur

\section{ZIVILDIENST}

September 1990 - Oktober 1991 Mauritiusschule Ahorn

\section{STUDIUM}

November 1991 - September 1993 Diplomstudium Mathematik an der Friedrich-

6. Mai 1993

Oktober 1993 - Januar 1997

17. Januar 1997 Alexander - Universität Erlangen-Nürnberg Vordiplom Mathematik Mathematikstudium an der GeorgAugust-Universität Göttingen

seit April 1997 Hauptdiplom Mathematik Promotionsstudium Mathematik gefördert durch die Deutsche Forschungsgemeinschaft

\section{Tätigkeiten am Fachbereich Mathematik DeR} Georg-August-Universitët Göttingen

Hilfswissenschaftler Fachgebiete numerische Mathematik, nichtlineare Funktionalanalysis, numerische Behandlung partieller Differentialgleichungen

Assistent Fachgebiete Funktionalanalysis, Optimierung, digitale Signalverarbeitung

Göttingen, den 15.Mai 2000,

Christoph Schormann 